\title{
Analytical and Experimental Study of the Acoustics and the Flow Field Characteristics of Cavitating Self-Resonating Water Jets
}

Georges L. Chahine, Philippe F. Genoux, Virgil E. Johnson, Jr., Gary S. Frederick Tracor Hydronautics 7210 Pindell School Rd Laurel, MD 20707

Prepared by Sandia Nationalusiofiratories Albuquerque, New Mexico 87185 and Livermore, California 94550 for the United States Department of Energy under Contract DE-AC04-76DP00789

Printed September 1984

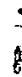

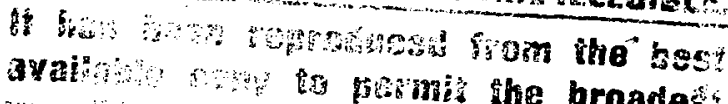

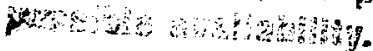


Issued by Sandia National Laboratories, operated for the United States Department of Energy by Sandia Corporation.

NOTICE: This report was prepared as an account of work sponsored by an agency of the United States Government. Neither the United States Government nor any agency thereof, nor any of their employees, nor any of their contractors, subcontractors, or their employees, makes any warranty, express or implied, or assumes any legal liability or responsibility for the accuracy, completeness, or usefulness of any information, apparatus, product, or process disclosed, or represents that its use would not infringe privately owned rights. Reference herein to any specific commercial product, process, or service by trade name, trademark, manufacturer, or otherwise, does not or service by trade name, trademark, manufacturer, or otherwise, does not by the United States Government, any agency thereof or any of their contractors or subcontractors. The views and opinions expressed herein do not necessarily state or reflect those of the United States Government, any agency thereof or any of their contractors or subcontractors.

Printed in the United States of America Available from

National Technical Information Service

U.S. Department of Commerce

5285 Port Royal Road

Springfield, VA 22161

NTIS price codes

Printed copy: A09

Microfiche copy: A01 


\section{DISCLAIMER}

Portions of this document may be illegible in electronic image products. Images are produced from the best available original document. 
Tracor Hydronautics

TABLE OF CONTENTS

Page

FOREWORD AND ACKNOWLEDGMENTS.................. ii

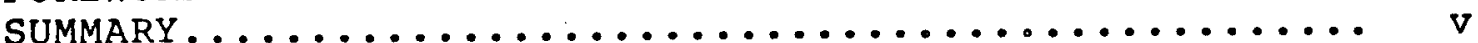

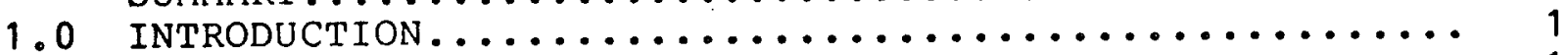

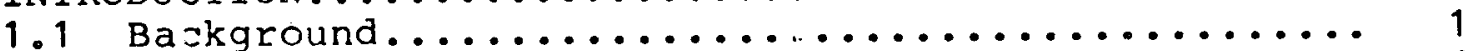

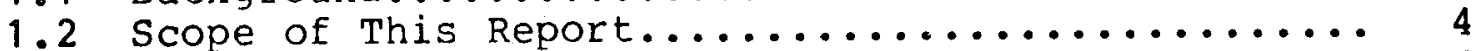

2.0 PIPE RESONANCE AND AMPLIFICATION................ 6

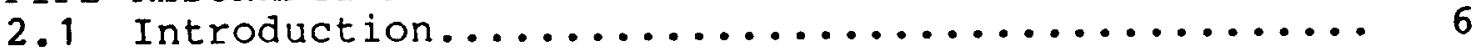

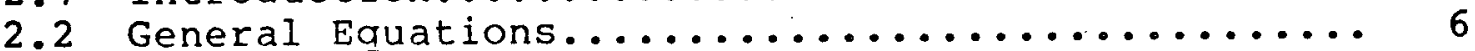

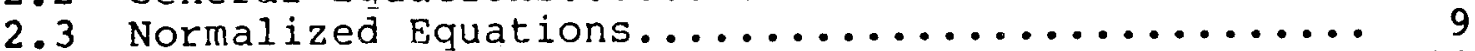

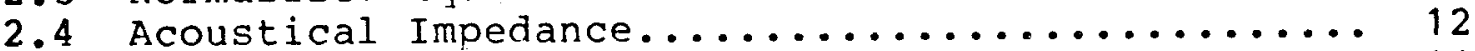

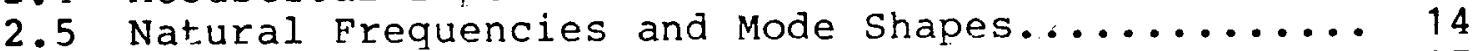

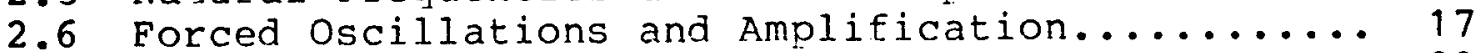

2.7 Numerical Examples and Conclusions............. 20

3.0 GENERAL FORMULATION OF VORTEX BUBBLE RING PROBLEM..... 24

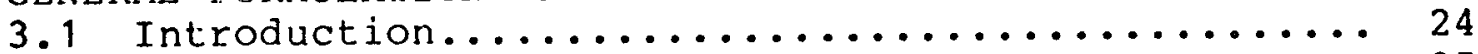

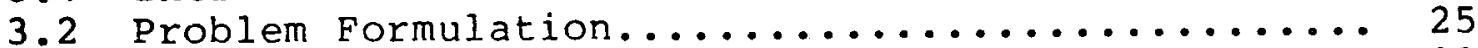

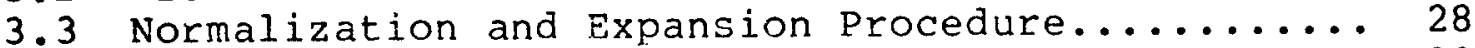

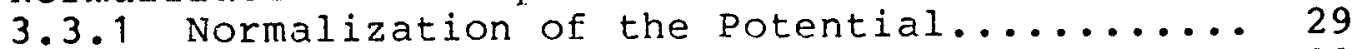

3.3.2 Normalization of the Pressures.............. 32

3.3.3 Normalization of the Translation Velocity. 32

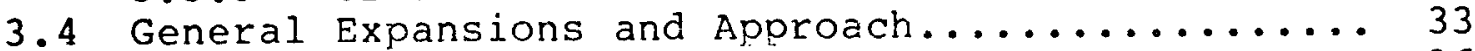

4.0 BUBBLE RING STATIC EQUILIBRIUM................. 36

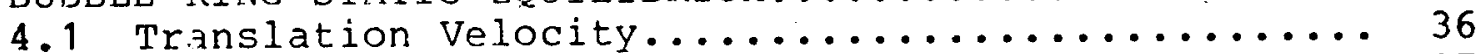

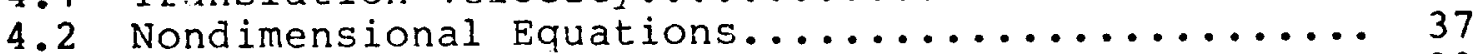

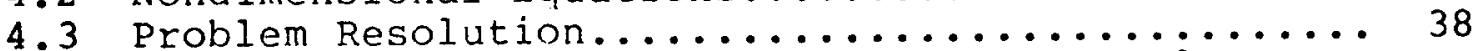

4.3.1 First Order Approximation, Order $\varepsilon^{\circ} \ldots . . .38$

4.3 .2 Order $\varepsilon \operatorname{Ln}(\varepsilon / 8)$ - Ring Motion........... 41

4.3 .3 Order $\varepsilon$ - Section Deformation............ 44

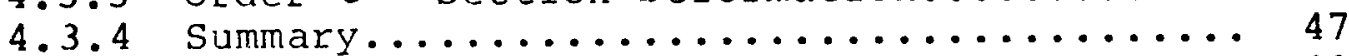

5.0 COLLAPSE OF A CAVITATING VORTEX RING............. 49

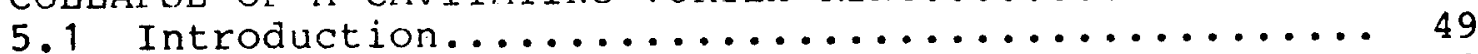

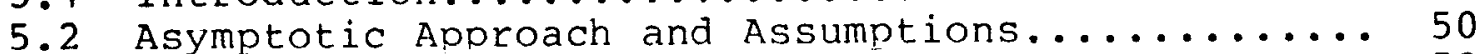

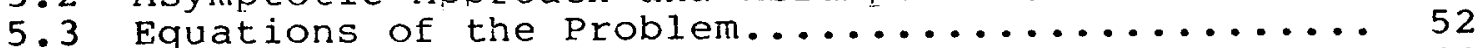

5.4 Characteristic Time of the Ring Dynamics....... 54

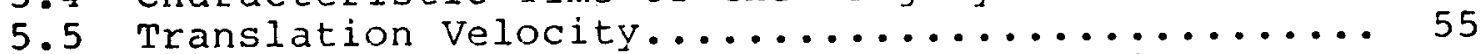

5.6 Problem Solution: Bubble Ring Oscillations

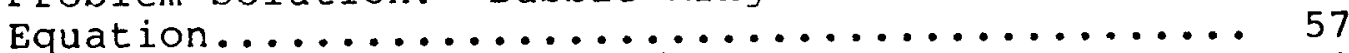

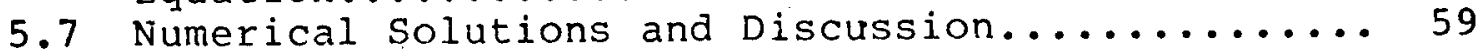

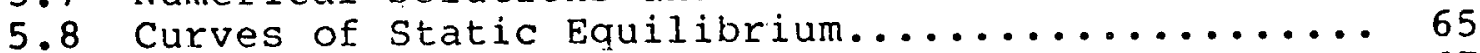

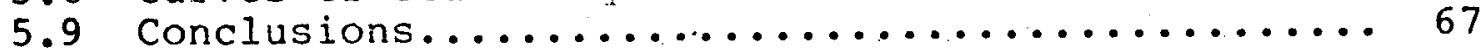

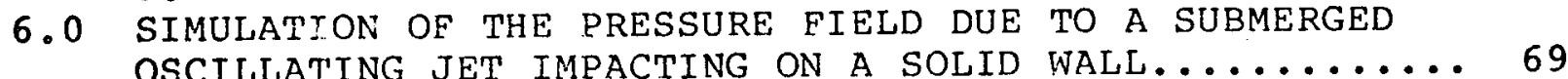

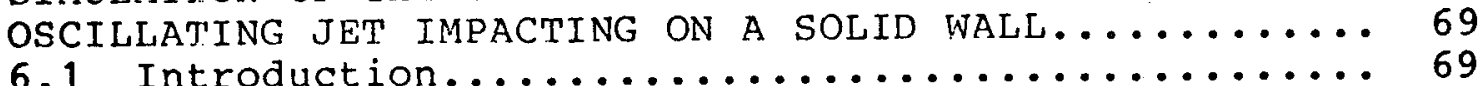

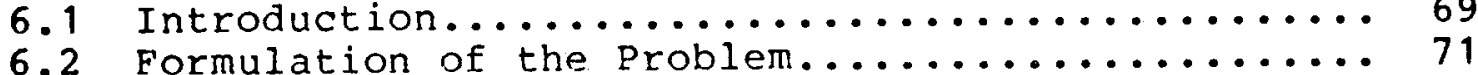

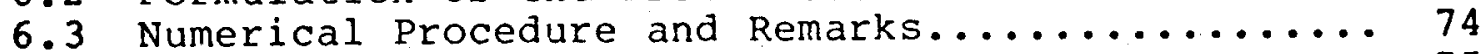

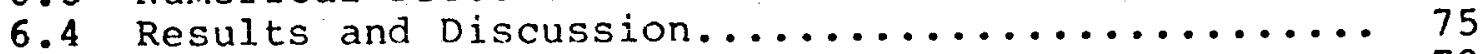

7.0 STUDY OF "LOW-SIGMA" OSCILLATING NOZZLES............ 78

\section{15
18
18}


TABLE OF CONTENTS (Continued)

7.1 Preliminary Analysis of Feedback Mechanism...... 79 7.2 Concepts for "Low-Sigma" Self-Resonating Nozzles.. 81 7.3 Experimental Results.................... 82

8.0 CONCLUSIONS AND RECOMMENDATIONS............... 86 REFERENCES

FIGURES

APPENDIX: Numerical Programs Listings 
Tracor Hydronautics

$$
\text { -iii- }
$$

FOREWORD AND ACKNOWLEDGMENTS

The study described in this report was conducted by riracor Hydronautics, Howard County, Laurel, Maryland. This program was supported by the U.S. Department of Energy/, Division of Geothermal Energy, under Sandia Laboratories Contract No. 68-8419.

The CAVIJET cavitating fluid jet technology used in this program has been patented by HYDRONAUTICS, Incorporated*, and additional patent applications related to the tuned-resonator STRATOJET have been submitted. The CAVIJET ${ }^{\otimes}$ technology is currently being developed for a variety of commercial applications.

The following organizations and individuals have made important contributions to the success of this phase of our investigations. The authors would like to express their sincere appreciation to:

- Sandia Laboratories - Dr. James R. Kelsey and Mr. David A. Glowka, for their support, advice, and encouragement throughout this program.

* U.S. Patent No. 3,528,704;3,713,699;3,807,632, and 4,262,757. Other U.S. and Foreign patents are pending or have been granted. CAVIJET is a registered trademark of HYDRONAUTICS, Incorporated.

\section{DISCLAIMER}

This report was prepared as an account of work sponsored by an agency of the United States Government. Neither the United States Government nor any agency thereof, nor any of their employees, makes any warranty, express or implied, or assumes any legal liability or responsibility for the accuracy, completeness, or usefulness of any information, apparatus, product, or process disclosed, or represents that its use would not infringe privately owned rights. Reference herein to any specific commercial product, process, or service by trade name, trademark, manufacturer, or otherwise does not necessarily constitute or imply its endorsement, recommendation, or favoring by the United States Government or any agency thereof. The views and opinions of authors expressed herein do not necessarily state or reflect those of the United States Government or any agency thereof. 
Tracor Hydronautics

$$
-i v-
$$

- Direction des Recherches Etures et Techniques, Paris, France which has supported the second author, Mr. Philippe Genoux, during his stay with us.

- Tracor Hydronautics, Inc. - Dr. Han Lieh Liu, Mr. william Lindenmuth, Mr. Charles Sirian, and Mr. George Matusky for contributions to various experimental and analytical tasks, and Dr. Andrew Conn for useful discussions. 


\section{SUMMARY}

Waterjet nozzles (STRATOJETS) have been developed which achieve passive structuring of cavitating submerged jets into discrete ring vortices, and which possess cavitation incipient numbers six times higher than obtained with conventional cavitating jet nozzles. In this study we developed analytical and numerical techniques and conducted experimental work to gain an understanding of the basic phenomena involved. The achievements are:

1. A thorough analysis of the acoustic dynamics of the feed pipe to the nozzle;

2. A theory for bubble ring growth and collapse;

3. A numerical model for jet simulation;

4. An experimental observation and analysis of candidate second-generation low-sigma STRATOJETS.

From this study we can conclude that intensification of bubble ring collapse and design of highly resonant feed tubes can lead to improved drilling rates. The models here described are excellent tools to analyze the various parameters needed for STRATOJET optimizations. Further analysis is needed to introduce such important factors as viscosity, nozzle-jet interaction, and ring-target interaction, and to develop the jet simulation model to describe the important fine details of the flow field at the nozzle exit. 
Tracor Hydronautics

-vi-

FOREWORD AND ACKNOWLEDGMENTS

The study described in this report was conducted by Traccr Hydronautics, Howard County, Laurel, Maryland. This program was supported by the U.S. Department of Energy, Division of Geothermal Energy, under Sandia Laboratories Contract No. 68-8419.

The CAVIJET ${ }^{\circledast}$ cavitating fluid jet technology used in this program has been patented by HYDRONAUTICS, Incorporated*, and additional patent applications related to the tuned-resonator STRATOJET have been submitted. The CAVIJET ${ }^{\otimes}$ technology is currently being developed for a variety of commercial applications.

The following organizations and individuals have made important contributions to the success of this phase of our investigations. The authors would like to express their sincere appreciation to:

- Sandia Laboratories - Dr. James R. Kelsey and Mr. David A. Glowka, for their support, advice, and encouragement throughout this program.

* U.S. Patent No. $3,528,704 ; 3,713,699 ; 3,807,632$, and 4,262,757. other U.S. and Foreign patents are pending or have been granted. CAVIJET is a registered trademark of HYDRONAUTICS, Incorporated. 
Tracor Hydronautics

\subsection{INTRODUCTION}

\subsection{Background}

The studies described in this report are part of an ongoing effort to understand and develop cavitating jets which will enhance the performance of bits used to drill for geothermal or fossil energy. During the first phase [1] we found that the existing CAVIJET ${ }^{\otimes}$ cavitating fluid jet nozzle designs were capable of operating under certain, limited downhole conditions, and improved rates of penetration (ROP) were observed with two-cone roller bits during both laboratory and field trials. Although these existing designs, now termed "conventional" or "basic" CAVIJET nozzles, gave encouraging results, the limits on their practical use motivated the study described in Reference [2].

Conventional CAVIJET nozzles were capable of producing cavitation, using drilling mud, to a depth of about $1,200 \mathrm{~m}$ $(4,000 \mathrm{ft})$ when operated at a nozzle pressure drop, $\Delta \mathrm{P}$, of about $13.8 \mathrm{MPa}(2,000 \mathrm{psi})$. However, due to the rather large size of these nozzles necessitated by their diameter contraction ratio, D/d, of four, the conventional CAVIJETS could only be fitted within a two-cone roller bit, a bit type which is seldom used in comparison to the widely accepted three-cone roller bit. Thus, the study summarized in

Reference [2] had the objective of discovering whether principles of "self-resonance" could be developed for improving the performance of submerged cavitating jets - thus eventually leading to new nozzle systems which are: (i) more erosive, (ii) capable of providing increased ROP at greater depths than conventional CAVIJET nozzles, and (iii) small enough to fit into the confines of existing three-cone roller bits with minimal modifications to the extended nozzle tubes now used in these drill bits. 
The results from this feasibility study demonstrated that self-resonating cavitating jet systems were indeed capable of providing the required performance for use in deep drilling three-cone bits. Specifically: (i) several passive resonating concepts were identified and tested; each provided structuring of the jet cavitation into well-defined ring vortices, (ii) the structured flows from these jets showed incipient cavitation numbers larger than conventional jets, thus providing cavitation erosion at greater depths, (iii) under comparable conditions, preliminary rock-cutting tests in water showed that a resonating jet was more erosive than a conventional cavitating jet, and (iv) preliminary versions of these new nozzle system concepts suggested that designs could be developed which would fit into three-cone bits, and within new bits which use PDC (polycrystalline diamond compact) cutting elements. These results and the recommendations developed during that phase of the program led to the effort described in Reference [3]. Some of the conclusions drawn from [3] are:

1. Several self-resonating nozzles systems*, the PULSER, PULSER-FED, and ORGAN-PIPE CAVIJET designs, produce passive oscillations which (a) cause the jet to structure into discrete ring vortices, and thus (b) provide nozzle systems which have incipient cavitation numbers two to six times higher than obtained with either conventional CAVIJET nozzles or typical drill bit nozzles.

2. In rock cutting trials, higher incipient cavitation numbers have been correlated with greater erosivity.

3. The ORGAN-PIPE CAVIJET system is adaptable to the physical restraints of existing bits, and is the most promising concept for future bit designs.

4. STRATOJET nozzle systems will clean chips from a simulated hole bottom at a nozzle pressure drop only about 25 percent of that required for standard nozzle systems.

An acronym: "STRATOJET" (STRuctured Acoustically Tuned Oscillating JET) will be used for these systems. 
Tracor Hydronautics

5. Self-resonating structured jets can be achieved either with or without cavitation. Therefore, at hole depths below where cavitation is present, a STRATOJET nozzle may still contribute to improved bottom hole cleaning.

It was also concluded in Reference [3] that, although nozzle designs which provide large amplitude pressure modulations were then available for operation in water, additional research was needed to optimize self-resonating jets for use:

(a) in mud,

(b) in specific drill bit designs,

(c) at higher system pressures than now currently used for deep-hole drilling.

Based on the conclusions reached in Reference [3], Sandia National Laboratories concluded that the technology for applying cavitating and structured jets to conventional mechanical bits operating at current nozzle pressure drops (less than 2500 psi) was sufficient for industry to carry out the necessary development to reach working hardware. Thus, industry has been encouraged to pursue the additional research recommended in [3]; that is, (a) in mud, and (b) in specific drill bit designs. On the other hand, sandia Laboratories did contract with Tracor Hydronautics to continue basic research on cavitating and structured jets as applied to higher system pressures than now currently used for deep-hole drilling.

This continued basic research on higher pressure cavitating and structured jets was conducted under sandia Contract No. 68.8419 , and the results of the investigation are the subject of the present report. 


\subsection{Scope of This Report}

The work described in the Background section definitely established that self-excited, structured jets have the potential to improve the drilling rate of conventional mechanical bits. This potential improved drilling rate is attributed to improved bottom cleaning, either by increased cavitation erosion or increased pressure fluctuation relief of bottom-hole chip hold-down caused by the passage of discrete vortices, or both. Increases in the jet velocity (nozzle pressure drop) will increase both these effects. It is expected that the vortex pressure reduction effect is linear with increase in nozzle pressure drop, $\Delta P$. However, all studies to date have indicated that cavitation erosion intensity increases as $\Delta \mathrm{P} n$, where $\mathrm{n}$ is approximately 3 . Thus, at sufficiently high pressures, a pure jet bit can be achieved.

Reference [4] reports that such pure jet bits have been built and tested using conventional nozzles and that very high drilling rates were achieved in hard formations at $\Delta \mathrm{P}$ greater than about 15,000 psi. The question which motivates the basic research conducted in the present research is: Can structured jets be developed which make it possible to achieve high drilling rates in a pure jet bit at pressures much less than 15,000 psi - say 7,500 psi?

In Reference [3] it was demonstrated that structured jets have an incipient cavitation number $\left(\sigma_{i}\right)$ of 2 or greater. Also, structured jets (at cavitation numbers of .1 to .3) were two to five times as erosive as conventional jets. If the value of $\sigma_{i}$ is 2 or greater, cavitation will occur at ambient pressures less than $2 \Delta \mathrm{P}$. If the value of $\Delta P$ is $7,500 \mathrm{psi}$, then cavitation will occur at depths less than 30,000 feet (or more if $\left.\sigma_{i}>2\right)$. Thus, already the cavitation effect will be possible for even the deepest hole if $\Delta P$ is 7,500 psi. If the 
erosion intensity varies as $(\Delta P)^{3}$ and conventional jets are adequately erosive to achieve an acceptable drilling rate for $\Delta \mathrm{P}-15,000$, the improvement in intensity attributed to replacing conventional jets with structured jets must be nearly an order of magnitude, if $\Delta \mathrm{P}$ is reduced from 15,000 to $7,500 \mathrm{psi}$. The maximum improvement reported in [3] is about 5 at a low cavitation number of 0.09 . This improvement was about 2.5 at a cavitation number of 0.4. No erosion data were obtained at cavitation numbers greater than about 0.4. Thus, the current structured jet technology indicates that a 7,500 psi pure jet bit is not yet possible. However, the structured jet technology is in its infancy. A better understanding of the detailed mechanics of such jets may well show the way to achieve the jump in performance required for the 7,500 psi pure jet bit. The research reported herein is a contribution toward gaining an improved understanding of the basic mechanics of structured jets.

The topics addressed in the present investigation are:

(1) A thorough analysis of the acoustic dynamics of the supply system to the nozzle;

(2) The general formulation of the vortex ring cavitation problem;

(3) A study of the static equilibrium of a ring vortex bubble;

(4) A theoretical analysis of an annular ring bubble collapse with imposed pressure rise;

(5) A theoretical development of the flow field associated with periodic emission of ring vortices;

(6) A discussion of the application of items (1) through (5) to the design of nozzles with $\Delta \mathrm{P}=4,000$ to 8,000 psi as applied to future bits;

(7) The results of observations and photographs of self-excited structured jets. 


\subsection{PIPE RESONANCE AND AMPLIFICATION}

\subsection{Introduction}

The understanding of the acoustics of the feed tube for an extended nozzle is fundamental for the design and operation of a self-resonating jet. An adequately designed sTRATOJET nozzle shape will provide, when operated in the proper velocity range, the desired feedback mechanism to the feed tube. This is achieved by a beneficial interaction between the jet shear layer and the nozzle lip. This phenomenon cannot be capitalized on without the presence of an acoustical resonator upstream of the nozzle which is excited through the feedback mechanism and which largely amplifies the oscillations. With the ORGAN-PIPE STRATOJET designs, this resonator is constituted of one or several successive, nearly constant section tubes. This is the case for the extended nozzle in a three-cone bit (Figure 1). The acoustical behavior of this tube or series of tubes (natural frequencies, modes, amplification factors...) determines whether the natural predominant frequencies of the submerged jet are amplified or not. Obviously, the optimum design objective is to feed back at the nozzle orifice these predominant natural frequencies of the jet to the resonant tubes and to match them with the oscillation frequencies of these feed tubes.

In our preceding technical report [3] we have initiated an acoustical analysis of the behavior of organ-pipe tubes. We will reformulate here a more general approach extending the study to include mode shapes as well as amplification factors. The effect of viscous losses will also be assessed.

\subsection{General Equations}

Let us consider the unidimensional flow of a liquid of density $\rho$, in a tube of cross-section area $s$. When the inertial 
Tracor Hydronautics

forces are much larger than the gravity forces, the equation of motion of the liquid in the tube can be written

$$
\frac{\partial v}{\partial t}+v \frac{d v}{d x}+\frac{1}{\rho} \frac{\partial p}{\partial x}+\lambda \frac{v\left|v^{n-1}\right|}{D^{m}}=0 \text {, }
$$

where $\mathrm{n}$ and $\mathrm{m}$ are experimental values. This last term accounts for the frictional losses in the tube and is written here in a generalized form as suggested in Reference [5]. The more classical expression is obtained when substituting $n=2$ and $m=1$. $\lambda$ is then the Darcy-Weisbach friction factor.

If we assume that the flow in the tube is mainly uniform, the velocity component normal to the flow direction will only be due to any changes in the cross-section area $s$. This could be due to either deformation of the tube, variation of its section in the flow direction, or to the presence of volume-changing cavities. In this case, the continuity equation can be written

$$
\frac{1}{S} \quad \frac{d S}{d t}+\frac{1}{\rho} \frac{d \rho}{d t}+\frac{\partial v}{\partial x}=0
$$

where $d / d t=\partial / \partial t+v \partial / \partial x$.

For a constant diameter rigid tube, this equation simplifies to the classical plane wave equation:

$$
\frac{1}{\rho c_{0}^{2}} \frac{d p}{d t}+\frac{\partial v}{\partial x}=0
$$

where $c_{0}$ is the sound speed in the liquid defined by

$$
c_{0}^{2}=\frac{\partial p}{\partial \rho}=\frac{K}{\rho}
$$

$\mathrm{K}$ is the bulk modulus of elasticity of the liquid. 
Tracor Hydronautics

When the tube elasticity is accounted for, $c_{0}$ is replaced by a modified sound speed $c$, such that

$$
c^{2}=\frac{R / \rho}{1+\frac{K}{E} \frac{D}{e} f(\mu)}
$$

where $E$ and $\mu$ are the Young's modulus and the Poisson ratio of the solid tube of diameter $D$ and wall thickness $e,[5] . f(\mu)$ is a function of the tube anchorage.

similarly, if the fluid in the tube contains bubbles, the modified expression depends on the gas volume or void fraction [6]. The expression of $c$ is then given by

$$
\frac{1}{c^{2}}=\frac{(1-\alpha)^{2}}{c_{0}^{2}}+\frac{\alpha^{2}}{c_{g}^{2}}+\frac{\rho \alpha(1-\alpha)}{P}
$$

where $c_{g}$ is the speed of sound in the gas. When the void fraction, $\alpha$, is neither close to 0 or $1, c$ can be approximated by:

$$
c^{2}=\frac{P}{\rho \alpha(1-\alpha)}
$$

One can notice that at atmospheric pressure, $c$ is as low as 300 $\mathrm{ft} / \mathrm{s}$ for a value of $\alpha$ of only one percent, while $\mathrm{c}_{0}$ is about $4,900 \mathrm{ft} / \mathrm{s}$.

Now, consider that the mean flow of characteristics $P, V$, has been perturbed and that $p^{\prime}$ and $V^{\prime}$ are respectively the pressure and velocity perturbations:

$$
\begin{aligned}
& p=p+p^{\prime} \\
& v=v+v^{\prime}
\end{aligned}
$$


Tracor Hydronautics

We will account for (2.8) in the basic equations, (2.1) and (2.3). If, in addition, the mean flow is steady and if we account for the fact that the mean velocity and pressure gradients for a constant section tube are negligible in the flow direction,

$$
\partial v / \partial x=\partial P / \partial x=0
$$

then the momentum and continuity equations become:

$$
\begin{aligned}
& \frac{\partial v^{\prime}}{\partial t}+v \frac{\partial v^{\prime}}{\partial x}+\frac{1}{\rho} \frac{\partial p^{\prime}}{\partial x}+f * v^{\prime}=0 \\
& \frac{\partial p^{\prime}}{\partial t}+v \frac{\partial p^{\prime}}{\partial x}+\rho c^{2} \frac{\partial v^{\prime}}{\partial x}=0
\end{aligned}
$$

$f *$ combines the actual value of $f$ as well as the considered powers of $D, V$, and other numerical factors.

\subsection{Normalized Equations}

In order to evaluate the relative importance of the different terms in equations (2.10) and (2.11), it is useful to choose the correct flow characteristics and to write the corresponding normalized equations. While there is no particular problem in the choice of the characteristic length, L, (namely the tube length for a single diameter pipe or a typical length for a multi-diameter pipe), the choice of the characteristic time is more critical. For the mean flow the characteristic velocity is the mean velocity $\mathrm{V}$ and as a result the characteristic time is $\mathrm{L} / \mathrm{V}$. However, for the acoustical velocity, $\mathrm{V}^{\prime}$, and pressure oscillations, $\mathrm{p}^{\prime}$, which are of interest here, the characateristic time should be $\mathrm{L} / \mathrm{c}$. In which case, if we normalize

$$
\begin{aligned}
& v^{\prime} \text { by } v_{0}{ }^{\prime}, \\
& p^{\prime} \text { by } p_{0}^{\prime}, \\
& t^{\prime} \text { by } L / c,
\end{aligned}
$$


Tracor Hydronautics

$\mathrm{x}$ by $\mathrm{L}$,

Equations (2.10) and (2.11) become:

$$
\begin{aligned}
& \frac{\partial \bar{v}^{\prime}}{\partial \bar{t}^{\prime}}+M \frac{\partial \bar{v}^{\prime}}{\partial \bar{x}}+\left(\frac{p_{O}^{\prime}}{\rho c v_{O}^{\prime}}\right) \frac{\partial \bar{p}^{\prime}}{\partial \bar{x}}+\left(\frac{f^{*} L}{c}\right) \bar{v}^{\prime}=0, \\
& \frac{\partial \bar{p}^{\prime}}{\partial \bar{t}}+M \frac{\partial \bar{p}^{\prime}}{\partial \bar{x}}+\left(\frac{\rho C v_{O}^{\prime}}{P_{O}^{\prime}}\right) \frac{\partial \bar{v}^{\prime}}{\partial x}=0,,
\end{aligned}
$$

where $M=V / c$ is the mean velocity Mach number. These normalized equations show that the most general description which conserves the maximum number of terms is such that

$$
\begin{aligned}
& p_{0}^{\prime}=\rho c v_{0}^{\prime}, \\
& M=O(1),
\end{aligned}
$$

and

$$
\overline{\mathrm{f}} *=\frac{\mathrm{f}^{\star} \mathrm{L}}{\mathrm{C}}=O(1)
$$

For the most common case of Darcy-Weisbach friction law, condition (2.16) becomes

$$
2 \mathrm{fM}=\mathrm{O}(\mathrm{D} / \mathrm{L})
$$

which shows that viscous effects are to be included when either the mean velocity Mach number, $M=V / c$, or the relative tube length, $\mathrm{L} / \mathrm{D}$, is large enough.

We will limit our study here to the case of low Mach number flows. This corresponds for a sound speed of $4,900 \mathrm{f} / \mathrm{s}$ to a pressure drop across the nozzle smaller than 162,000 psi! In this case, Equations $(2-12)$ and $(2-13)$ simplify at the first order in $M$ to: 
Tracor Hydronautics

$$
\frac{\partial \bar{p}^{\prime}}{\partial \bar{t}}+\frac{\partial \bar{p} \prime}{\partial \bar{x}}+\bar{f}^{*} \bar{v}^{\prime}=0
$$

$$
\frac{\partial \bar{p}^{\prime}}{\partial \bar{t}}+\frac{\partial \bar{v}^{\prime}}{\partial \bar{x}}=0
$$

and two decoupled equations for $\mathrm{p}^{\prime}$ and $\mathrm{V}^{\prime}$ can be derived:

$$
\begin{aligned}
& \frac{\partial^{2} \bar{v}^{\prime}}{\partial \bar{t}^{2}}+f^{*} \frac{\partial \bar{v}^{\prime}}{\partial \bar{t}}=\frac{\partial^{2} \bar{v}^{\prime}}{\partial \bar{x}^{2}} \\
& \frac{\partial^{2} \bar{p}^{\prime}}{\partial \bar{t}^{2}}+\overline{E^{*}}+\frac{\partial \bar{p}^{\prime}}{\partial \bar{t}}=\frac{\partial^{2} \bar{p}^{\prime}}{\partial \bar{x}^{2}}
\end{aligned}
$$

Simple solutions with separate variables can be obtained for this system of equations which can be written:

$$
\begin{aligned}
& \bar{p}^{\prime}(\bar{x}, \bar{t})=e^{\overline{s t}} \bar{p}^{\prime}(\bar{x}) \\
& \bar{v}^{\prime}(\bar{x}, \bar{t})=e^{\overline{s t}} \bar{v}^{\prime}(\bar{x})
\end{aligned}
$$

From here on we will use the complex notations for the variables. With this notation it is implied that the physical values of the velocity and pressure are the real parts of the complex number representing them. Therefore, in equations (2.22 and 2.23) $\mathrm{G}, \mathrm{P}^{\prime}$ and $\mathrm{V}^{\prime}$ are complex numbers. Solving for $\mathrm{P}^{\prime}$ and $V^{\prime}$ one obtains:

$$
\bar{p}^{\prime}(\bar{x}, \bar{t})=e^{\overline{s t}}\left(\bar{c}_{1} e^{\overline{\gamma x}}+c_{2} e^{-\overline{\gamma x}}\right)
$$


Tracor Hydronautics

$$
\bar{v} \cdot(\bar{x}, \bar{t})=-\frac{\bar{s}}{\bar{\gamma}} e^{\overline{s t}}\left(\bar{c}_{1} e^{\overline{\gamma x}}-\bar{c}_{2} e^{-\gamma x}\right)
$$

where

$$
\bar{r}^{2}=\bar{s}\left(\bar{s}+\bar{f}^{*}\right)
$$

$c_{1}$ and $c_{2}$ are complex constants which are determined by satisfying boundary conditions at the tube's ends.

\subsection{Acoustical Impedance}

To facilitate physical interpretation let us rewrite the last equations (2.24-2.26) in dimensional form. The actual pressures and velocity are the real parts of the expressions

$$
\begin{aligned}
& p^{\prime}(x, t)=e^{s t}\left(c_{1} e^{\gamma x}+c_{2} e^{-\gamma x}\right) \\
& v^{\prime}(x, t)=\frac{-s}{\rho \gamma c^{2}} e^{s t}\left(c_{1} e^{\gamma x}-c_{2} e^{-\gamma x}\right)
\end{aligned}
$$

where

$$
r^{2}=\left(s^{2}+f^{*} s\right) / c^{2}
$$

The complex number $s$ and $\gamma$ are respectively the wave frequency and the wave propagation constant.

We can now introduce the notion of acoustical impedance $[5,7]$. This is similar to the electrical approach, the analogy with the electrical problem being between acoustic pressure and electrical potential, flow rate, and current intensity. The complex acoustical impedance, $z$, is defined by

$$
z(x)=p^{\prime}(x, t) /\left[v^{\prime}(x, t) \cdot s\right]
$$


Tracor Hydronautics

$$
z(x)=|z(x)| e^{i \phi}
$$

where $|z(x)|$ is the impedance amplitude, and $\phi$ the phase lag between pressure and velocity at the location, $x$. The use of $z$ simplifies the analysis since, for instance, the two conditions at each end of the tube can be replaced by a single one.

For a pipe of constant section area, $s$, we can define a frequency-dependent pipe characteristic impedance:

$$
z_{c}=\gamma \rho c^{2} / s s
$$

$z_{c}$ is the acoustical impedance of an infinitely long pipe. Knowing for a given pipe (i), its characteristic impedance $z_{c i}$, as well as the end impedances, $z_{u i}$ at the pipe entrance $\left(x_{i}=0\right)$ and $z_{d i}$ at the pipe exit $\left(x_{i}=l_{i}\right)$, one can determine the constants $C_{1}$ and $C_{2}$ in equations $(2.27)$ and (2.28). From this the value of the acoustical impedance at any location $x_{i}$ can be determined and be written:

$$
z_{i}\left(x_{i}\right)=\frac{z_{d i}{ }^{-z_{c i}} \tanh \left(\gamma_{i} x_{i}\right)}{1-\left(z_{d i} / z_{c i}\right) \tanh \left(\gamma_{i} x_{i}\right)}
$$

From this general expression we can relate upstream to downstream values of the acoustical impedance as follows:

$$
\begin{aligned}
& z_{u i}=\frac{z_{d i}+z_{c i} \tanh \left(\gamma_{i}^{\ell_{i}}\right)}{1+\left(z_{d i} / z_{c i}\right) \tanh \left(\gamma_{i} \ell_{i}\right)} \\
& z_{d i}=\frac{z_{u i}-z_{c i} \tanh \left(\gamma_{i}^{\ell}{ }_{i}\right)}{1-\left(z_{u i} / z_{c i}\right) \tanh \left(\gamma_{i} l_{i}\right)}
\end{aligned}
$$




\subsection{Natural Frequencies and Mode Shapes}

Let us consider the general case of a series of pipes having different cross sections. Each of the individual pipes has a constant section and can be treated acoustically as a single pipe with given end impedances. Equations (2.33) and (2.34) give the relations between these end impedances. To these equations we have to add the matching conditions of the pressures and flow rates at the connecting station of two successive pipes. This can be written using the impedances:

$$
z_{u i}=z_{u i-1}
$$

If, in addition, we know the values of the impedances at the two ends of the multipipe system, we have a complete set of equations to determine the wave frequency, s. This frequency being the same for all pipe sections, the various wave propagation constants, $\gamma_{i}$, are given by the relation (2.29) written as follows:

$$
r_{i}^{2}=\left(s^{2}+f_{i}^{*} s\right) / c^{2}
$$

The value of the end impedance, $z_{e}$, which is also the downstream impedance of the last tube, $z_{d n}$, is a known expression which has been determined experimentally for different types of end conditions $[5,7]$. Figure 2, taken from our preceding Sandia report [3] summarizes the most common end conditions.

We will now consider as an illustration the case of a three-pipe system, (Figure 3). This corresponds to a schematization of the three-cone bit tube (see Figure 1). Then with

$$
H_{i}=\tanh (\gamma \ell)_{i}
$$


we have for the impedances at the ends of each tube (see Figure 3):

$$
\begin{aligned}
& z\left(l_{1}+l_{2}+l_{3}\right)=z_{e}
\end{aligned}
$$

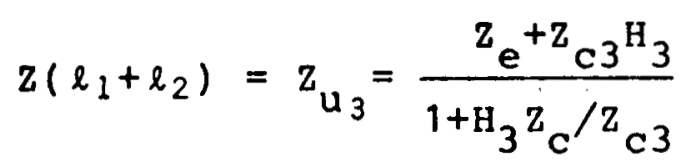

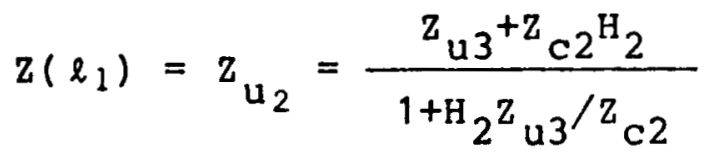

$$
\begin{aligned}
& z(0)=z_{u_{1}}=\frac{z_{u 2}+z_{c 1} H_{1}}{1+H_{1} z_{u 2} / Z_{c 1}}
\end{aligned}
$$

By combining (2.39), (2.40), and (2.41) one obtains a single relation tetween $z_{u l}$ and $z_{e}$. If the pipe system is connected upstream to a plenum where the pressure remains constant, then $z_{u l}=0$, and the characteristic equation to solve for $s$ simplifies to:

$$
\left(\mathrm{Z}_{\mathrm{e}}+\mathrm{Z}_{\mathrm{c} 3} \mathrm{H}_{3}\right)+\left(1+\frac{\mathrm{Z}_{\mathrm{e}}}{\mathrm{Z}_{\mathrm{c} 3}} \mathrm{H}_{3}\right)\left(\mathrm{Z}_{\mathrm{c} 2} \mathrm{H}_{2}+\mathrm{Z}_{\mathrm{c} 1} \mathrm{H}_{1}\right)+\left(\frac{\mathrm{Z}_{\mathrm{e}}}{\mathrm{Z}_{\mathrm{c} 2}}+\frac{\mathrm{Z}_{\mathrm{c} 3}}{\mathrm{Z}_{\mathrm{c} 2}} \mathrm{H}_{3}\right) \mathrm{Z}_{\mathrm{c}_{1}} \mathrm{H}_{1} \mathrm{H}_{2}=0
$$

The left hand side of (2.42) is a complex number $C$ of the complex variable $s$, and the resolution of the problem consists in looking for the zeros of $c$ :

$$
C(s)=C(\sigma+i \omega)=0
$$

To do so we chose the following method. We consider the modulus of $C,|C|$, and look for its minimums when $\sigma=0$. (starting by fixing $\sigma$ to zero is not a necessity but reduces computation time since we know that the attenuation factor, $\sigma$, is small.) Then, departing from each of these minimums, we search for the location 
Tracor Hydronautics

of the zeros of $\mathrm{C}$ by moving along the lines of steepest descent on the surface $|c|(\sigma, \omega)$ (Figure 4a), or along the tangent of the complex curve $\mathrm{C}(\mathrm{S})$ (Figure $4 \mathrm{~b}$ ) using Newton's method. At each computation step, the slope of the function $C(s)$ is determined, and the new value of $s$ is obtained by intersection of the tangent at the considered point, $s_{1}$, of the s-axis. This is obtained by writing

$$
\Delta s_{1}=-C\left(s_{1}\right) / C^{\prime}\left(s_{1}\right)
$$

The derivative $C^{\prime}\left(s_{1}\right)$ is obtained numerically by considering a very small step, $\varepsilon$. Thus, $\Delta s_{1}$ is in practice obtained by the following numerical expression:

$$
\Delta s_{1}=-\varepsilon C\left(s_{1}\right) /\left[C\left(s_{1}+\varepsilon\right)-C\left(s_{1}\right)\right]
$$

Once the values of $s$ solution of (2.43) are found, the

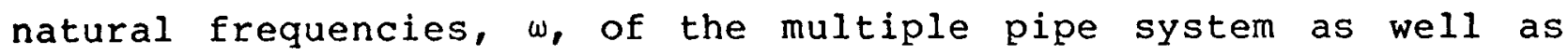
the attenuation factor, $\sigma$, are found. We should notice that a negative value of $\sigma$ means attenuation with time of the oscillation, while a positive value will indicate instability and amplification with time. We will present below (paragraph 2.7) some results obtained for particular multi-pipe configurations corresponding to the three-cone bit.

The acoustical pressures $P^{\prime}(x)$ and the flow rates, $Q^{\prime}(x)=S V^{\prime}(x)$, are determined for a three-pipe tube using the following expressions:

$$
\begin{aligned}
& P_{3}{ }^{\prime}(x)=P_{e}^{\prime} \cosh \gamma_{3}\left(l_{t}-x\right)+z_{c_{3}} Q_{e}^{\prime} \sinh \gamma_{3}\left(l_{t}-x\right), \\
& Q_{3}{ }^{\prime}(x)=\frac{P_{e}^{\prime}}{z_{c_{3}}} \sinh \gamma_{3}\left(l_{t}-x\right)+Q_{e}^{\prime} \cosh \gamma_{3}\left(l_{t}-x\right) ;
\end{aligned}
$$


Tracor Hydronautics

$$
\begin{aligned}
& P_{2}{ }^{\prime}(x)=P_{3}{ }^{\prime}\left(l_{a}\right) \cosh \gamma_{2}\left(\ell_{a}-x\right)+z_{c_{2}} Q^{\prime}\left(\ell_{a}\right) \sinh \gamma_{2}\left(\ell_{a}-x\right), \\
& Q_{2}{ }^{\prime}(x)=\frac{P_{3}{ }^{\prime}\left(l_{a}\right)}{z_{c_{2}}} \sinh \gamma_{2}\left(l_{a}-x\right)+Q^{\prime}\left(l_{a}\right) \cosh \gamma_{2}\left(l_{a}-x\right) \text {; } \\
& P_{1}^{\prime}(x)=P_{2}{ }^{\prime}\left(\ell_{1}\right) \cosh \gamma_{1}\left(\ell_{1}-x\right)+Z_{c_{1}} Q^{\prime}\left(\ell_{1}\right) \sinh \gamma_{1}\left(\ell_{1}-x\right), \\
& Q_{1}^{\prime}(x)=\frac{P_{2}{ }^{\prime}\left(\ell_{1}\right)}{Z_{c_{1}}} \sinh \gamma_{1}\left(\ell_{1}-x\right)+Q^{\prime}\left(\ell_{1}\right) \cosh \gamma_{1}\left(\ell_{1}-x\right) \text {. }
\end{aligned}
$$

In these expressions $\mathrm{P}_{\mathrm{e}}{ }^{\prime}$ and $Q_{e}{ }^{\prime}$ are the exit end acoustical pressure and flow rate, and $\ell_{t}, \ell_{a}$ are defined by:

$$
\begin{aligned}
& \ell_{t}=\ell_{1}+\ell_{2}+\ell_{3} \\
& \ell_{a}=\ell_{1}+\ell_{2}
\end{aligned}
$$

Again, paragraph 2.7 will present the mode shapes and the phase lags (see Eq. $(2.30)$ ) corresponding to the extended tube of the three-cone bit.

\subsection{Forced Oscillations and Amplification}

We have seen earlier that for a single tube, acoustical pressure and velocity satisfy the two decoupled equations (2.20) and (2.21). Separating the variables (time, space) as follows:

$$
\begin{aligned}
& p^{\prime}(x, t)=\pi(t) \cdot P^{\prime}(x) \\
& v^{\prime}(x, t)=U(t) \cdot V^{\prime}(x)
\end{aligned}
$$

one finds in dimensional form the following equations:

$$
\begin{aligned}
& P^{\prime} x x-r^{2} P^{\prime}=0 \\
& V^{\prime} x x-r^{2} V^{\prime}=0
\end{aligned}
$$




$$
\begin{aligned}
& \pi_{t t}+f \star \pi_{t}-c^{2} \gamma^{2} \pi=0 \\
& U_{t t}+f * U_{t}-c^{2} \gamma^{2} U=0
\end{aligned}
$$

where subscripts $\mathrm{x}$ or $t$ indicate derivation with respect to $\mathrm{x}$ or $t$. Equations (2.51), (2.52) show that the system acts as a harmonic oscillator both space- and time-wise.

Let us consider now the forced oscillations of such a system by concentrating on the equation for $U$ which is the easiest to interpret. All terms in this equation are variations with time of forces per unit mass of the liquid. Let us assume now that the oscillations of $U$ are forced by an external oscillating rate of force per unit mass of value $A e^{i w t}$. The equation of the oscillations of the forced system becomes:

$$
U_{t t}+f * U_{t}-c^{2} \gamma^{2} U=A c^{i \omega t}
$$

We will consider the interesting case where $\omega$ is the natural frequency of the system. The complex number $\gamma$ is related to $s$, solution of (2.43), and to $\omega$ through the relation (2.29) and $s=\sigma+i \omega$. If the inertia and friction terms of the system (time derivative terms) were negligible, the amplitude of the oscillations would be

$$
B_{0}=-A / C^{2} \gamma^{2}
$$

However, the oscillator responds to the excitation and the solution is of the type

$$
U=a e^{s t}+U_{1}
$$

where the first term is the natural solution of the free system and $U_{1}$ is a particular solution of the forced oscillator. The first term dies out with time due to damping $(\sigma<0)$. A particular solution $U_{1}$ of the form, 
Tracor Hydronautics

$$
U_{1}=B_{1} e^{i \omega t}
$$

is found provided that

$$
B_{1}=-A /\left(\sigma^{2}+2 i \sigma \omega+f^{*} \sigma\right)
$$

The amplification of the system is therefore defined as

$$
A_{m p}=\frac{B_{1}}{B_{0}}=\frac{s^{2}+f^{\star} s}{\sigma^{2}+2 i \sigma \omega+f^{*} \sigma}
$$

with $s=\sigma+i \omega$. An approximation of equation (2.58) can be obtained when $\mathrm{f}^{*}$ is negligible (frictionless system):

$$
A_{m p}=\frac{\sigma^{3}+3 \sigma \omega^{2}-2 i \omega^{3}}{\sigma\left(\sigma^{2}+4 \omega^{2}\right)}
$$

The absolute value of the complex amplification, $A_{m p}^{\circ}$ is then

$$
\left|A_{m p}^{\circ}\right|=\frac{4 \mu^{6}+9 \mu^{4}+6 \mu^{2}+11}{4 \mu^{2}+1}
$$

where,

$$
\mu=\omega / \sigma
$$

。

For very large values of $\mu$ we can approximate $\left|A_{m p}\right|$ with:

$$
\left|A_{m p}^{\circ}\right| \simeq \frac{|\mu|}{2}=\frac{-\omega}{2 \sigma}
$$


The analysis presented above gives only an idea of the phenomenon and lacks any detailed analysis or the forcing system. The wave equation (2.52) considered at the exit of the multipipe system should provide the pressure or velocity fluctuations at this end. However, it is not clear what is the physical forcing function. We know from experience that it is due to the reaction of the exiting jet downstream, and thus from the turbulence organization, but additional investigation and analysis is needed to determine if the connecting forcing function factor between the resonating system (pipes) and the jet is a rate of force per unit mass as presumed above.

\subsection{Numerical Examples and Conclusions}

In this subsection, several numerical examples of the method described above are presented. For all the cases here presented the overall length of the two- or three-stepped tubes is 8 inches, and the exit nozzle diameter is 0.3 inches. This corresponds to the straightened extended nozzle feed-tube of a three-cone roller bit presented in Figure 1. Five difrerent configurations of two successive cylindrical tubes of 0.80 inch and 0.65 inch diameter are considered. A sixth configuration corresponds to three tubes of equal lengths ( $22 / 3$ inches) and with $1,0.8$, and 0.65 inch diameters. For all these cases, the various values of the attenuation factor $\sigma$, and natural frequencies, $\omega$, were computed using the method described earlier. The results are summarized in Figures 5 and 6 for $M=0.05$ and 0.1 , which correspond to the functioning points, $\Delta \mathrm{P} \simeq 400$ and $1600 \mathrm{psi}$, respectively. The attenuation factor is seen to increase with the Mach number since viscous damping increases with velocity. Figures 7 and 8 show, for the same cases, the ratios of wavelengths to total tube length $\lambda / l_{r_{1}}$, and the amplification gain, $\beta_{0}$, defined as the ratio of the stepped tube gain to the straight tube gain. 
A comparison between Figures 5 to 8 show that $B_{0}$ increases when the attenuation factor a decreases. Another interesting observation is the presence of certain modes at a given Mach number and the absence of them at another Mach number. This experimentally observed phenomena is illustrated for the sixth configuration, $\ell_{1}=\ell_{2}=\ell_{3}$, where only the third and sixth mode appear at $M=0.1$ while all six first modes are present at $M=0.05$. It is also interesting to note that the higher $B_{0}^{\prime} s$ observed in Figures 7 and 8 are precisely for those modes disappearing at the higher Mach number. Similarly, the corresponding attenuation factors are the lowest. This will be discussed later.

Figures 9 and 10 summarize the results presented in the tables of Figures 5 to 8 . One can see that while the amplification of a straight pipe varies linearly from one mode to another, that of a two- or three-stepped tube oscillates about that linear behavior. Figure 9 shows for a Mach number of 0.05 that the most amplified configurations are the three-stepped tubes of equal lengths $\left(\ell_{1}=\ell_{2}=\ell_{3}\right)$ and the $3.2-4.8$ inch configuration. This latter was shown experimentally to give the highest pressure fluctuations. On the same figure the range of practical interest $0.3<\mathrm{s}_{\mathrm{d}}<0.7$ is shown; and for the main frequency, $\mathrm{f} \approx 3675 \mathrm{~Hz}$, the above-described configuration shows gains of 29 and 22 percent, respectively. At the higher Mach number, $M=0.1$ (Figure 10), the gain is less important in the useful range, and the 2.6-5.4 inch configurations seem to perform best. Figure 11 shows for that particular configuration how the amplification gain varies with the strouhal number. It is important to notice that the three values of the Mach number considered seem to lie on the same oscillatory curves which peak around $s_{d} \simeq 0.5$ in the interesting zone. Obviously, more is to be learned about how such a curve changes from one configuration to another. 
As an illustration of the study presented in section 2.5 on mode shapes, and in order to interpret some of the results shown above, we consider the case of a three-stepped tube, $\ell_{1}=\ell_{2}=\ell_{3}$. Figures 12 to 14 show (up to the sixth mode) how the normalized fluctuating flow rates, pressures, and impedances vary along the tube length. As said earlier, when the flow velocity is such that the Mach number is 0.1 , only the third and the sixth mode appear as solution of the equations (if we limit ourselves to the sixth mode). Figure 12 shows for these two modes the mode shapes. We observe that the third mode for the total tube is the first mode for each pipe of constant diameter which constitutes it. As imposed in the problem, the impedance is zero upstream and given by $z_{e}$ downstream. $z_{e}$ has been calculated in the preceding report [3] to be

$$
z_{e}=z_{c_{3}} M\left(s_{3} / s_{j}-s_{j} / s_{3}\right)
$$

where $\mathrm{Z}_{\mathrm{C}_{3}}$ is the characteristic impedance of the last pipe section as defined by (2.31). $S_{j}$ is the jet section area, $s_{3}$ the pipe section area, and $M$ the Mach number.

The pressure fluctuation is imposed to be five percent of the pressure drop acros the nozzle and zero upstream. Figure 12 shows the corresponding pressure oscillations in the tube. similarly, we can observe the flow rate curves. At the tube exit, the flow rate fluctuations are imposed once $z_{e}$ and $p$ are known. We can observe for both the pressure and the flow rate, incremental amplification at each pipe section.

Figures 13 and 14 show again the mode shapes for the same tube and the same imposed normalized pressure fluctuations at the exit but for a smaller velocity, $M=0.05$. Here all six. first modes are solutions, and as observed in Figure 7, the highest amplifications are obtained for the first, second, fourth, fifth modes. Figures 13 and 14 might provide an explanation for 
Tracor Hydronautics

this effect. While little effect can be seen on the impedances and pressures, a major difference appears with the flow rate fluctuations. Very clearly, much higher fluctuation rates appear for the most amplified modes, and most noticeably at the upstream end of the tube. This is clear for all four highly amplified modes. Similarly, but more difficult to observe, the areas under the pressure curve seem larger for these most amplified cases. From this, obviously, more fluctuation energy is contained in the tubes for these conditions.

The method described above is an interesting tool to analyze acoustics of pipes. Its application to feed-tubes of extended nozzles has been shown and a few examples analyzed. Additional analysis and synthesis seems, however, still needed to derive engineering criteria for optimum design. In addition, the case of large fluctuations, which are out of the scope of the present method, need to be considered and are of practical interest for self-resonating, cavitating STRATOJETS. 
Tracor Hydronautics

3.0 GENERAL FORMULATION OF VORTEX BUBBLE RING PROBLEM

\subsection{Introduction}

The shear layer between a submerged jet and the surrounding fluid has the tendency to organize in large coherent structures. This has been observed for air jets discharged in air [8] as well as for water jets in water [1]. Figure 15, a picture taken in Tracor Hydronautics' High Speed Channel, illustrates this structuring. In a liquid, microbubbles in suspension collect at the centers of the shear layer microvortices and initialize the vaporization of the liquid if the pressures are sufficiently low. The cavitating jet then appears as a collection of filaments distributed over the jet shear layer. These filaments are ranđomly oriented but have the tendency to regroup at regular intervals. This phenomenon is well known in aerodynamics $[8,9]$, and the frequency of formation of these structures, $f$, corresponds to a strouhal number, $S_{d}$, close to 0.3 or its first integer multiples:

$$
s_{d}=f d / v
$$

where $d$ and $V$ are the jet diameter and velocity.

If the jet is excited at the natural structure formation frequency, then the structuring is dramatically enhanced. A cylindrical jet organizes in vortex rings. Under cavitating conditions, the rings are filled with vapor. The circulation of one of those rings is much larger than that of a vortical filament existing in a nonorganized jet. As a result, cavitation inception is much earlier, and cavitation intensity is much stronger for an excited jet. This explains our interest in studying these excited jets and the dynamics of the vaporous bubble rings. 
Tracor Hydronautics

Ring vortices have been studied extensively since the nineteenth century. Polkington [10] and Larmor [11] modelled intermolecular space as a perfect fluid, ether, where toroidal cavities enclosing vortices were distributed. Considering the case where the characteristic dimension of the torus section, $R_{0}$, is small compared to the overall ring radius, $A_{0}$, they derived an expression for the translation velocity of the ring, considering the interface as a free surface. Kelvin, in an appendix to a publication by Helmholtz [12], addressed the same question when the fluid in the torus is animated by a solid body rotation while the fluid outside the ring is treated as perfect. This expression was redemonstrated using energy considerations by Lamb [13]. The study of ring vortices was revitalized in the sixties for aircraft wakes modelization. Ting and Tung [14] calculated the ring translation velocity using matched asymptotic expansions. They assumed that all vorticity was concentrated in the "viscous core" which constituted the inner region, while the fluid in the outer region was assumed inviscid. We present in the following a similar analysis for a gas and vapor-filled ring. Surface tension at the liquid-gas interface is taken into account, as well as a compression expansion law of behavior of the gas.

\subsection{Problem Formulation}

In the two following chapters ( 4 and 5 ), we will consider the static and dynamic equilibrium of a vortex bubble ring with the assumption usually accepted for a spherical bubble. The liquid is assumed inviscid, inertial effects at the bubble-liquid interface being considered predominant when compared with the viscous effects. Fluid compressibility, as well as gas diffusion and heat transfer at the interface, are neglected since their effects predominate at different time scales. Surface tension and the presence of non-condensable 
gas are, however, accounted for. In what follows it is assumed that the ring is initially animated by vortical motion of circulation, $\Gamma$, and that it is at equilibrium with the surrounding fluid. We will seek the translation velocity of the ring at equilibrium and its dynamics and deformation when subjected to a change in the ambient pressure. The fluid being assumed inviscid, and no provision for energy loss being made, the circulation will be considered time-independent.

Figure 16 presents the coordinates system used to formulate the problem. The torus is described by the generating circle (c) of center $c$, and the section ( $s$ ). In the coordinate system $(O, X, Y, Z)$ the equation of $(S)$ is $r-R(\theta, t)$. This coordinate system is tied to a point 0 on the curve $(\mathrm{C})$, and moves with the ring while conserving its orientation. In the following we use the cylindrical coordinates $(r, \theta, Y)$ shown in Figure 16 .

If we assume that the flow is irrotational and the fluid is incompressible, then the absolute velocities derived from a potential $\phi$, satisfies the Laplace equation,

$$
\Delta \phi=0
$$

This potential must satisfy, in addition, the condition of equality of the normal velocities of the fluid and the free surface of the torus:

$$
\left\{\underline{\nabla} \phi \cdot \underline{\mathrm{n}}=\left(\frac{\partial \mathrm{R}}{\partial \mathrm{t}} \underline{\mathrm{e}}_{\mathrm{r}}+\underline{\mathrm{V}}_{\mathrm{t}}\right) \cdot \underline{\mathrm{n}}\right\}_{\mathrm{r}=\mathrm{R}(\theta, t)} \text {. }
$$

$\underline{n}$ designates the normal vector to the torus surface and $\underline{V}_{t}$ is the translation velocity of the coordinate systems $(0, X, Y, Z$ ) attached to the ring. 
Tracor Hydronautics

The condition of pressure equilibrium at the surface can be written:

$$
p(\theta, t)=p_{v}+p_{g}-2 \gamma C_{m}(\theta, t),
$$

where $p_{v}$ is the liquid vapor pressure, $p_{g}$ is the pressure of the noncondensable gas, $\gamma$ is the surface tension coefficient, and $\mathrm{C}_{\mathrm{m}}$ is the local mean curvature of the torus surface.

Using the Bernoulli equation written in the moving frame $(0, X, Y, Z)$ and a polytropic law of behavior of the gas, one obtains

$$
\begin{gathered}
\rho\left\{\frac{\partial \phi}{\partial t}-\underline{V}_{t} \cdot \underline{\nabla}+\frac{1}{2}|\underline{\nabla}|^{2}\right\}_{r=R(\theta, t)}=\mathrm{P}_{\infty}(t)+ \\
-\mathrm{p}_{\mathrm{v}}-\mathrm{p}_{\mathrm{g}_{\mathrm{o}}}\left(\frac{V_{\mathrm{o}}}{V}\right)^{\mathrm{V}}+2 \gamma \mathrm{C}_{\mathrm{m}}(\theta, t),
\end{gathered}
$$

where $\rho$ is the density of the fluid, $\mathrm{pg}_{0}$ the initial gas pressure, 0 and the torus volume initially, and at $t$ and $k$ the polytropic coefficient of compression of the gas. $P_{\infty}(t)$ is the variable local ambient pressure around the torus.

The system of equations is closed if we add the at-infinity condition,

$$
\lim _{M \rightarrow \infty} \phi(M)=0 \text {, }
$$

the condition of balance of forces on the massless ring, 
Tracor Hydronautics

$$
\iint_{\text {tore }} p(\theta, t) \underline{n} d \sigma=0
$$

and the initial conditions which satisfy the stable equilibrium of the torus. This equilibrium will be the object of Section 4.

\subsection{Normalization and Expansion Procedure}

An analytical solution of the problem is not readily available in the general case where the characteristic length, $R_{0}$, of the torus section is of the same order as the overall characteristic length, $A_{0}$, of the ring. However, an asymptotic solution is possible when $R_{0}$ is very small compared to $A_{0}$. We will assume in what follows that

$$
\varepsilon=R_{0} / A_{0}
$$

is very small compared to 1 and that the problem is axisymmetrical, $\mathrm{Cz}$ being the axis of symmetry. with this assumption, the problem has two length scales. In the region close to the torus surface, let us say, in the vicinity of 0 , the characteristic length is $R_{0}$. We will call this region the "inner region." There the toroidal cavity appears as a cylinder of axis $O Y$. Far away from the torus surface, the ring appears as a circle (c). The length scale of this overall problem is $A_{0}$, and the corresponding region is called the "outer region." The introduction of these two sub-regions and the correspnding sub-problems reduced considerably the difficulty of the problem since in each region the boundary conditions are either simplified or do not necessitate the exact knowledge of the shape of the interface. 
Tracor Hydronautics

We will use, therefore, the method of matched asymptotic equations dividing the complete problem into an inner and an outer problem with a proper matching in the intermediary region. The characteristic dimensions of the problem will depend on these two regions.

\subsubsection{Normalization of the Potential}

The potential $\phi$ can be decomposed into a circulation potential, $\phi_{C}$ and a growth potential, $\phi_{g}$ :

$$
\phi=\phi_{c}+\phi_{g}
$$

The vortical motion of circulation $\Gamma$ is responsible for the potential $\phi_{C}$, while the variations of the ambient pressure control $\phi_{g}$. Both potentials satisfy the Laplace equation:

$$
\Delta \phi_{C}=0 \text { and } \Delta \phi_{g}=0
$$

This decomposition (3.8) is valid until the translation velocity of the torus relative to the fluid, $\underline{v}_{t}$, appears in the expansions. $\phi_{C}$ and $\phi_{g}$ will be normalized separately.

In the outer problem, the velocity potential $\phi_{C}$ ext is due, to the first order, to a distribution of vortices of intensity $\Gamma$ on the circle (c). Its expression is given by:

$$
\phi_{c, 0}^{\operatorname{ext}}(M)=-\frac{\Gamma}{4 \pi} \iint_{\sigma} \frac{Q M}{T \underline{Q M T^{3}}} d \sigma_{Q} .
$$

where $\sigma$ is a surface limited by the curve (c). $Q$ is a point on $\sigma$, and $M$ is a field point (see Figure 16). This potential satisfies the Laplace equation.

Let us note that it is sufficient to limit our reasoning to first order approximations, since we are only looking for 
Tracor Hydronautics

order of magnitudes for normalization purposes.

The expression (3.10) shows that the order of maqnitude of $\phi_{c}$ ext is $\Gamma / 2 \pi$.

In the inner problem, the distribution of vortices on (c) appears in the first order of approximation as a linear vortex of intensity $\Gamma$, and of axis $0 Y$. Therefore the inner circulation potential is, to the first order,

$$
\phi_{c, 0}^{\text {int }}(r, \theta)=\frac{\Gamma}{2 \pi} \theta
$$

and $\phi_{C}$ int is normalized also by $\Gamma / 2 \pi$.

The velocity potential $\phi_{g}$ is due to the volume change of the toroidal cavity. The speed of deformation of the cylinder is $R$, and its order of magnitude is $R_{0}{ }^{2} / T$, where $T$ is the time scale of the considered phenomena and will be determined later. In the inner problem, $\phi_{\mathrm{g}}$ int is the velority potential from which the deformation speed derives. However, a space-independent potential $\phi^{*}$ is to be added in order to satisfy the matching condition with the outer problem:

$$
\phi_{g}^{\text {int }}=\phi_{r}^{\text {int }}+\phi^{*}
$$

The potential $\phi_{\mathrm{r}}$ int has for dimension $\mathrm{R}_{0}{ }^{2} / \mathrm{T}$.

In the outer problem, the volume change of the toroidal cavity is represented by a distribution of sources of intensity, $D(t)$, varying with time. The expression of the first order outer potential of growth is therefore:

$$
\phi_{g, 0}^{e x t}=-\frac{1}{4 \pi} \int_{(C)} \frac{D(t)}{|P M|} d s_{p}
$$


If $D_{0}$ is the order of magnitude of $D(t)$, $\phi_{g}{ }^{\text {ext }}$ has for characteristic dimension $D_{0} / 2 \pi$.

The values of $D(t)$ and $\phi^{*}$ are obtained by the matching between the inner and the outer problem. This is obtained by writing that the limit of $\phi^{e x t}$, when $\bar{r}$ goes to zero matches the limit of $\phi$ int when $\tilde{r}$ goes to infinity. $\bar{r}$ and $\tilde{r}$ are defined by:

$$
\bar{r}=r / A_{0}, \tilde{r}=r / R_{0}
$$

The expansion of (3.13) when $M$ is very close to $P$, or $\bar{r}$ becomes very small, is:

$$
\lim _{\varepsilon \rightarrow 0} \phi_{\Im}^{\operatorname{ext}}\left(R_{0} \varepsilon \tilde{r}, \theta\right)=\frac{D_{0}}{2 \pi}\left\{\tilde{D} \operatorname{Ln} \frac{\tilde{r}}{\bar{R}}+\operatorname{Ln} \frac{\varepsilon}{8}+0(1)\right\},
$$

where

$$
D(t)=\frac{D_{0}}{2 \pi} \tilde{D}(t)
$$

The constant $\phi^{*}$ is obtained from (3.15) and has the value

$$
\phi^{*}=\frac{D_{0}}{2 \pi} \operatorname{Ln}\left(\frac{\varepsilon}{8}\right)
$$

The first order inner velocity potential $\phi_{r}, 0$ int, which satisfies the boundary condition of normal velocity $\dot{R}$ at the cavity wall is that of the growth of a cylindrical cavity and is expressed by

$$
\phi_{r, 0}^{\text {int }}=R \dot{R} \operatorname{Ln}\left(\frac{\tilde{r}}{\bar{R}}\right)=\frac{R_{O}^{2}}{T} \tilde{R} \dot{R} \operatorname{Ln}\left(\frac{\tilde{r}}{\bar{R}}\right)
$$


Tracor Hyaronautics

As a result, by matching with $(3-15), D_{0}$ and $\phi^{*}$ are obtained:

$$
\begin{aligned}
& D_{0}=2 \pi R_{0} T \\
& \phi^{*}=\frac{R_{0}^{2}}{T} \operatorname{Ln}\left(\frac{\varepsilon}{8}\right)
\end{aligned}
$$

\subsubsection{Normalization of the Pressures}

Before considering this question, let us note that the classical difficulty encountered with the potential theory of the growth of a cylindrical cavity, namely diverging pressure at infinity, is avoided here. This difficulty arises usually from the fact that the unsteady term $\phi$ grows like $\operatorname{Ln}(x)$. Here the difficulty is avoided by the fact that the imposed at-infinity pressure is only sensed in the outer problem. The at-infinity pressure in the inner is determined by the matching condition.

In the following sections, the pressure would be normalized differently for the two cases of static and dynamic equilibrium. In the static case, the ambient pressure $P_{\infty}(0)$ far away from the torus will be considered as the scaling quantity. In the dynamic case where large pressure variations are encountered, the pressures are normalized with $\Delta P$ defined by

$$
\Delta p=\max _{t \geq 0} p_{\infty}(t)-\min p_{\infty}(t)
$$

$\Delta \mathrm{P}$ measures the amplitude of the imposed pressure variations.

\subsubsection{Normalization of the Translation Velocity}

The translation velocity of the torus, $\underline{v}_{t}$ is due to the action on an element of the ring, ds, of the distribution of 
singularities on the rest of the circle line (c). These singularities are both the vortical distribution, $\Gamma$, and the source distribution, $D$, due to the volume variation of the toroidal bubble. When $A_{0}$ is very large compared to $R_{0}$, or when $\varepsilon$ goes to zero, the ring, seen from ds, appears as an infinite cylinder of axis ds. The problem reduces then in first approximation to a two-dimensional problem, and the influence of the rest of the ring on ds is null. Therefore to the order $\varepsilon^{0}$, the translation velocity of each element ds is zero. Therefore, the contributions to the translation velocity of the vortex and of the bubble growth are respectively smaller than $\Gamma / 2 \pi R$ and $\dot{R}$, characteristic sizes of the rotation and growth velocities. This information is sufficient for the moment. The exact order of magnitude of $\underline{v}_{t}$, called $v$, will be determined in section 4.

\subsection{General Expansions and Approach}

All the nondimensionalizations are summarized in the following Table 1 where all normalized quantities are of order $1, o(1)$. The quantities are expressed either with the inner variables $(\tilde{r}, \theta)$, or with the outer variables $(\bar{r}, \theta)$ and are surmounted respectively by a tilde or a bar. 


$$
\begin{aligned}
& \tilde{\phi}_{c}^{i n t}=\tilde{\phi}_{c, 0}^{i n t}+\tilde{\phi}_{c, 1}^{\text {int }} \gamma_{1}(\varepsilon)+\tilde{\phi}_{c, 2}^{i n t} \gamma_{2}(\varepsilon)+\tilde{o}\left[\gamma_{2}(\varepsilon)\right] \\
& \tilde{\phi}_{\Gamma}^{\text {int }} \quad \tilde{\phi}_{\Gamma, 0}^{\text {int }}+\tilde{\phi}_{\Gamma, 1}^{\text {int }} \beta_{1}(\varepsilon)+\tilde{\phi}_{\Gamma, 2}^{\text {int } \rho_{2}}(\varepsilon)+o\left[\beta_{2}(\varepsilon)\right] \\
& \tilde{D}=\tilde{D}^{(0)}+\tilde{D}^{(1)} \delta^{(1)}(\varepsilon)+\tilde{D}^{(2)} \delta^{(2)}(\varepsilon)+0\left[\delta^{(2)}(\varepsilon)\right] \\
& \tilde{R}=R / R_{0}=\tilde{R}^{(0)}+\tilde{R}^{(1)} \mu^{(1)}(\varepsilon)+\tilde{R}^{(2)} \mu^{(2)}(\varepsilon)+\circ\left[\mu^{(2)}(\varepsilon)\right] \text {. } \\
& \bar{A}=A / A_{0}=\frac{-(0)}{A}+\bar{A}^{(1)} v^{(1)}(\varepsilon)+\bar{A}^{\left({ }_{2}\right)} v^{(2)}(\varepsilon)+0\left[v^{(2)}(\varepsilon)\right] \\
& \left.\dot{\tilde{x}}=\dot{\tilde{x}}^{(0)}+x^{(1)}(\varepsilon) \dot{\tilde{X}}^{(1)}+0 \dot{\left[X^{(1)}\right.}(\varepsilon)\right] \\
& \dot{\tilde{z}}=\dot{\tilde{z}}^{(0)}+\zeta^{(1)}(\varepsilon) \dot{\tilde{z}}^{(1)}+o\left[\zeta^{(1)}(\varepsilon)\right]
\end{aligned}
$$

where any function of $\varepsilon$ is such as

$$
N^{(i)}(\varepsilon)=o\left[\mu^{(i-i)}(\varepsilon)\right]
$$

and where all normalized variables are of order 1. The order of magnitude of the various functions of $\varepsilon$ are determined during the analysis by the matching conditions and the application of the principle of least degeneracy [12]. The matching condition is satisfied if the terms in the expansion of the outer potential, $\phi^{\text {ext }}$ when $\varepsilon$ tends toward zero, are identical to the terms in the expansion of the inner problem, whence the two expansions are written with the same variables ( $n-m$ rule). This can be written under the simplified form 
Tracor Hydronautics

\section{$-35-$}

Expansion ${ }_{\varepsilon \rightarrow 0} \phi^{\text {ext }}=$ Expansion $\tilde{r}_{r \rightarrow \infty} \phi^{\text {int }}$

As long as the translation velocity of the ring does not appear in the expansions, the problem remains decomposable into a growth and a rotation problem. We can then resolve in parallel and simultaneously inner and outer rotation problems as well as inner and outer growth problems. The solution at successive orders of $\varepsilon$ can be done based on the following schemes, valid up to the order where the translation velocity is to be accounted for,
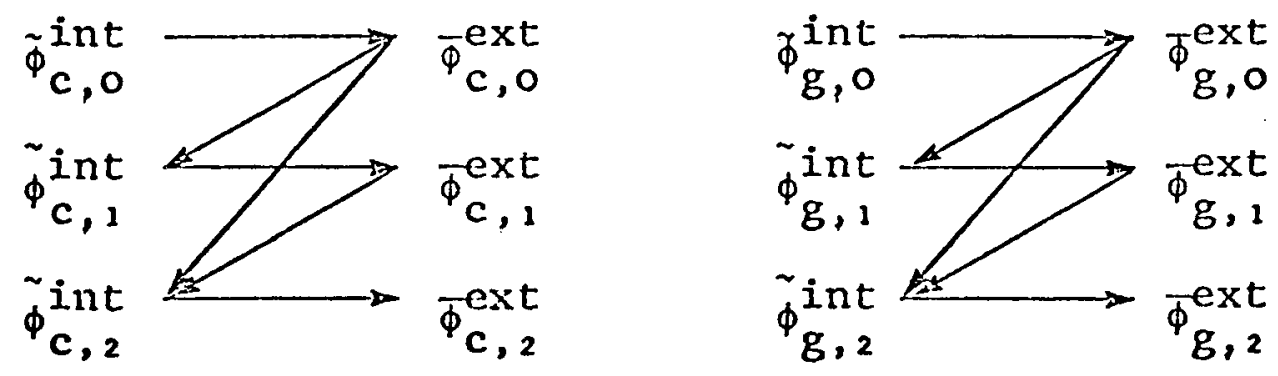


\subsection{BUBBLE RING STATIC EQUILIBRIUM}

\subsection{Translation Velocity}

In order to write nondimensional equations, let us determine the order of magnitude of the translation velocity, $\underline{V}_{t}$ of the ring in the static case where no volume change is included. In the outer problem, the velocity potential is obtained as a distribution of vortices of the circle (c). (Figure 16), the translation velocity, $\underline{V}_{t}$ is due to the action of the rest of (c) on any particular element ds. The velocity potential induced at a point $O$ of (c) by the circular distribution of vortices is obtained by the limit of $\phi_{C, 0}$ ext when $r=r / A_{0}$ goes to zero, i.e., when the field point $M$ becomes very close to $O$.

The expansion of $\phi_{C}, o^{e x t}$, written in (3.15) in the vicinity of $r=0$, in terms of the inner variables $r$ and $\theta$, is:

$$
\begin{aligned}
\lim _{\varepsilon \rightarrow 0} \phi_{C, 0}^{\operatorname{ext}} & \left(R_{0} \varepsilon \tilde{r}, \theta\right)=\frac{\Gamma}{2 \pi} \quad\left[\theta-\operatorname{Ln}(\varepsilon / 8) \frac{\tilde{r}}{2} \cos \theta\right. \\
& \left.-\varepsilon \frac{\tilde{r} \cos \theta}{2} \text { Ln } \tilde{r}+o(\varepsilon)\right]
\end{aligned}
$$

The second term of the expansion of order $\varepsilon \operatorname{Ln}(\varepsilon / 8)$ corresponds to the potential of a uniform velocity. Helmholtz theorem [12] stating that a vortical filament moves with the surrounding fluid flow, $\underline{V}_{t}$, is identical to the velocity of this flow. The value of the first order approximation of $\underline{v}_{t}$, and therefore its order of magnitude, is given by:

$$
V=\frac{\Gamma}{2 \pi R_{0}} \varepsilon \quad \operatorname{Ln} \frac{\varepsilon}{8}
$$


Tracor Hydronautics

In the following we will decompose $v_{t}$ in the $x$ and $z$ direction, and define:

$$
\begin{aligned}
& \underline{\mathrm{V}}_{\mathrm{t}}=\mathrm{v} \underline{\mathrm{V}}_{\mathrm{t}}, \\
& \tilde{\tilde{\mathrm{V}}}_{\mathrm{t}}=\dot{\tilde{x}} \underline{I}+\dot{\tilde{Z}} \underline{\mathrm{K}} .
\end{aligned}
$$

\subsection{Nondimensional Equations}

We can rewrite the general equations presented in 3.0 in nondimensional form and for the static equilibrium problem as follows:

$$
\begin{aligned}
& \Delta \tilde{\phi}_{C}^{\text {int }}=0 \\
& \left(\underline{v} \tilde{\phi}_{c}^{i n t} \cdot \underline{n}\right) \tilde{r}=\tilde{R}(\theta) \quad=\varepsilon \operatorname{Ln}(\varepsilon / 8) \quad[(\dot{\tilde{X}} \underline{I}+\dot{\tilde{Z}} \underline{K}) \cdot \underline{n}]_{\tilde{r}}=\tilde{R}(\theta) \text {. } \\
& \Omega_{s}\left\{\frac{1}{2}\left|\nabla \tilde{\phi}_{c}^{i n t}\right|^{2}-\varepsilon \operatorname{In}(\varepsilon / 8) \underline{\tilde{V}}_{t} \cdot \underline{\nabla}_{c}\right\}_{\tilde{r}=\tilde{R}(0)}= \\
& \mathscr{F}_{\mathrm{s}}-\tilde{\mathrm{p}}_{\mathrm{g}_{\mathrm{s}}}+\mathrm{W}_{\mathrm{s}}^{-1} \dot{\tilde{\mathrm{C}}}_{\mathrm{m}}(\theta) \text {. } \\
& \iint_{\text {tore }} \tilde{\mathrm{p}} \underline{\mathrm{n}} \mathrm{d} \sigma=\underline{0}, \\
& \lim _{\tilde{r} \rightarrow \infty} \tilde{\phi}_{c}^{\text {int }}=\lim _{\varepsilon \rightarrow 0} \tilde{\phi}_{c}^{\text {ext }} .
\end{aligned}
$$


Tracor Hydronautics

In this expression, the weber number, $W_{S}$, and the vorticity number, $\Omega_{S}$, measure respectively the relative effects of surface tension and pressure drop due to circulation in comparison with the ambient pressure. They are defined as:

$$
\begin{aligned}
& \Omega_{s}=\frac{p}{p_{\infty}(0)}\left(\frac{r}{2 \pi R_{0}}\right)^{2}, \\
& W_{s}=\frac{P_{\infty}(0) R_{0}}{\gamma} .
\end{aligned}
$$

Both parameters will be assumed, in what follows, to be of order unity.

In addition to the system of equations (4.4), a condition for the stability of the interface between the liquid and the bubble is imposed. This condition will consist in minimizing the surface tension energy which is proportional to the area of the interface. In order to be stable, the interface area should be minimal. For the toroidal surface with a symmetry of revolution, minimizing the area consists in minimizing the perimeter of the section (S) for a fixed surface area.

\subsection{Problem Resolution}

\subsubsection{First Order Approximation, Order $\varepsilon^{\circ}$}

The system of equations (4.4) reduces in first approximation $(\varepsilon=0)$ to: 
Tracor Hydronautics

$$
\begin{aligned}
& \frac{1}{\tilde{r}} \frac{\partial}{\partial \tilde{r}}\left(\tilde{r} \frac{\partial \tilde{\phi}_{c, o}^{i n t}}{\partial \tilde{r}}\right)+\frac{1}{\tilde{r}^{2}} \frac{\partial^{2} \tilde{\phi}_{c, 0}^{i n t}}{\partial \theta^{2}}=0 \\
& \left(\frac{\partial \tilde{\phi}_{c, 0}^{\text {int }}}{\partial \tilde{r}}\right)_{\tilde{r}=\tilde{R}}=\left(\frac{\frac{\partial}{\partial \theta} \tilde{R}^{(0)}}{\left(\tilde{R}^{(0)}\right)^{2}} \cdot \frac{\partial \tilde{\phi}_{c, 0}^{\text {int }}}{\partial \theta}\right)_{\tilde{r}=\tilde{R}^{(0)}}
\end{aligned}
$$

Similarly, the boundary condition at infinity derived from the matching condition (4.1) becomes:

$$
\lim _{r \rightarrow \infty} \tilde{\phi}_{c, 0}^{i n t}=\theta
$$

A general solution of the Laplace equation with the at-infinity condition (4.7) is given by:

$\underset{c, 0}{\tilde{\phi}_{c}^{i n t}}=\theta+\sum_{n=1}^{\infty}\left\{\cos (n \theta) \frac{c_{n}^{(0)}}{\tilde{r}^{n}}+\sin (n \theta) \frac{d_{n}(0)}{\tilde{r}^{n}}\right\}, \quad(4.8)$

Similarly, the torus section equation could be decomposed as follows :

$$
\tilde{R}^{(0)}=\sum_{n=1}^{\infty}\left\{A_{n}^{(0)} \cos (n \theta)+B_{n}^{(0)} \sin (n \theta)\right\} .
$$


Tracor Hydronautics

Without satisfying the condition of minimum surface tension energy, there is an infinite number of solutions of types (4.8) and (4.9) satisfying the boundary conditions $(4.6)$. These solutions are obtained through relations between the parameters

$a_{n}^{(0)}, A_{n}^{(0)}, b_{n}^{(0)}, B_{n}^{(0)}$

A particular elementary solution is given by

$$
\tilde{\phi}_{c, 0}^{\text {int }}=\theta
$$

This solution corresponds to a circular section of the torus, while the constants are defined as follows:

$$
\begin{aligned}
& A_{0}^{(0)}=1, B_{0}^{(0)}=0 \\
& A_{n}^{(0)}=B_{n}^{(0)}=0 \quad n \geqslant 1
\end{aligned}
$$

Any other solution of the problem would be the sum of $\phi_{C}, o^{i n t}$ given by $(4.10)$ and another potential $\phi_{t, o}$ int. This additional potential is due to any deviation of the section shape for a circle. Physically, the kinetic energy associated with $\phi_{t}, o^{\text {int }}$ balances the energy of deformation due to the surface tension. Since we are looking for a stable bubble shape, a minimization of the surface energy is sought. For constant section area (also constant volume of torus), the circular section $R(0)=1$, corresponding to $(4.10)$, is the accepted solution.

To summarize to the first order, $\varepsilon^{0}, \mathrm{R}^{(0)}=\mathrm{A}^{(0)}=1$ are solutions of all the problem equations. The balance of forces at the interface is then: 
Tracor Hydronautics

$$
\mathrm{p}_{\mathrm{v}_{\mathrm{s}}}+\mathrm{p}_{\mathrm{g}_{\mathrm{s}}}+\frac{1}{2} \Omega_{\mathrm{s}}-\mathrm{w}_{\mathrm{s}}^{-1}=1
$$

\subsubsection{Order $\varepsilon \operatorname{Ln}(\varepsilon / 8)$ - Ring Motion}

Taking into account the information obtained during the study of order $\varepsilon^{\circ}$, we can determine the values of the first functions of $\varepsilon$ appearing in the expansions (3.21). This is obtained by applying the principle of least degeneracy, which consists in keeping the maximum number of terms at the considered order of approximation. We therefore obtain:

$$
\gamma^{1}(\varepsilon)=\mu_{1}(\varepsilon)=\varepsilon \operatorname{Ln}(\varepsilon / 8)
$$

At this order, the motion of the origin of coordinates 0 , center of the circle (C), appears. This motion as stated earlier is tied to the torus translation velocity, $\underline{V}_{t}$, whose characteristic dimension has been determined in section 4.1. The system of equation (4.4) contains the following terms at order $\varepsilon \operatorname{Ln}(\varepsilon / 8)$ :

$$
\frac{1}{\tilde{r}} \frac{\partial}{\partial \tilde{r}}\left(\tilde{r} \frac{\partial}{\partial \tilde{r}} \quad \tilde{\phi}_{c, 1}^{i n t}\right)+\frac{1}{\tilde{r}^{2}} \frac{\partial^{2}}{\partial \theta^{2}} \quad \tilde{\phi}_{c, 1}^{i n t}=0 \text {. }
$$

$\left(\frac{\partial}{\partial \tilde{r}} \quad \begin{array}{c}\tilde{\phi}_{c, 1}^{i n t}\end{array}\right)_{\tilde{r}=\tilde{R}^{(0)}}=\dot{\tilde{X}}^{(0)} \sin \theta+\dot{\tilde{Z}}^{(0)} \cos \theta+\frac{\partial}{\partial \theta} \tilde{R}^{(1)}$ $(4.14)$

$-W_{S}^{-1}\left[\tilde{R}^{(1)}+\frac{\partial^{2}}{\partial \theta^{2}} \tilde{R}^{(1)}\right]=\Omega_{S}\left(\left(\frac{\partial}{\partial \theta} \cdot \tilde{\phi}_{c, 1}^{i n t}\right)_{\tilde{r}=\tilde{R}^{(0)}}+\right.$

$$
-\tilde{R}^{(1)}-\dot{\tilde{X}}^{(0)} \cos \theta+\dot{\tilde{Z}}^{(0)} \sin \theta
$$


Tracor Hydronautics

The matching condition with the outer problem gives at this order of approximations the following condition:

$$
\lim _{r \rightarrow \infty} \tilde{\phi}_{c, 1}^{\text {int }}=-\frac{\tilde{r}}{2} \cos \theta .
$$

A general solution accounting for (3.38) can be written as

$$
\tilde{\phi}_{c, 1}^{\text {int }}=-\frac{\tilde{r}}{2} \cos \theta+\sum_{n=1}^{\infty}\left\{c_{n}^{(1)} \frac{\cos \left(n^{\theta}\right)}{\tilde{r}^{n}}+d_{n}^{(1)} \frac{\sin n \theta}{\tilde{r}^{n}}\right\} .
$$

and the order $\varepsilon \operatorname{Ln}(\varepsilon / 8)$ of the surface equation can be decomposed as follows:

$$
\tilde{R}^{(1)}=\sum_{n=0}^{\infty}\left\{A_{n}^{(1)} \cos (n \theta)+B_{n}^{(1)} \sin (n \theta)\right\}
$$

The relations between $A_{n}(1), B_{n}(1), C_{n}(1)$, and $a_{n}(1)$ are obtained by using these expansions in the kinematic and dynamic equations at the bubble surface (4.14):

$$
\left.\begin{array}{c}
c_{n}^{(1)}+B_{n}^{(1)}=0, \\
n c_{n}^{(1)}+B_{n}^{(1)}\left[1+\frac{W_{s}^{-1}}{\Omega_{s}}\left(n^{2}-1\right)\right]=0 ; \\
d_{n}^{(1)}-A_{n}^{(1)}=0, \\
n d_{n}^{(1)}-A_{n}^{(1)}\left[1+\frac{W_{s}^{-1}}{\Omega_{s}}\left(n^{2}-1\right)\right]=0
\end{array}\right\}
$$


Tracor Hydronautics

This system has an infinite number of solutions such that

$$
\begin{aligned}
& c_{n}(1)=-B_{n}{ }^{(1)} \\
& d_{n}(1)=A_{n}(1)
\end{aligned}
$$

provided that the following relation between $\Omega_{S}$ and $W_{S}$ is satisfied:

$$
\Omega_{s} w_{s}=n+1, \quad n>1
$$

Otherwise all the constants are equal to zero, the circular section of the torus is the only solution, and

$$
\begin{array}{r}
c_{n}^{(1)}=d_{n}^{(1)}=A_{n}^{(1)}=B_{n}^{(1)}=0 ; \\
\dot{\tilde{Z}}(0)=-\frac{1}{2}, \quad \dot{\tilde{x}}^{(0)}=0
\end{array}
$$

This corresponds also to minimizing the energy of deformation of the surface.

Therefore, to this order the only correction to order $\varepsilon^{\circ}$ is a uniform motion of the ring. No corrections appear here to the inner potential which moves with the ring center, or to the outer potential when its expression is given with the relative variables (time-independent)

$$
\tilde{\phi}_{c, 1}^{\text {int }}=\bar{\phi}_{c, 1}^{\text {ext }}=0
$$

The fact that at this order no deformation of the moving ring section is present shows that the vaporous ring entrains with it some neighboring fluid. This result is classical for viscous vortical rings [13] and extended here for vaporous ings. 
Tracor Hydronautics

\subsubsection{Order $\varepsilon-$ Section Deformation}

The consideration of the various expansions and the application of the principle of least degeneracy show that the "second" order corrections function of $\varepsilon$ are as follows.

$$
\begin{aligned}
& \gamma_{2}(\varepsilon)=\mu_{2}(\varepsilon)=\nu_{2}(\varepsilon)=\varepsilon \\
& \chi^{(1)}(\varepsilon)=\zeta^{(1)}(\varepsilon)=\frac{1}{\operatorname{Ln}(\varepsilon / 8)}
\end{aligned}
$$

At order $\varepsilon$, the effects of the mean curvature of the ring (as opposed to an infinite cylinder) appear in the expansions of $\Delta \phi$ int and $C_{m}(\theta, 0)$. The inner potential of order $\varepsilon$ satisfies then the following equations:

$$
\begin{aligned}
& \frac{1}{\tilde{r}} \frac{\partial}{\partial}\left(\tilde{r} \frac{\partial \phi_{c}^{i n t}}{\partial \tilde{r}}\right)+\frac{1}{\tilde{\tilde{r}}{ }^{2}} \frac{\partial^{2}}{\partial \theta^{2}} \tilde{\phi}_{c, 2}^{\text {int }}=-\left(\frac{\partial \tilde{\phi}_{C, 0}^{i n t}}{\partial \tilde{r}} \sin \theta+\frac{1}{\tilde{\tilde{r}}} \frac{\partial}{\partial \theta} \tilde{\phi}_{c, 0}^{i n t}\right) \\
& \left(\frac{\partial \tilde{\phi}_{c}^{j}, 2}{\partial \dot{\partial} \tilde{r}^{*}}\right)_{\tilde{r}=\tilde{R}^{(0)}}=\stackrel{\ell}{X}^{(1)} \sin \theta+z^{(1)} \cos \theta+\frac{\partial}{\partial \theta} R^{(2)} \text {, } \\
& \begin{aligned}
W_{S}{ }^{-1}[\sin \theta- & \left.\left(\tilde{R}^{(2)}+\frac{\partial^{2}}{\partial \theta^{2}} \tilde{R}^{(2)}\right)\right]=\Omega_{S}\left\{\left(\frac{\partial \tilde{\phi}_{C, 2}^{\text {int }}}{\partial \theta}\right)_{\tilde{r}=\tilde{R}^{(0)}}\right. \\
& \left.-\tilde{R}^{(2)}-\dot{\tilde{X}}^{(1)} \cos \theta+\dot{\tilde{Z}}^{(1)} \sin \theta\right\} .
\end{aligned}
\end{aligned}
$$

At this order, the minimization of the interfacial area (stability condition) applies not only to an infinite cylinder but to the action torus.

The matching condition with the outer problem is obtained from the expansion (4.1) which gives at the order $\varepsilon$ 
Tracor Hydronautics

\section{$-45-$}

$\lim _{r \rightarrow \infty} \frac{i n t}{\phi_{c, 2}}=-\frac{\tilde{r} \cos \theta}{2}$ in $\tilde{r}+o(1)$.

A general solution which satisfies (4.25) is the following :

$$
\tilde{\phi}_{c, 2}^{\text {int }}=\cos (n \theta) \frac{c_{n}^{(2)}}{r^{n}}+\sin (n \theta) \frac{d_{n}^{(2)}}{r^{n}}-\frac{\tilde{r} \cos \theta}{2} \operatorname{Ln} \tilde{r}
$$

The radius $\mathrm{R}^{(2)}$ can also be expanded as:

$$
\tilde{R}^{(2)}=\sum_{n=0}^{\infty}\left\{A_{n}^{(2)} \cos (n \theta)+B_{n}^{(2)} \sin (n \theta)\right\} \quad
$$

Replacing in (4.24) one obtains a system of equations similar to $(4.18)$ for $n>1$ :

$$
\begin{gathered}
\mathrm{c}_{\mathrm{n}}^{(2)}+\mathrm{B}_{\mathrm{n}}^{(2)}=0, \\
\mathrm{n} \mathrm{c}_{\mathrm{n}}^{(2)}+\mathrm{B}_{\mathrm{n}}^{(2)}\left[1+\frac{\mathrm{W}_{\mathrm{s}}^{-1}}{\Omega_{\mathrm{s}}}\left(\mathrm{n}^{2}-1\right)\right]=0 ; \\
\mathrm{d}_{\mathrm{n}}^{(2)}-\mathrm{A}_{\mathrm{n}}^{(2)}=0, \\
n d_{n}^{(2)}-\mathrm{A}_{\mathrm{n}}^{(2)}\left[1+\frac{\mathrm{W}_{\mathrm{s}}^{-1}}{\Omega_{\mathrm{s}}}\left(\mathrm{n}^{2}-1\right)\right]=0
\end{gathered}
$$

Here again, $d_{n}(2)=-B_{n}(2)=A_{n}(2)$ is a solution if the condition $(4.20)$ is satisfied. These solutions are also discarded for stability (minimum surface energy) reasons.

For $\mathrm{n}=1$, the system of equations (4.24) becomes 
Tracor Hydronautics

$$
\begin{aligned}
& \dot{Z}^{(1)}+B_{1}^{(2)}+C_{1}^{(2)}=-\frac{1}{2} ; \\
& \dot{Z}^{(1)}-B_{1}^{(2)}-C_{1}^{(2)}=\frac{W_{S}^{-1}}{\Omega_{S}} ; \\
& \dot{X}^{(1)}-A_{1}^{(2)}+d_{1}^{(2)}=0
\end{aligned}
$$

This gives a correction of order $\varepsilon$ to the translation velocity of the ring:

$$
\begin{gathered}
\dot{\tilde{X}}^{(1)}=0, \\
\dot{\tilde{Z}}^{(1)}=-\frac{1}{4}+\frac{W_{s}^{-1}}{2 \Omega_{s}},
\end{gathered}
$$

The shape of the torus section remains, however, undetermined since we obtain an infinity of solutions satisfying

$$
\begin{gathered}
A_{1}^{(2)}=d_{1}^{(2)}, \\
B_{1}^{(2)}=-c_{1}^{(2)}-\frac{1}{4}-\frac{W_{s}^{-1}}{2 \Omega_{s}} \cdot
\end{gathered}
$$

The circular section is here again a solution and corresponds to $\left.A_{1}\left({ }^{2}\right)=B_{1}{ }^{2}\right)=0$ and to the following velocity potential correction:

$$
\phi_{c, 2}^{\text {int }}=-\frac{\tilde{x} \cos \theta}{2} \text { Ln } \tilde{x}-\frac{\cos \theta}{\tilde{r}}\left(\frac{1}{4}+\frac{W_{s}^{-1}}{2 \Omega_{s}}\right) \text {. }
$$


Tracor Hydronautics

At this order, $\varepsilon$, the outer potential $\phi_{C, 2^{e x t}}$ satisfies the Laplace equation and the following at-infinity and matching condition with the inner problem:

$$
\begin{aligned}
& \lim _{\overline{\mathbf{r}} \rightarrow \infty} \phi_{c, 2}^{\text {ext }}=0 \\
& \lim _{\varepsilon \rightarrow 0}\left\{\phi_{c, 0}^{\text {ext }}+\varepsilon \phi_{c, 2}^{\text {ext }}+\ldots\right\} \\
& \quad=\lim _{r \rightarrow \infty}\left\{\phi_{c, o}^{i n t}+\varepsilon \log \frac{\varepsilon}{8} \phi_{c, 1}^{i n t}+\phi_{c, 2}^{i n t}+\ldots\right\}
\end{aligned}
$$

These last conditions (4.34) allow for the matching of the term $c_{1}\left({ }^{2}\right) \cos \theta / r$ in the inner potential (4.32).

$\phi_{c}, 2^{e x t}$ is then obtained as a distribution of dipoles of intensity $\mathrm{C}_{1}\left({ }^{2}\right)$, of axis $\mathrm{CZ}$, on the circle (c) (Figure 1). Its expression is:

$$
\begin{gathered}
\underset{c, 2}{\phi_{c}^{e x t}=-\frac{k}{4 \pi}} \int_{(c)} \frac{P M}{\left.\frac{P M}{\mid P M}\right|^{3}} d s p \\
k=2 \pi c_{l}^{(2)}
\end{gathered}
$$

\subsubsection{Summary}

We have seen in this section that in an infinite medium with no imposed pressure change, a vaporous vortex ring moves in the direction of its axis of symmetry. Its section remains circular of constant radius up to order $\varepsilon$ ( $\varepsilon$, ratio of section radius to overall ring radius). The translation velocity is given by:

$$
\underline{V}_{t}=\frac{\Gamma}{4 \pi R_{o}}\left\{\varepsilon \quad \operatorname{In} \frac{8}{\varepsilon}\left[1-\frac{1}{2}+\frac{W_{s}^{-1}}{\Omega_{s}}\right]+o(\varepsilon)\right\}
$$


Tracor Hydronautics

Similar expressions not containing the Weber number term have been obtained by Tung and Ting [14] and Moore and Safman [18, 19] for viscous rings. In these expressions, $\varepsilon$ is replaced by the ratio of the viscous core to the ring radius. Tung and Ting [17] used a matched asymptotic approach similar to that presented here, while Moore and Safman [18] used the Biot and Savard law with a cutoff to remove the singularity. These approaches differ only in the value of the constant $1 / 2$ which appears in the expression of $v_{t}$, this being due to different evaluations of the viscous core size. Here no such problem exists since the core is well-defined as the vaporous part of the fluid. 


\subsection{COLLAPSE OF A CAVITATING VORTEX RING}

\subsection{Introduction}

When underwater cavitating jets are excited, the aspects of the cavitation field in their shear layer is observed to be modified from scraggly random quasilinear vapor vortices to structured well-organized ring vortex cavities moving along the jet periphery (Figure 17). Such behavior appeared with transducer-excited CAVIJET as well as with STRATOJETS where the CAVIJET nozzle or the feed tube are designed to produce self-excitation ( $2, \underline{20})$. The behavior of these cavitating vortex rings motivated this study, the final objective being to compare the erosive power of such rings collapsing on a target with that of equivalent scraggly random vortices. Comparative testing has already shown that STRATOJETS cut rock and perform underwater cleaning more effectively than conventional cavitating jets.

Another interest in this study stems from the observation that once a strong reentrant jet has penetrated a collapsing initially spherical bubble, the shape of the remaining compressed permanent gas, and thus of the rebounding bubble, is toroidal $[21,22]$. Also, the velocity of the reentrant jet impresses a torque on the toroidal bubble and induces a vortical motion. We suggest that, with the knowledge of the dynamics of a cavitating vortex ring in a pressure field and its dependence on the torque force, the experimental observation of the behavior of the rebounding and collapsing torus would be a means of measuring the reentering jet velocity. 


\subsection{Asymptotic Approach and Assumptions}

We recall in this section the main assumptions and the formulation of the problem presented in 3.2. We consider a toroidal bubble of overall radius, $A_{0}$, initially at equilibrium in an infinite medium where the ambient pressure at infinity is $\mathbf{P}_{0}$. The bubble is subjected to a vortical motion of circulation $\Gamma$. The fluid is assumed to be inviscid and incompressible, and $\Gamma$ remains constant in time. The bubble contains noncondensables of partial pressure $\mathrm{P}_{0}$ and liquid vapor of pressure $\mathrm{P}_{\mathrm{v}}$, which balance the external pressure on the bubble surface and the pressure due to the surface tension, $\gamma$. Now, we consider, as in the Rayleigh-Plesset problem for a spherical bubble, that the pressure at infinity (e.g., on the bubble trajectory) varies in time to take the imposed value $P_{\infty}(t)$, and we seek the bubble ring dynamics.

If the characteristic size, $R_{0}$, of the cross-section of the ring is of the same order as its overall radius $A_{0}$, the problem is rather complicated and the equilibrium shape of the cross-section is not circular. However, when

$$
\varepsilon=R_{0} / A_{0}<<1 \text {, }
$$

an asymptotic theory can be developed. In this case, a circular equilibrium cross-section shape of the torus is obtained in first approximation, considerably simplifying the problem. The existence of two length scales $R_{0}$ and $A_{0}$ allows the use of the method of matched asymptotic expansions which further simplifies the problem. In the "outer region" (macroscale $A_{0}$ ), the torus appears, in the most complicated case, as a distribution of 
singularities on a moving circle of radius $A(t)$. In the "inner region" (microscale $\mathrm{R}_{0}$ ) the torus is reduced to a cylinder of variable section $R(t)$. At high orders of approximation, deviation from the circular symmetry would also appear. In this section, we will limit ourselves to the first order of approximation where the deformation of the torus is limited to a variation of the cross-section radius $R(t)$. The motion of the vortex ring and the modification of its overall radius $A(t)$ appear only at the following orders.

The main assumptions for the model of the behavior of a toroidal bubble in a pressure field are that the fluid is inviscid and incompressible and that diffusion phenomena can be neglected. Such assumptions are common in the study of spheroidal cavities, and have been shown to be valid at least until the final stages of bubble collapse (see Reference [23] for a review study, and Reference [24] for a discussion of these assumptions). Here, with an inviscid fluid we must impose the additional condition that the circulation, $\Gamma$, is a constant. In a real fluid this assumption is valid as long as the time scale for the diffusion of viscosity, let us say $R_{0}^{2} / v$, is small compared with the characteristic time of collapse of the toroidal cavity (given in Equation (5.16)). For a pressure drop of one atmosphere and for water, the assumption turns out to be valid as long as the vapor core radius is greater than $0.1 \mu \mathrm{m}$.

The assumption of small vaporous core-to-ring-radius ratio $(E<<1)$ is interesting for several practical and physical situations. It corresponds to most cases of the observed occurrence of distinct jet structuring and also to the practical range of functioning of submerged jets in deep-hole drilling 
where the cavitation number, $\sigma$, is high and where the challenge is to induce cavitation by fluctuating the jet flow. In that case, o varies about the cavitation inception value, and the size of the vaporous ring core is small compared to the jet radius. When $\varepsilon$ grows, the model loses its validity, not only because of the subsequent complex geometry of the torus involved (i.e., big deformations of the vaporous section are expected), but also because of the increased interaction with neighboring rings in the jet.

We have limited this study to the first order approximation in powers of $\varepsilon$ in which the variation of the core radius and thus of the ring volume is the predominant effect. In the preceding section 4.0 , we have shown that the translation velocity is of order $\varepsilon \operatorname{Ln} 8 / \varepsilon$. Since the collapse velocity is of the order of unity, in first approximation the ring section remains circular and the bubble center does not move.

In a doctoral thesis to be presented by the second author [25], the stability of the circular shape has been demonstrated. The development of non-axisymmetric waves on the ring and their eventual instability, which has been observed experimentally, is not considered here and should be the subject of further investigation.

\subsection{Equations of the Problem}

With the above assumptions, the flow is potential, and to study the bubble ring dynamics one has to solve in the liquid the Laplace equation subjected to the boundary conditions at infinity (3.5), and at the torus wall, (3.2) and (3.4). In the asymptotic approach the cross-section of the ring is circular in first 
approximation and of center 0 . In addition, the boundary conditions at the bubble wall are to be satisfied only in the "inner region," while the boundary conditions at infinity apply only to the "outer region." In the "inner problem" the kinematic and dynamic conditions on the bubble surface, which is reduced to the cylinder $r=R(t)$, can be written (see Fig, 15):

$$
\begin{aligned}
& \underline{\nabla \phi}^{\text {int }}=\dot{R} \underline{e}_{r}+(\Gamma / 2 \pi R) \underline{e}_{\theta} \\
& \rho\left(\dot{\phi}^{\text {int }}+\left|\underline{\nabla \phi}_{i n}\right|^{2} / 2\right)=P_{\infty}(t)-P_{v}-P_{g}+\gamma / R,
\end{aligned}
$$

where dots denote time derivatives and $\phi^{\text {int }}$ the inner velocity potential. Once $\phi^{\text {int }}(R, t)$ is known, (5.3) determines the torus dynamics.

In the "outer problem" the collapsing vortex ring appears, in first approximation, as a distribution of sources and vortices on a fixed circle of constant radius $A_{0}$. If the unknown linear strength of the sources is $D(t)$ and $w$ the unknown intensity of the vortices, the "outer" velocity potential, pext, is determined at the point $M$ by:

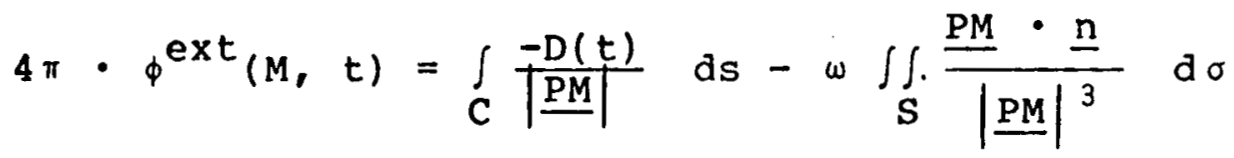

where $S$ is any surface containing the circle (C) and $\mathrm{p}$ a field point on $S$ or $(C)$. No additional terms are to be added to the expression (4) of pext, since

$$
\lim _{M \rightarrow \infty} \phi^{\text {ext }}=0
$$


Now, matching $\phi^{\text {ext }}$ and $\phi^{\text {int }}$ will allow us to determine $D(t), \omega$ as well as the needed boundary condition at infinity for

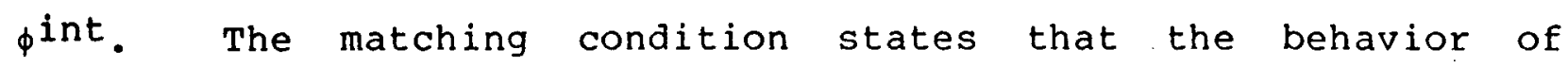
pext when the distance OM normalized with $A_{0}$ goes to zero is identical to the behavior of $\phi$ int when the distance OM normalized with $R_{0}$ goes to infinity.

\subsection{Characteristic Time of the Ring Dynamics}

Taking into account the normalizations presented in Section 3.3, the velocity potential can be written up to the order where no translation velocity of the ring or overall ring curvature appear

$$
\phi=\frac{\Gamma}{2 \pi} \tilde{\phi}_{\mathrm{C}}^{\text {int }}+\frac{\mathrm{R}_{0}^{2}}{\mathrm{~T}}\left(\tilde{\phi}_{\mathrm{r}}^{\text {int }}+\tilde{\mathrm{D}}_{\mathrm{Ln}}\left(\frac{\varepsilon}{8}\right)\right)
$$

By replacing in the dynamic equation at the bubble surface (5.3), we obtain:

$$
\begin{aligned}
& \frac{\rho}{\Delta \mathrm{p}} \frac{\mathrm{R}_{\mathrm{o}}^{2}}{\mathrm{~T}^{2}}\left[\operatorname{Ln}\left(\frac{\varepsilon}{8}\right) \cdot \frac{\partial \tilde{\mathrm{D}}}{\partial \mathrm{t}}+\frac{\partial \tilde{\phi}_{\mathrm{r}}^{\mathrm{int}}}{\partial \mathrm{t}}+\frac{1}{2}\left|\underline{\nabla} \tilde{\phi}_{\mathrm{r}}^{\text {int }}\right|^{2}\right]+ \\
& +\frac{\rho}{2 \Delta \mathrm{p}}\left(\left.\frac{\Gamma}{2 \pi R_{0}} i^{2} \underline{\nabla} \tilde{\phi}_{c}^{i n t}\right|^{2}=\left[\tilde{p}_{\infty}(t)-\tilde{p}_{v}-\tilde{p}_{g_{0}} \tilde{R}^{-2 k}+\frac{\gamma}{\Delta \mathrm{p} \tilde{R}_{0}} \tilde{C}_{m}\right]\right.
\end{aligned}
$$

The principle of least degeneracy applied to (5.7) shows that to conserve at the first order of approximation a maximum of terms, one has to choose the order of magnitude of the nondimensional terms in (5.7) as follows: 
Tracor Hydronautics

$$
\begin{aligned}
& \frac{\rho}{\Delta \mathrm{p}} \frac{\mathrm{R}_{\mathrm{o}}^{2}}{\mathrm{~T}^{2}} \text { Ln } \frac{\varepsilon}{8}=O(1) \\
& \Omega=\frac{\rho}{\Delta \mathrm{p}}\left(\frac{\Gamma}{2 \pi \mathrm{R}_{\mathrm{o}}}\right)^{2}=O(1) \\
& \mathrm{W}_{\mathrm{e}}^{-1}=\frac{\gamma}{\Delta \mathrm{p} \mathrm{R}_{\mathrm{o}}}=O(1)
\end{aligned}
$$$$
-55-
$$

The first of the equations (5.8) determines the characteristic time of the oscillations and collapse of a vaporous ring of overall radius $A_{0}$, and of section radius $R_{0}=A_{0}$, following a pressure variation of characteristic size $\triangle \mathrm{P}$ :

$$
T=R_{0}\left[\frac{\rho}{\partial p} \quad \operatorname{Ln}\left(\frac{8}{\varepsilon}\right)\right]^{\frac{3}{2}}
$$

The knowledge of $T$, and the use of (5.8) allows us to proceed with the analys's of the problem and the determination of the order of magnitua of the translation velocity of the ring.

\subsection{Translation Velocity}

Let us recall here the decomposition of the velocity potential in a circulation potential, $\phi_{C}$, and a growth potential, $\phi_{\mathrm{g}}$ (Equation 3.8). We have already discussed 
Tracor Hydronautics

$\phi_{C}$ in the preceding section 4.0. Now, the expansion of $\phi_{g}$ ext when $\varepsilon$ goes to zero in the outer problem, gives:

$$
\lim _{e \rightarrow 0} \phi_{g}^{\text {ext }}=\tilde{D}\left\{\operatorname{Ln}\left(\frac{\varepsilon}{8}\right)+\operatorname{Ln}\left(\frac{\tilde{r}}{A}\right)-\varepsilon \operatorname{Ln}\left(\frac{\varepsilon}{8}\right) \frac{\tilde{r} \sin \theta}{2 A}\right\}
$$

The motion of the vortex ring due to the volume variation is generated by the term in $\sin \theta$ in Equation (5.10) which represents a uniform velocity. Therefore, the contribution of the bubble growth to the translation velocity, $\underline{V}_{t}$ is of the order

$\varepsilon \operatorname{Ln}(\varepsilon / 8)$.

The translation velocity, $\underline{v}_{t}$, is of the order of the largest of the induced velocities by the vortex rotation and the bubble growth. That is:

$$
\mathrm{V}=\max \left[\frac{\Gamma}{\mathrm{R}^{0}} \varepsilon \operatorname{Ln}\left(\frac{\varepsilon}{8}\right), \frac{\mathrm{R}_{0}}{\mathrm{~T}} \quad \varepsilon \operatorname{Ln}\left(\frac{\varepsilon}{8}\right)\right]
$$

After accounting for (5.8), one finds

$$
V=0\left(\frac{\Gamma}{R_{0}} \varepsilon \operatorname{Ln}\left(\frac{\varepsilon}{8}\right)\right)
$$

and

$$
\begin{aligned}
& \frac{\Gamma}{R_{0}}=\frac{R_{0}}{T} \quad 0\left[\left[\operatorname{Ln}\left(\frac{8}{\varepsilon}\right)\right]^{1 / 2}\right] \\
& V=0\left[\frac{R_{0}}{T} \quad \varepsilon\left[\operatorname{Ln}\left(\frac{8}{\varepsilon}\right)\right]^{3 / 2}\right]
\end{aligned}
$$

Therefore, up to the order $\varepsilon(\operatorname{Ln} 8 / \varepsilon)^{3 / 2}$ not included, the two velocity fields due to rotation and growth are independent and orthagonal. The corresponding velocity potentials are therefore independent and could be treated separately. 
Tracor Hydronautics

5.6 Problem Solution: Bubble Ring Oscillations Equation

After using Equation (5.4) to evaluate $\phi^{\text {ext }}$ and its limit when $O M$ goes to zero (Equations 4.1 and 5.6 ), one obtains in dimensional form

$$
\begin{aligned}
D(t) & =2 \pi R \dot{R} \\
\omega & =\Gamma
\end{aligned}
$$

and, more interesting, the velocity potential in the "inner region" is determined to have as leading terms:

$$
\phi^{\text {int }}=\mathrm{R} \dot{\mathrm{R}} \quad\left(\operatorname{Ln} \frac{\mathrm{r}}{\mathrm{R}_{0}}+\operatorname{Ln} \frac{\varepsilon}{8}\right)+\frac{\Gamma}{2 \pi} \quad \theta
$$

The Log $\varepsilon$ term comes from the contribution of the whole ring to the behavior of the cylindrical portion centered at 0 . Now, taking into account $(5.16)$ and $(5.2), \phi^{\text {int }}$ and its derivative can be eliminated in (5.3) to give a differential equation for $R(t)$ which defines the bubble-ring equation. One obtains:

$$
\begin{aligned}
& \left(R R+\dot{R}^{2}\right) \text { Ln } \frac{R}{8 A_{0}}+\frac{1}{2} \dot{R}^{2}= \\
& \frac{1}{\rho}\left[P_{\infty}(t)-\frac{\rho}{2}\left(\frac{\Gamma}{2 \pi R}\right)^{2}-P_{v}-P_{g_{0}}\left(\frac{R_{0}}{R}\right)^{2 k}+\frac{\gamma}{R}\right],
\end{aligned}
$$

where $k$ is the polytropic constant defining the behavior of an ideal gas in the ring bubble.

Using the normalizations presented in section 3.3, namely, $R$ by $R_{0}$, the pressures by $\Delta P=P_{\max }-P_{0}$, and time by the 
collapse time of the bubble ring, $T$, defined in (5.9), the torus dynamic equation (5.17) can be written in nondimensional form as follows:

$$
\begin{gathered}
\left(\tilde{R} \tilde{R}+\tilde{R}^{2}\right)+\left[\tilde{\mathrm{P}}_{\infty}(\tau)-\frac{\tilde{\Omega}}{2 \mathrm{R}^{2}}-\frac{\tilde{\mathrm{P}} g_{0}}{\mathrm{R}^{2 \mathrm{~K}}}+\frac{\mathrm{W}_{\mathrm{e}}^{-1}}{\mathrm{R}}\right]= \\
\left(\operatorname{Ln} \frac{8}{\varepsilon}\right)^{-1}\left[\operatorname{Ln} \tilde{R}\left(\tilde{\mathrm{RR}}+\tilde{\mathrm{R}}^{2}\right)+\frac{1}{2} \tilde{\mathrm{R}}^{2}\right]
\end{gathered}
$$

Tildes denote nondimensional variables, and

$$
\begin{aligned}
& \tilde{P}_{\infty}(\tilde{t})=\left(P_{\infty}(\tilde{t})-P_{v}\right) / \Delta P \\
& \tilde{P}_{g_{0}}=P_{g_{0}} / \Delta P \\
& w_{e}^{-1}=\left(\gamma / R_{o}\right) / \Delta P \\
& \Omega=\left(\rho \Gamma^{2} / 4 \pi^{2} R_{o}{ }^{2}\right) / \Delta P
\end{aligned}
$$

$\tilde{\mathrm{P}}_{g_{0}}$ is a measure of the ratio of the initial gas pressure to the dynamic pressure; $W_{e}$, the weber number, measures the influence of the surface tension; while $\Omega$ defines the amount of the pressure drop due to the vortical motion relative to the dynamic pressure. If the normalization is correct, all the nondimensional terms are of order unity as long as ( $L n \varepsilon$ ) is not too big. For a very small $\varepsilon$, Equation (5.19) combined both orders, one and $\operatorname{Ln} \varepsilon$. The reasoning in section 5.4 showed that for all small values of $\varepsilon$ the characteristic time of the collapse is given by: 


$$
T-R_{0}(\rho / \Delta P)^{\frac{1}{2}}(\operatorname{Ln} 8 / \varepsilon)^{\frac{1}{2}}
$$

which differs from the Rayleigh time by a factor of $(\operatorname{Ln} 8 / \varepsilon)^{\frac{1}{2}}$, indicating a longer collapse time for a ring than for a bubble having the same diameter as the cross-section of the ring. We will see below, with the numerical solutions, that this is the case, and that (5.20) gives the proper time scaling. When $\varepsilon$ goes to zero, the right hand side of Equation (5.18) becomes negligible, and the left side gives the equation of collapse of a cavitating vortex line. If, in addition, $\varepsilon, \Omega$, $\mathrm{P}_{\mathrm{g}_{0}}$, and $\mathrm{W}_{\mathrm{e}}{ }^{-1}$ are equal to 0 , and $\mathrm{P}_{\tilde{\alpha}}(t)$ is $\underset{\sim}{\mathrm{a}}$ sudden pressure step, there is an analytic solution $\tilde{R}(\tilde{t})=\left(1-\tilde{t}^{2}\right)^{1 / 2}$.

\subsection{Numerical Solutions and Discussion}

Equations (5.17), or (5.18) can be solved numerically using a Runge-Kutta procedure. We present here a summary of the results obtained for various values of $\mathrm{P}_{\mathrm{g}_{0}}, \varepsilon$, and $\Omega$, when the cavitating bubble ring is subjected to a sudden increase of the pressure at infinity or a sudden pressure drop followed by a return to the initial pressure after a certain duration, DT. For all the cases presented, the polytropic constant of compression is taken equal to 1.4 and the weber number is 200 . The results are compared with those of a sphere of the same initial radius.

In Figure 18, the collapse of the torus when a sudden pressure jump is imposed, in absence of vortical motion and for different values of the ratio $\varepsilon$ between $R_{0}$ and $A_{0}$, is compared with that of a sphere with initial radius $R_{0}$. Nondimensional curves of $R / R_{0}$ versus time normalized by the Rayleigh collapse time, $R_{0}(\rho / \Delta P)$, show the variation of the toroidal bubble 
lifetime with $\varepsilon$. This indicates the need for the new characteristic time, $T$, different from the Rayleigh time, $T_{R}$. When the same curves are redrawn with $\mathrm{T}$ defined by (5.9), they all collapse for given values of $\mathrm{P}_{\mathrm{g}_{0}}, \mathrm{~K}$, and $\mathrm{W}_{\mathrm{e}}$.

Figure 19 shows, in the same conditions as in Figure 18, the nondimensional variations of the bubble collapse speed, $\dot{R}$, versus $t / T_{R}$. Both Figure 18 and 19 show a slower and less intense collapse of the torus relative to the sphere. This effect increases as $\varepsilon$ is decreased. However, it would be erroneous to conclude that the torus erosive effect would be less intense, since a torus of initial cross-section radius $R_{0}$ and a sphere of the same radius subjected to the same $\Delta \mathrm{P}$ do not have the same initial potential energy. One ought thus to compare both bubble shapes for the same initial volume and account for the difference in the surface of the areas subjected to the erosive power in each case. The radius of a sphere having the same volume as the torus $\left(R_{0}, A_{0}\right)$ is

$$
R_{S}=\left(\frac{3}{2} \pi \varepsilon^{-1}\right)^{1 / 3} R_{0}
$$

and the ratio of the influenced surfaces is of the order

$$
\frac{A_{T}}{A_{S}} \simeq \frac{4 \pi R_{0} A_{0}}{\pi R_{S}^{2}}=4 \varepsilon^{-1 / 3}\left(\frac{3}{2} \pi\right)^{-2 / 3}=1.4 \varepsilon^{-1 / 3} .
$$

One can see from Equation (5.21) and Figure 19 that, while the velocity of the collapse is slightly lower for the torus (about 908), the influenced area essentially compensates for this difference: the torus-influenced area is three times greater for $\varepsilon=0.1$, and four times greater for $\varepsilon=0.25$. One might then expect a higher erosive power with the bubble ring collapse. 
The influence on the collapse of $\mathrm{P}_{\mathrm{g}_{0}}$, the initial gas pressure of the bubble, can be seen in Figure 20. Normalized values of $R$ versus normalized time are represented, for the same values of $\varepsilon, \Omega$, and $W_{e}$. We observe the same influence of $P_{g_{0}}$ on the torus collapse as on the spherical bubble collapse. Small values of $\mathrm{P}_{0}$ slightly increase the collapse time. However, higher values completely modify this time indicating the need for a correction of the characteristic time which should account for $\mathrm{P}_{\mathrm{g}_{0}}$ in addition to $\Delta \mathrm{P}$. In the same Figure 20, we have drawn the analytical asymptotic solution $\left(1-t^{2}\right)$, corresponding to $\Omega=\varepsilon=W_{e^{-1}}=P_{g_{0}}=0$. We can see that the latter curve is very close to the numerical curves for $\mathrm{P}_{\mathrm{g}_{0}}=0.0$.

The effect of the rotation of the ring on its collapse is illustrated in Figure 21. The vortical motion has the same effect on the torus behavior as the noncondensable gas. Although perhaps unexpected, this result is logical once we consider Equation (5.18). It is seen that changing $\mathrm{P}_{g_{0}}$ or $\Omega$ has a comparable effect, and for $k=1$ (isothermal compression) the two parameters, $\mathrm{P}_{0}$ and $\Omega$ can be combined into a single variable. physically this is understandable since when $\Omega$ is increased local ambient pressure at the torus boundary decreases, and thus the driving collapse pressure is reduced. The same remark applies for $\mathrm{P}_{\mathrm{g}_{0}}$, since increasing, the initial gas pressure while keeping the ambient pressure constant will also reduce the driving pressure for the implosion. This observation on the influence of $\Omega$ has important consequences. For example, when attempting to increase the erosion caused by a jet, we see that increasing $\Omega$ has two opposite effects: (a) cavitation inception will appear earlier, and (b) the collapse is damped. This indicates the existence of an optimum $\Omega$ for given conditions and 
the need for an in-depth examination of the criterion required to determine this optimum value.

Let us consider now the case of a pressure drop of duration DT. We will analyze first the importance of this pressure drop. This is characterized by the parameter $\mathscr{P}$, which has been defined earlier, and which is related to the initial conditions of static equilibrium as follows:

$$
\mathscr{P}=\left[\mathrm{P}_{\infty}(0)-\mathrm{P}_{\mathrm{v}}\right] / \Delta \mathrm{P}=\tilde{\mathrm{P}}_{\mathrm{g}_{0}}+\frac{1}{2} \Omega-\mathrm{w}_{\mathrm{e}}{ }^{-1} .
$$

We can notice that $\mathscr{P}<0$ corresponds to the case where $\mathrm{P}_{\infty}<\mathrm{P}_{\mathrm{V}}$, while $0<\mathscr{P}<1$ corresponds to $\mathrm{P}_{\infty}(0)>\mathrm{P}_{\mathrm{V}}$ and $\Delta \mathrm{P}>\left[\mathrm{P}_{\infty}(\mathrm{O})-\mathrm{P}_{\mathrm{V}}\right]$. In this last case, the sudden pressure drop brings the ambient pressure below the liquid vapor pressure. The case $\mathscr{P}>1$ corresponds to $\mathrm{P}_{\infty}(0)>\mathrm{P}_{\mathrm{V}}$ and $\Delta \mathrm{P}<\left[\mathrm{P}_{\infty}(0)-\mathrm{P}_{\mathrm{V}}\right]$, in which case the ambient pressure is always above the liquid vapor pressure. These three configurations are represented in Figure 22. We will consider the two later cases, since $\mathrm{P}_{\max }<\mathrm{P}_{\mathrm{v}}$ is not practical.

Figures, 23 and 24 show the influence of the parameter $\mathscr{P}$ for two values of the pressure drop duration, DT. Figure 23 shows that for a small value of the nondimensional pressure drop duration, $D T=1$, decreasing $\mathscr{P}$ (i.e., larger $\Delta \mathrm{P}$ ) has as effect an increase of the maximum ring cross-section radius obtained and an increase of the period of oscillations. Let us notice, however, that $\mathrm{DT}=1$ is very small compared to the natural period of oscillation of a ring submitted to a sudden prrssure drop (this period of oscillation is clearly seen in Figure 24). The small value of DT did not allow the ring to achieve a stable period of oscillation during the pressure drop. In Figure 24, where DT=20, 
the low pressure duration is large enough for the ring to attain a stable behavior. Three types of behavior can then be seen depending on the comparison between $\mathscr{P}$ and 1 . For $\mathscr{P}$ greater than 1, the ring radius oscillates during the pressure drop between a maximum value and the initial value $R=1$. The natural frequency depends on $\mathscr{P}$ and decreases when $\mathscr{P}$ gets closer to 1 . The radius of the ring section continues its oscillations until the ambient pressure is brought up, then the oscillations' characteristics (amplitude, frequency) are modified. These new characteristics depend on the phasing between the ring oscillations and the pressure jump.

A different type of behavior is observed when $\mathscr{P}$ is smaller than one. In this case, the radius rapidly attains a regime of asymptotic uniform growth during the pressure drop. Following the recompression, the collapse is very intense, and its duration is only a function of the maximum radius achieved preceding the implosion. When $\mathscr{P}$ is close to one, an intermediate behavior between the two preceding ones can be observed: the ring collapses, but with less intensity than for $\mathscr{P}<1$, probably because the maximum radius is not very large. These differences can be explained further when considering the curves of static equilibrium.

Figures 25 and 26 show the influence of the pressure drop duration, DT. Depending on DT, two types of ring behavior can be distinguished. In the first case (Figure 25), the ring oscillates about its equilibrium position, $R=1$, which is frequency-dependent on DT. Similar to the case $\mathscr{P}<1$ and DT small (Figure 24), this corresponds to the configuration where the period of natural oscillations of the ring is large relative to DT. In the second case (Figure 26), the ring grows continuously 
during the pressure drop, then implodes violently following the recompression. This is followed by a rebound whose period depends on DT. The absence of damping in the oscillations is due to our assumption of an inviscid fluid.

Figure 27 shows the influence of $\Omega$, which is a measure of the relative importance of the pressure drop due to the circulation and the imposed ambient pressure drop. For a constant value of $\mathrm{DT}=20$, the influence of $\Omega$ can be compared to that of $\mathscr{P}$. An increase of the value of $\Omega$, has the effect of decreasing both the amplitude and the period of the oscillations. Small values of $\Omega$ are comparable to values of $\mathscr{P}$ smaller than one (see Figure 24 ). For instance, for $\Omega=0.05$, a DT of 20 is not large enough to observe a whole period of oscillation before the pressure jump. For the other higher values of $\Omega$, we observe oscillations between $\tilde{R}=1$ and a maximum value of $\tilde{R}$, which decreases when $\Omega$ increases.

Following the recompression, the ring behaves differently dependent on the value of the section radius, $\tilde{R}$, at $t=D T$. If $\tilde{R}$ is larger than a limiting value which is a function of the parameters $\Omega, \mathrm{P}_{\mathrm{g}_{0}}$, and $\mathrm{k}$, then a violent implosion occurs. These limiting values of $R$ correspond to the minimums of the curves of static equilibrium (Figure 28 ). If $\tilde{R}$ is smaller than the corresponding limiting $\tilde{R}$, then the section of the torus oscillates almost sinusoidally about the value $\tilde{R}=1$, and the period is a function of $\tilde{R}(D T)$. This period is the natural period of a ring of initial velocity $R(D T)$ subjected to a sudden pressure jump (Equation 5.9). The ring behavior during both phases of pressure drop and pressure jump can be explained by the fact that the circulation behaves at the same time as a spring and as a forcing function for the oscillations. 


\subsection{Curves of Static Equilibrium}

In order to explain some of the observations made above, namely the existence of a critical pressure below which for a critical radius above which) the ring section radius grows asymptotically if DT is large enough, we consider in this section the static equilibrium of a vortex bubble ring. This analysis is similar to the more classical approach for spherical bubbles.

Figure 28 gives the static equlibrium curves of a torus bubble. These curves allow the determination, for a given initial radius of the bubble, of the critical pressure below which an explosive growth (thus cavitation) is expected to occur. It is convenient to utilize a nondimensicnal representation with $\mathrm{P}^{\prime}=\left(\mathrm{P}_{\infty}-\mathrm{P}_{\mathrm{V}}\right) /\left(\mathrm{P}_{0}-\mathrm{P}_{\mathrm{V}}\right)$ as the ordinates and $R^{\prime}=R / R_{0}$ as the abscissa. The equilibrium equation is then, with an isothermal compression $\operatorname{law}(k=1)$,

$$
\mathrm{P}^{\prime}=\left(\frac{\Omega^{\prime}}{2}+\mathrm{P}_{\mathrm{g}}^{\prime}\right) / \tilde{\mathrm{R}}^{2}-1 /\left(\mathrm{W}_{\mathrm{e}}{ }^{\prime} \tilde{\mathrm{R}}\right) \text {, }
$$

where

$$
\begin{aligned}
& \Omega^{\prime}=\left(\rho \Gamma^{2} / 4 \pi^{2} R_{0}{ }^{2}\right) /\left(P_{0}-P_{v}\right) \\
& P^{\prime} g_{0}=P_{g_{0}} /\left(P_{0}-P_{v}\right) \\
& W_{e}^{\prime-1}=\left(\gamma / R_{0}\right) /\left(P_{0}-P_{v}\right)
\end{aligned}
$$

With the initial nondimensional condition:

$$
1=\frac{\Omega^{\prime}}{2}+P_{g_{0}}^{\prime}-1 / W_{e}^{\prime}
$$

e static equilibrium equation becomes: 
Tracor Hydronautics

$$
P^{\prime}=\left(1+w_{e}{ }^{-1}\right) / \tilde{R}^{2}-w_{e} e^{-1} / \tilde{R}
$$

We see that a unique parameter, $W_{e}{ }^{\prime}$, is capable of representing all cases provided that the gas compression law is isothermal, which is consistent with quasi-static modifications of the equilibrium state. Figure 28 shows $P^{\prime}$ versus $R$ for a series of values of $\mathrm{W}_{\mathrm{e}}{ }^{\prime}$. 


\subsection{Conclusions}

We have developed in this section an asymptotic theory for the behavior of a bubble ring in a variable pressure field. A dynamic equation for the oscillations of the cross-section radius of the torus was developed when the ratio, $\varepsilon$, between this radius and the overall radius is small. Static equilibrium curves of the torus were drawn which account for surface tension, noncondensable gas, and vortical motion of the bubble ring.

Numerical solutions of the vaporous torus collapse due to a sudden ambient pressure increase are presented which show the influence of $\varepsilon$, the gas content, and the circulation around the torus. A characteristic time for the collapse is derived, and the importance of the various parameters is discussed. These results indicate that a bubble ring collapse should be more erosive than that of an equivalent (same initial potential energy) spherical bubble. The vortical motion is seen to induce an earlier cavitation inception while simultaneously damping the collapse. An optimum value of the circulation should therefore be sought.

In the case of a pressure drop of duration DT followed by a recompression to the initial pressure value, different bubble ring behaviors have been uncovered. When the two pressures (initial value and minimum value) are such that a stable equilibrium of the bubble ring is possible, the toroidal bubble has a natural period of oscillation, $\mathrm{T}_{\mathrm{p}}$, which it will exhibit if DT is large enough. The behavior of the ring depends then on the relative importance of $\mathrm{T}_{\mathrm{p}}$ and $\mathrm{DT}$. If $\mathrm{T}_{\mathrm{p}}$ is larger than DT, the bubble implosion occurs only after the recompression takes place. If $\mathrm{T}_{\mathrm{p}}$ is smaller than $\mathrm{DT}$, the torus section oscillates before collapsing following recompression. The value 
of $T_{p}$ depends mostly on the amount of pressure drop, and on the relative values of noncondensable gas pressure and pressure drop due to circulation (therefore, on the parameters $P, \Omega$ and $\mathrm{P}_{\mathrm{g}_{0}}$ ). Here again, both the circulation and the noncondensables act as spring constants in the oscillatory behavior of the toroidal bubble.

When the minimum pressure is lower than the critical pressure (pressure below which no static equilibrium is possible), the radius of the torus section grows explosively during the pressure drop. When the recompression occurs, the violence of the ensuing collapse is a function of the maximum radius attained during the time DT as well as on the parameters $P, \Omega$, and $\mathrm{P}_{\mathrm{g}_{0}}$. 
6.0 SIMULATION OF THE PRESSURE FIELD DUE TO A SUBMERGED OSCILLATING JET IMPACTING ON A SOLID WALL

\subsection{Introduction}

Experimental observations of air jets show the tendency of the turbulent eddies in their shear layer to organize in large structures. Excitation of a jet with periodic acoustic signals produced upstream of the nozzle by transducers or loud speakers shows a remarkable change of the jet structure into discrete ring vortices when the excitation frequency, $f$, matches the predominant natural frequencies of the non-excited jet $[\underline{8}, 26]$. This corresponds to a strouhal number, $s_{d}$, close to 0.3 or one of its first integer multiples. (The strouhal number is defined as $s_{d}=f d / V$ where $V$ and $d$ are the velocity and the diameter of the jet.) Based on these observations, several attempts have been carried out to model a jet with a discrete distribution of vortex rings. Axially symmetric vortex rings with a viscous core have been investigated and the numerical schemes were able to reproduce satisfactorily experimental measurements or observations $[9,27]$. For instance, the fluctuating pressure field in the jet vicinity was simulated by Fung, et al. [27]. Acton [9] simulated the upstream portion of the jet with a double row of ring vortices which were allowed to interact and move freely downstream of the nozzle exit. The roll up of the vortex sheet into large axisymmetric structures was numerically demonstrated. Artificial excitation of the jet by periodically modulating the strength of the emitted vortices exhibited the same features as observed experimentally, namely the existence of an optimal strouhal number.

Under preceding contract efforts for sandia, in order to improve the erosive and cleaning power of submerged water jets in deep drilling technology, the same ideas were applied to 
cavitating jets $[2,3,20]$. Here the cavitation appears in the shear layer in randomly oriented vortex filaments in which gas and vapour cavities grow. The studies conducted at Hydronautics have shown very clearly that a cavitating jet can be organized in well defined bubble ring vortices when excited. This has augmented the erosive and cleaning power of these jets. Both external excitation with a transducer and self-induced oscillations have been considered. In the previous sections, we have modelled the growth and collapse of a toroidal bubble in an infinite medium. A dynamic equation was derived, and the importance of the various parameters investigated. The characteristic time of the collapse of the toroidal cavity and. its self-induced translation velocity were obtained. [28, 29].

This section presents one of the first steps towards the understanding of the enhancement of the cleaning effects associated with the excited jets and is mostly concerned with the interaction of an organized jet with a solid wall. Taking advantage of the numerical results obtained in previous studies and of experimental observations, one can simplify the problem by neglecting the fine structure of the jet's shear layer and assuming that the major features of the flow field are associated with the large structures. Thus, the excited jet is modelled with large vortex rings emitted from the nozzle orifice at constant time intervals which correspond to the frequency of the pulsing jet. Each of these vortex rings has in its center a gas and vapor-filled toroidal cavity, and is allowed to move and change shape under the influence of its self-induced velocity as well as the velocities induced by the other ring vortices present in the field. The presence of the wall is accounted for by applying the method of images since no viscous effects are considered. Knowing the location and characteristics of all rings at any time allows the computation of the generated pressure field. We present here the model used and some results 
Tracor Hydronautics

on the interactions of the large structures between each other and with the wall, as well as the generated pressures on several arbitrary locations on the solid wali.

\subsection{Formulation of the Problem}

Let us consider a vortex ring of overall radius $A_{0}$ and of circulation, $\Gamma$, constant in time. The core of the $r$ ing is gas and vapour filled and has a circular section of radius $R_{0}$. The surface tension coefficient at the bubble liquid interface is $\gamma$, and the initial partial gas and vapor pressures inside the bubble are $\mathrm{P}_{\mathrm{g}_{0}}$ and $\mathrm{P}_{\mathrm{V}}$. Outside the gaseous core we can assume that the liquid is inviscid and incompressible and that the flow is irrotational. Therefore, the liquid flow is potential. In order to simplify the analytical and numerical approach, we consider, as in the preceding sections, the case where the radius of the gaseous section is small compared to the torus radius and introduce the small parameter,

$$
\varepsilon=R_{0} / A_{0} \ll<1
$$

We can then consider an asymptotic approach to the problem and use the method of matched asymptotic expansions. Concerning the investigation of the bubble ring behavior, two regions of the fluid field are introduced. In the "inner region" of characteristic length $R_{0}$, the problem is concerned with the torus core and is two-dimensional, and the bubble appears as cylindrical. In the "outer region" of characteristic length Ao,

the cavity cannot be distinguished, and the problem reduces to the well-known potential problem of a circular vortex line. For this "outer problem" the expression of the stream function $\psi$ is given by Lamb [13]:

$$
\psi=-\frac{\Gamma}{2 \pi}\left(r_{1}+r_{2}\right)\left[k\left(\frac{r_{2}-r_{1}}{r_{2}+r_{1}}\right)-E\left(\frac{r_{2}-r_{1}}{r_{2}+r_{1}}\right)\right]
$$


Tracor Hydronautics

where $E$ and $K$ are complete elliptic integrals of the first and second kind respectively, and where the geometric distances $r_{1}$ and $r_{2}$ are defined in Figure 29. Similarly, the velocity potential can be written as

$$
\begin{aligned}
& \phi^{\text {ext }=} \frac{-\Gamma}{4 \pi} \int_{S} \frac{P M \cdot e_{z}}{|\underline{P M}|^{3}} d s= \\
& \operatorname{sgn}(z-z) \frac{\Gamma A_{0}}{2} \int_{0}^{\infty} e^{-k|z-z|} J_{0}(k x) J_{I}\left(k A_{0}\right) d k
\end{aligned}
$$

where $J_{0}$ and $J_{1}$ are the Bessel Functions of order 0 and 1 , and $P$ is a point of any surface $S$ limited by the circle of radius $A_{0}$.

In order to determine the self-induced translation velocity of the vortex ring, one has to solve both the inner and outer problems using a matching condition at the boundary of the two regions. For instance, when no bubble growth occurs, the behavior of the outer solution in the vicinity of the bubble wall can be obtained by expanding pext in powers of $\varepsilon$ and $L n \varepsilon$, (see Section 4.0$)$, and can be written:

$$
\begin{aligned}
\lim _{\varepsilon \rightarrow 0} \phi^{\operatorname{ext}\left(\varepsilon \hat{A}_{0} \tilde{r}, \theta\right)=} & \frac{\Gamma}{2 \pi} \quad \theta-\varepsilon \operatorname{Ln}\left(\frac{\varepsilon}{8}\right) \tilde{r} \cos \theta \\
& -\varepsilon \frac{\tilde{r} \cos \theta}{2} \operatorname{Ln} \tilde{r}+0(\varepsilon),
\end{aligned}
$$

where the nondimensional distance, $r$, from the buhble center to the field point $M$ is defined by:

$$
\ddot{r}=r / A_{0}=r / \varepsilon R_{0}
$$

Expression $(6-4)$ is used for writing the boundary conditions at infinity for the inner problem, and contains most of the 
Tracor Hydronautics

information needed to compute the bubble translation velocity. Besides the vortical motion which is given by the first order term, a uniform velocity appears to the following order, $\varepsilon \operatorname{Ln}(\varepsilon / 8)$. More complicated terms appear at the higher orders. If the bubble ring is not isolated, the second term is modified by an additional constant multiplying $r \cos \theta$.

In the "inner problem," one has to solve the Laplace equation along with kinematic and dynamic boundary conditions on the bubble wall (see section 4.0). After using the boundary condition at infinity derived from (6.4), one finds, [29] that the circular shape of the bubble section is a stable solution up to the order $\varepsilon$, and that the self-induced velocity of the ring is

$$
\underset{\text { self }}{\mathrm{V}_{-}}=\frac{\Gamma}{4 \pi \mathrm{R}_{0}}\left\{\frac{\varepsilon}{\overline{\mathrm{A}}}\left[\operatorname{Ln} \frac{8 \overline{\mathrm{A}}}{\varepsilon}-\frac{1}{2}+\frac{\mathrm{W}_{\mathrm{S}}^{-1}}{\Omega_{\mathrm{S}}}\right]+0(\varepsilon)\right\},
$$

where $A$ is the nondimensional overall ring radius at the considered time. $\mathrm{W}_{\mathrm{S}}{ }^{-1}$ is the inverse of the weber number and $\Omega_{s}$ the circulation number measuring respectively the relative importance of the surface tension and the pressure drop due to circulation relative to the ambient pressure. These are written as :

$$
\begin{aligned}
& W_{s}^{-1}=\gamma /\left(R_{0} P_{\infty}\right) \\
& \Omega_{s}=\rho\left(\Gamma / 2 \pi R_{0}\right)^{2} / P_{\infty}
\end{aligned}
$$

A similar expression has been obtained for vortex rings with a viscous liquid core. In that case, $\varepsilon$ is replaced by $\delta$, the ratio between the core size and the torus radius, $A$, the surface tension term, is null and the constant $1 / 2$ is replaced by $1 / 4$ [18]:

$$
v_{\text {viscous }}=\frac{\Gamma}{4 \pi R_{0}}\left\{\delta\left[\operatorname{Ln} \frac{8}{\delta}-\frac{1}{4}\right]+0(\delta)\right\} .
$$




\subsection{Numerical Procedure and Remarks}

In order to simulate the behavior of the jet and its interaction with the wall, we used the following procedure. At $t=0$, a ring is emitted from the location $x=0$ and is allowed to move under the influence of its self-induced velocity. The velocity due to the ring image is to be added when a solid wall is present. Subsequently at the discrete instants $t_{n}=n / f$, ( $f$ is the jet oscillation frequency and $n$ an integer number), an additional ring is emitted and is allowed to interact with the rings already in field and their images. At every time step the locations of all the rings are computed, as well as their translation velocities, which in turn determine their subsequent positions. The self-induced velocity of any ring is computed using Equation (6.6), while its translation velocity due to the other rings and the ring images is derived using Equations ( 6.2 ) or (6.3) since the analysis shows that the translation velocity of the bubble ring due to an imposed incoming flow is equal to the fluid velocity at its location.

As expected, the behavior of the emitted rings is at first unsteady and does not represent the steady jet behavior. However, after certain computation time has elapsed the system becomes steady and a periodic behavior of the various emitted rings is exhibited. Once the location of all the rings is determined, the pressure at any location can be obtained using the Bernoulli equation:

$$
p(M)-p_{\infty}=-\rho\left[\frac{\partial \phi^{e x t}}{\partial t}+\frac{1}{2}\left|\nabla_{\phi}^{\text {ext }}\right|^{2}\right]
$$

where pext is given using (6.3).

Let us note that in the procedure and the analytical expressions presented above we have neglected both the influence of the nozzle and the bubble growth. The first effect is too 
Tracor Hydronautics

complicated to account for with accuracy at the present time and will constitute a future subject of investigation. The bubble growth effect which we have studied earlier (section 5.0 and Reference [28]) can be neglected if the variation of the local pressure around the bubble ring and the influence on the vortex ring motion are small compared to $\varepsilon$, the highest order term considered. in the above expressions. This is true as long as the distance between two tori is of higher order of magnitude than $\varepsilon$, (i.e., as long as the distance is greater than the section radius). This assumption, as we will see below, is valid in all the cases studied and fails only when the vortex ring becomes very close to the wall. In fact, in that case not all assumptions are valid and viscous effects on the wall must be considered.

\subsection{Results and Discussion}

In all results presented below, distances are normalized with the nozzle radius, $A_{0}=d / 2$, and velocities with the self-induced velocity of an isolated ring, $v_{s} f_{0}$. For comparison purposes, Figure 30 shows the behavior of the simulated jet in absence of a solid wall. In this case, a steady periodic solution appears after about seventeen time steps. Then the multiple rings exhibit similar behavior to two equal rings, namely successive threading and overpassing of one ring through the other. Here all rings emitted at an odd number normalized time follow the same path, while all even numbered ones follow a different pain. At a given subsequent time, the rings are located as those numbered in the figure. The arrowed lines in the figure join two successive rings and show that the closest to each other these rings get is at the moment they pass each other and that even then they stay at a distance of order unity. 
Figure 31 and 32 show the same type of trajectories representation but in presence of a solid wall and for two reduced frequencies $\bar{f}=1$ and 2 (or $s_{d}=f V_{s e l f} / d=0.5$ and 1). In both cases the standoff distance, $\bar{z}=z / A_{0}$, is equal to 5. The importance of the strouhal number on the jet behavior is very clear in this example. Obviously, the optimum location of the wall for maximum erosion and cleaning power which corresponds to either maximum tangential velocities on the wall or minimum pressure is not the same for both cases. In Figure 31, two distinct trajectories for odd and even numbered rings are seen, as in the free jet case. This is not the case for $s_{d} \sim 1$,

Figure 32, where all even numbered rings and one out of two odd numbered rings follow one trajectory, while the other half of the odd number rings follow another. In both cases the steady solution was attained much faster than for the free jet after about seven time steps. We can notice also that in both cases two successive rings are always much further apart than $\varepsilon$. However, their distance to the wall becomes of this order at a radial distance to the jet center, $\bar{x}$, of about 2.5 .

Figures 33 and 34 show the tangential velocities induced on five different locations on the solid wall versus time. The spikes correspond to the passage of a vortex ring. It is interesting to notice that both the amplitude and the frequencies of the locally sensed velocity spikes are dependent on the point of observation. The same observations can be made concerning the generated pressures represented in Figure 35 and 36 . The occurrence of the pressure and velocity spikes for both frequencies are very much correlated, as well as their amplitudes. Both indicate that the predominant term is the steady term $|\nabla \phi|^{2}$, and that the major factor with our assumptions appears to be the distance at a given time between the ring and the wall. This explains why the highest negative pressure peaks are at an optimum (in the analyzed range) value of $x$. This 
Tracor Hydronautics

predominance of the term $|\Delta \phi|^{2}$ is due to the neglect of the bubble radius variation and thus of the important corresponding $\partial \phi / \partial t$ terms.

Both the high intensification of the tangential velocities and of the suction pressures on the wall is due to the passage of organized structures and indicate a significant enhancement of the cleaning capability of water jets through excitation. This is possible through an increased shearing action of the jet on the solid chips on the wall as well as increased lifting forces. These first results of the developed program should be refined through accounting for the bubble volume change near the wall. They should also be extended to obtain relationships between Strouhal numbers, optimum standoff distances, and maximum pressure drop on the wall. The same numerical program can be used for noncavitating vortex rings by replacing Equation (5.6) by Equation (6.9). 


\subsection{STUDY OF "LOW-SIGMA" OSCILLATING NOZZLES}

As already mentioned in section 1.0 , the ultimate objective of the investigation conducted under the present contract is the development of high-pressure pure jet bits. These high-pressure jets would operate under low cavitation number conditions during the early stages of the drilling process. For instance, for a 10,000 psi jet, the cavitation number, $\sigma$, would be less than 0.25 for depths less than 5,000 feet. This stresses the need to develop "low-sigma" self-resonating nozzles in order to take advantage of jet structuring for as shallow a depth as possible at a given velocity, or as high a velocity as possible at a given depth.

Experimental observations and measurements have shown that our "first-generation" STRATOJETS show strong structuring and entertain high pressure and velocity oscillations for cavitation numbers ranging from 0.25 up to about 4 . Figure 37 illustrates this structuring for a cavitation number of 0.56 . The three pictures in this figure compare the appearance of the jet interface for three plexiglass nozzles. The first nozzle is a conventional smith nozzle, while the second one is a CAVIJET-shaped nozzle with a very sharp edge (almost no lip thickness). The third nozzle is a STRATOJET nozzle with the same internal shape as the preceding nozzle but with a lip designed so that a feedback mechanism exists and excites resonance in the upstream organ-pipe which is the same for the three nozzles. While the first two nozzles show some tendency toward structuring, a little more pronounced for the CAVIJET shape, the third nozzle shows very strong structuring. One can also notice that the cavitation number for the first two nozzles has to be dropped to almost half its value for the self-resonating nozzle in order to observe cavitation in the shear layer. 
Tracor Hydronautics

When the cavitation number is further lowered, the ringshaped cavities become fatter, as illustrated in Fiqure 38, and as a result their interdistances become small relative to their size. Obviously, our analytical model developed earlier then loses its validity. Ultimately, cavitation extends to a large volume of the jet and into the nozzle lip. As a result, the feedback mechanism at the nozzle orifice between the natural oscillations of the jet and the natural modes of the organ pipe vanishes. The self-resonance is then lost, and the jet has the classical appearance of flashing jets as shown in Figure 39. This illustrates the difficulty in designing a "low-sigma" oscillating nozzle.

\subsection{Preliminary Analysis of Feedback Mechanism}

The following preliminary analysis and discussion is useful to explain some of the ideas tested in order to design a "low-sigma" self-resonating nozzle. Two types of sources of feedback can be distinguished. The first type of excitation of oscillations in the organ pipe is acoustical and is related to the propagation of sound waves generated downstream of the nozzle. The second type is hydrodynamic and is related to the nozzle lip - jet interaction.

One major source of acoustical excitation is the growth and collapse of cavities in the jet shear layer. The generation of these cavities below cavitation inception is reflected by a sensible increase of pressure oscillations in the feed organ pipe. This phenomena is fundamentally related to sound speed and sound propagation in the medium. As already described in section 2.2, the sound speed is strongly dependent on the presence of bubbles in the liquid. The same is true concerning the attenuation of wave propagation. Consequently, when the cavitation number is lowered, the transmission of 
signals from the collapsing cavities upstream into the nozzle is significantly reduced.

The nozzle lip - jet interaction phenomenon is illustrated schematically in Figure 40. Our knowledge of this phenomenon is for the moment limited and rather empirical. Experimental observations and numerical simulation of free-jet (no nozzle interaction) [9] have shown the tendency of the jet surface to deviate from its initial cylindrical shape. Wavy fluctuations appear near the nozzle exit, then develop into large instabilities of rotating structures (rings). In the presence of a surrounding wall, (i.e., nozzle lip), these oscillations and instabilities are obviously modified by the wall presence. The pressure field in the confined space between the jet and the nozzle walls then becomes of great importance. A low pressure region develops, which attracts the jet toward the wall. This modifies the "end condition" for the flow across the nozzle (e.g., the discharge coefficient). As a result, the jet surface returns toward its initial position, and the cycle is repeated. If this occurs, an oscillatory mechanism is generated which could lead to resonance with the upstream oscillator (organ-pipe). This is illustrated in Figure 40 .

The formation of a large structure develops earlier in the presence of the wall. A region of internal recirculation, $A$, is created. Later, in the second phase of the process, the big structure moves downstream, allowing the escape of the entrapped volume $A$ and the regeneration of entrained liquid into the jet shear layer. A probable mechanism for the loss of feedback when the cavitation number becomes too low, is the vaporization and/or gas filling of the vortical region $A$. A super cavity is then formed at the nozzle exit. If this cavity possesses steady characteristics, then no mechanism is present to provide an excitation to the organ-pipe resonator. The presence of such a "super cavity" also prevents the transmission of any downstream acoustic waves into the nozzle. 


\subsection{Concepts for "Low-Sigma" Self-Resonating Nozzles}

Based on the above discussion, three types of nozzles were considerec as candidates for "low-sigma" self-resonating nozzles: a sharp edged (very short lip) nozzle, a very long orifice nozzle, and a pulser-fed nozzle (see Figures 41, 42). This choice is based on the following concepts. The first nozzle was chosen to avoid the formation of a "super cavity," the second nozzle to control it, while the third one, already described in earlier reports $[2,3]$, provided excitations upstream of the nozzle in a noncavitating region.

The first type of low sigma nozzles (Figure 41 a) avoids the formation of a super cavity at the nozzle orifice by eliminating the presence of a recirculation region, such as region $A$ in Figure 40 . This minimizes the interaction between the jet and the nozzles, and as a result, the hydrodynamic feedback mechanism. The only remaining possible source of feedback is acoustical. Therefore, one would expect this concept to work for lower cavitation numbers than the first generation of STRATOJETS, but still to be cavitation number-limited. Also, the amplitudes of the fluctuations are expected to be lower than for the first generation STRATOJET which takes advantage of both sources of excitation. The acoustical source is due to the collapse of the cavities downstream of the nozzle. Since the formation of these cavities is intimately related to the presence of vortical filaments in the shear layer, their frequency of occurrence is the natural frequency of the jet. The impulses generated by the implosion occur therefore at the jet characteristic Strouhal number. At moderate cavitation numbers, this characteristic Strouhal number, $\mathrm{S}_{\mathrm{d}}{ }^{*}$ is about 0.3. However, as observed by Jorgensen [30] while studying cavitating jet joise, $\mathrm{S}_{\mathrm{C}}{ }^{*}$ decreases significantly with the cavitation number. One has therefore to account for this while designing a low-sigma self-resonating nozzle. 
The second type of low-sigma nozzles (Figure $41 \mathrm{~b}$ ) attempts to harness the super cavity formed at the nozzle orifice and to use it as a hydrodynamic source of excitation. Here one tries to take advantage of the knowledge on supercavitation to design a good nozzle. A super cavity formed behind a foil, for instance, has a characteristic length and oscillates at a characteristic frequency dependent on the cavitation number. The same is expected for the axisymmetric cavity represented in Figure $41 \mathrm{~b}$. The feedback mechanism would then be related to the oscillations of this cavity, which have to be matched to the organ pipe natural frequency. One important matter of concern with this type of nozzle is the susceptibility of erosion of the long portion of diameter $d_{1}$.

The PULSER-FED solution (Figure 42), if acceptable for practical applications, seems at first glance to be the nost promising concept. This solution does not count on any feedback mechanism downstream of the nozzle exit, but on oscillations in the Helmholtz chamber located upstream of the nozzle feed tube. The sizes of the openings of this chamber can be large enough to reduce velocities in the chamber and avoid cavitation. In these conditions, resonance can be easily achieved in the chamber at a local strouhal number (based on chamber entrance diameter and corresponding velocity) of 0.3 or one of its multiples. These oscillations can be strongly transmitted downstream if there is an impedance match with the feed tube to the nozzle. If, in addition, the frequency of oscillation matches the natural cavitation number-dependent oscillations of the jet, then a strong self-resonating low-sigma nozzle can be obtained.

\subsection{Experimental Results}

The results presented below do not constitute a thorough study of the three types of nozzles described above. Instead, 
Tracor Hydronautics

they analyze the feasibility of the concepts described in the preceding section. Experimentation has shown, in addition, the importance at low cavitation numbers of the presence of a nearby impacted wall on the measured oscillations. This influence is illustrated in the summary figures shown below. Two other geometric characteristics were seen to be important. The first, the organ-pipe length $\mathrm{L}$, is related to a parameter mentioned above, i.e., the characteristic strouhal number of the jet. The second is the contraction ratio $D / d$ (see Figure 41). Better results were obtained for the sharp-edge nozzle for bigger contraction ratios. Relatively low frequencies are seen to predominate at lower sigmas.

Figure 43 compares, for sharp-edged nozzles, the frequency spectra at two different cavitation numbers. The shift at low sigmas toward lower frequencies and the appearance of a peak low frequency $(5.5 \mathrm{kHz})$ is evident in this figure. The choice of an organ-pipe length corresponding to this frequency ( $\mathrm{L} \sim 5.8$ in.) did not, however, have an amplification influence on the pressure fluctuations. As can be seen in Figures 44 and 45, the influence of standoff distance is predominant. Figure 44 presents the pressure fluctuations measured in Tracor Hydronautics High Pressure Cell far from the nozzle (which explains the low numbers) for different values of the ambient pressure, and thus, of the cavitation numbers. Compared are a few standoff distances, $x$, and two organ-pipe lengths, $L$. $x=0.5$ in. seems to give the highest fluctuations in the low-sigma region. Figure 45 shows a dramatic improvement at low sigmas when the contraction factor was increased from 1.5 to 3.15. Oscillations peak at a sigma as low as 0.02. Another important observation is that at a higher cavitation number $(\sim 0.5)$ the high contraction nozzle performs poorly in comparison with the lower contraction nozzle. This reinforces the idea that shortening the nozzle lip relative to jet 
Tracor Hydronautics

diameter, which is the effective result of increasing the contraction ratio, increases feedback at low sigmas. simultaneously, this reduces nozzle lip - jet interactions and as a result harms hydrodynamic feedback at higher cavitation numbers.

Figure 46 shows a typical result obtained with the long orifice design. Here again, the short standoff distance, $x-0.5$ in. seems to be very beneficial to oscillations. The fluctuations are lower than for the sharp-edged nozzles at the lower sigmas, but the long-orifice nozzle resonates more strongly in a larger band of values of sigma. The difficulty with the long-orifice nozzles is the need for a visualization of the flow inside the orifice for guidance toward optimization. After a series of tests, the orifice was seen to be eroded.

Figure 47 shows the pressure fluctuations in the feed pipe for a PULSER-FED nozzle and at two different cavitation numbers. One can observe that while the values of the relative pressure fluctuations are lower when the cavitation number is lowered from 0.7 to 0.1 , the variations with the jet velocity conserve the same shape. More interestingly, discrete frequencies can still be observed but are lower than for the high cavitation number case. Figure 47 shows that the corresponding strouhal numbers are halved. The existence of discrete structuring at low cavitation numbers can be visually observed. Discrete "puffs" of bubble clouds can be seen exiting from the nozzle at a fixed rate. Figure 48 shows still pictures of this structuring for two low cavitation numbers $\sigma \approx 0.01$ and $\sigma \approx 0.07$. In both cases, the strouhal number is as low as .08 or about a quarter of the classical value $s_{d}{ }^{*}$ of 0.3 .

We have investigated in this section the feasibility of obtaining low-sigma self-resonating nozzles. The results are 
Tracor Hyảronautics

$$
-85-
$$

encouraging but show the need of a more detailed analysis and observation of the cavities (fat rings or clouds of bubbles) in the jet shear layer at these low values of sigma conditions. 


\subsection{CONCLUSIONS AND RECOMMENDATIONS}

A variety of experimental and analytical tasks have been performed to date to develop cavitating jets which are suitable for enhancing the performance of deep-hole drill bits. Several self-resonating nozzle types, STRATOJETS, were developed. These nozzles produce passive oscillations which cause the jet to structure into discrete ring vortices and therefore possess incipient cavitation numbers two to six times higher than obtained with conventional CAVIJET nozzles. The present phase of the study was aimed at gaining a better fundamental understanding of the phenomena involved in self-resonating nozzles. The objectives were to achieve further improvements, to understand scaling laws, and to move towards the development of a pure jet bit. In this report, we have summarized the achievements towards the modelling of the basic phenomena involved. We have developed analytical and numerical techniques which enabled us to describe the various aspects of the problem. This could be summarized as follows:

(a) A thorough analysis of the acoustic dynamics of the supply system to the nozzle was achieved. Natural and forced mode oscillations were studied, and damping and amplification factors included. Such analyses allow the design of the optimum feed-tube from the acoustical viewpoint.

(b) A unique model of bubble ring growth and collapse was developed. Similar to known theories on spherical bubble oscillations, this model resulted in a knowledge of the characteristic period of oscillation of a bubble ring, as well as its dynamics in a pressure field. Conclusions on the relative importance of the rotational velocity around the ring 
Tracor Hydronautics

(circulation) and the jet velocity for maximum collapse intensity (and therefore erosive power) were derived.

(c) A numerical model based on distributed ring vortices in the jet shear layer was implemented in order to simulate the cavitating self-resonating jet behavior.

(d) From the above models an understanding of the flow field, the cavities' motion, and the pressure field generated by a submerged self-resonating STRATOJET was achieved.

(e) Visualizations, acoustic measurements, and photography were used to assess the structuring of the jets and to observe bubble ring formation and behavior.

(f) A series of new types of STRATOJETS was assessed in order to achieve acceptable structuring and resonance at a low cavitation number, siqma. This feasibility analysis constitutes a preliminary step toward the development of a pure jet bit.

From this phase of the study, we can make the following general conclusions:

(1) Within the constraints of a qiven bit design, optimal supply system design can be obtained by using the acoustical analysis derived in this program.

(2) Bubble ring collapse could be intense enough to explain the observed high erosion rates with STRATOJETS. The passage of these ring vortices near a wall could generate high suction pressures to achieve chip lift.

(3) Higher erosivity could be achieved if an optimum between rotation (circulation) and axial (jet) velocity can be achieved. 
Tracor Hydronautics

(4) At very low cavitation numbers, $0<0.2$, ring interactions and jet-wall interactions destroy feedback and structuring. The new designs analyzed in this report could lead to second-generation low-sigma STRATOJETS.

The work here described should be extended to include:

(a) The effect of the presence of a wall on the ring collapse dynamics.

(b) The influence of viscosity on the cavitation inception and on bubble ring dynamics.

(c) The modeling of jet-nozzle interaction by replacing the jet shear layer either by discrete organized vortices or a very dense distribution of vortices.

(d) The continuation of the experimental work to include a thorough understanding of nozzle-jet interaction and jet behavior at low cavitation numbers.

The acquired knowledge will contribute greatly to the development of procedures for the design of an improved nozzle either for normal or low values of the cavitation number. 
Tracor Hydronautics

\section{REFERENCES}

1. Conn, A.F., Johnson, V.E., Jr., Liu, H-L., and Frederick, G.S., "Evaluation of CAVIJET ${ }^{\otimes}$ Cavitating Jets for Deep-Hole Rock Cutting," HYDRONAUTICS, Incorporated Technical Report 7821-1, August 1979 (Sandia Report No. SAND81-7067, May 1981).

2. Johnson, V.E., Jr., Lindenmuth, W.T., Conn, A.F., and Frederick, G.S., "Feasibility Study of Tuned-Resonator, Pulsating Cavitating Water Jet for Deep-Hole Drilling," HYDRONAUTICS, Incorporated Technical Report 8001-1, May 1981 (Sandia Report No. SAND81-7126, August 1981).

3. Johnson, V.E., Jr., Lindenmuth, W.T., Chahine, G.L., Conn, A.F., and Frederick, G.S., "Research and Development of Improved Cavitating Jets for Deep-Hole Drilling," Tracor Hydronautics Technical Report 8021-1, November 1982, iSandia Report No. SAND83-7461).

4. Maurer, W.C., "Advanced Drilling Techniques," Maurer Engineering, Inc. Technical Report No. TR79-1, January 1979 .

5. Wylie, E.B. and Streeter, V.L., "Fluid Transients," McGraw Hill International Book Company, New York, 1978.

6. Van Wijngaarden, L., "One-Dimensional Flow of Liquids Containing Small Gas Bubbles," Annual Review of Fluid Mechanics, 4, pp. 369-397, 1972.

7. Kinsler, L.E. and Frey, A.R., "Fundamentals of Acoustics," Ed. John wiley \& Sons, Inc., New York, 1962.

8. Crow, S.C. and Champagne, F.H., "Orderly structure in Jet Turbulence," Journal of Fluid Mechanics, 1971, Vol. 48, pp. 547-591.

9. Acton, E., "A Modelling of Large Eddies in an Axisymmetric Jet," Journal of Fluid Mechanics, 1980, Vol. 98, pp. 1-31.

10. Pocklington, H.C., "The Complete system of the Periods of a Hollow Vortex-Ring," Phil. Trans., 1895, A1, p. 603.

11. Larmor, J., "A Dynamical Theory of the Electric and Luminiferous Medium," Phil. Trans., 1894, A, p. 719.

12. Kelvin, W., Appendix to Helmholtz, Phil. Mag., 1867, Vol. 33, p. 511 .

Lamb, M., "Hydrodynamics," 6 th edition, Dover Publications, New York, pp. 239 and 245, 1945. 
Tracor Hydronautics

REFERENCES (Continued)

14. Tung, C. and Ting, L., "Motion and Decay of a Vortex in a Nonuniform Stream": Journal of Physics of Fluids, 1965, Vol. 8, pp. 1039-1051.

15. Van Dyke, M., "Perturbation Methods in Fluid Mechanics," Academic Press, 1964.

16. Batchelor, G.K., "An Introduction to Fluid Dynamics," Cambridge University Press, Cambridge, 1970.

17. Tung, C. and Ting, L., "Motion and Decay of a Vortex Ring," Journal Physics of Fluids, Vol. 10, 5, pp. 901-910, 1967.

18. Saffman, P.G., "The Velocity of Viscous Vortex Rings," Studies Applied Mathematics, Vol. 49, pp. 371-380, 1970 .

19. Moore, D.W. and Saffman, P.G., "The Motion of a Vortex Filament with Axial Flow," Phil. Trans., 272A, pp. 403-429, 1972 .

20. Johnson, V.E., Jr., Chahine, G.L., Lindenmuth, W.T., Conn, A.F., Frederick, G.S., Giacchino, G.J., Jr., "Cavitating and Structured Jets for Mechanical Bits to Increase Drilling Rate," A.S.M.E. Paper, 82-Pet-13, 1982.

21. Shutler, N.D. and Mesler, R.B., "A Photographic Study of the Dynamics and Damage Capabilities of Bubbles Collapsing Near Solid Boundaries," Dept. of Chemical and Petroleum Engineering, University of Kansas Report, April 1964.

22. Lauterborn, w., "Cavitation Bubble Dynamics - New Tools for an Intricate Problem," Applied Scientific Research, 38 : $165-179,1982$.

23. Plesset, M.S. and Prosperetti, A., "Bubble Dynamics and Cavitation," Annual Review of Journal Fluid Mechanics, 9 , pp. 145-185, 1977 .

24. Chahine, G.L., "Interaction Between an Oscillating Bubble and a Free Surface," Journal of Fluids Engineering, Trans., ASME, Vol. 99, pp. 70 $\overline{9-716,1977 . ~}$

25. Genoux, Ph.F., "Etude Asymptotique de Mouvement et des Oscillations d'un Tore de Vapeur, Modelisation d'un Jet Cavitant Oscillant," Thèse de Docteur-Ingenieur, Universite Paris VI, 1984 . 
Tracor Hydronautics

$-91-$

REFERENCES (Concluded)

26. Kibens, V., "Discrete Noise Spectrum Generated by an Acoustically Excited Jet," Paper 75-0592, AIAA Fifth Aeroacoustics Conference, March 12-14, $197 \overline{9}$

27. Fung, Y.T., Liu, C.H., and Gunzburger, M.D., "Simulation of the Pressure Near a Jet Randomly Distributed Vortex Rings," AIAA Journal 1979, Vol. 17, pp. 553-557.

28. Chahine, G.L. and Genoux, Ph., "Collapse of a Vortex Ring," ASME Cavitation and Polyphase Flow Forum, St. Louis, Missouri, June 1982, pp. 37-41.

29. Genoux, Ph. and Chahine, G.L., "Equilibre statique et Dynamique d'un Tore de Vapeur Tourbillonnaire," Journal de Mécanique, 1983.

30. Jorqensen, D.W., "Noise from Cavitating Submerqed Jets," Journal of the Acoustical Society of America, Vol. 33, No. $10,1334-1338,1961$. 


\section{Tracor Hydronautics}

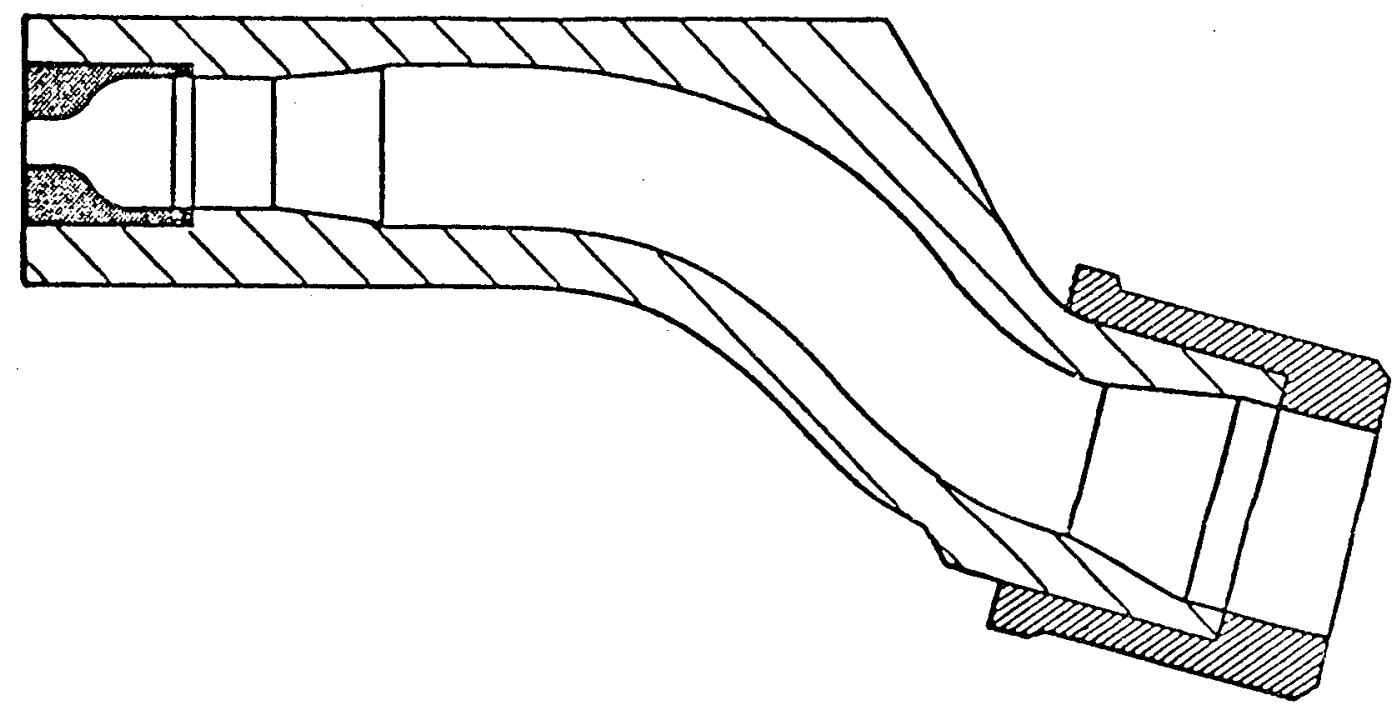

FIGURE 1 - TYPICAL EXTENDED NOZZLE FEED-TUBE FOR THREE-CONE ROLLER BIT 


\section{Tracor Hydronautics}

A. No Mean Flow (see Ref. 7)

\begin{tabular}{|l|c}
\hline Rigid cap & $z_{e}=\infty$ \\
Unflanged pipe & $z_{e}=\frac{\rho c}{A}\left(\frac{k^{2} a^{2}}{4}+0.6 j k a\right)$ \\
Infinite flange & $z_{e}=\frac{\rho c}{A}\left(\frac{k^{2} a^{2}}{2}+j \frac{8}{3} \frac{k a}{\pi}\right)$ \\
Orifice & $z_{e}=\frac{\rho c}{A}\left(\frac{k^{2} a_{0}^{2}}{2}+j k \ell^{\prime}\right)$
\end{tabular}
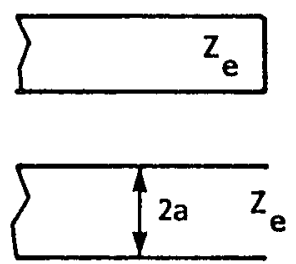

$A=\pi a^{2}$
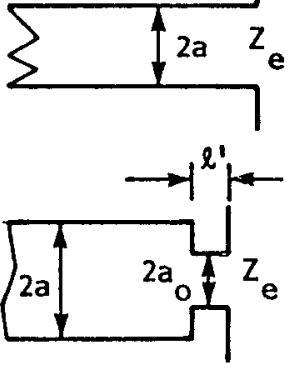

B. With Mean Flow (see Ref. 5)

Open end or constant pressure head

Closed end or fixed discharge

Oscillating valve

Fixed Orifice

$$
z_{e}=0
$$$$
z_{e}=\infty
$$$$
Z_{e}=\frac{2 \Delta \mathrm{P}_{0}}{A \bar{V}_{0}}\left(1-\frac{T_{v}}{\tau}\right)
$$

$\mathrm{T}_{\mathrm{v}}$ total closure,

$\tau$ position parameter

$$
\mathrm{z}_{\mathrm{e}}=\frac{2 \Delta \mathrm{p}_{\mathrm{o}}}{\mathrm{A} \overline{\mathrm{V}}_{\mathrm{o}}}
$$
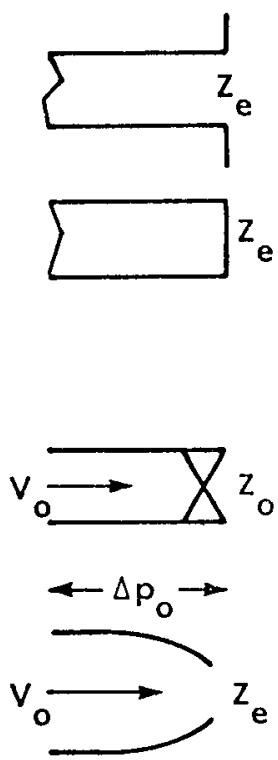

FIGURE 2 - SOME TYPICAL END IMPEDANCES 


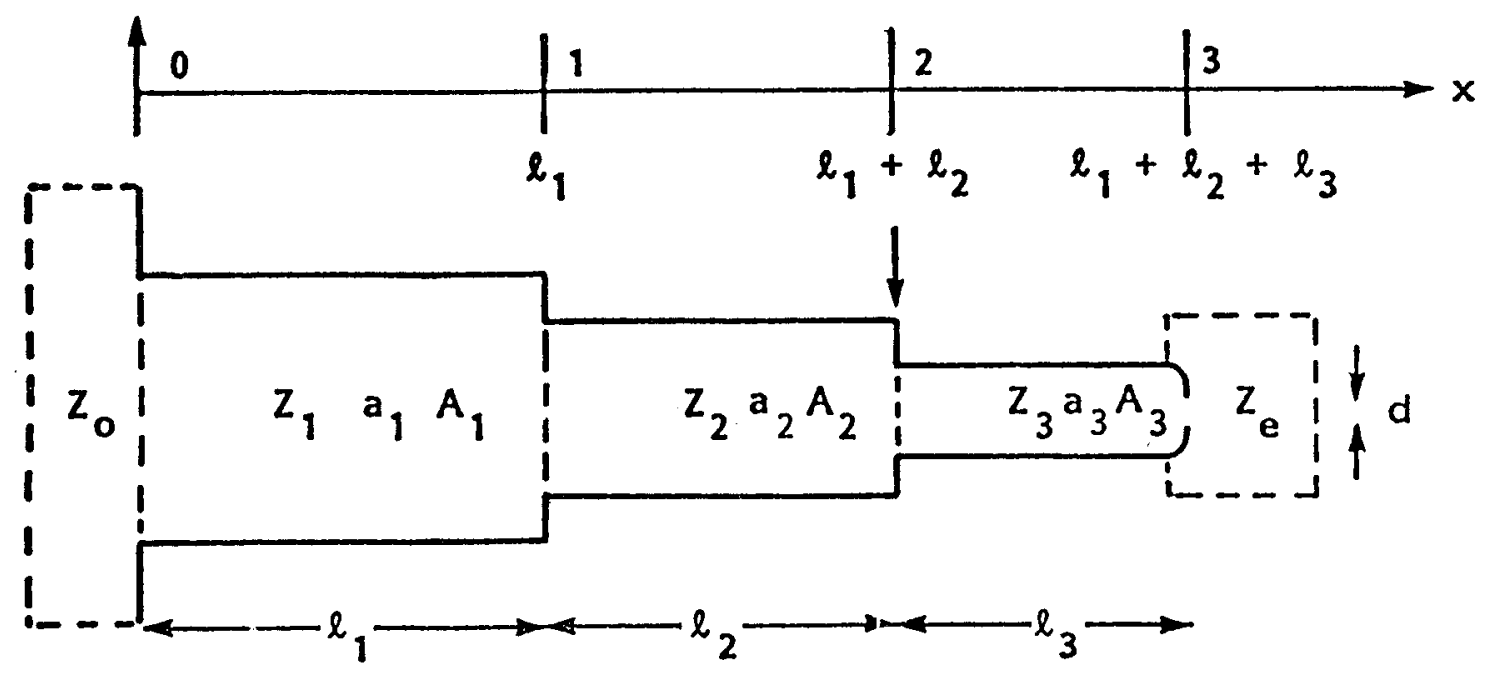

FIGURE 3 - ORGAN PIPE WITH SERIES OF DIFFERENT CROSS SECTIONS

(pipe area: $A_{i}$; pipe radius: $a_{i}$ 
Tracor Hydronautics

$-95-$

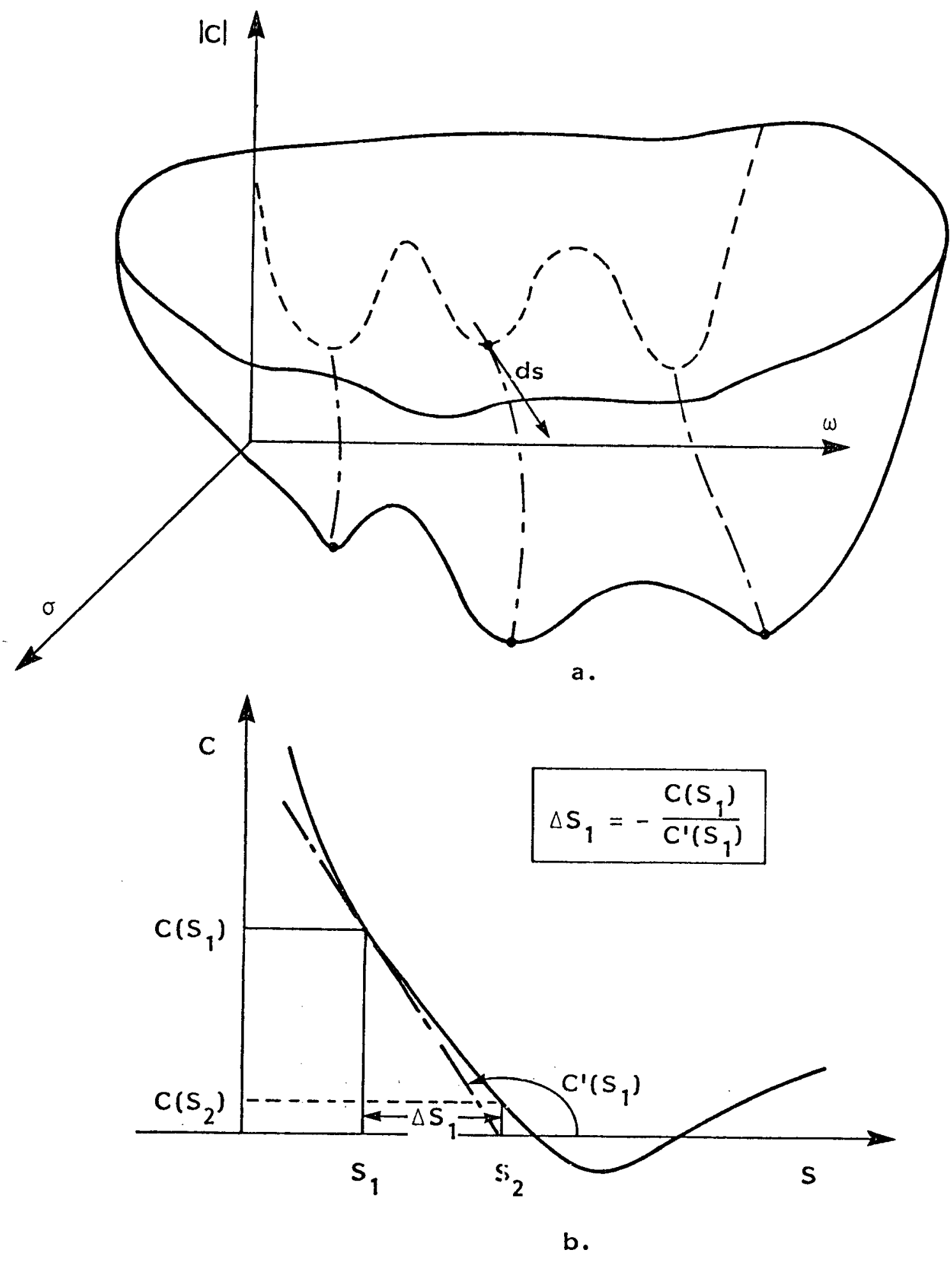

FIGURE $4 a, b$ - SCHEMATICS OF DETERMINATION OF THE ZEROS OF THE COMPLEX FUNCTION C (EQUATION (42)) 

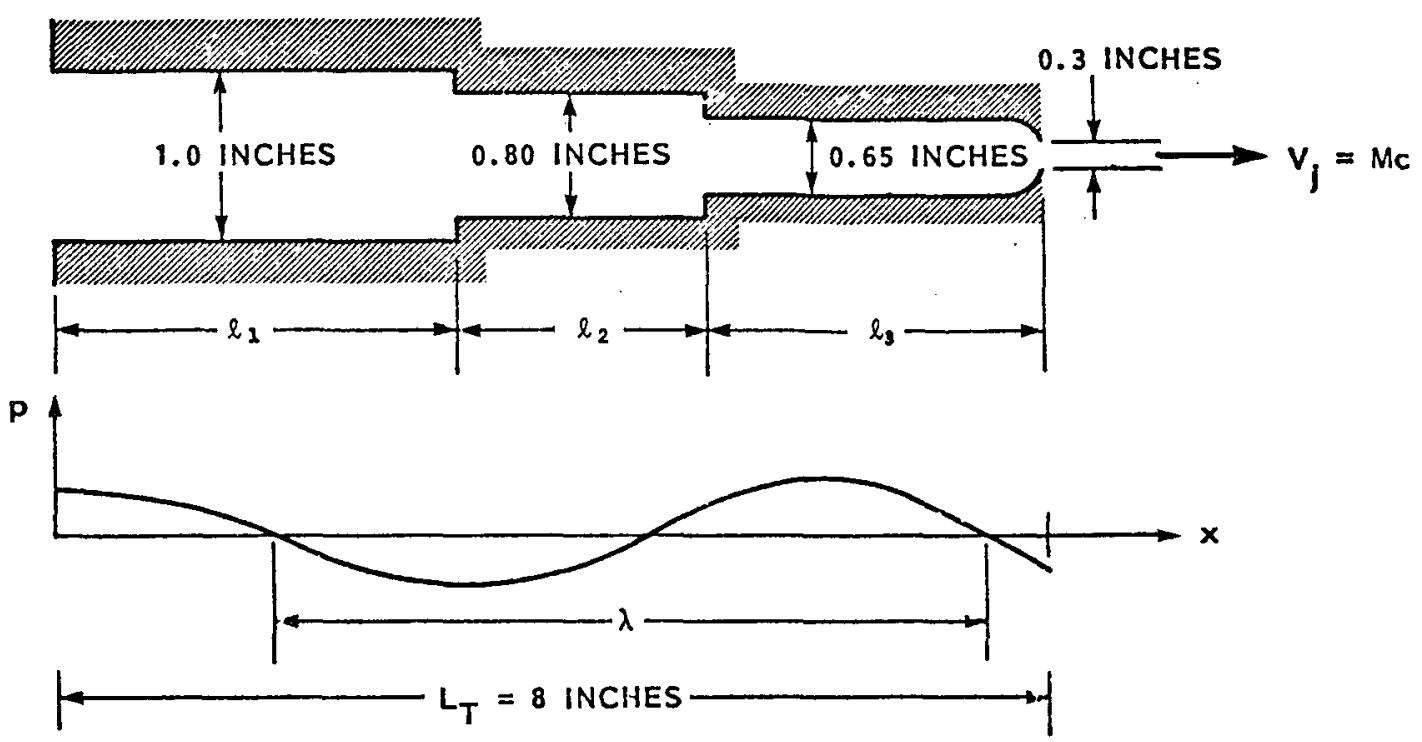

\begin{tabular}{|c|c|c|c|c|c|c|c|c|c|c|c|c|c|c|c|}
\hline \multicolumn{2}{|c|}{$\ell_{3}=8$} & \multicolumn{2}{|c|}{$\ell_{2}=2, \ell_{3}=6$} & \multicolumn{2}{|c|}{$\ell_{2}=2.6, \ell_{3}=5.4$} & \multicolumn{2}{|c|}{$\ell_{2}=3.2, \ell_{2}=4.8$} & \multicolumn{2}{|c|}{$\ell_{2}=4, \ell_{3}=4$} & \multicolumn{2}{|c|}{$\ell_{2}=4.8, \ell 3=3.2$} & \multicolumn{2}{|c|}{$\ell 2=6, \ell_{3}=2$} & \multicolumn{2}{|c|}{$\ell_{1}=\ell_{2}=\ell_{3}=8 / 3$} \\
\hline$\omega$ & $\sigma$ & $\omega$ & $\pi$ & $\omega$ & $\sigma$ & $\omega$ & $\sigma$ & $\omega$ & $\sigma$ & $\omega$ & $\sigma$ & $\omega$ & $\sigma$ & $\omega$ & $\sigma$ \\
\hline 3.142 & -0.229 & 2.511 & -0.174 & 2.250 & -0.146 & 1.961 & -0.119 & - & - & 1.208 & -0.075 & 0.731 & -0.055 & 1.063 & -0.059 \\
\hline 6.283 & -0.229 & - & - & 4.123 & -0.143 & 3.652 & -0.145 & 3.142 & -0.138 & 2.596 & -0.100 & - & - & 2.078 & -0.059 \\
\hline 9.425 & -0.229 & 6.914 & -0.174 & 6.341 & -0.174 & 5.773 & -0.145 & - & - & 3.687 & -0.100 & 2.410 & -0.055 & 3.142 & -0.110 \\
\hline 12.566 & -0.229 & 9.425 & -0.188 & 8.619 & -0.149 & 7.464 & -0.119 & 6.283 & -0.138 & 5.075 & -0.075 & 3.142 & -0.077 & 4.205 & -0.059 \\
\hline 15.708 & -0.229 & 11.936 & -0.174 & 10.498 & -0.140 & 9.425 & -0.159 & - & - & 6.283 & -0.118 & 3.873 & -0.055 & 5.220 & -0.059 \\
\hline 18.849 & -0.229 & - & - & 12.683 & -0.173 & 11.386 & -0.119 & 9.425 & -0.138 & 7.492 & -0.075 & - & - & 6.283 & -0.110 \\
\hline
\end{tabular}

Figure 5 - Theoretical Values of Natural Frequencies, $\omega$ and Attenuation Factor, $\sigma . \quad M=0.05$ 

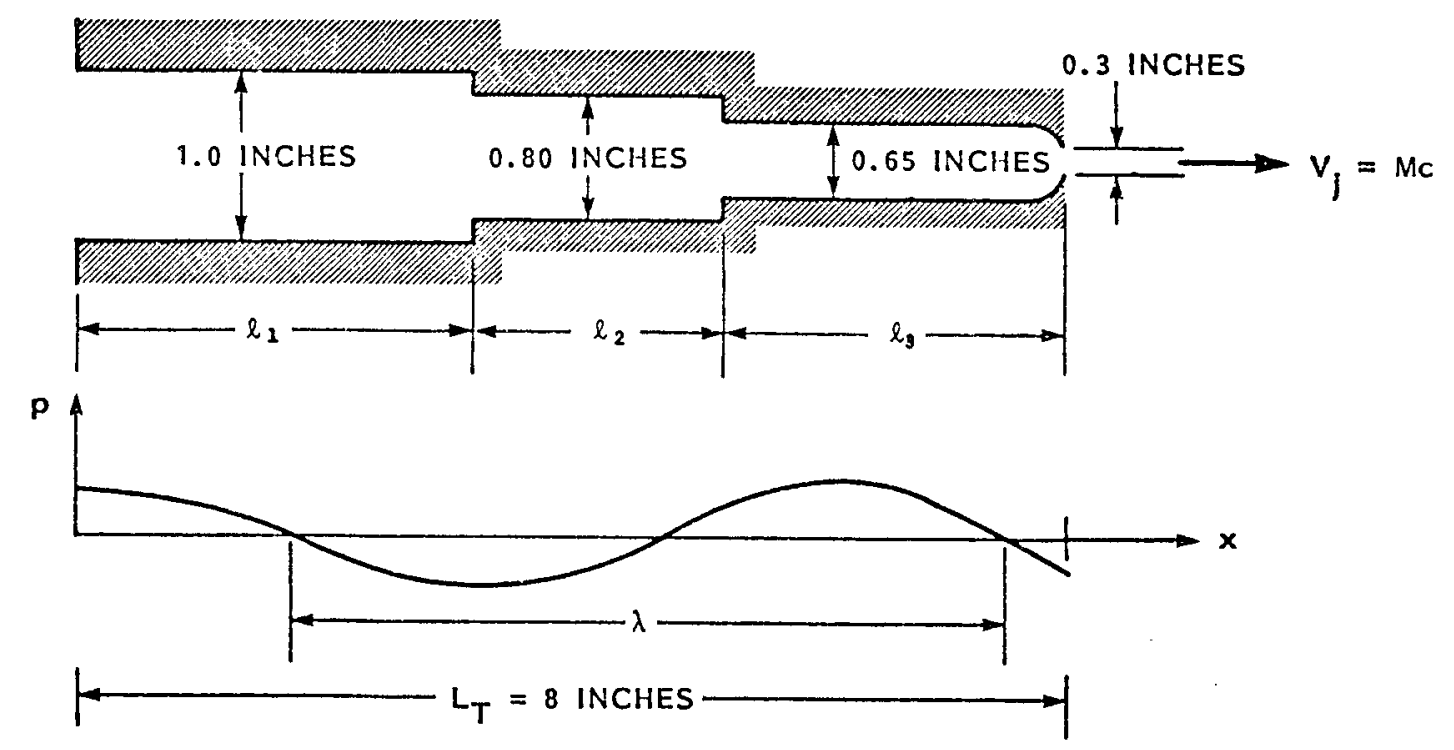

\begin{tabular}{|c|c|c|c|c|c|c|c|c|c|c|c|c|c|c|c|}
\hline \multicolumn{2}{|c|}{$\ell_{3}=8$} & \multicolumn{2}{|c|}{$\ell_{2}=2, \ell_{3}=6$} & \multicolumn{2}{|c|}{$\ell_{2}=2.6, \ell_{3}=5.4$} & \multicolumn{2}{|c|}{$\ell_{2}=3.2, \ell_{2}=4.8$} & \multicolumn{2}{|c|}{$\ell_{2}=4, \ell_{3}=4$} & \multicolumn{2}{|c|}{$\ell_{2}=4.8, \ell 3=3.2$} & \multicolumn{2}{|c|}{$\ell 2=6, \ell_{3}=2$} & \multicolumn{2}{|c|}{$\ell_{1}=\ell_{2}=\ell_{3}=8 / 3$} \\
\hline$\omega$ & $\sigma$ & $\omega$ & $\sigma$ & $\omega$ & $\sigma$ & $\omega$ & $\sigma$ & $\omega$ & $\sigma$ & $\omega$ & $\sigma$ & $\omega$ & $\sigma$ & $\omega$ & $\sigma$ \\
\hline 3.141 & -0.485 & 2.514 & $-0 . .367$ & 2.255 & -0.309 & 1.966 & -0.250 & - & - & 1.203 & -0.154 & 0.720 & -0.116 & - & - \\
\hline 6.283 & -0.485 & - & - & 4.117 & -0.302 & 3.645 & -0.308 & 3.142 & -0.293 & 2.607 & -0.207 & - & - & - & - \\
\hline 9.425 & -0.485 & 6.910 & -0.368 & 6.341 & -0.368 & 5.779 & -0.308 & - & - & 3.676 & -0.208 & 2.421 & -0.116 & 3.142 & -0.236 \\
\hline 12.566 & -0.485 & 9.425 & -0.397 & 8.624 & -0.316 & 7.459 & -0.250 & 6.283 & -0.293 & 5.080 & -0.154 & 3.142 & -0.167 & - & - \\
\hline 15.708 & -0.485 & 11.939 & -0.367 & 10.494 & -0.296 & 9.425 & -0.337 & - & - & 6.283 & -0.246 & 3.862 & -0.116 & - & - \\
\hline 18.849 & -0.485 & - & - & 12.682 & -0.367 & 11.391 & -0.250 & 9.425 & -0.293 & 7.486 & -0.154 & - & - & 6.283 & -0.236 \\
\hline
\end{tabular}

Figure 6 - Theoretical Values of Natural Frequencies, w and Attenuation Factor, 0. M=0. 1 


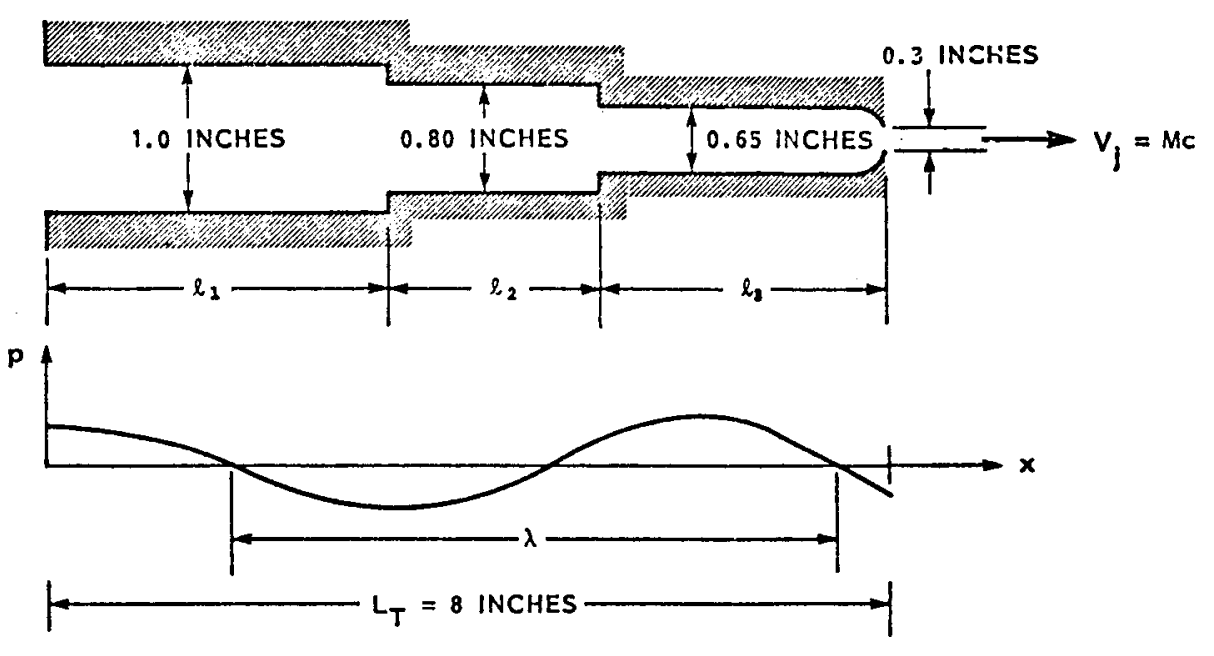

\begin{tabular}{|c|c|c|c|c|c|c|c|c|c|c|c|c|c|c|c|}
\hline \multicolumn{2}{|c|}{$\ell_{3}=8$} & \multicolumn{2}{|c|}{$\ell_{2}=2, \ell_{3}=6$} & \multicolumn{2}{|c|}{$\ell_{2}=2.6, \ell_{3}=5.4$} & \multicolumn{2}{|c|}{$\ell_{2}=3.2, \ell_{3}=4.8$} & \multicolumn{2}{|c|}{$\ell_{2}=4, \ell_{3}=4$} & \multicolumn{2}{|c|}{$\ell_{2}=4.8, \ell_{3}=3.2$} & \multicolumn{2}{|c|}{$\ell_{2}=6, \ell_{3}=2$} & \multicolumn{2}{|c|}{$\ell_{1}=\ell_{2}=\ell_{3}=8 / 3$} \\
\hline $\mathrm{L}_{\mathrm{T}} / \lambda$ & $\beta_{0}$ & $\mathrm{~L}_{\mathrm{T}} / \lambda$ & $\beta_{0}$ & $\mathrm{~L}_{\mathrm{T}} / \lambda$ & $B_{0}$ & $\mathrm{~L}_{\mathrm{T}} / \lambda$ & $\beta_{0}$ & $\mathrm{~L}_{\mathrm{T}} / \lambda$ & $\beta_{0}$ & $\mathrm{~L}_{\mathrm{T}} / \lambda$ & $B_{0}$ & $\mathrm{~L}_{\mathrm{T}} / \lambda$ & $\beta_{0}$ & $\mathrm{~L}_{\mathrm{T}} / \lambda$ & $B_{0}$ \\
\hline 0.50 & 1.00 & 0.53 & 0.99 & 0.53 & 1.06 & 0.52 & 1.15 & - & - & 0.48 & 1.22 & 0.46 & 1.03 & 0.51 & 1.29 \\
\hline 1.00 & 1.00 & - & - & 0.97 & 1.08 & 0.97 & 0.94 & 1.00 & 0.83 & 1.03 & 0.91 & - & - & 0.99 & 1.28 \\
\hline 1.50 & 1.00 & 1.47 & 0.99 & 1.49 & 0.89 & 1.53 & 0.94 & - & - & 1.47 & 0.91 & 1.54 & 1.03 & 1.50 & 0.69 \\
\hline 2.00 & 1.00 & 2.00 & 0.92 & 2.03 & 1.03 & 1.98 & 1.15 & 2.00 & 0.83 & 2.02 & 1.22 & 2.00 & 0.74 & 2.01 & 1.28 \\
\hline 2.50 & 1.00 & 2.53 & 0.99 & 2.48 & 1.10 & 2.50 & 0.86 & - & - & 2.50 & 0.77 & 2.46 & 1.03 & 2.49 & 1.28 \\
\hline 3.00 & 1.00 & - & - & 2.99 & 0.89 & 3.02 & 1.16 & 3.00 & 0.83 & 2.98 & 1.22 & - & - & 3.00 & 0.69 \\
\hline
\end{tabular}

FIGURE 7 - THEORETICAL VALUES OF WAVELENGTHS, $\lambda / L_{T}$, AND AMPLIFICATION GAINS, $\beta_{0}$. MACH NUMBER $=0.05$ 


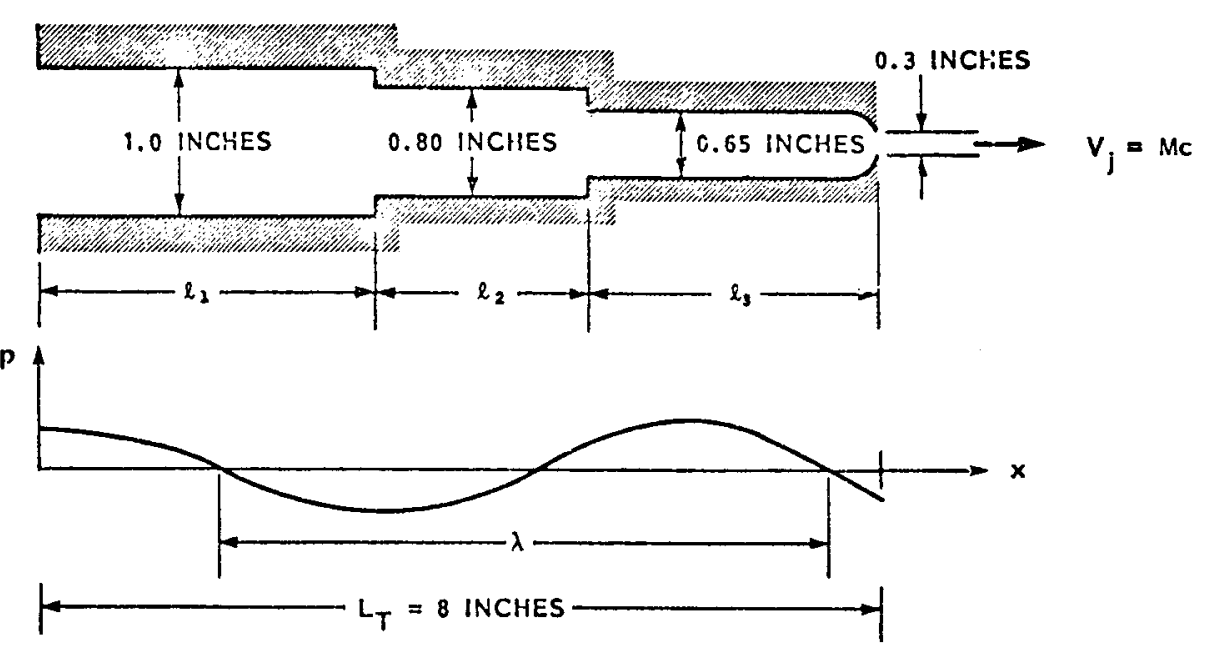

\begin{tabular}{|c|c|c|c|c|c|c|c|c|c|c|c|c|c|c|c|}
\hline \multicolumn{2}{|c|}{$l_{3}=8$} & \multicolumn{2}{|c|}{$l_{2}=2, l_{3}=6$} & \multicolumn{2}{|c|}{$\ell_{2}=2.6, \ell_{3}=5.4$} & \multicolumn{2}{|c|}{$\ell_{2}=3.2, \ell_{3}=4.8$} & \multicolumn{2}{|c|}{$\ell_{2}=4, \ell_{3}=4$} & \multicolumn{2}{|c|}{$\ell_{2}=4.8, \ell_{3}=3.2$} & \multicolumn{2}{|c|}{$l_{2}=6, l_{3}=2$} & \multicolumn{2}{|c|}{$l_{1}=l_{2}=l_{3}=8 / 3$} \\
\hline $\mathrm{L}_{\mathrm{T}} / \lambda$ & $\beta_{0}$ & $\mathrm{~L}_{\mathrm{T}} / \lambda$ & $\beta_{0}$ & $\mathrm{~L}_{\mathrm{T}} / \lambda$ & ${ }_{0}$ & $\mathrm{~L}_{\mathrm{T}} / \lambda$ & $\beta_{0}$ & $\mathrm{~L}_{\mathrm{T}} / \lambda$ & $B_{0}$ & $\mathrm{~L}_{\mathrm{T}} / \lambda$ & $B_{0}$ & $\mathrm{~L}_{\mathrm{T}} / \lambda$ & $\beta_{0}$ & $\mathrm{~L}_{\mathrm{T}} / \lambda$ & $\beta_{0}$ \\
\hline 0.50 & 1.00 & 0.53 & 0.99 & 0.53 & 1.05 & 0.52 & 1.16 & - & - & 0.48 & 1.26 & 0.46 & 1.04 & - & - \\
\hline 1.00 & 1.00 & - & - & 0.97 & 1.08 & 0.97 & 0.94 & 1.00 & 0.82 & 1.04 & 0.93 & - & - & - & - \\
\hline 1.50 & 1.00 & 1.47 & 0.98 & 1.49 & 0.88 & 1.53 & 0.94 & - & - & 1.46 & 0.93 & 1.54 & 1.04 & 1.50 & 0.68 \\
\hline 2.00 & 1.00 & 2.00 & 0.91 & 2.03 & 1.03 & 1.98 & 1.16 & 2.00 & 0.82 & 2.02 & 1.26 & 2.00 & 0.72 & - & - \\
\hline 2.50 & 1.00 & 2.53 & 0.97 & 2.47 & 1.10 & 2.50 & 0.86 & - & - & 2.50 & 0.78 & 2.46 & 1.04 & - & - \\
\hline 3.00 & 1.00 & - & - & 2.99 & 0.89 & 3.02 & 1.16 & 3.00 & 0.82 & 2.98 & 1.26 & - & - & 3.00 & 0.68 \\
\hline
\end{tabular}

FIGURE 8 - THECRETICAL VALUES OF WAVELENGTHS, $\lambda / L_{T}$, AND AMPLIFICATION GAINS, $\beta_{0}$, MACH NUMBER $=0.1$ 


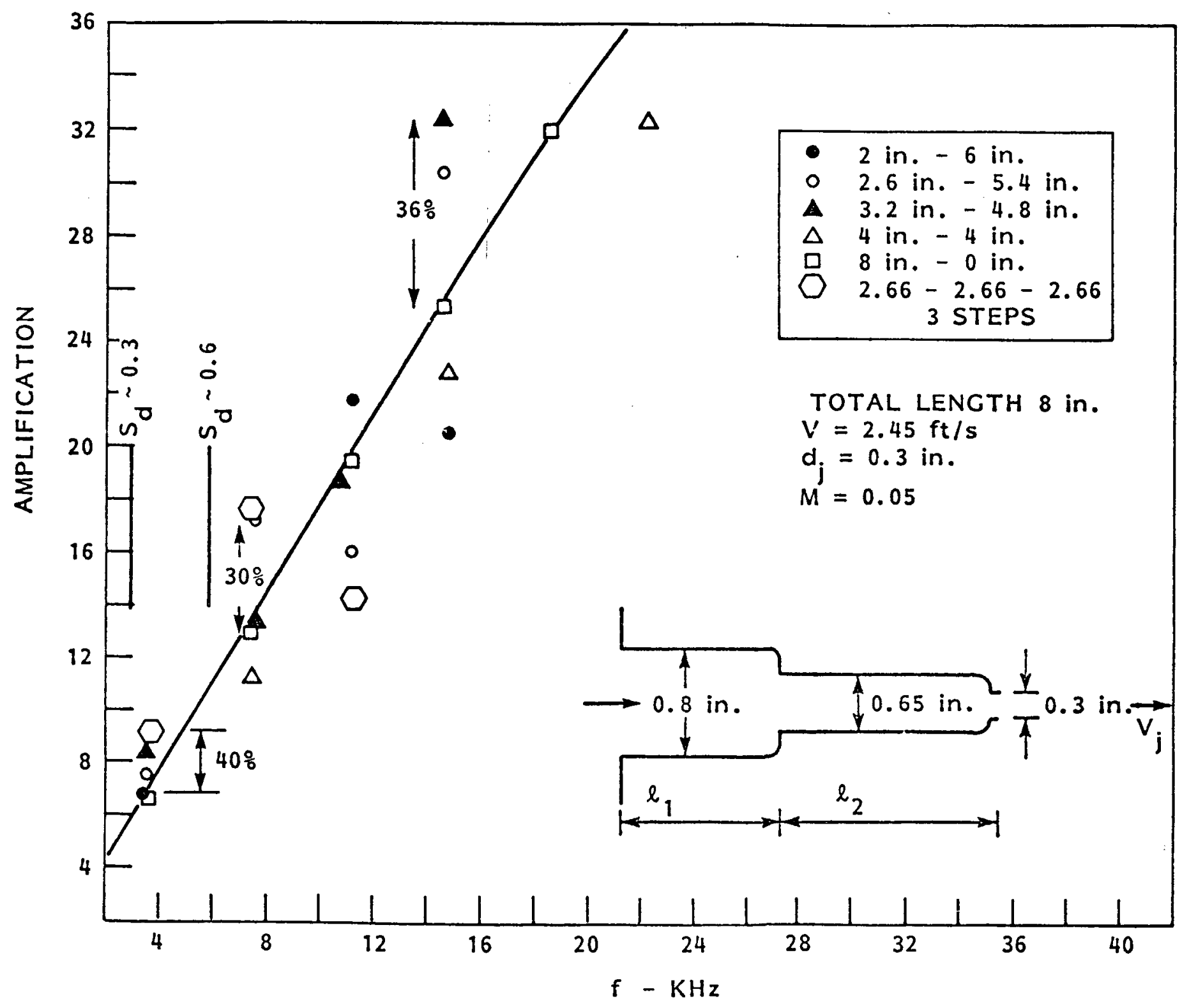

곸 


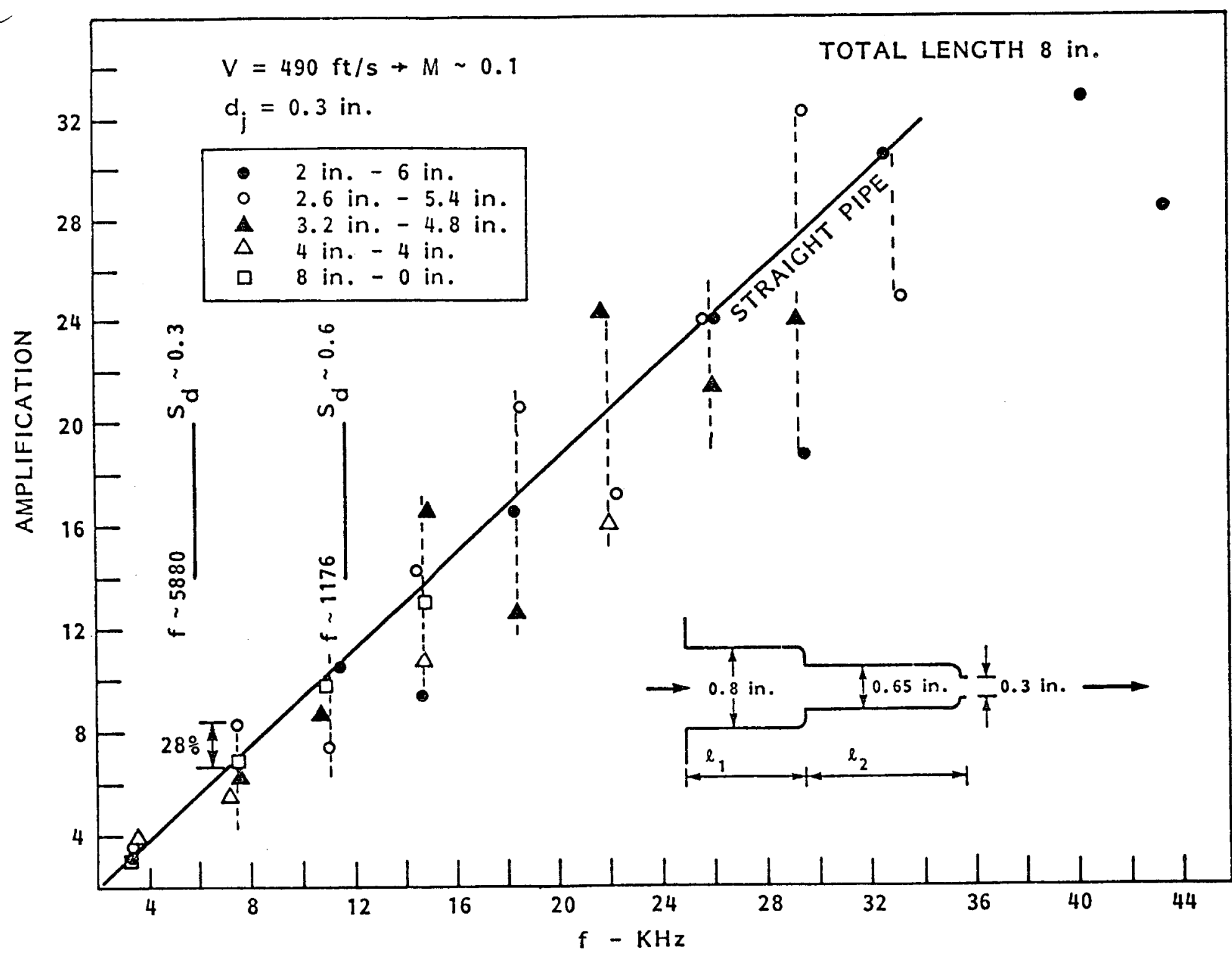

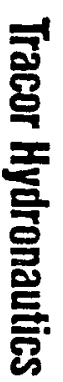

FIGURE 10 - AMPLIFICATION VERSUS NATURAL FREQUENCIES $M=0.10$ 


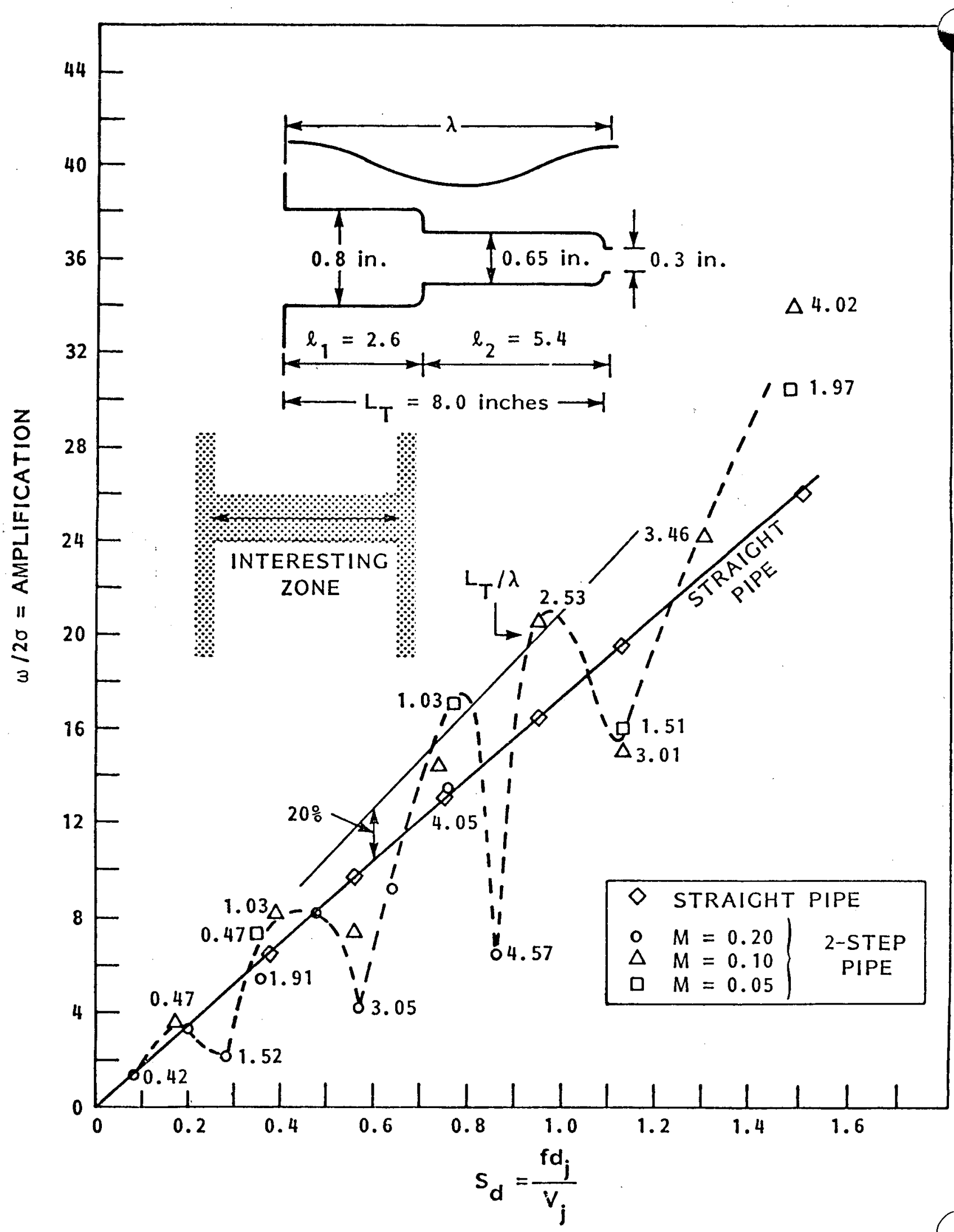

FIGURE 11 - ACOUSTICAL AMPLIFICATION VERSUS STROUHAL NUMBER 


\section{Tracor Hydronautics}
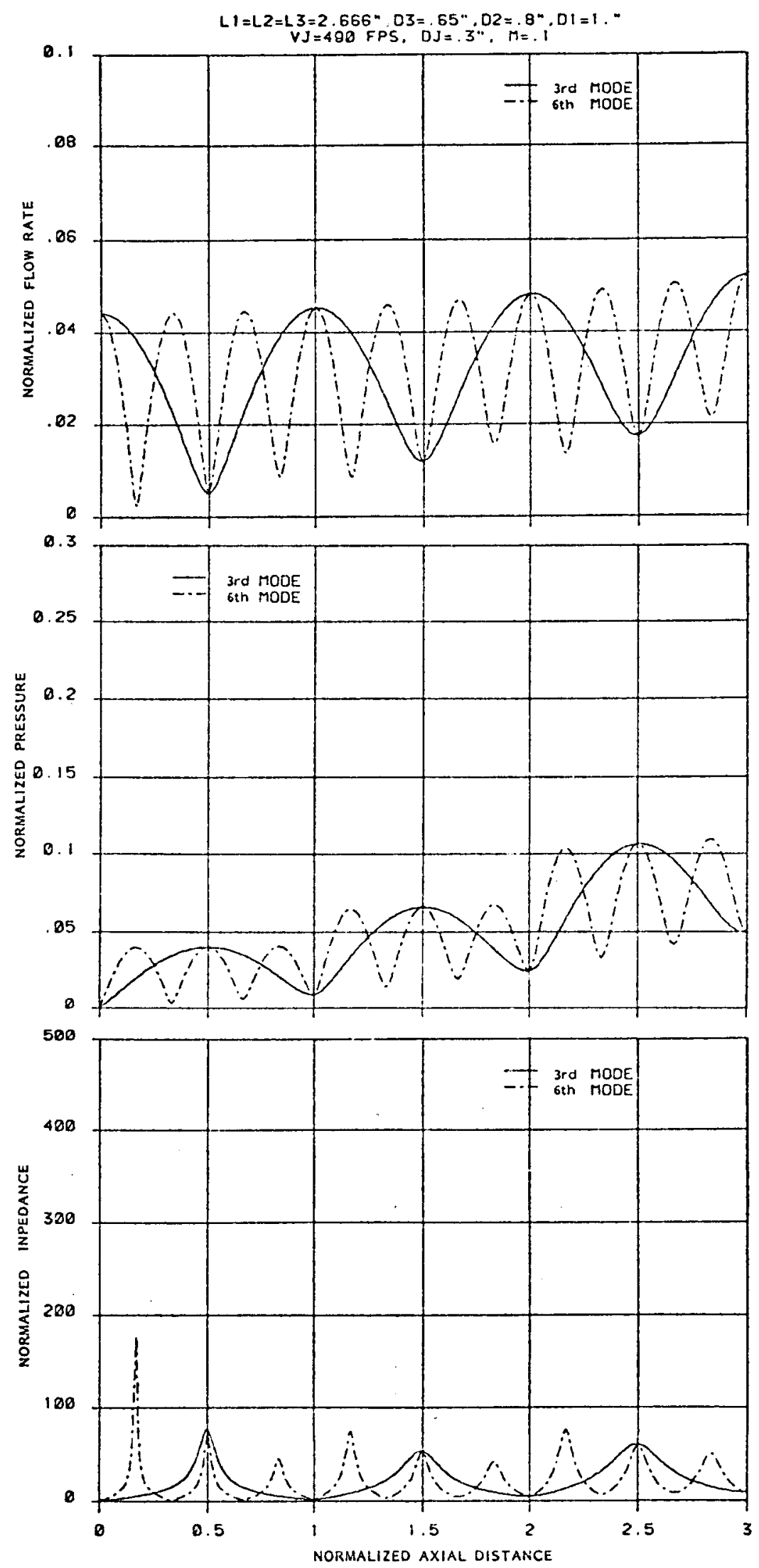

FIGURE 12 - NORMALIZED FLUCTUATING FLOW RATE, PRESSURE AND IMPEDANCE VERSUS THE AXIAL DISTANCE ALONG A THREE-STEPPED TUBE, 3rd, 6th MODE, $M=0.1$ 
Tracor Hydronautics
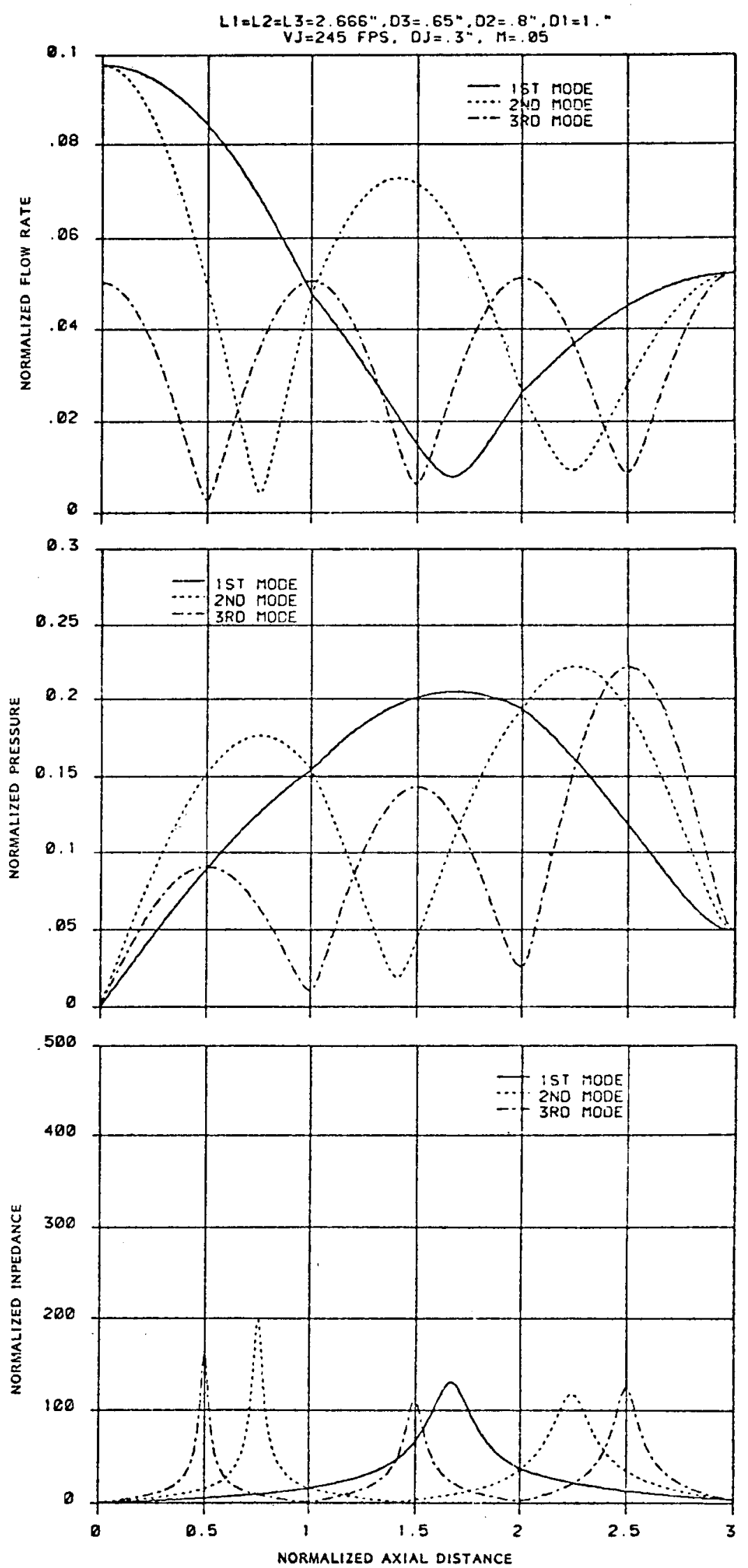

FICURE 13 - NORMALIZED FLUCTUATING FLOW RATE, PRESSURE AND IMPEDANCE VERSUS THE AXIAL DISTANCE ALONC A THREE-STEPPED TUBE, 1st, 2nd, 3rd, MODE, $M=0.05$ 

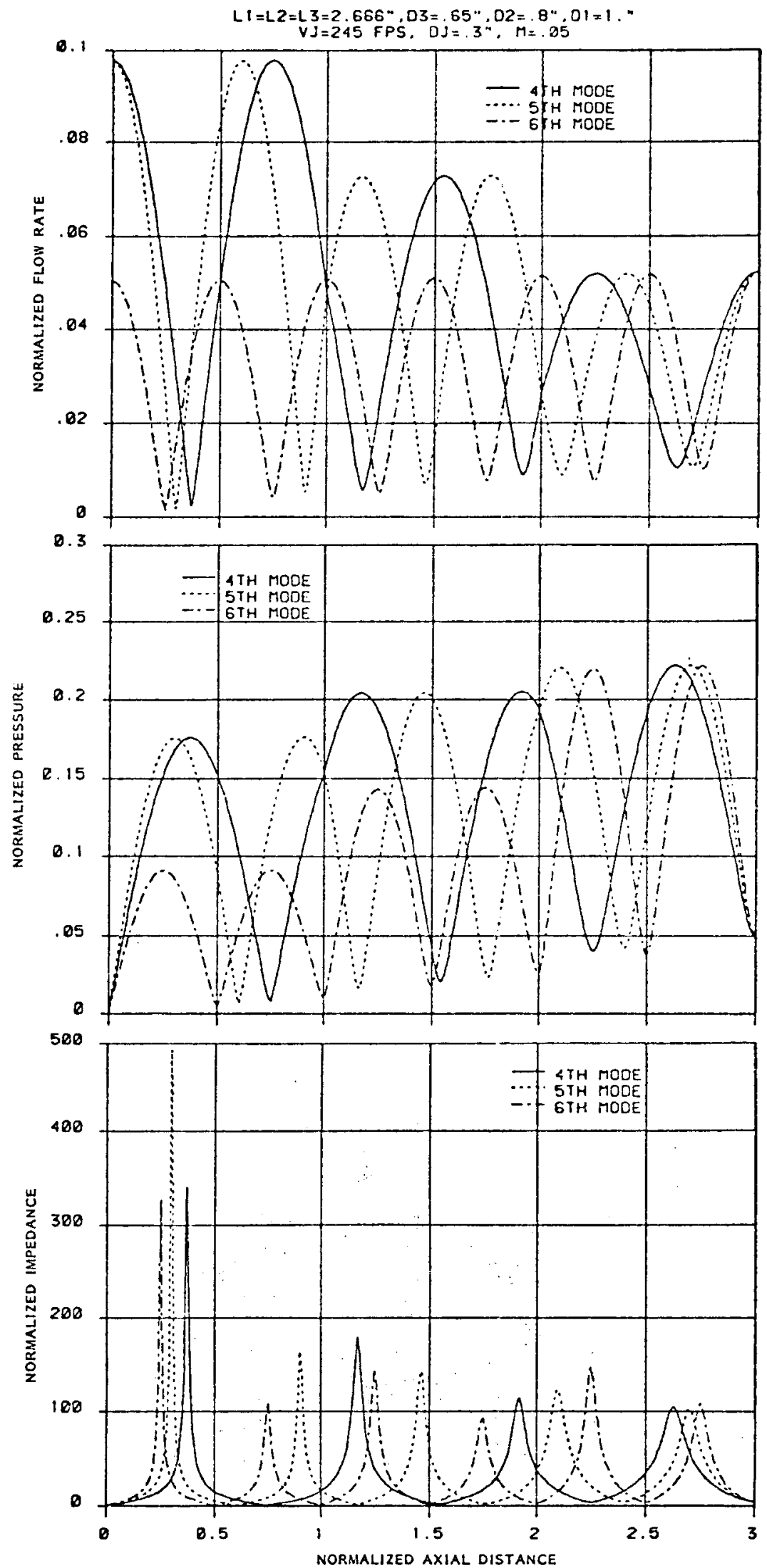

FIGURE 14 - NORMALIZED FLUCTUATING FLOW RATE, PRESSURE AND IMPEDANCE VERSUS THE AXIAL DISTANCE ALONG A THREE-STEPPED TUBE, 4th, 5th, 6th MODE, $M=0.05$ 


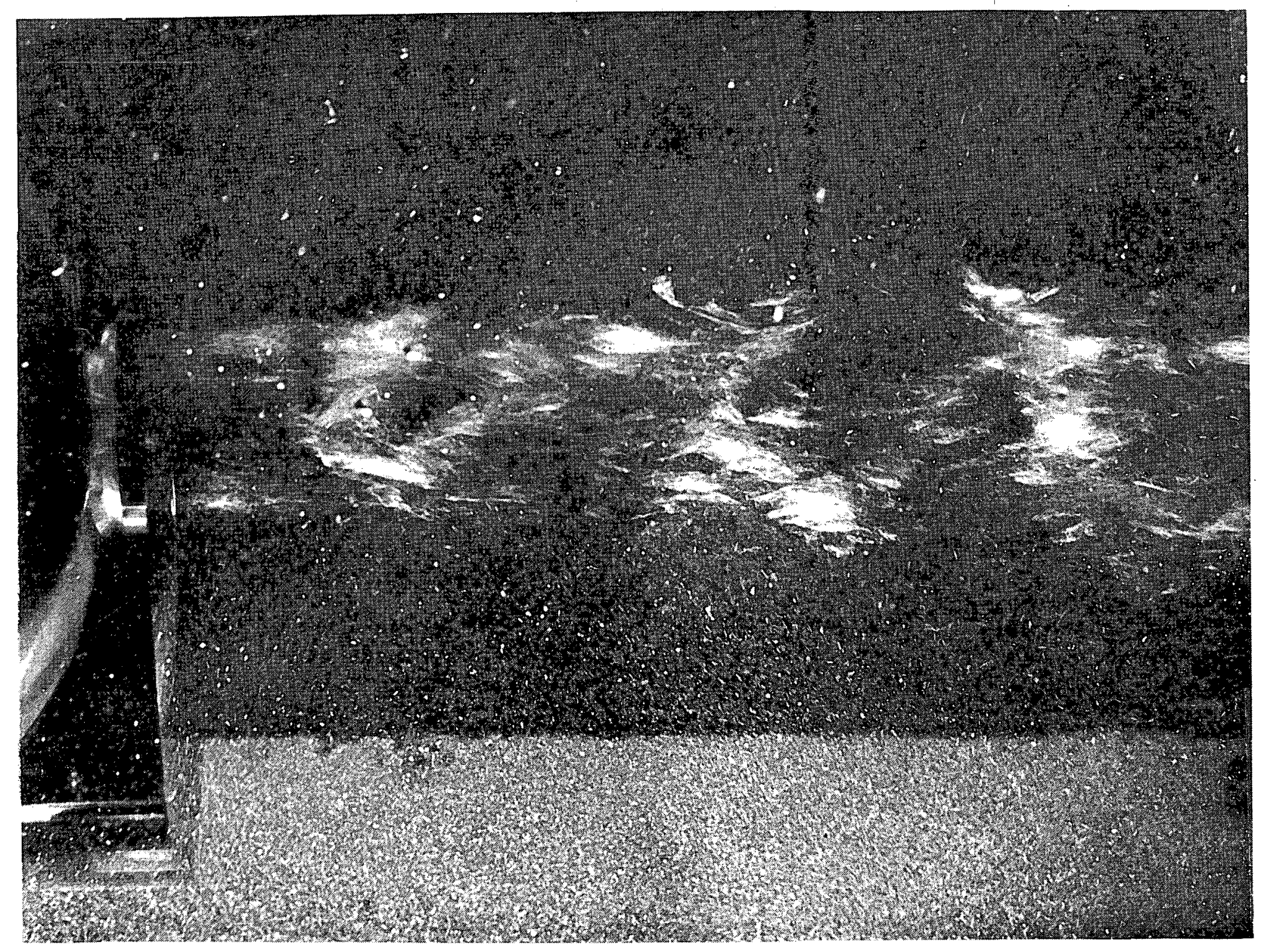

콩

FIGURE 15 - CAVITATION AS OBSERVED BY SHORT DURATION EXPOSURE, SHOWING JET STRUCTURING; photograph

0 taken in High Speed Channel 


\section{Tracor Hydronautics}

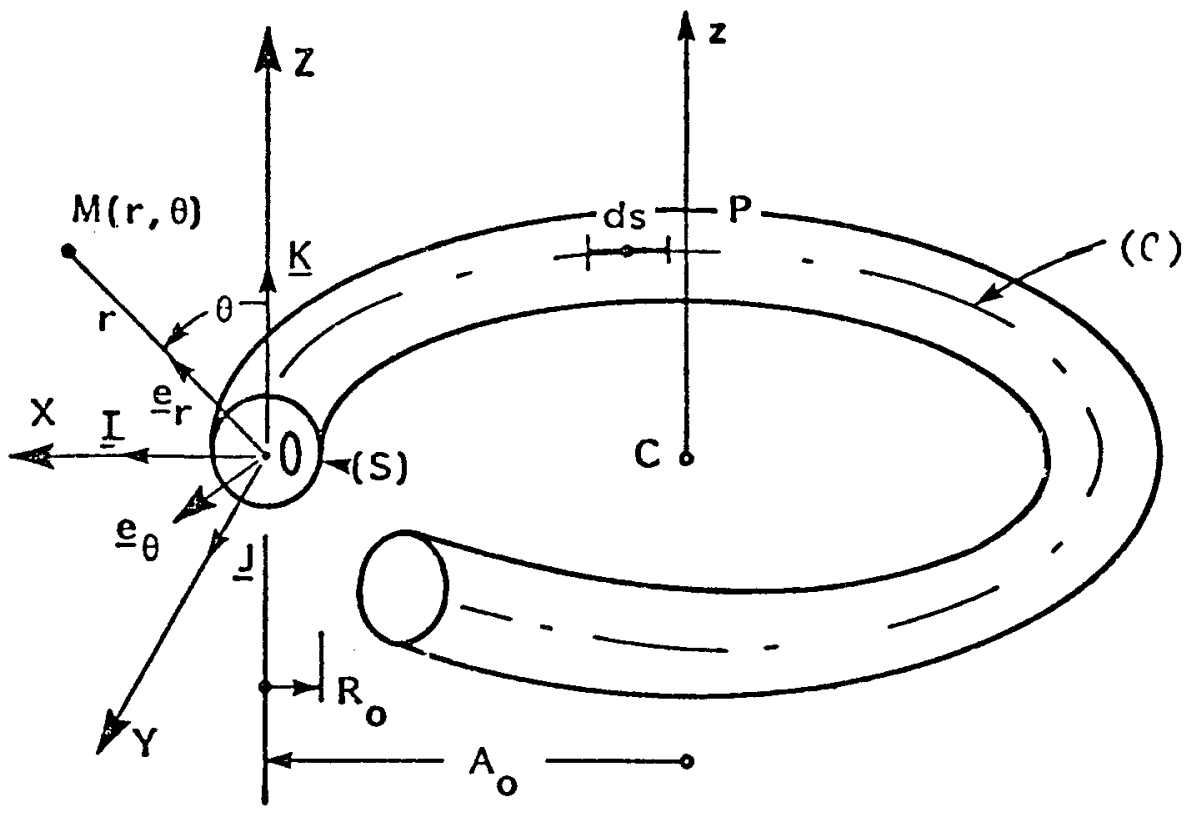

FIGURE 16 - DEFINITION OF COORDINATE SYSTEM 


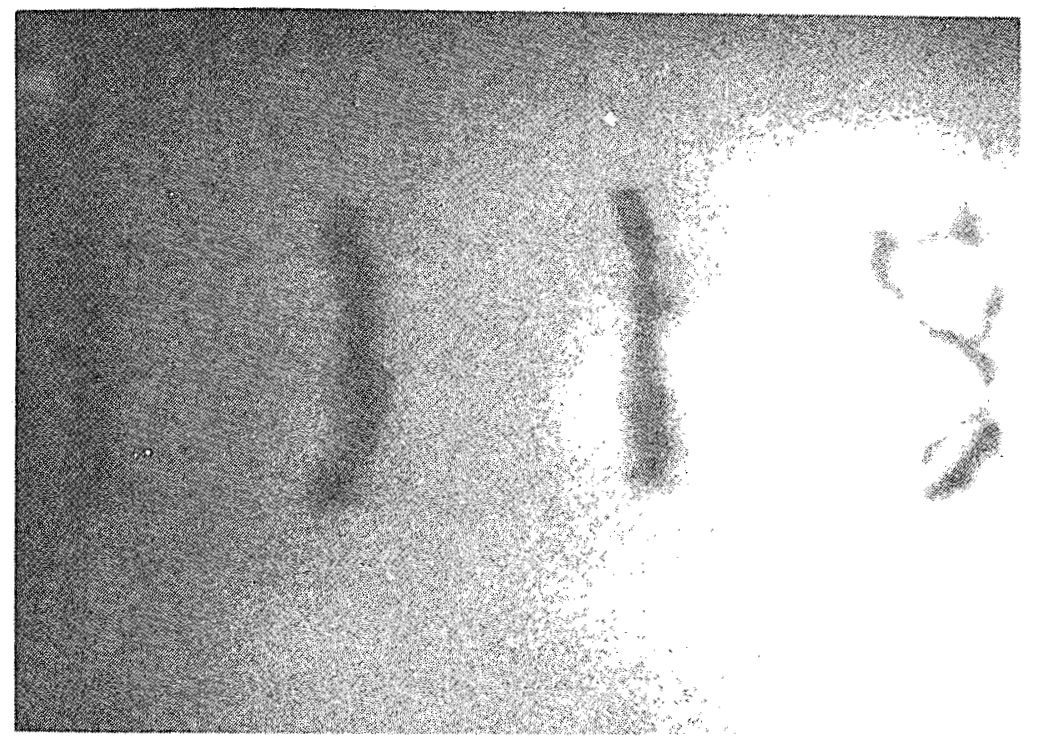

CAVITATING VORTEX RINGS AROUND A SERVOJET. $V \simeq 125 \mathrm{~m} / \mathrm{s}, \sigma \simeq 0.94, f \simeq 14 \mathrm{kHz}, S_{d} \approx 0.59$
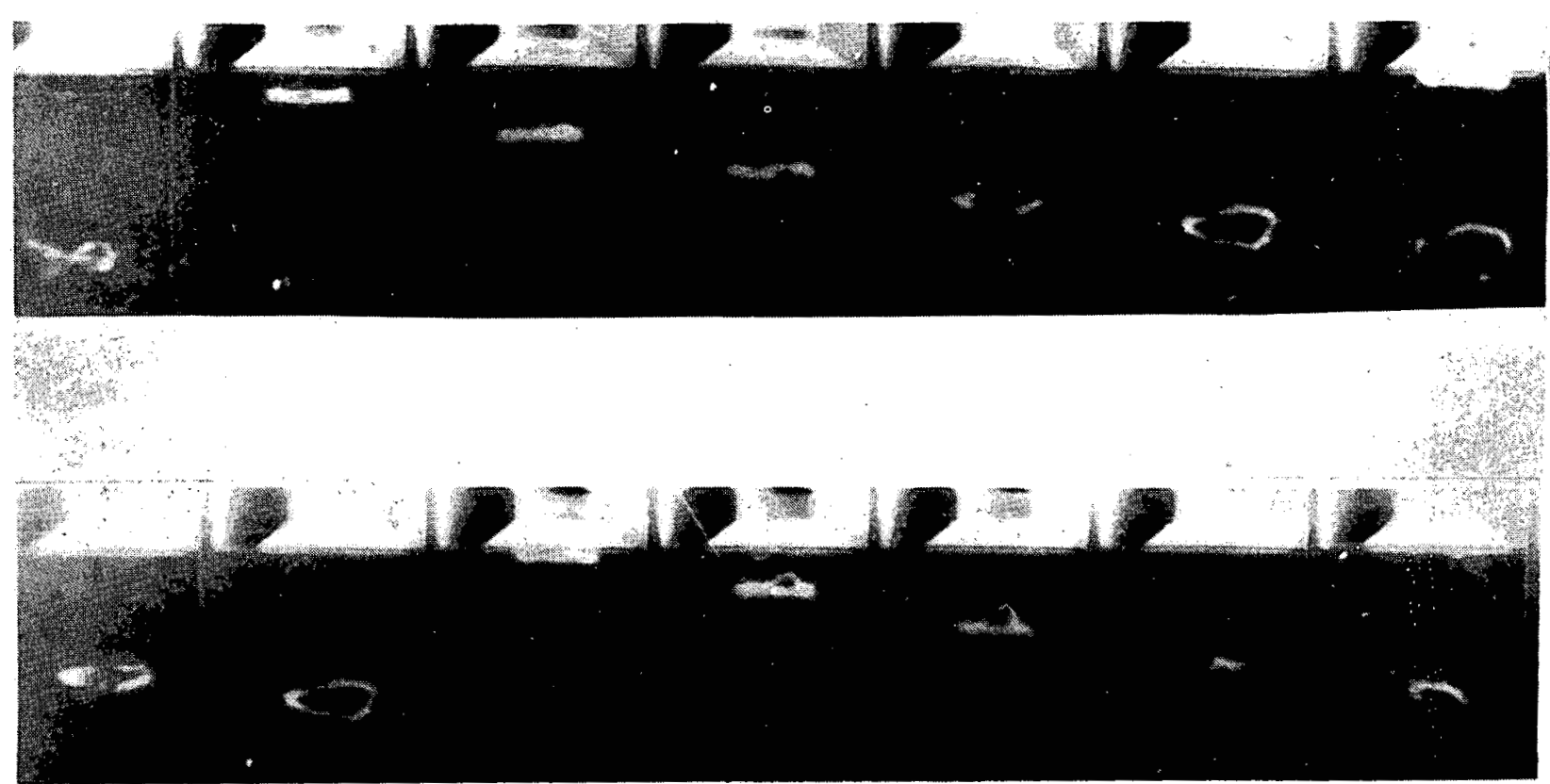

FIGURE 17 - HIGH SPEED PHOTOGRAPHY OF CAVITATING RINGS EMITTED

FROM A SERVOJET. $\triangle \mathrm{P}=60 \mathrm{psi}, \mathrm{P}_{\mathrm{a}}=43 \mathrm{psi}, \mathrm{f}=700 \mathrm{~Hz}$ 


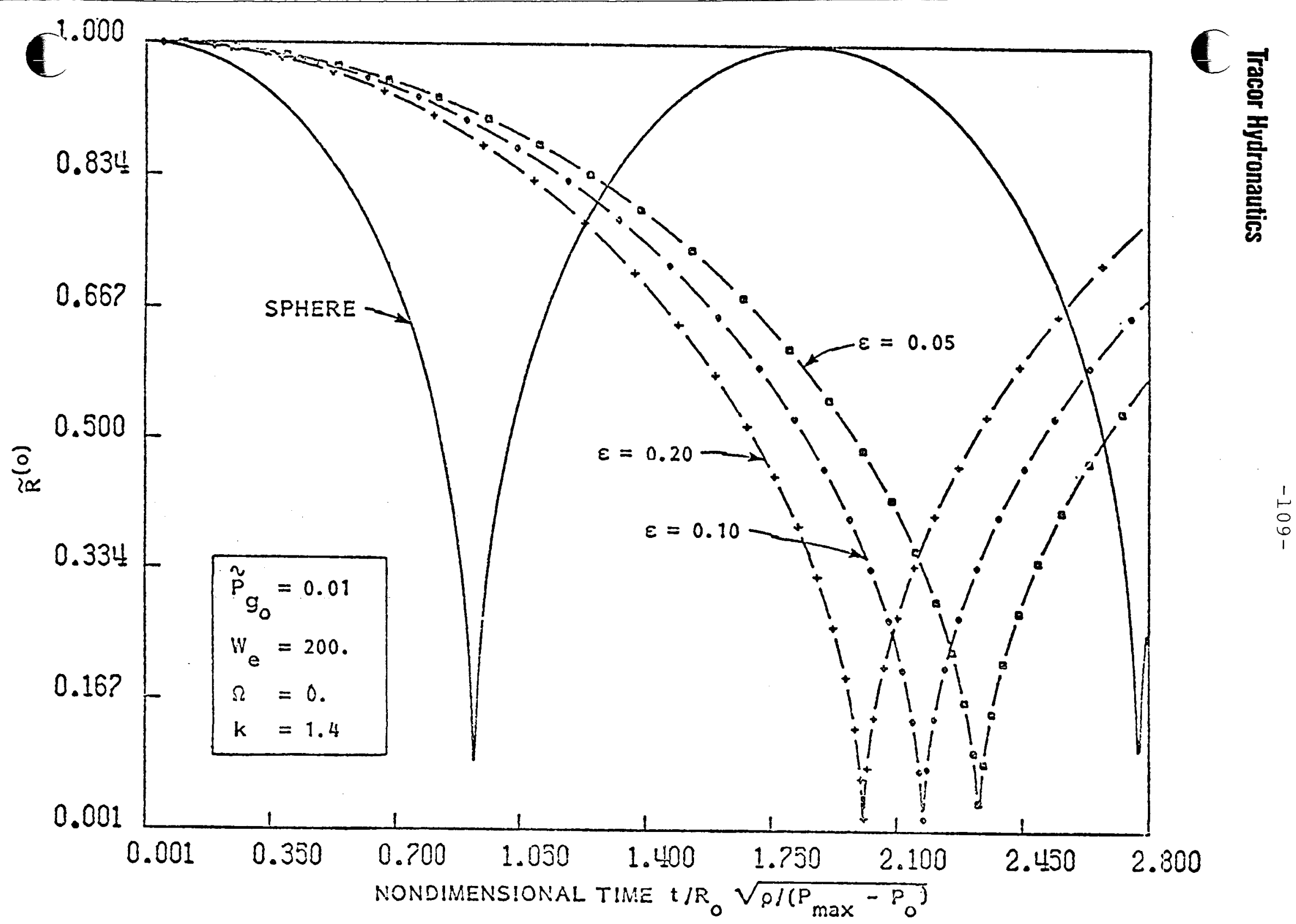

FIGURE 18 - COMPARISON OF COLLAPSE TIMES FOR SPHERICAL AND TOROIDAL BUBBLES 


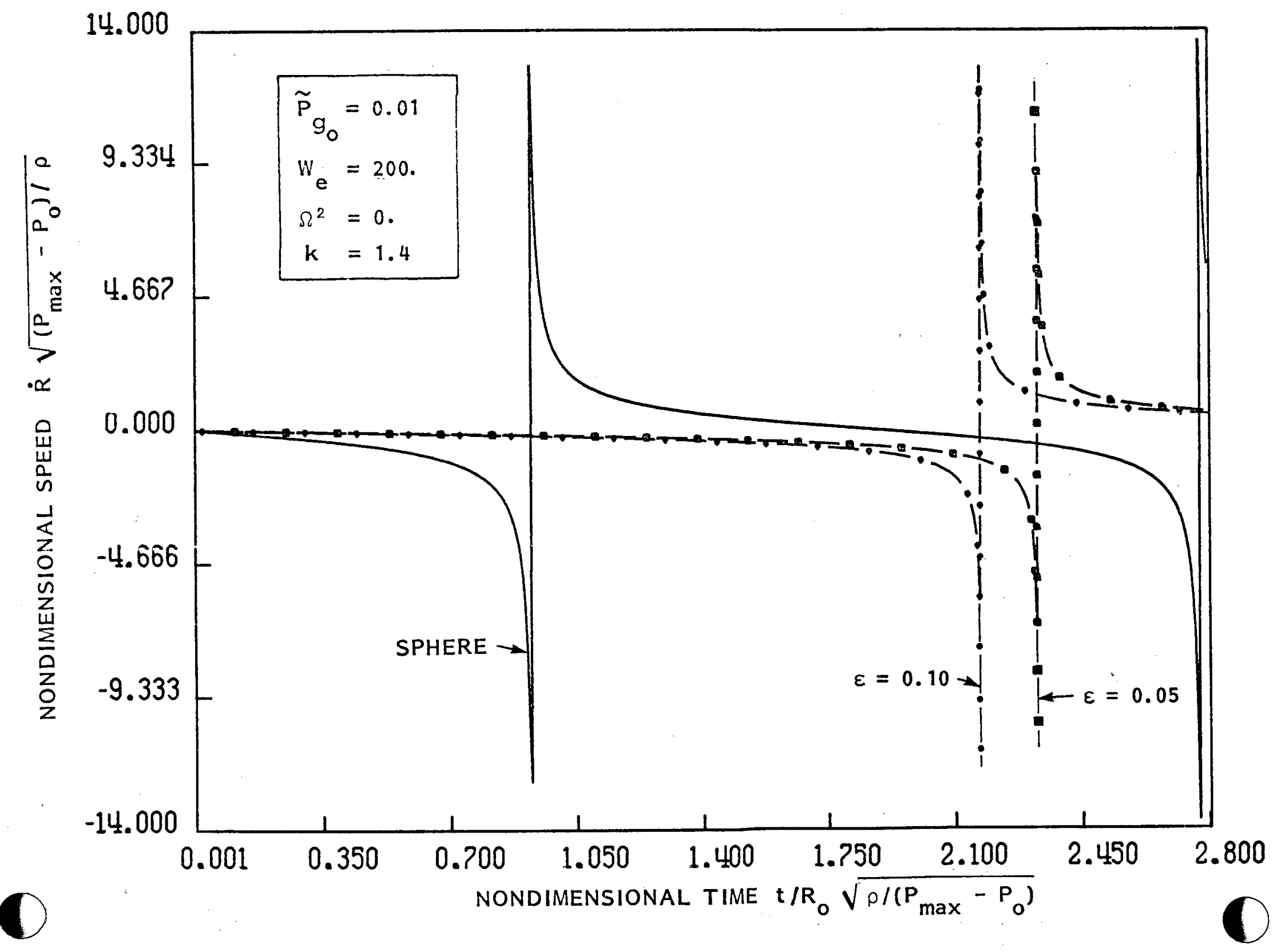

몽 


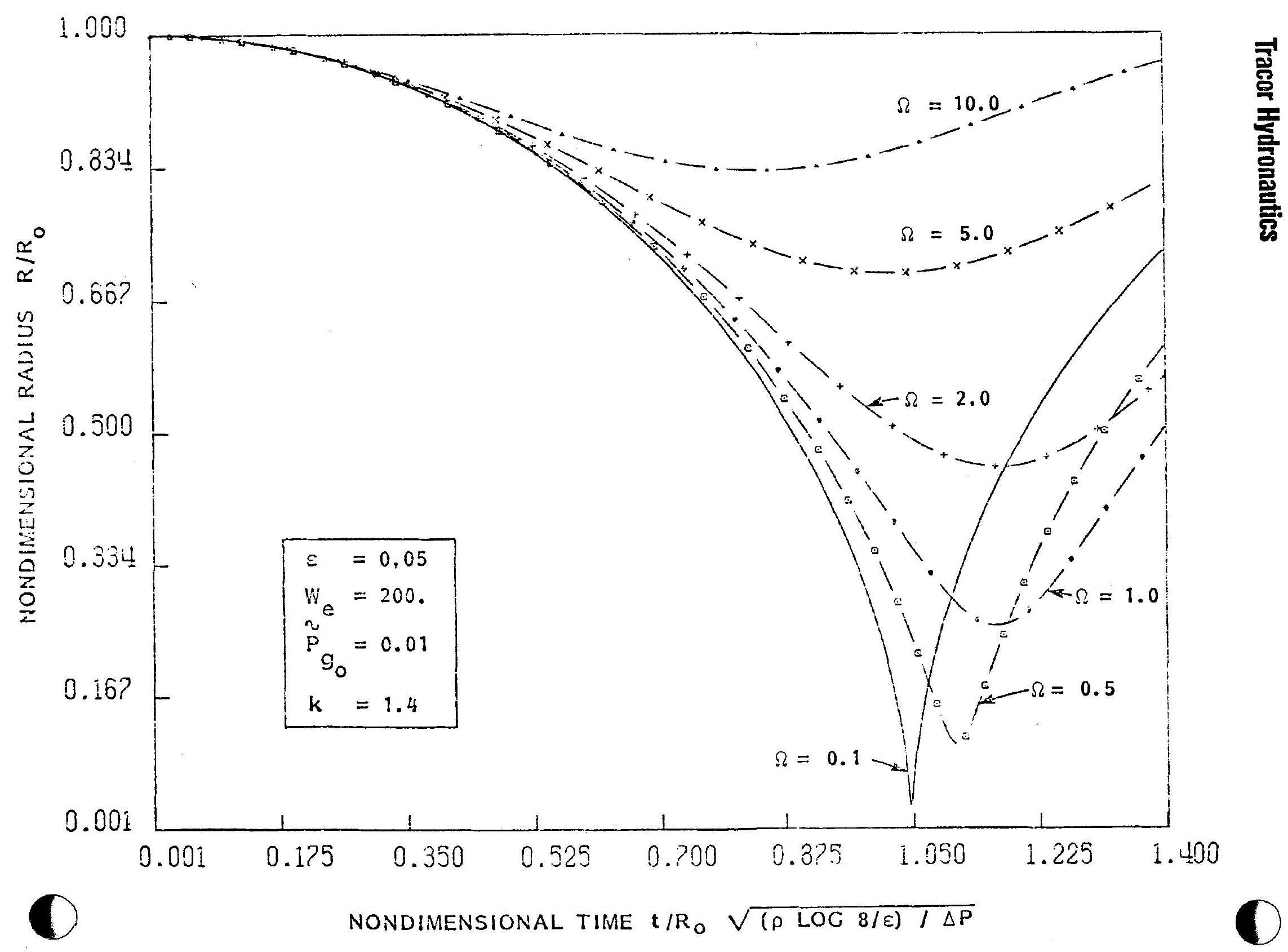




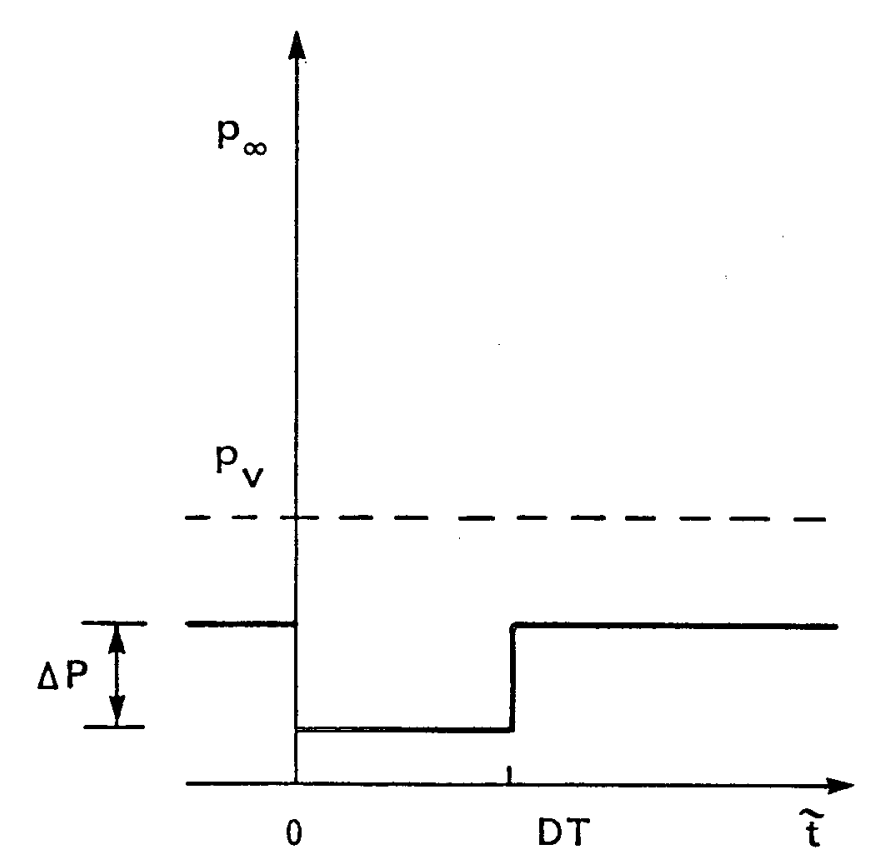

a. case $\mathscr{P}_{<} 0$

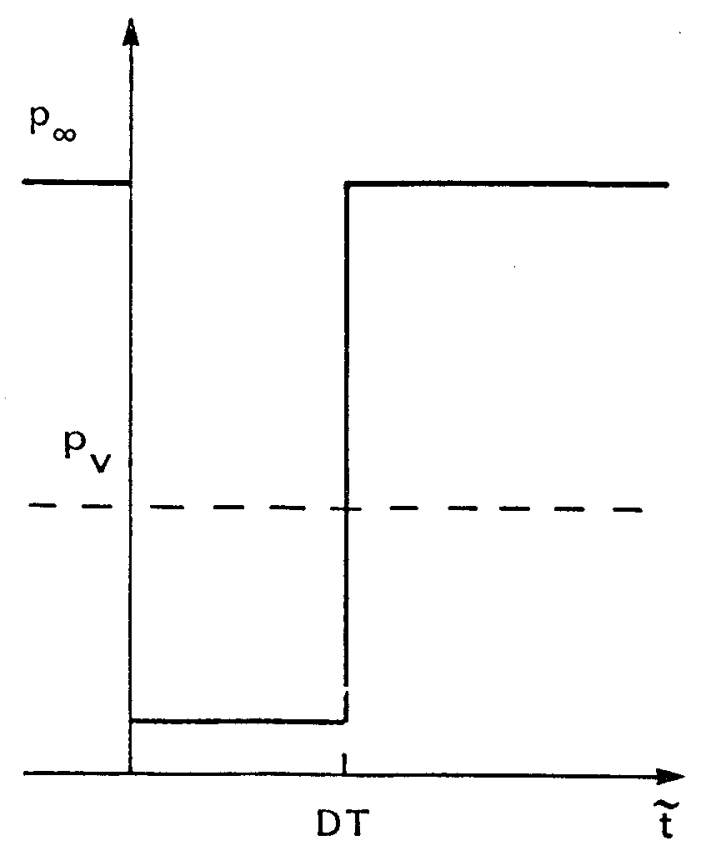

b. case $0<\mathscr{P}<1$

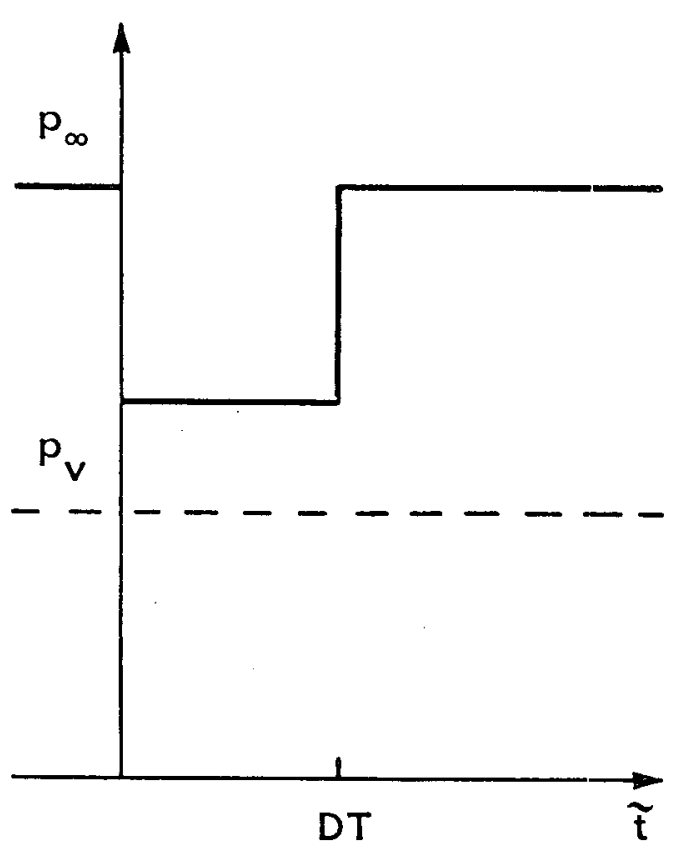

c. case $\mathscr{P}>1$

FIGURE 22 - VARIOUS CASES OF AMBIENT PRESSURE FUNCTIONS $\mathscr{P}=\left(P_{\infty}(0)-P V\right) / \triangle P$ 


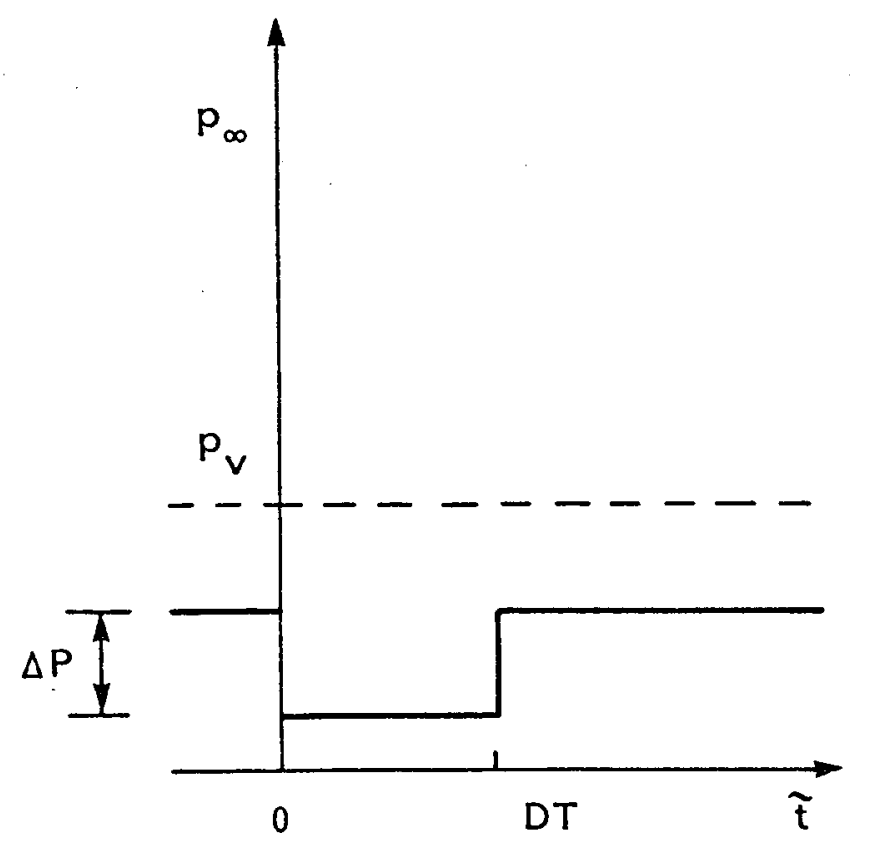

a. case $\mathscr{P}_{<} 0$

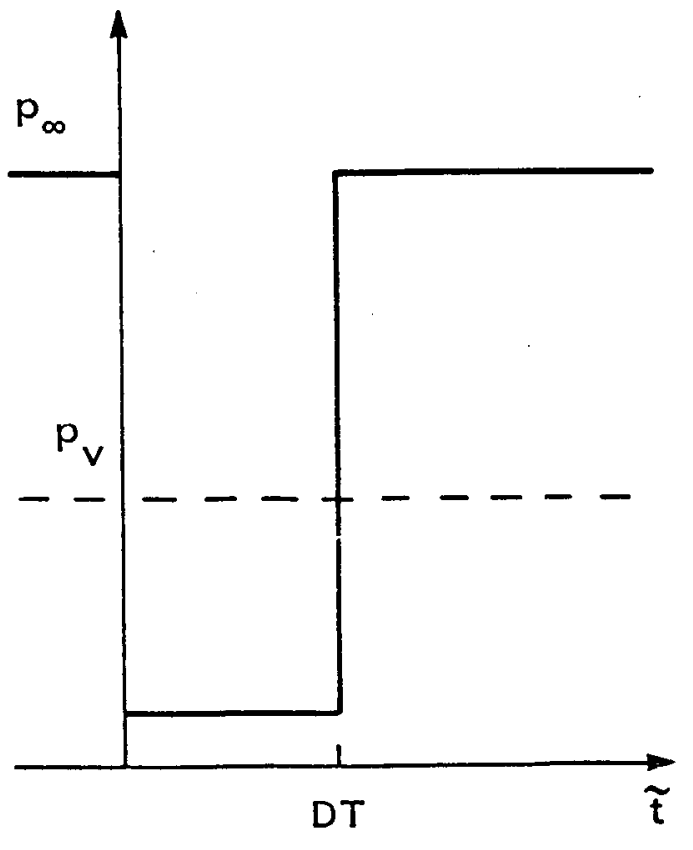

b. case $0<\mathscr{P}<1$

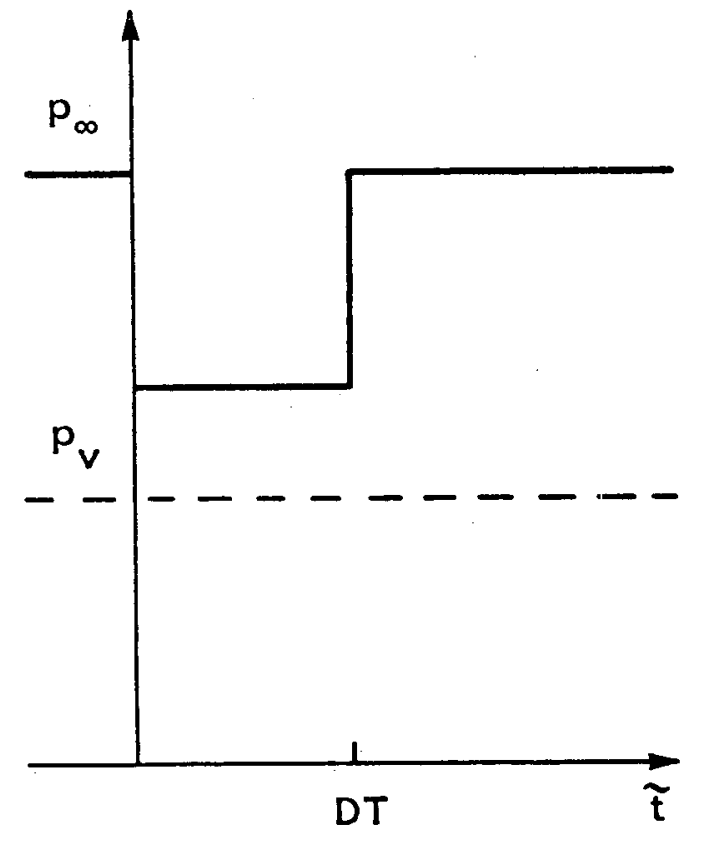

c. case $\mathscr{P}>1$ 
C

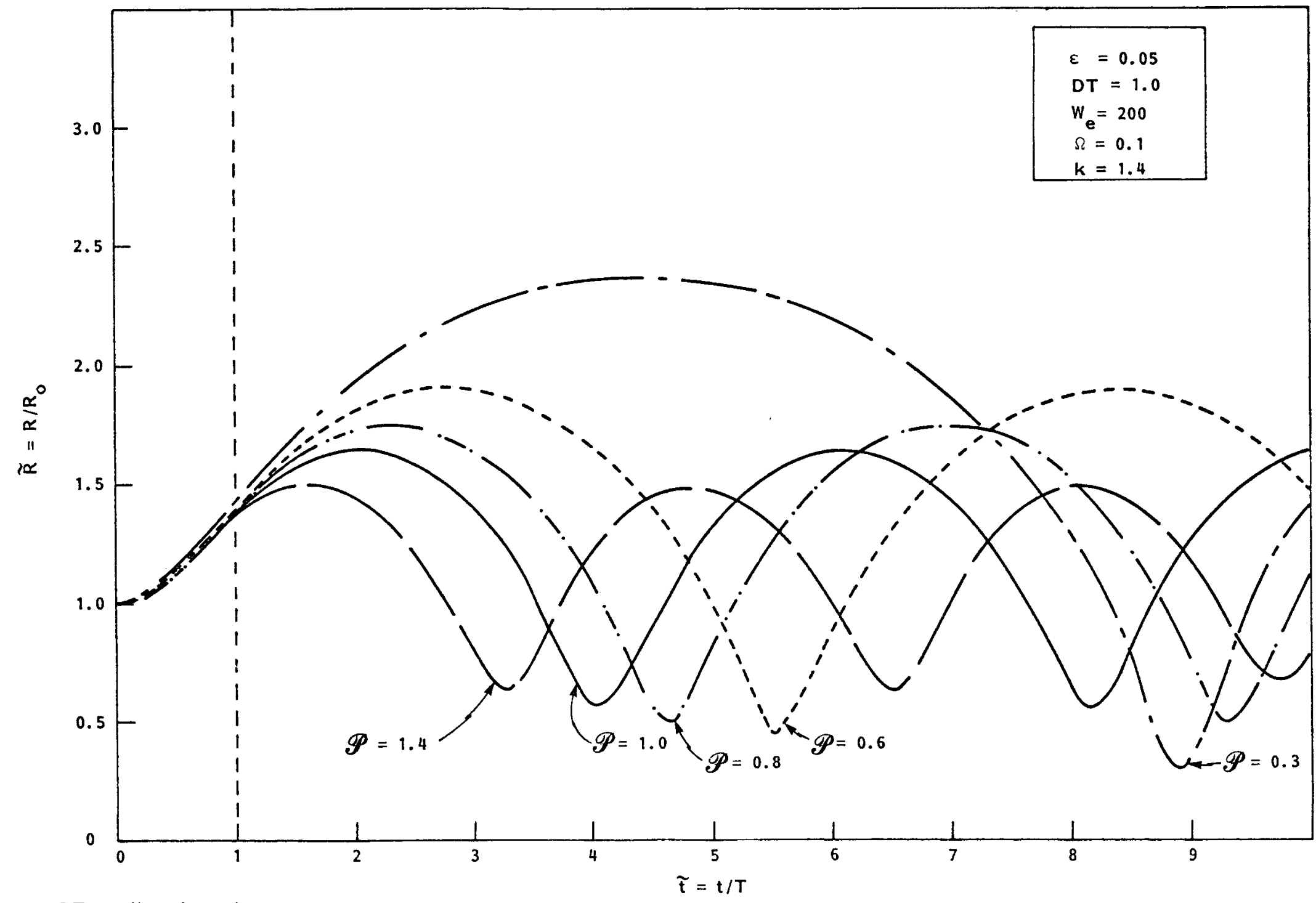

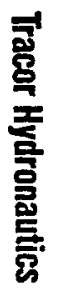

smaller than ring charac. oscill. time

FIGURE 23 - INFLUENCE OF THE PRESSURE DROP AMOUNT ON THE BUBBLE VORTEX RING BEHAVIOR $\mathscr{P}=\left(P_{\infty}(0)-P V\right) / \triangle P, D T=1$ 


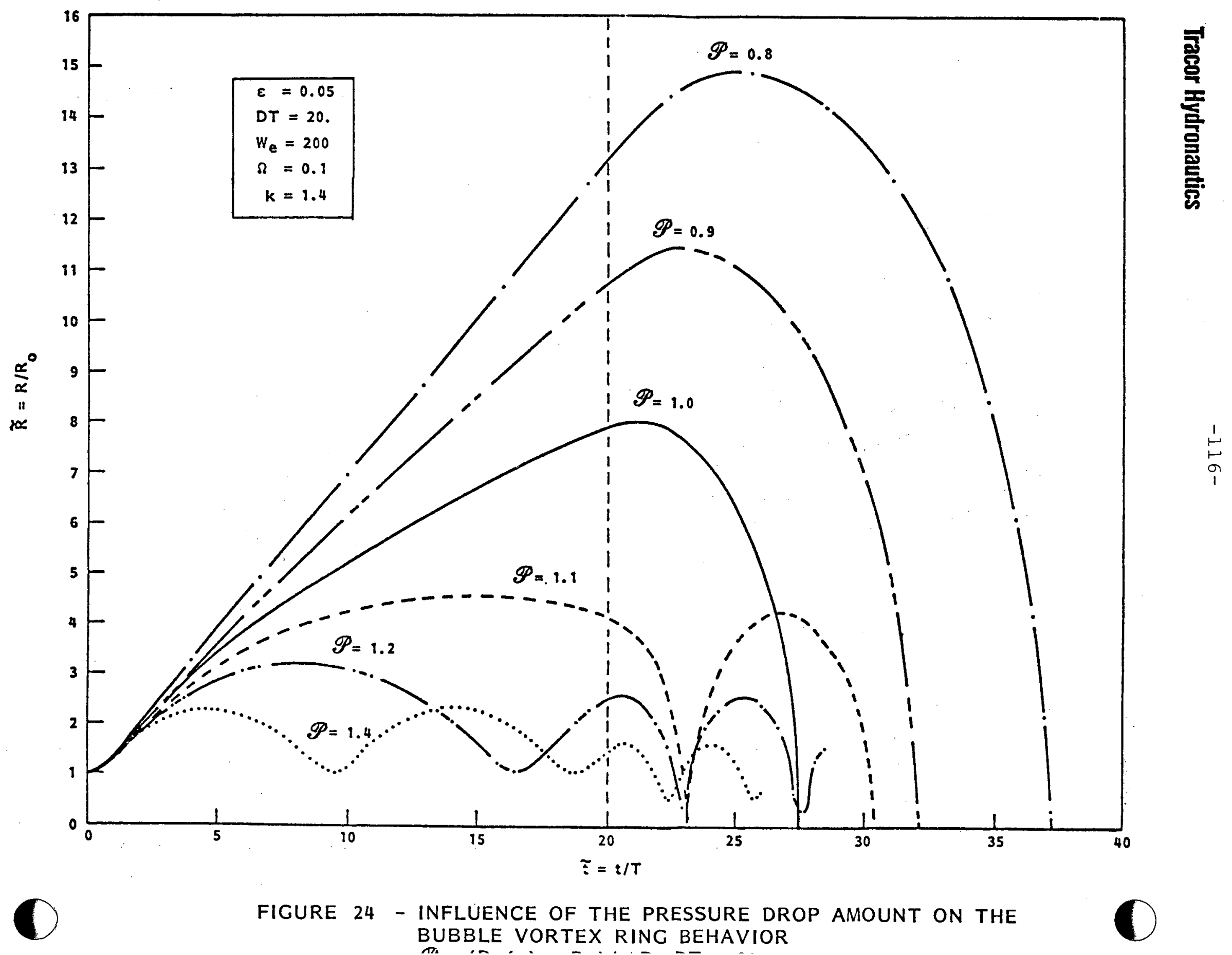




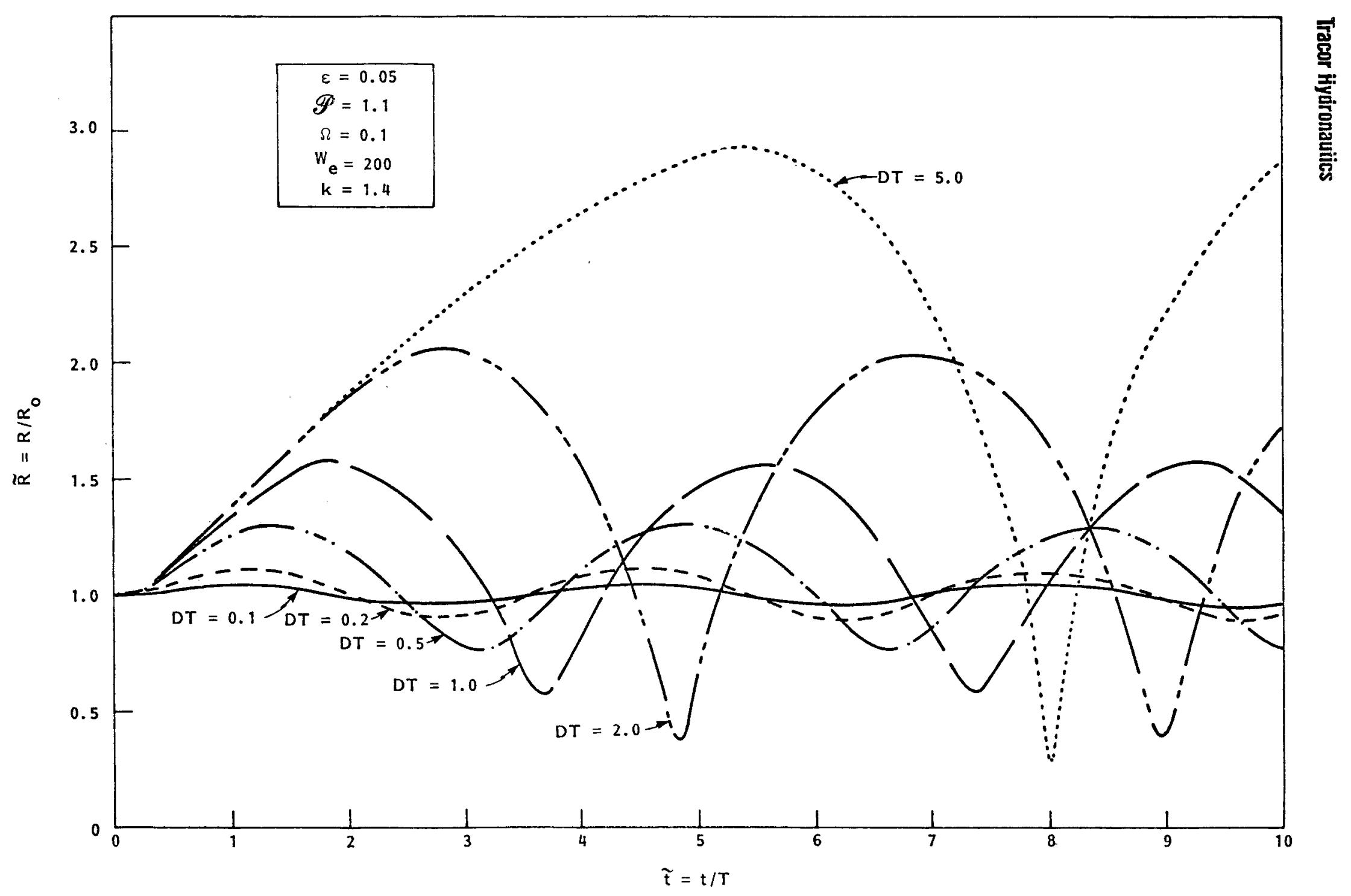

FIGURE 25 - INFLUENCE OF DURATION OF PRESSURE DROP ON BUBBLE VORTEX RINC BEHAVIOR 


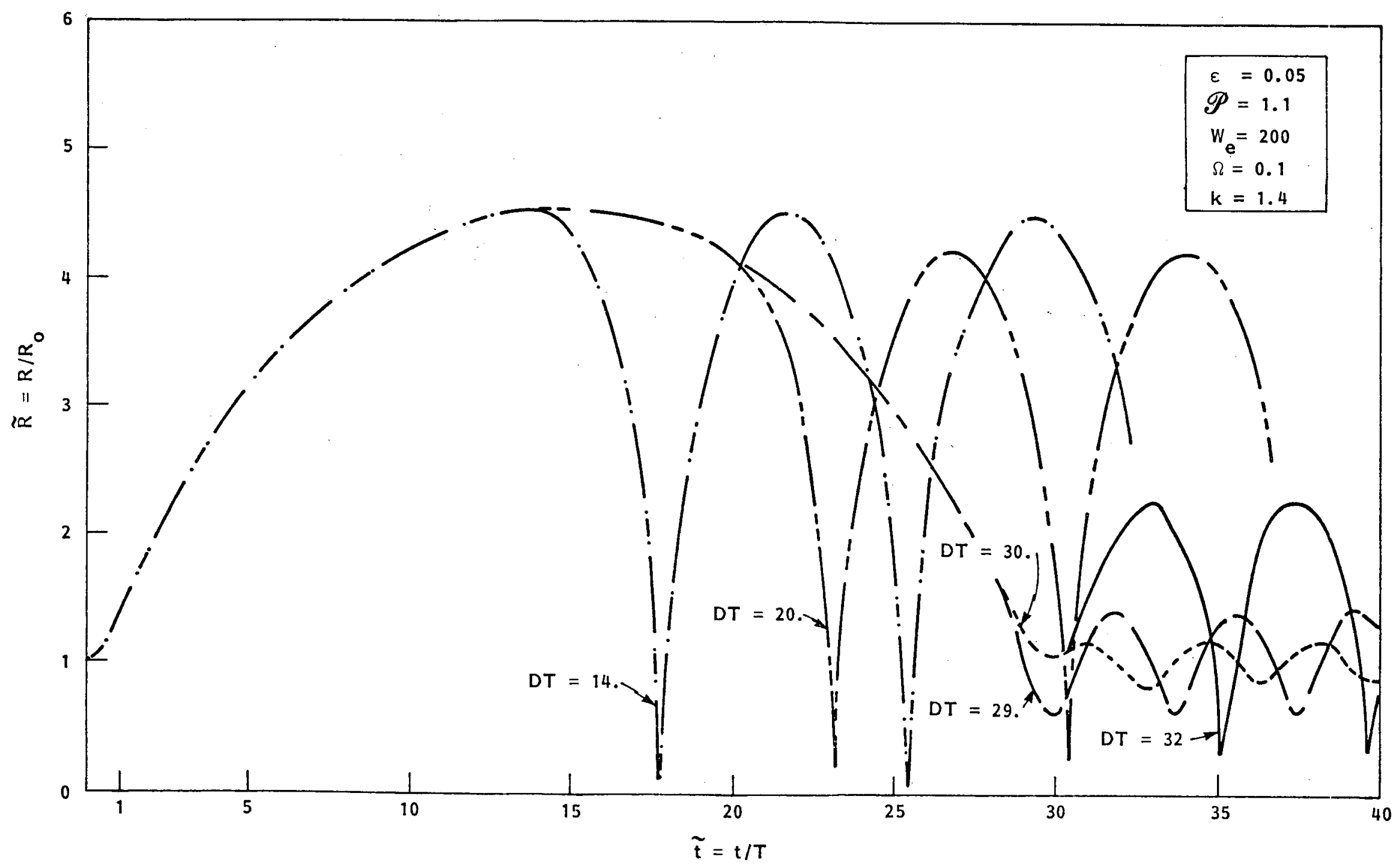

FIGURE 26 - INFLUENCE OF DURATION OF PRESSURE DROP ON BUBBLE VORTEX RINC BEHAVIOR 


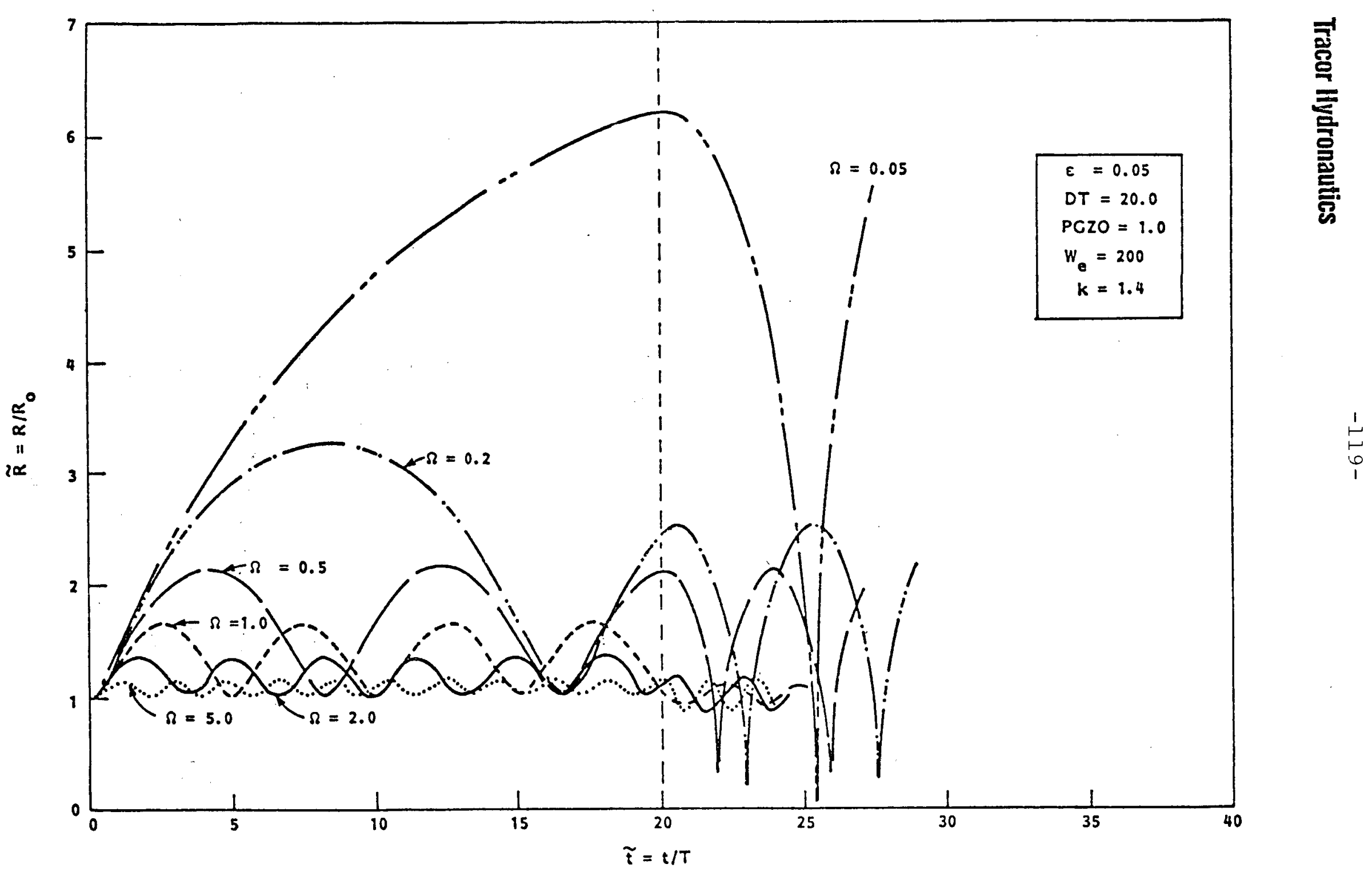

FIGURE 27 - INFLUENCE OF CIRCULATION ON BUBBLE VORTEX RINC BEHAVIOR 


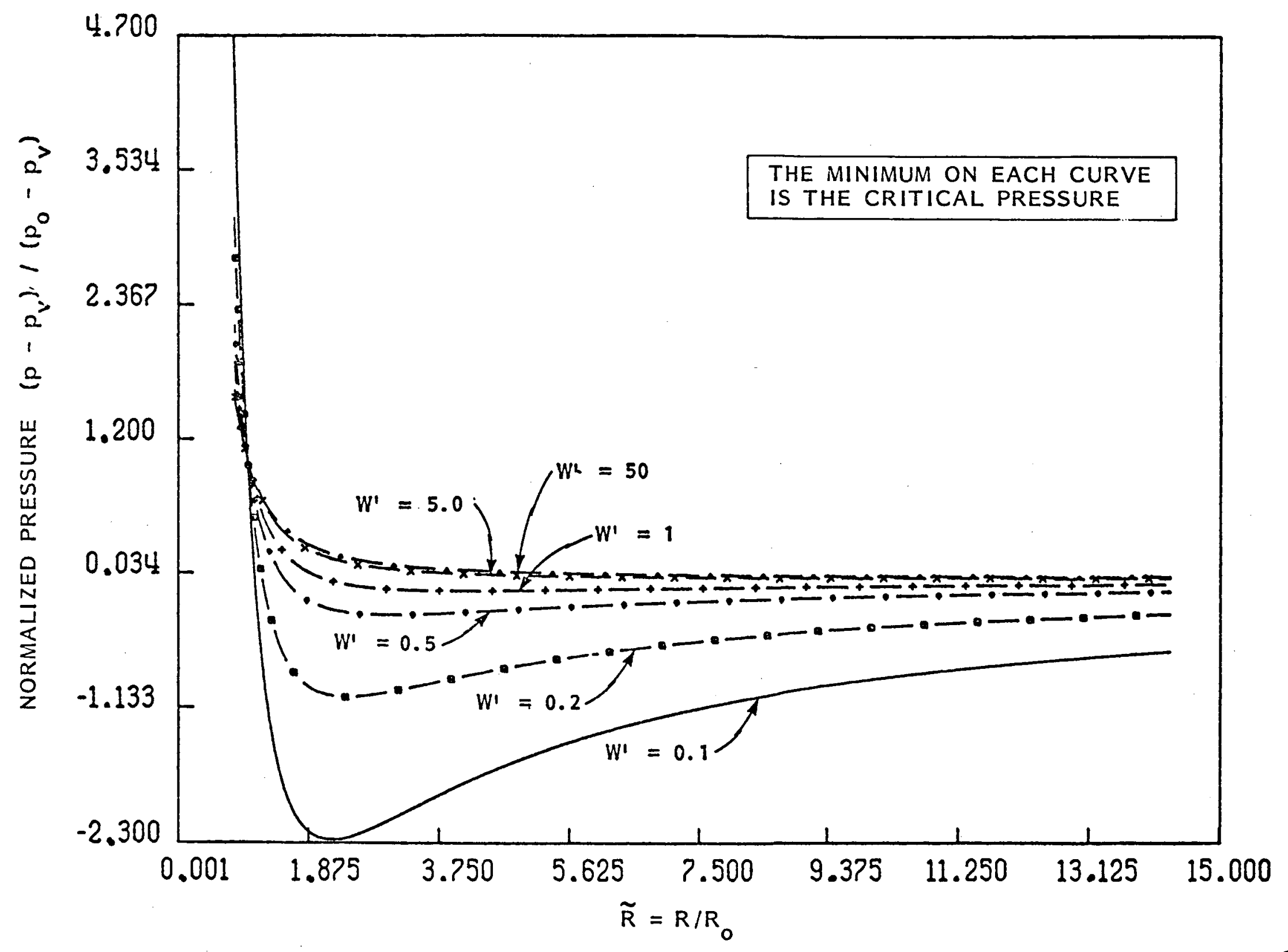

좀 
c

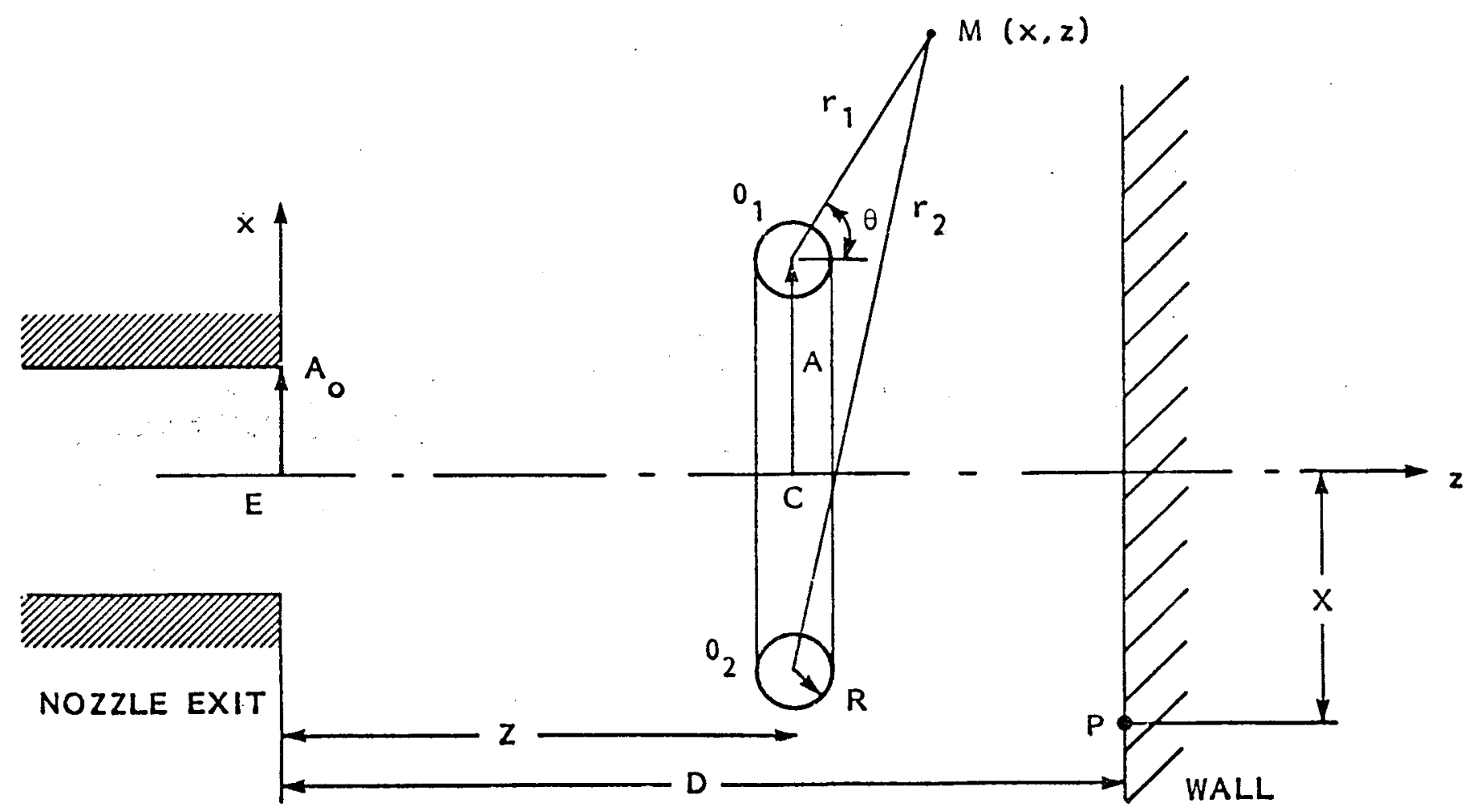

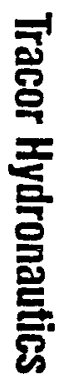

FIGURE 29 - DEFINITION DIAGRAM OF THE GEOMETRY 
NONDIMENSIONAL AXIAL DISTANCE FROM NOZZLE, $\bar{z}=2 / \mathrm{A}_{0}$

FIGURE 30 - TRAJECTORIES OF RING VORTICES EMITTED AT CONSTANT

FREQUENCY, $\varepsilon=0.1, W_{\mathrm{e}}=200, \Omega=20, \overline{\mathrm{f}}=1$ 


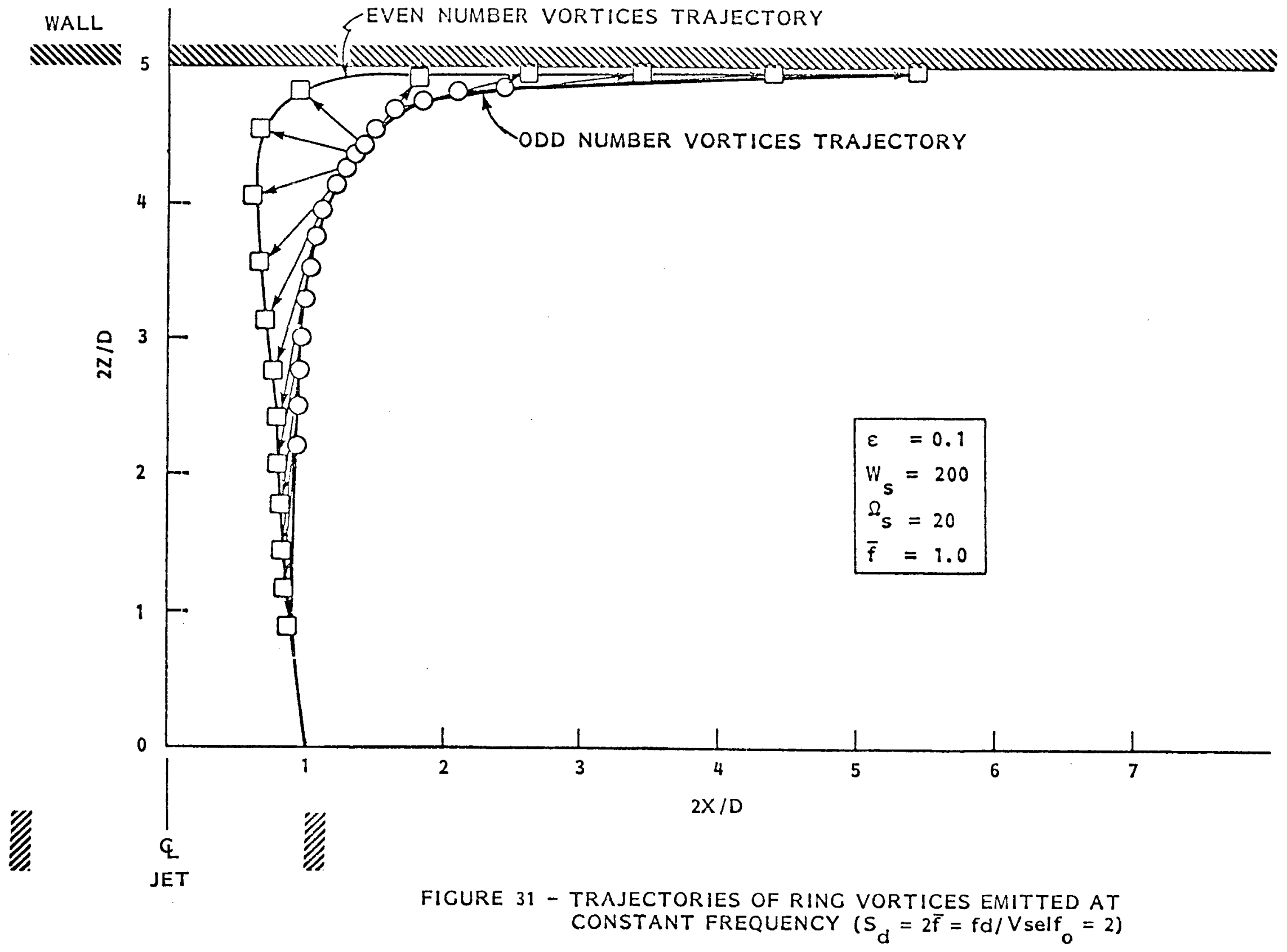




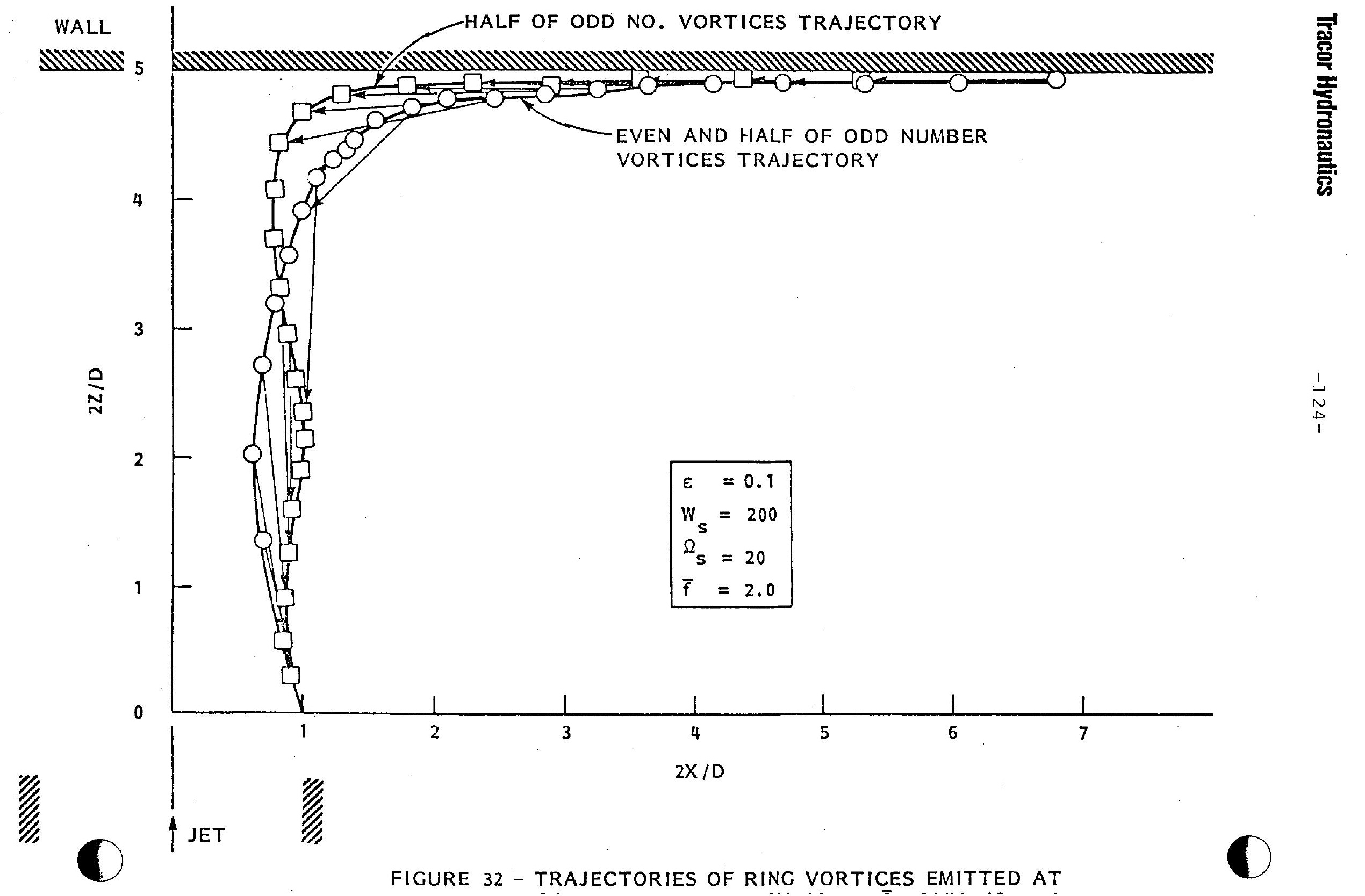




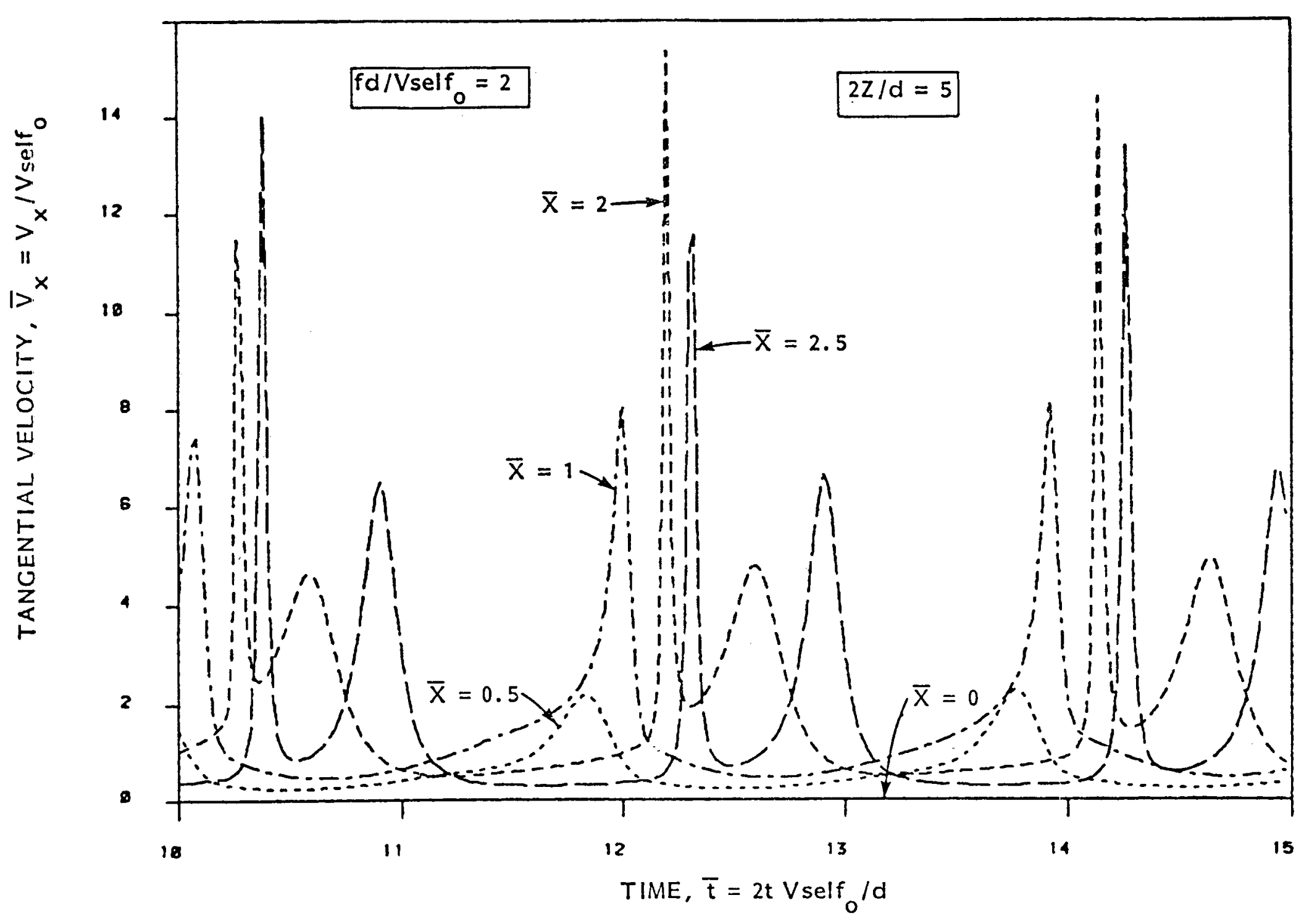

검
홈
돟
홀
핼

FIGURE 33 - TANGENTIAL VELOCITIES INDUCED AT DIFFERENT LOCATIONS, $X$, ON THE IMPACTED WALL VERSUS TIME 


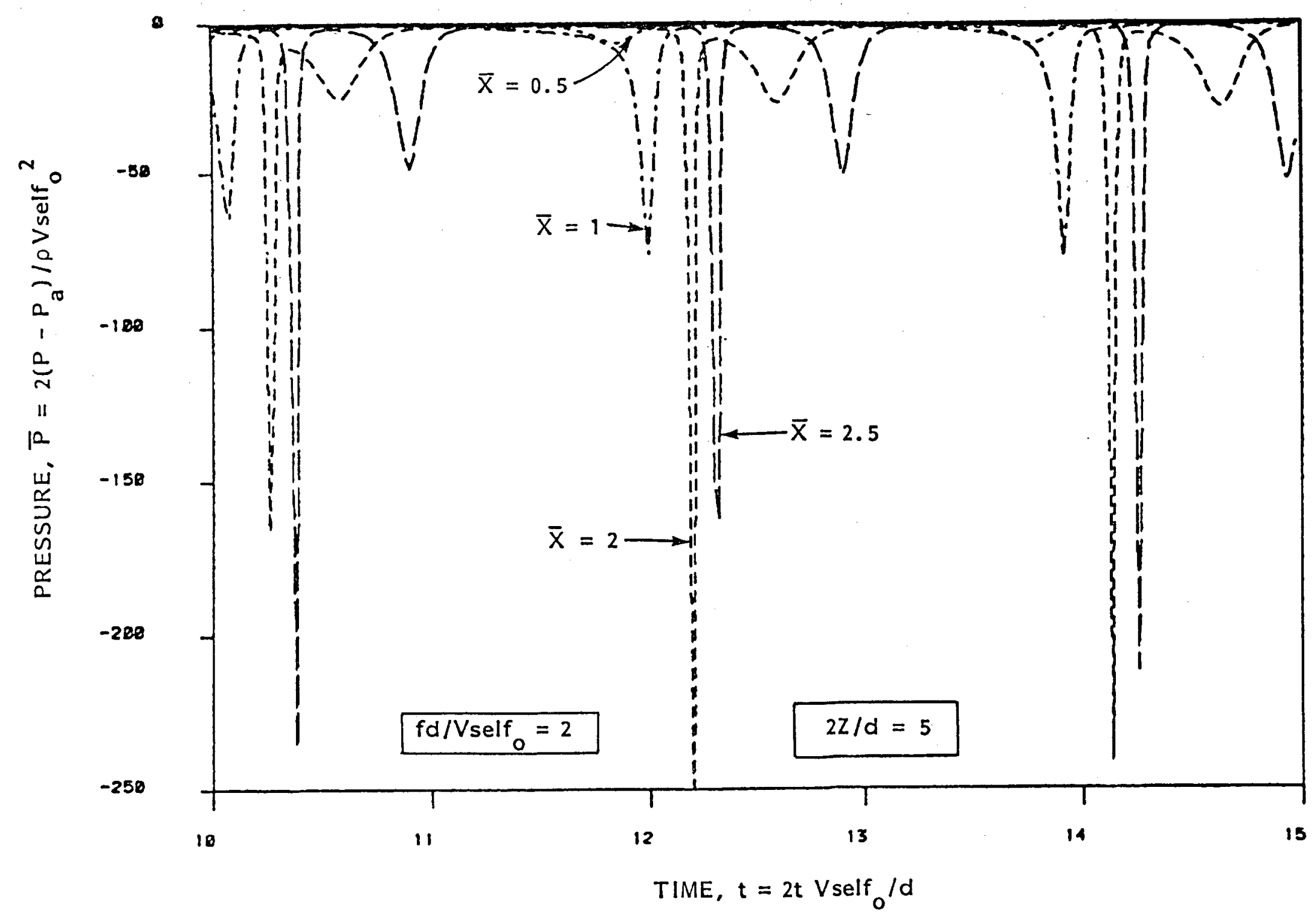

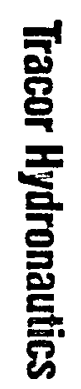

FIGURE 34 - PRESSURES INDUCED AT DIFFERENT LOCATIONS, $\bar{x}$, ON THE IMPACTED W!ALL, VERSUS TIME 


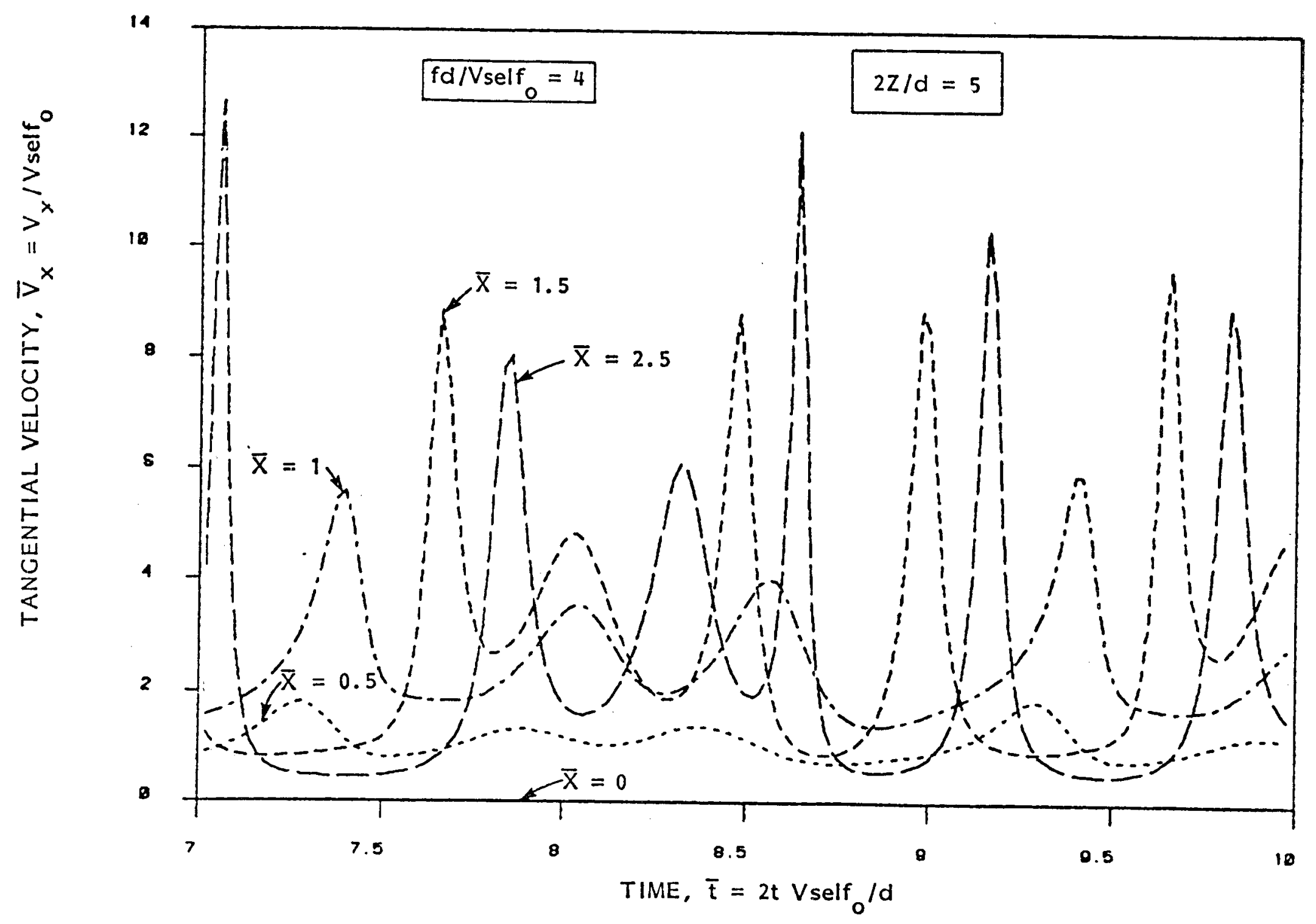

高

FICURE 35 - TANGENTIAL VELOCITIES INDUCED AT DIFFERENT LOCATIONS, $\bar{x}$, ON THE IMPACTED WALL VERSUS TIME 


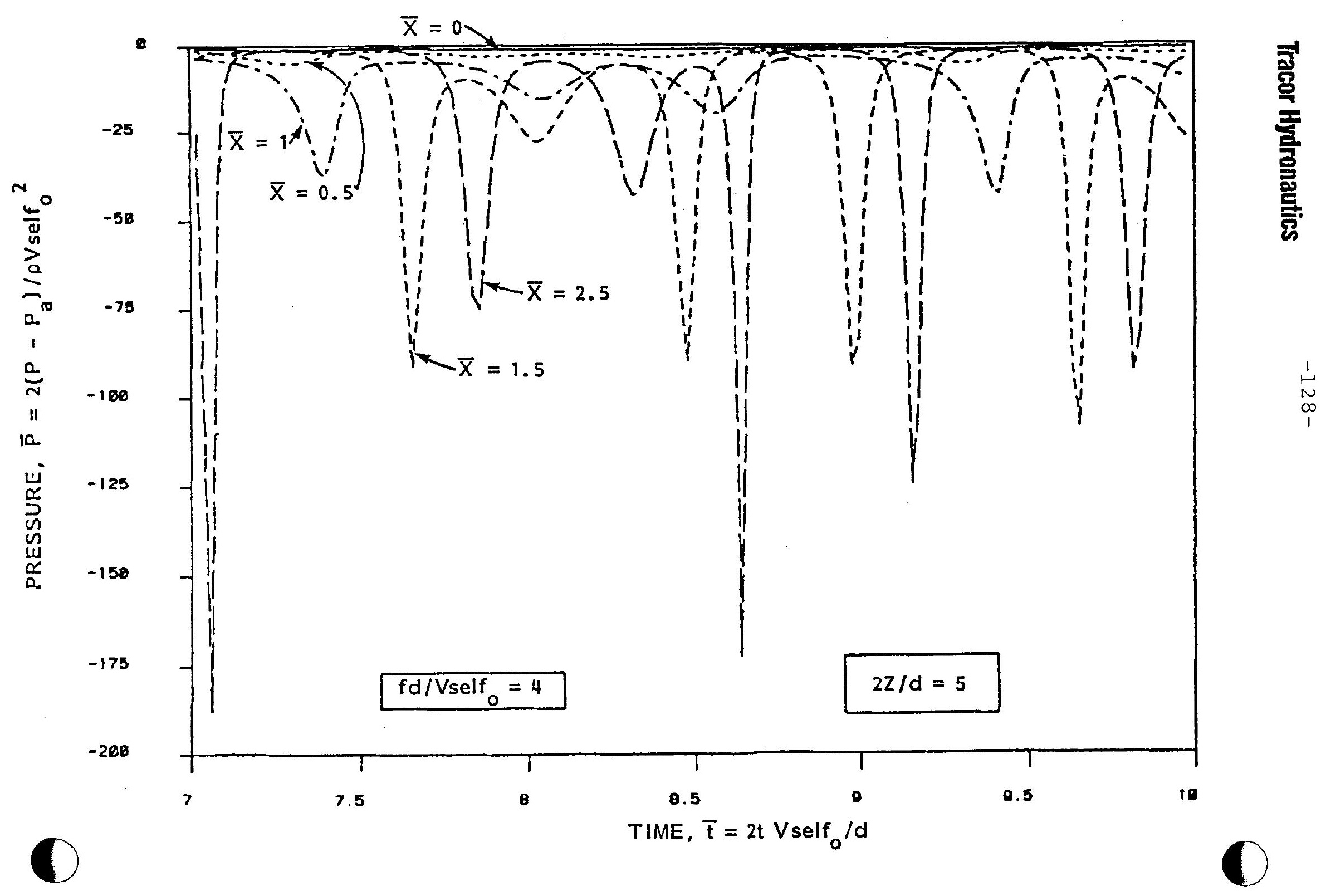




\section{Tracor Hydronautics}

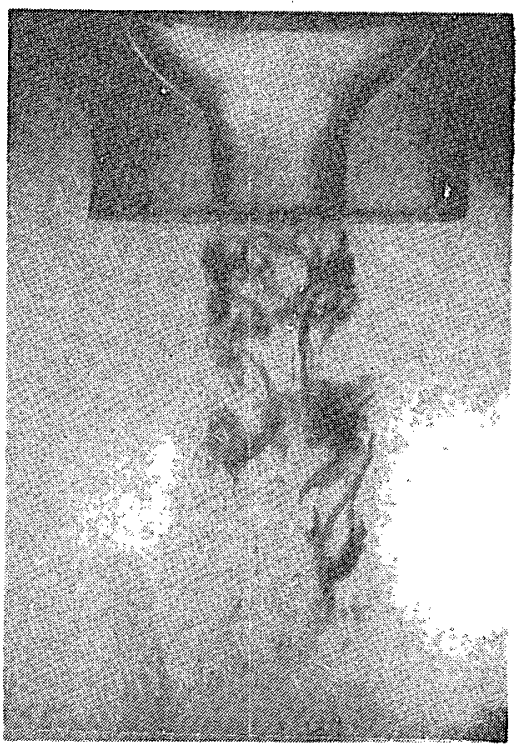

SMITH NOZZLE

$$
\begin{aligned}
& \mathrm{Pa}=28 \mathrm{psi} \\
& \Delta \mathrm{P}=90 \mathrm{psi} \\
& \sigma \approx 0.31
\end{aligned}
$$

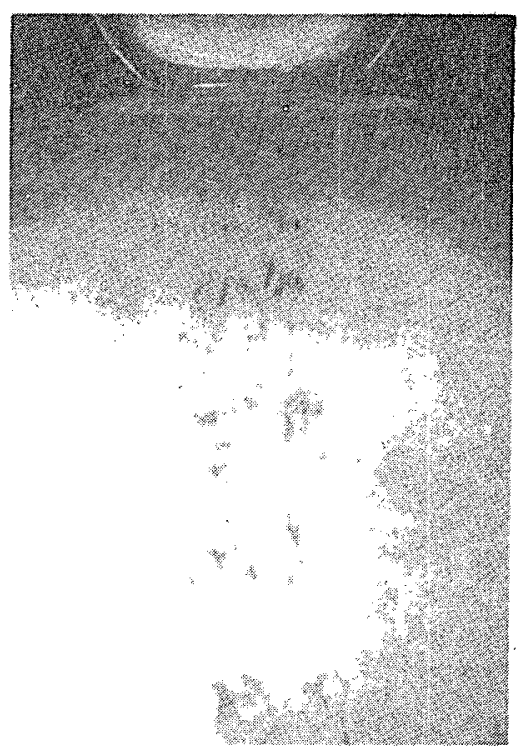

CAVIJET NOZZLE NO LIP

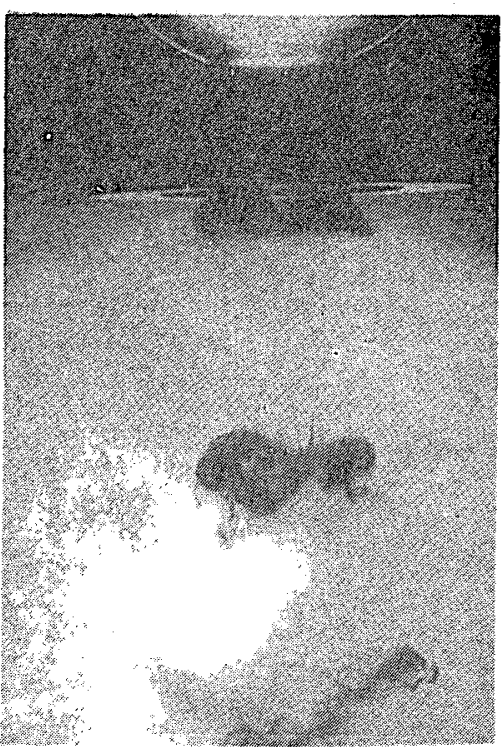

CAVIJET NOZZLE THICK LIP

FIGURE 37 - COMPARISON OF CAVITATION APPEARANCE IN THREE NOZZLE TYPES (ONLY THE LAST IS SELF-RESONATING) 


\section{Tracor Hydronautics}

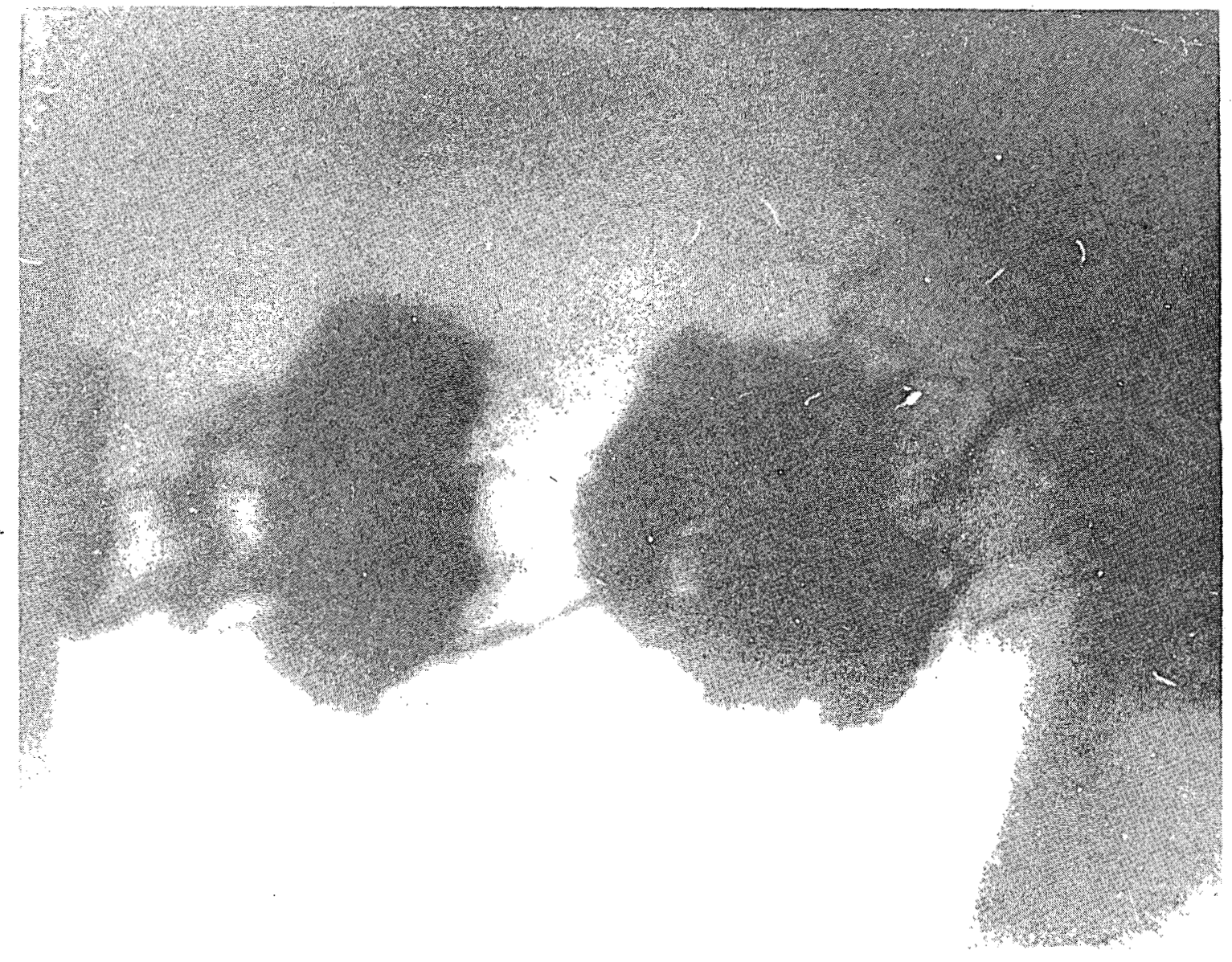

FIGURE 38 - APPEARANCE OF CAVITATING STRATOJET AT A

CAVITATION NUMBER OF $0.25, M=0.118, f=9.5 \mathrm{KHz}$ 


\section{Tracor Hydronautics}

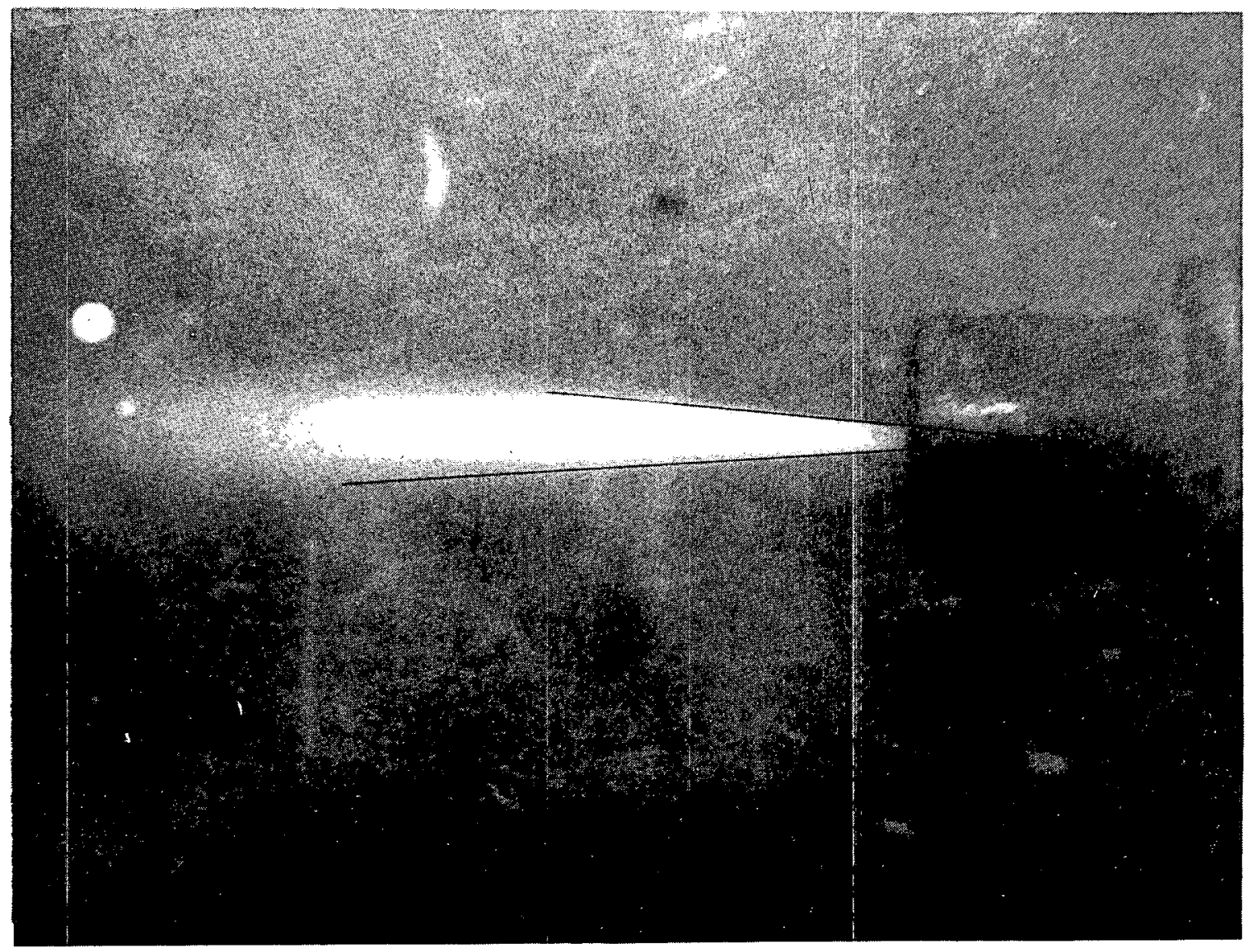

FIGURE 39 - APPEARANCE OF CAVITATING CAVIJET AT A VERY LOW CAVITATION NUMBER, $\sigma \simeq 0.023, \triangle P \sim 650 \mathrm{psi}$ 

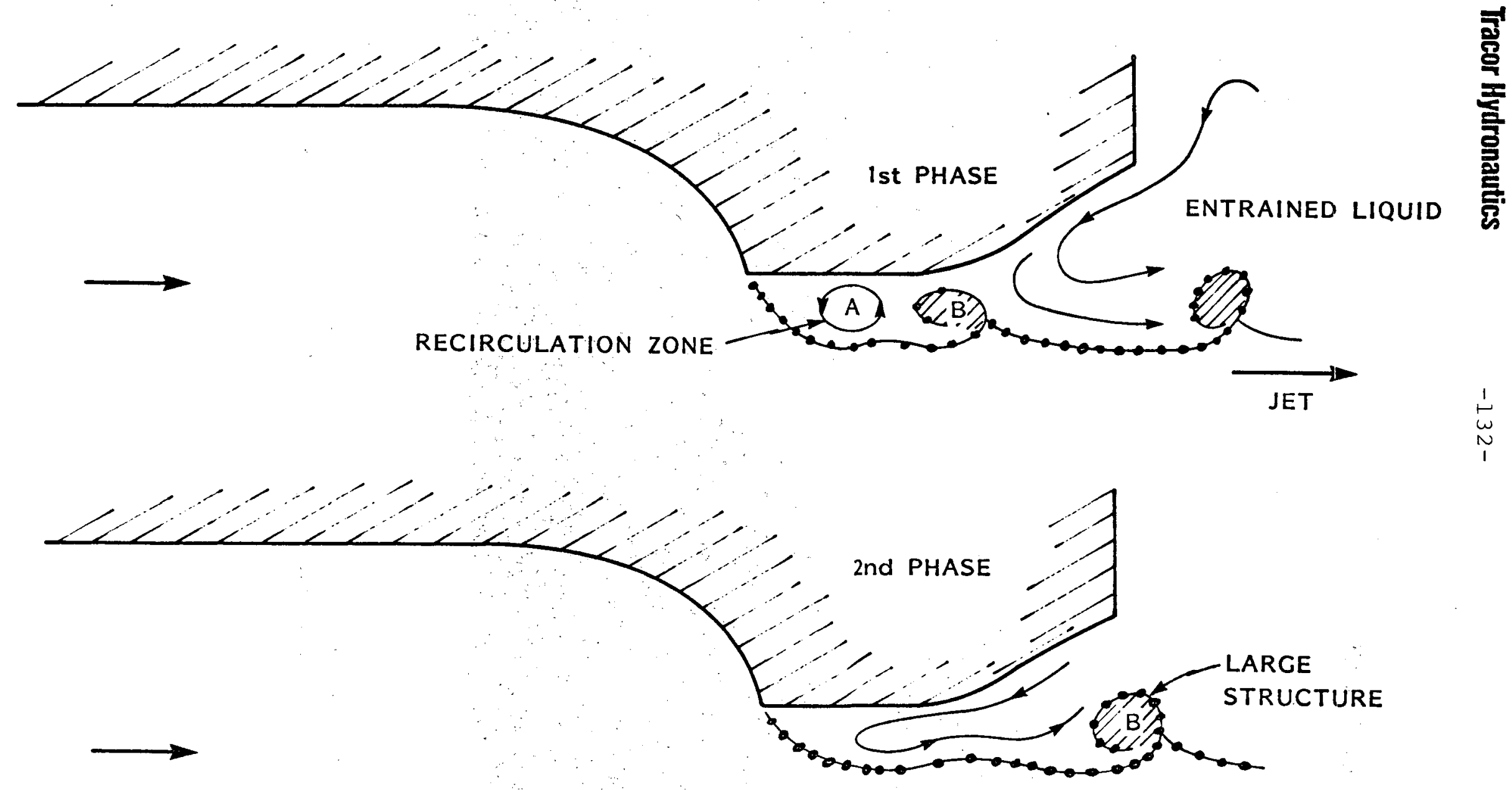

FIGURE 40 - SCHEMATIC OF NOZZLE LIP-JET INTERACTION AND FEED-BACK MECHANISM 
$\mathcal{E}$
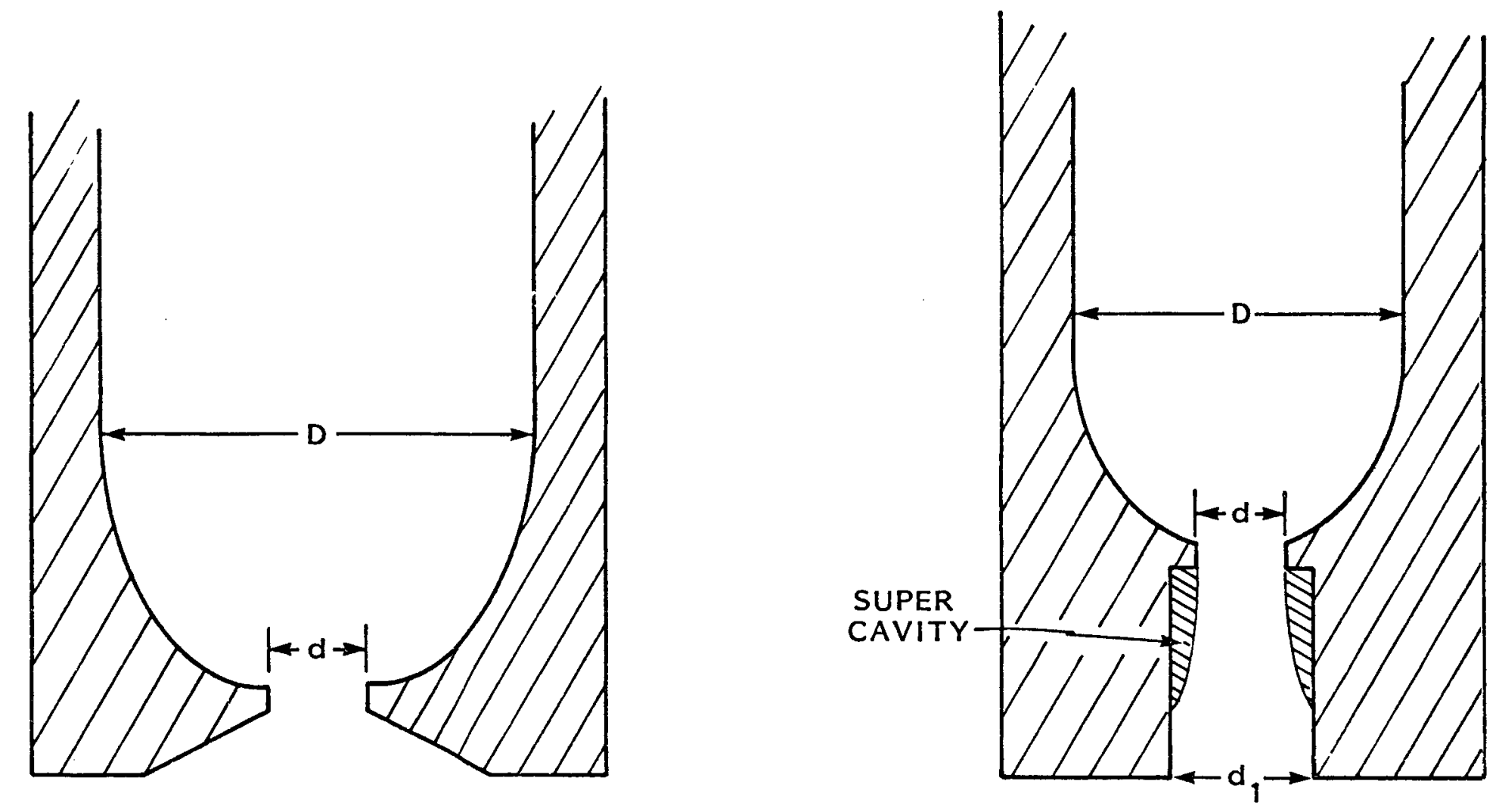

콩

$\underset{\omega}{\omega}$

a. SHARP EDGE STRATOJET

b. LONG ORIFICE STRATOJET

FIGURE 41 - LOW-SIGMA STRATOJET NOZZLES: a. AVOIDED SUPER CAVITY

b. CONTROLLED SUPER CAVITY 


\section{Tracor Hydronautics}

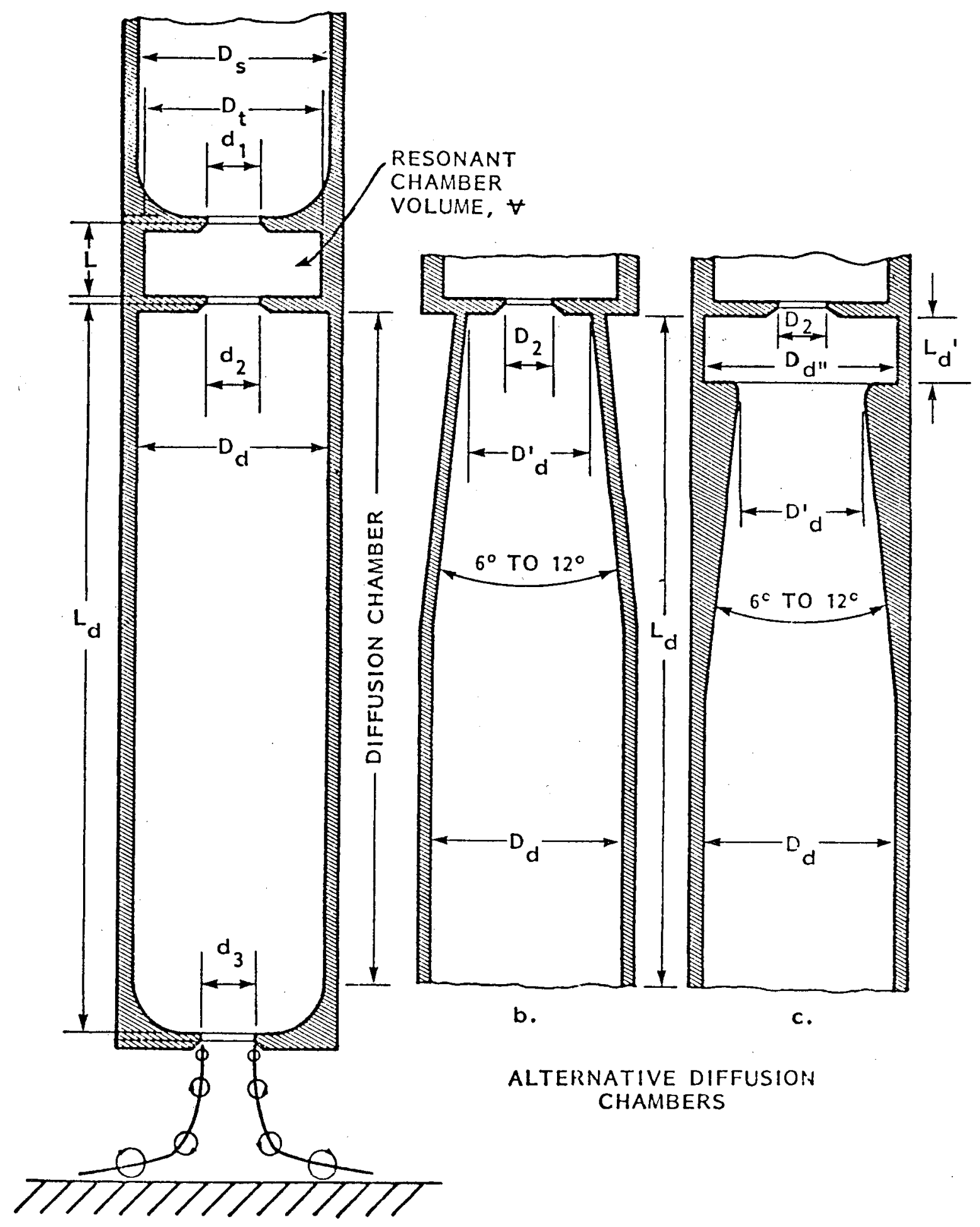

a. BASIC DESICN

FIGURE 42 - CONCEPTS FOR PULSER-FED CAVIJET ${ }^{\circledR}$ NOZZLES 
$\frac{1}{4}$

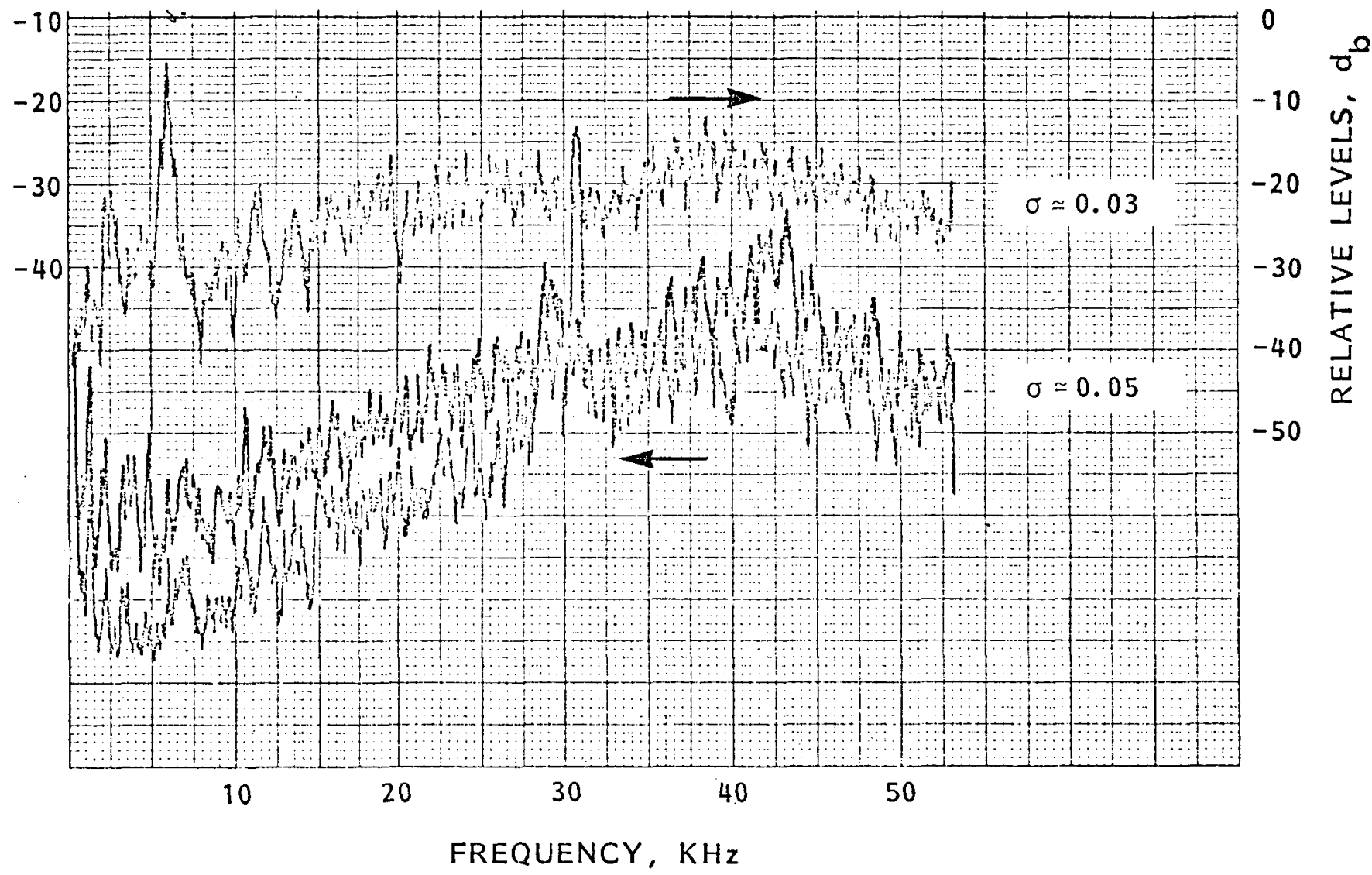


Tracor Hydronautics

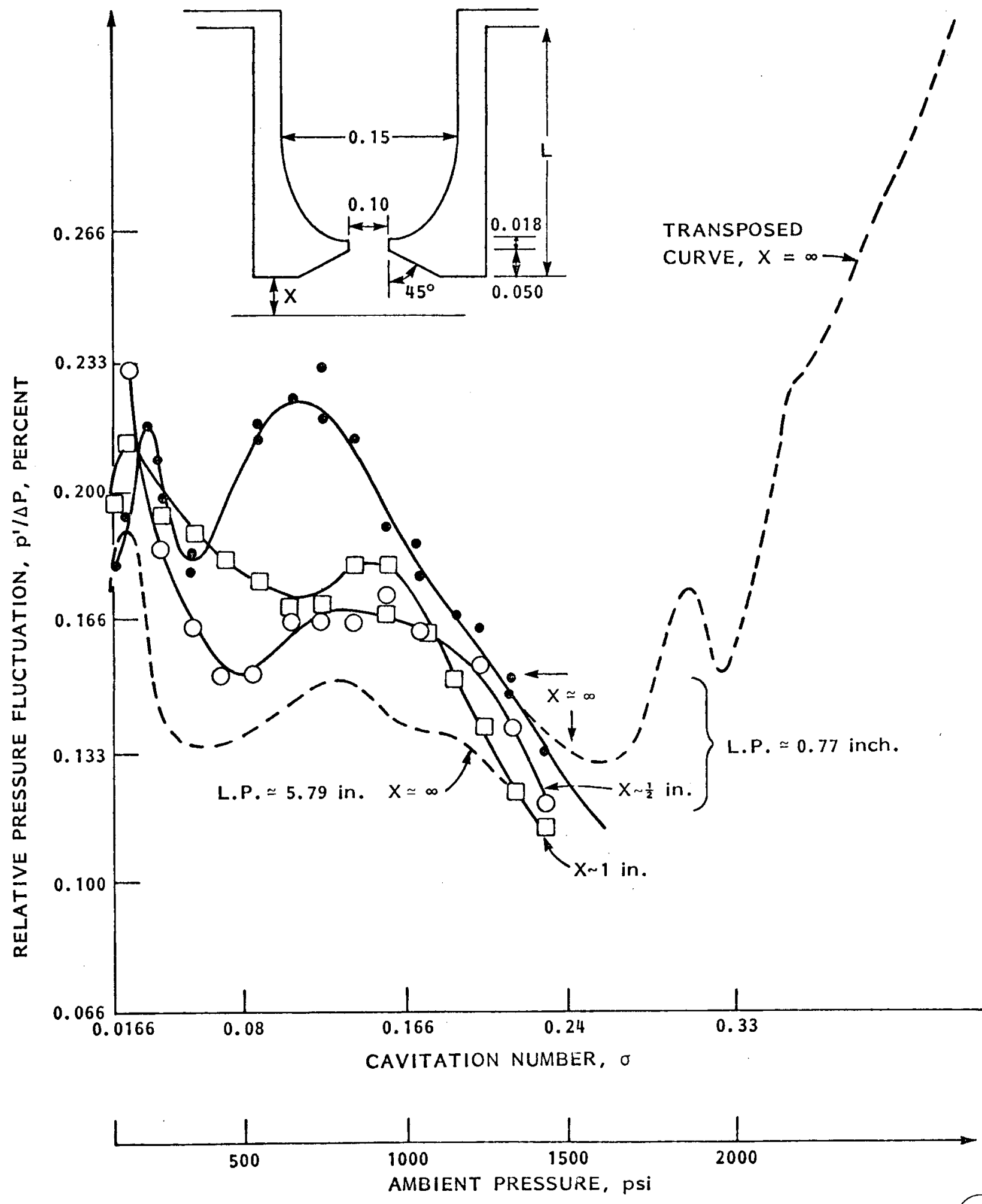

FIGURE 44 - INFLUENCE OF THE STANDOFF DISTANCE, $X$, AND OF THE ORCAN PIPE, L, ON THE PRESSURE FLUCTUATIONS GENERATED BY A SHARP EDGE NOZZLE AT LOW CAVITATION NUMBERS. $\triangle P=6000$ psi, 


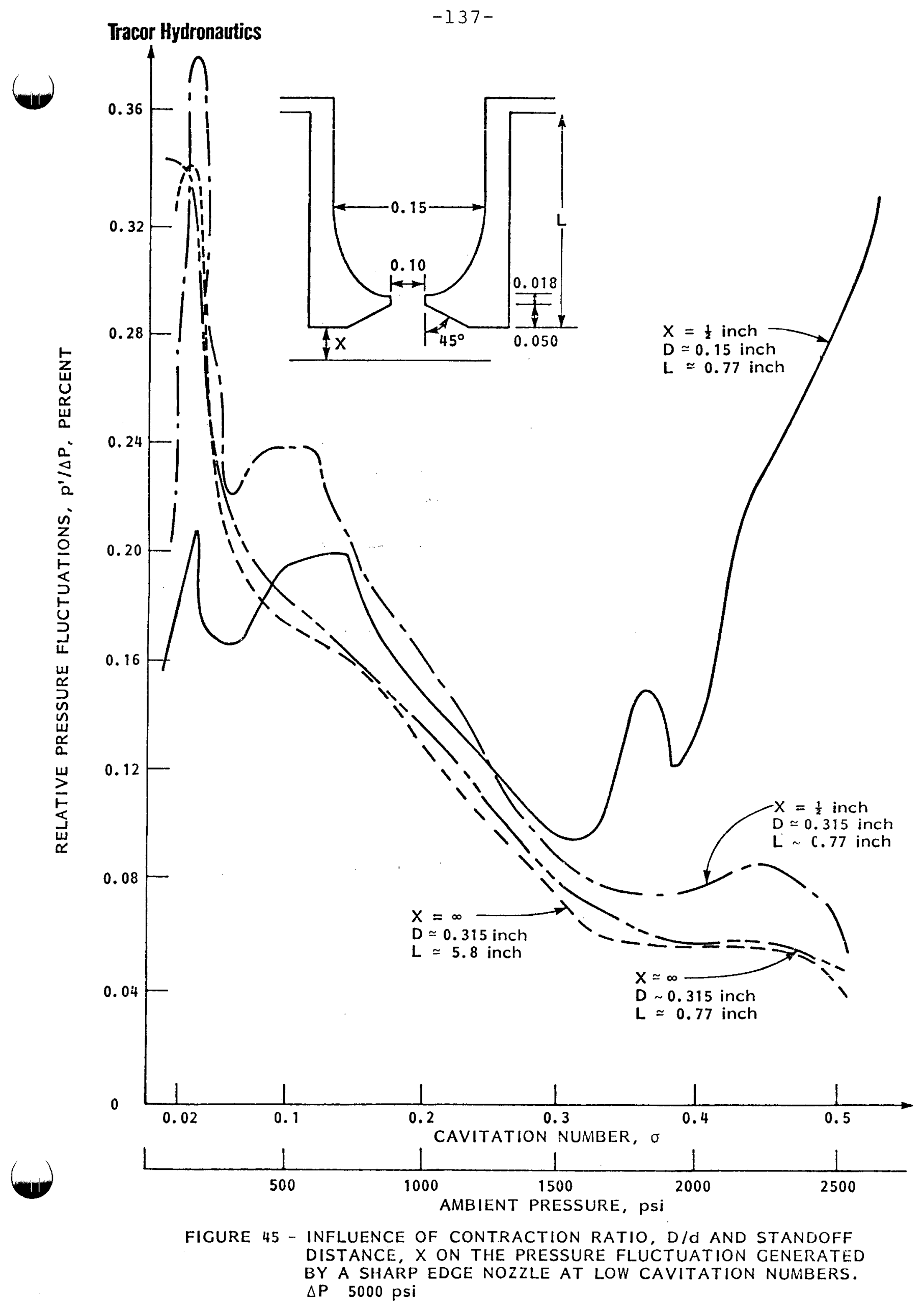


Tracor Hydronautics

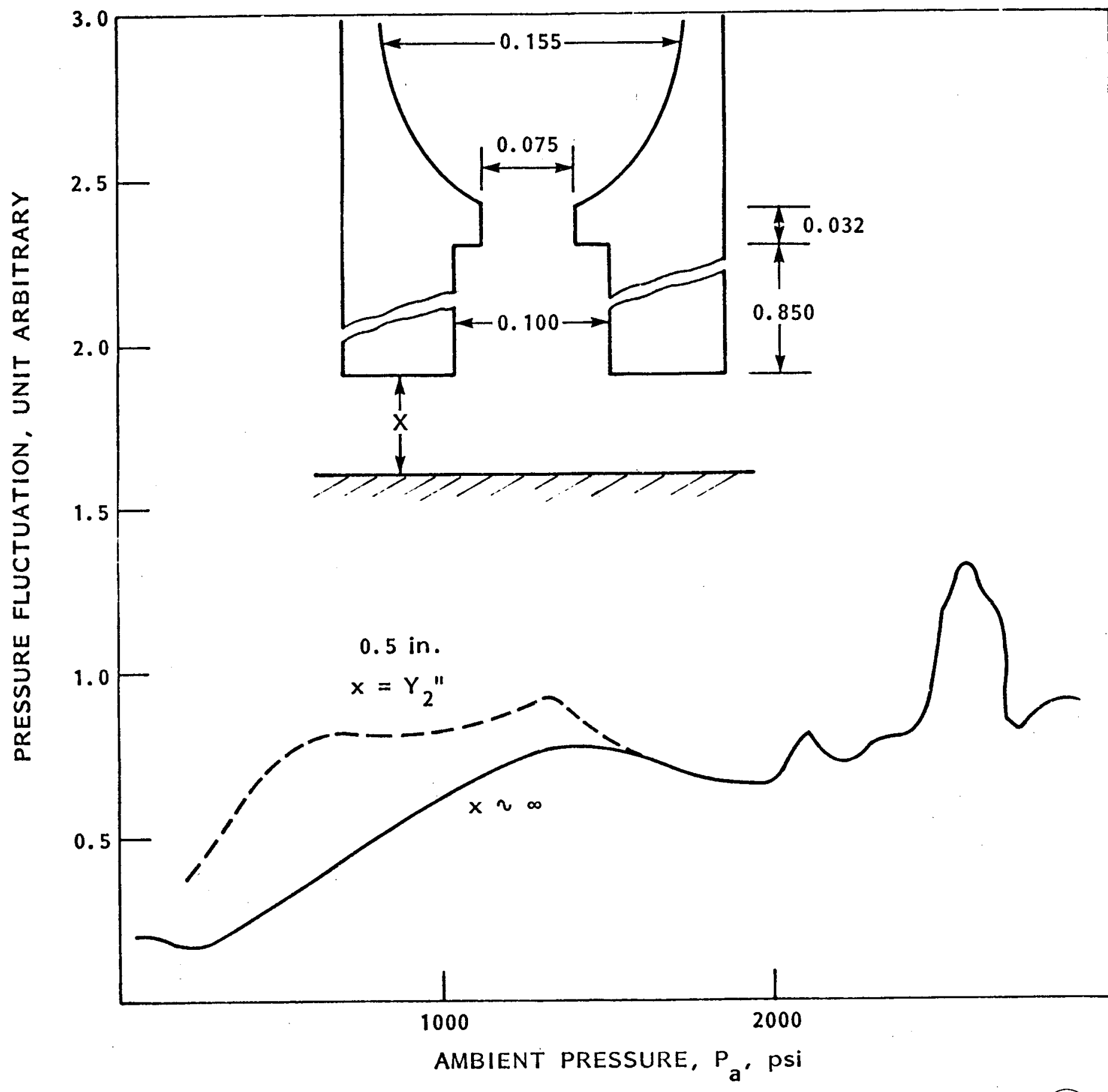

FIGURE 46 - INFLUENCE OF THE STAND-OFF DISTANCE, $X$, ON THE PRESSURE FLUCTUATIONS GENERATED BY A LONG ORIFICE NOZZLE AT LOW CAVITATION NUMBERS. $\triangle P \sim 6000$ psi 


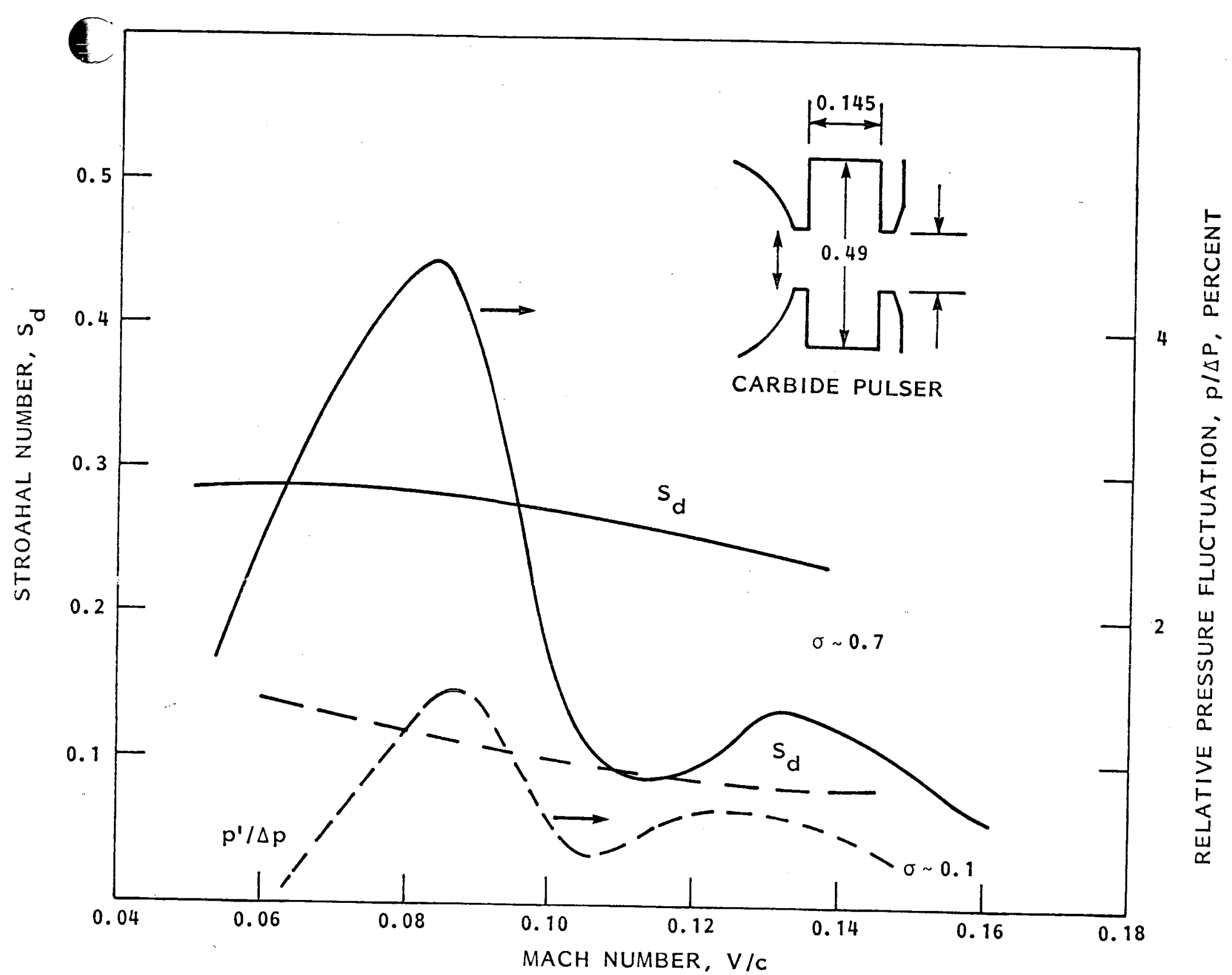

FIGURE 47 - PRESSURE FLUCTUATION MEASURED IN FEED PIPE AND STROUHAL NUMBER VARIATIONS WITH THE JET MACH NUMBER. PULSER-FED JET $d \simeq 0.10$ inch 


\section{Tracor Hydronautics}
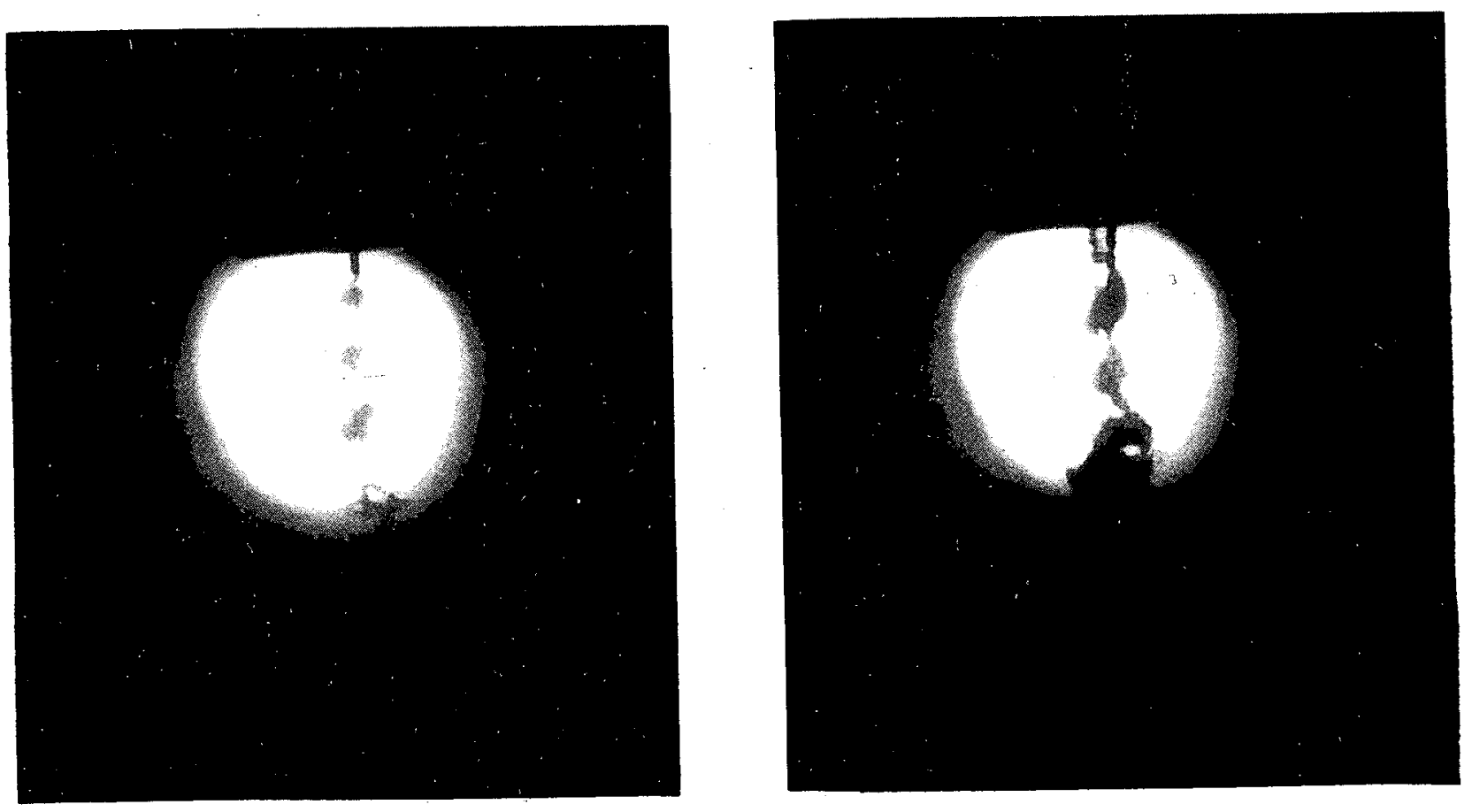

$$
\begin{aligned}
& \Delta P \simeq 1220 \mathrm{psi}, \quad \sigma=0.10 \\
& f=4.5 \mathrm{KHz}, \quad S_{d} \simeq 0.07
\end{aligned}
$$

$$
\begin{array}{ll}
\Delta P \simeq 565 \mathrm{psi}, & \sigma \simeq 0.06 \\
f \simeq 3.4 \mathrm{KHz}, & S_{d} \simeq 0.08
\end{array}
$$

FIGURE 48 - ASPECT OF CAVITATING JET PRODUCED WITH A PULSER-FED NOZZLE AT LOW CAVITATION NUMBER, $\sigma$ 
$-141-$

APPENDIX

Programs Listirgs 
$-142-$

Pipe Oscillations Program 
PFOGRAM FIFEES



IMFLICIT IIDUBLE FRECISION ( $A-H, L, O-Z$ )

FEAL*8 L1,L2,L3,LL3

COMPLEX*16 S,ZMIN

COMFLEX 16 GAMA1, GAMA2, GAMA3, SOFT, CF1, CF2, CF3, CN1, CH2, CN3

COMFLEX 16 ZC1, ZC2, ZC $3, Z D, Z U, Z C F, H U, Q U, Z X, H X, Q X, C S I N, C C O S$

COMFLEX*16 CF, CN, HU3, RUZ,HU2, QUS, HU1, QU1, ZC, GAMA

COMFLEX*16 CCOS1, CSIN1, CCOS2, CSTM2, CCOS3, CSTNZ

COMFLEX*16 QU,HU

DIMENSION OMEGA(200), COZF (200), OMEMIN(50), ZMIN $(50)$,

1 ASA $(200)$

COMMON/GEOM/F1, $R 2, F 3, L 1, L 2, L 3, A 1, A 2, A 3, C, F H O, Z V$

COMMON/CNST/DELTA,FMIN

COMMON/COMF1/GAMA1, GAMA2, GAMA3, CF 1, CF $2, C F 3, C N 1, C N 2, C N 3$

COMMON/COMF2/ZO,ZC1,ZC2,ZCZ

COMMON/FLOW/UJ,AJ

**********************************************************

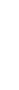

L.2

L.3 $1 \mathrm{nj}$ 
THIS FROGRAM COMFUTES THE UALUE OF COMFLEX S IIETERMINING OSCILLATIONS IN A FIFE WITH 3 STEFS

$C \quad=$ SFEEII OF SOUNII

F, ALFA $=$ FFICTION ANI FOWEF FACTOFS FOF FIFES

LI, III = LENGTH AND DIAMETER OF FIFE I

iIJ = JET MIAMETEK

UJ = JET VELOCITY

NFOIN = TOTAL NUMBEF OF INCFEMENTS FOF THE FREQUEMCY, OMEGA

DELTA = INCFEMENT OF OMEGA

FMIN = MIN. ACCEFTEI VALUE OF $F=Z E F I O$

ZCI = GAMMA' (S*AT)

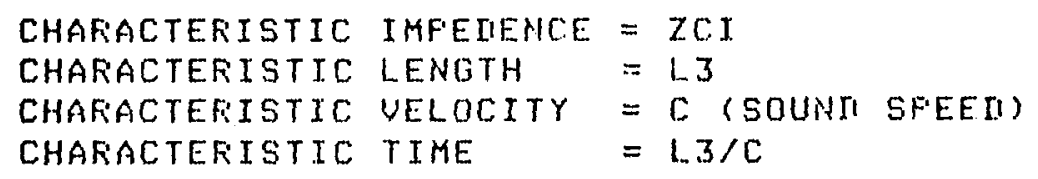

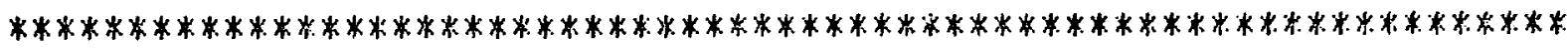

CALL GETCFU(NCHA1)

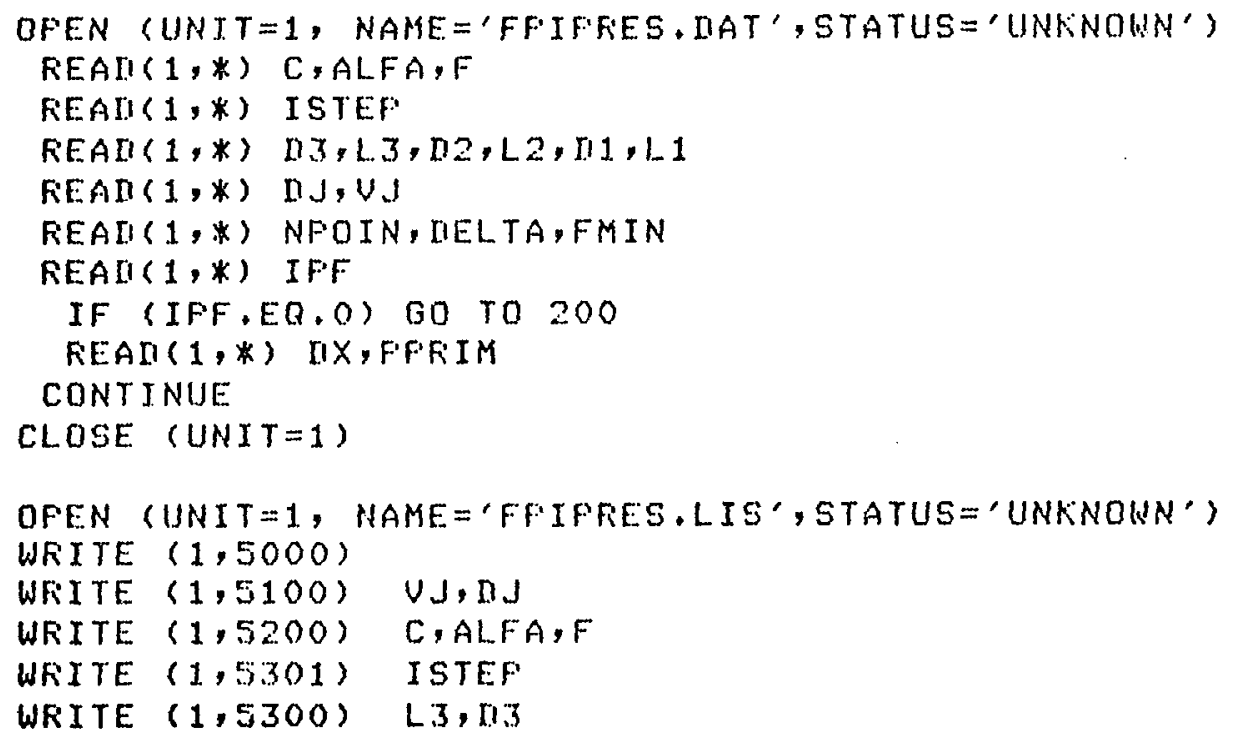




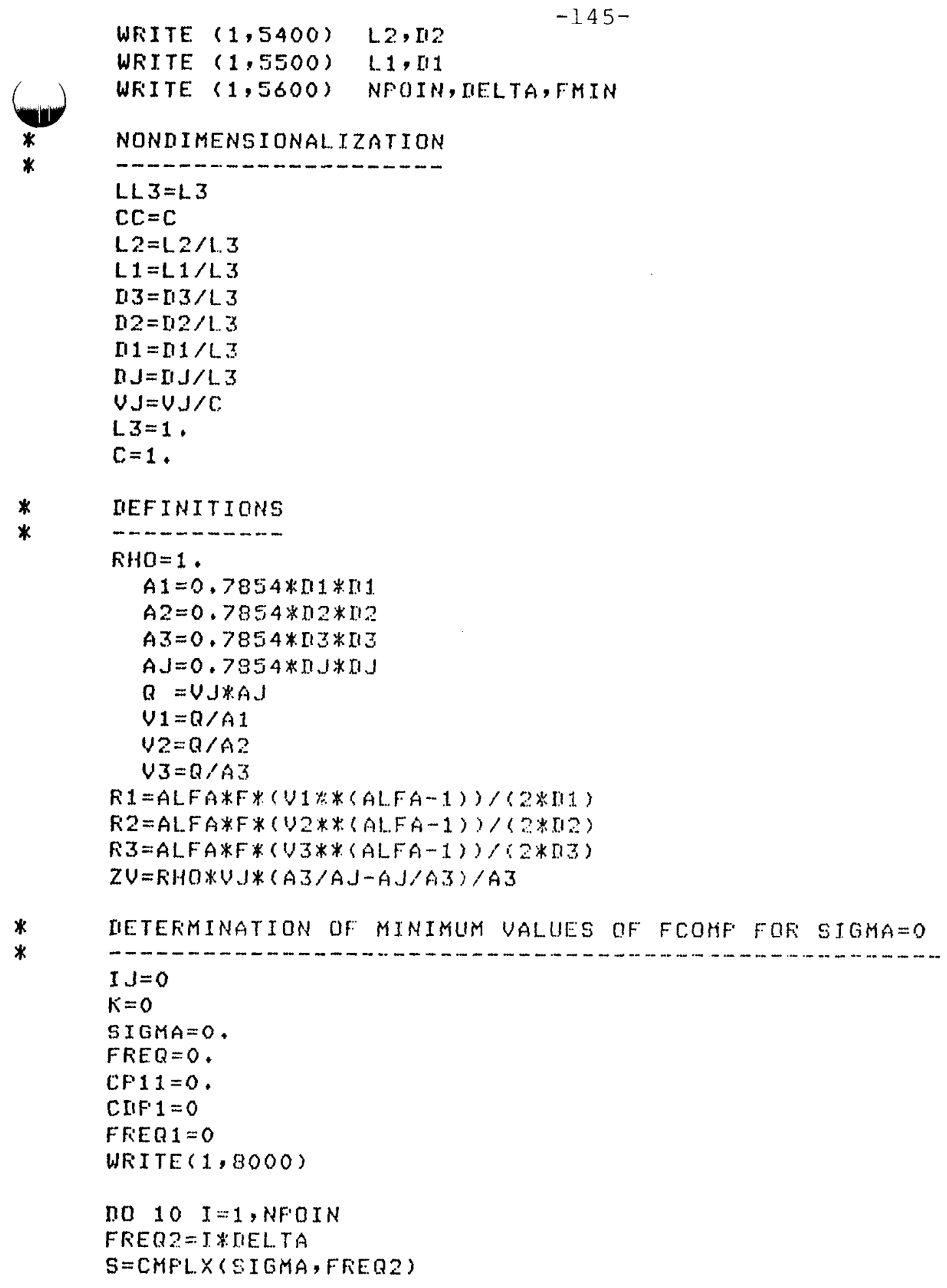

CALL ZF $(S, Z C F)$

$C F 22=\operatorname{CHABS}(Z C F)$

IF $(M O H(I, 5 O)+N E, O)$ GO TO 3

$I J=I J+1$

$\operatorname{OMEGA}(I J)=F R E Q 2$

$\operatorname{COZF}(I J)=C F 22$

$A S A(I J)=S I G M A$

WRITE $(1,1000)$ I J, SIGMA,FFEQ2, ZCF, CF22

$3 \quad C M P 2=C F 22-C P 11$

IF (CIF') $6,6,4$

$4 \operatorname{IF}(C I F 1) 5,5,6$ 
5 IF $(I, L T .2)$ GO TO 6

$K=K+1$

IF $(K, G T, 15)$ GO TO 30

$\operatorname{OMEMIN}(K)=F R E Q 1$

$Z M I N(K)=Z C F$

$6 \quad C P 11=C F 22$

C.IIF. $1=C I N F \cdot 2$

FRER1 $=F R E Q 2$

10 CONTINUE

30 CONTINUE

I JMAX $=I J$

OFEN (UNIT=2, NAME='ASIG, IIAT', STATUS='UNKHOWN')

WRITE $(2,1001)$ (ASA $(I), I J=1$, I IHAX)

CLOSE (UNIT $=2$ )

OFEN (UNIT $Y=2$, WAME = 'AOME, IAT', STATUS=' UNKNOWN')

WRITE $(2,1001)$ (OMEGA $(I J), I J=1, I J M A X)$

CLOSE (UNIT $=2$ )

OFEN (UNIT $=2$, MAME='AFUN, IIAT', STATUS='UNKNOWN')

WFITE $(2,1001)$ (COZF (IJ),IJ=1,I.JMAX)

CLOSE (UNIT $=2$ )

1001 FOFMAT (SE15.6,1X,'\&')

$K M A X=K$

WFITE $(1,7000)$ I JMAX, KMAX

IF (KHAX,EQ.O) GO TO 20

WFITE $(1,9000)$

WRITE $(1,2000)$ (OMEMIN(I), ZMIN(I), I=1, KMAX)

*

CORFECTION OF S

*

DO $20 \quad I=1, K M A X$

PULS1 $1=$ OMEMIN(I)

$Z F 1=Z M I M(I)$

$S=C M F L X(S I G M A, F U L S I)$

CALL OFTMS(FULS1, ZF1, SOFT)

PULS2 $=$ IIIMAG $S S O F T$ \}

$F=F \cup L 2 / 6.2332$

$F 1=F * C C * 12, / 1.13$

$F 2=F *(L 1+L 2+L 3) / L 3$

WFITE $(1,7001) F, F_{1}, F_{2}$

IF (IFF,EQ.O) GO TO 20

*

COMFUTE THE MONE SHAFE, SIX MODES AFE COMFUTEN

$I G K=M I N O(G, K M A X)$

IF (I,LE, IGK) CALL MSHAFE (I, SOFT, IIX,FFFIM)

20 CONTINUE

201 WRITE $(1,5001)$

1000 FORMAT $(12 X, I 5,5 X, 5(F 12,5,5 X))$ 
FOFMAT (12X, 3F16.5)

5000 FOFMAT $(/, 15 X$, 'CALCULATION OF FFESSUFE OSCILLATION IN FIFES', $/ /$ )

TO FORMAT $(/, 15 X, '$ JET VELOCITY = 'F12.3,2X,'FT/SEC', /, $15 X$,

1

' JET IIIAMETER = 'F12,3,2X,'INCHE.S')

5200 FOKMAT (/,15X,'SOUNI SFEEI',12X,'=',F13.5,2X,'FT/SEC',/,15X,

1 'PIPE FOUEE FACTOESALTA $=1,712.5 \%, 315 X$,

2 'PIFE FFICTION FACTOR, F = ,F12.5)

5301 FORMAT (/,10X, 'NUMEEF OF STEFS IN THE FIFE SYSTEM $=$ ', I3)

5300 FORMAT $(/, 10 X, ' F I P E$ NO. 3 = LAST DNE BFFOFE NOZZLE', $/ 1$,

1 10X, 'LENGTH OF FIFE $3, \quad L 3=1, F 9.5,2 X,{ }^{\prime}$ INCHES', $5 X$,

2 'DIAMETER OF FIFE $3, \mathrm{I3}=$ ',F9.5, 2X, 'INCHES')

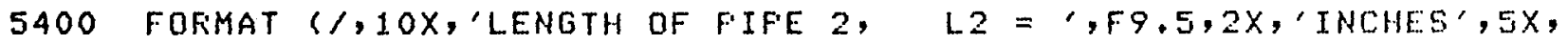

1 'IIAMETEF OF FIFE $2, \square 2=$ 'F9,5,2X,'TNCHES')

5500 FOFMAT $(/, 10 X$, 'LENGTH OF FIFE 1, L1 = ',F9.5,2X, 'INCHES', $5 X$,

1 'DIAMETER OF FIFE 1 , II1 = ',F9.5,2X,' INCHES',

5600 FOFMAT $/ /, 15 X$, 'TOTAL NUMEEF OF INCFEMENT USEI FOF THE',

1. $1 X$, 'FFEQUENCY CALCULATION, NFOIY $=1,15,1,15 X$,

2 'INCFEMENT OF FFEQUENCY USED, DIELTA $=$, FB, $5.115 X$,

3 'MINIMUM ACCEFTEI UALUE OF F=ZERO USEU, FMIN $=$ ',FB.5, 11 ,

5001 FORMAT (//,',***ENI OF COMFUTATION***')

7000 FOFMAT $(/, 12 X, '$ I JMAX $=$ ', I $4,5 X, '$ KMAX $=$, , I $1, /$ )

7001 FOFMAT(10X,'THIS COFFESFONIS TO THE FFEQUENCY ', F6.3,'*C./L3 ='

1,F12.5, $5 X$, 'LAMNA/L= ',F12,5,/1)

8000 FOFMAT///,16X,'I', 11X,'SIGMA', $11 X$, 'OMEGA', $18 X$, 'F COMFLEX',

$\left.120 X,{ }^{\prime} I F I^{\prime}, 1\right)$

9000 FORMAT(//19X,'OMEGA MIN', $16 X, ' Z$ MIN COMFLEX')

CALL GETCFU(NCHA2)

CHATOT $=($ NCHA2 $-N C H A 1) / 100$.

TIMETOT $=$ CHATOT/SO.

WHITE $(1.1004)$ TIMETOT, CHATOT

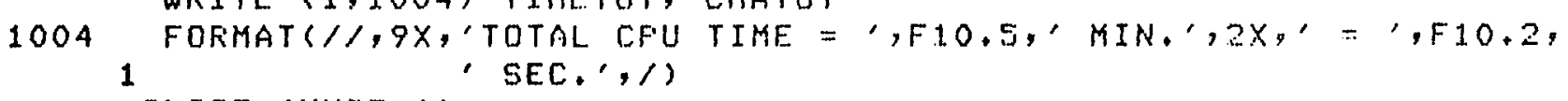

1

CLOSE (UNIT $=1$ )

STOF

END

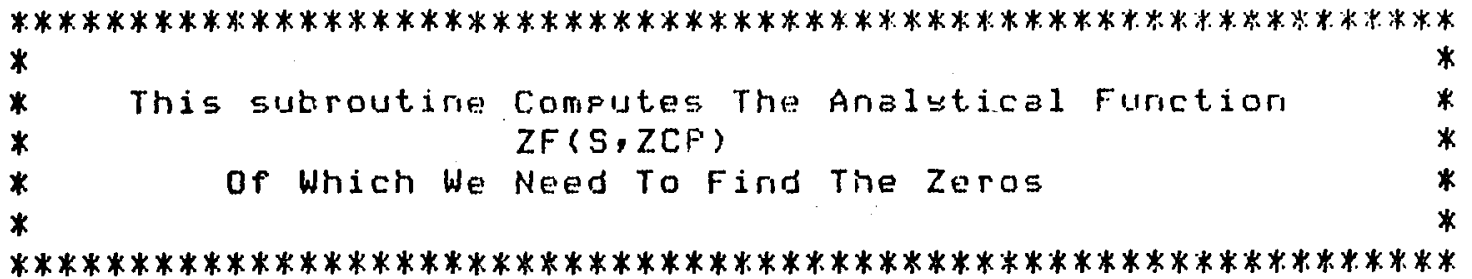

SUBROUTINE ZF (S,ZCF)

IMFLICIT TIOUBLE FRECISION $(A-H, L, O-Z)$

REAL*8 L1,L2,L3,LL 3

COMFLEX*16 GAMA1, GAMA2, GAMA3, SOFT, CF1, CF2, CF3, CH1, CN2, CN3

COMFLEX 16 ZC1,ZC2, ZC3,ZO,ZU,ZCF,HU, RU,ZX, HX, QX, CSIN,CCOS

COMFLEX*16 CP, CN, HU3, QU3, HU2, QU2, HU1, QU1, ZC, GAMA

COMPLEX*16 CCOS1, CSIN1, CoOS2, CSIN2, CCOS3, CSIN3

COMFLEX*16 S,TO1,T02,T03,FA,FE,FC

COMPLEX*16 QU,HU 
GAMA1 = CISQRT $(S * S+R 1 * S) / C$ GAMA2 $=$ CISQRT $T(S * S+R 2 * S) / C$ GAMA3 =CIISRRT $(S * S+F 3 * S S) / C$

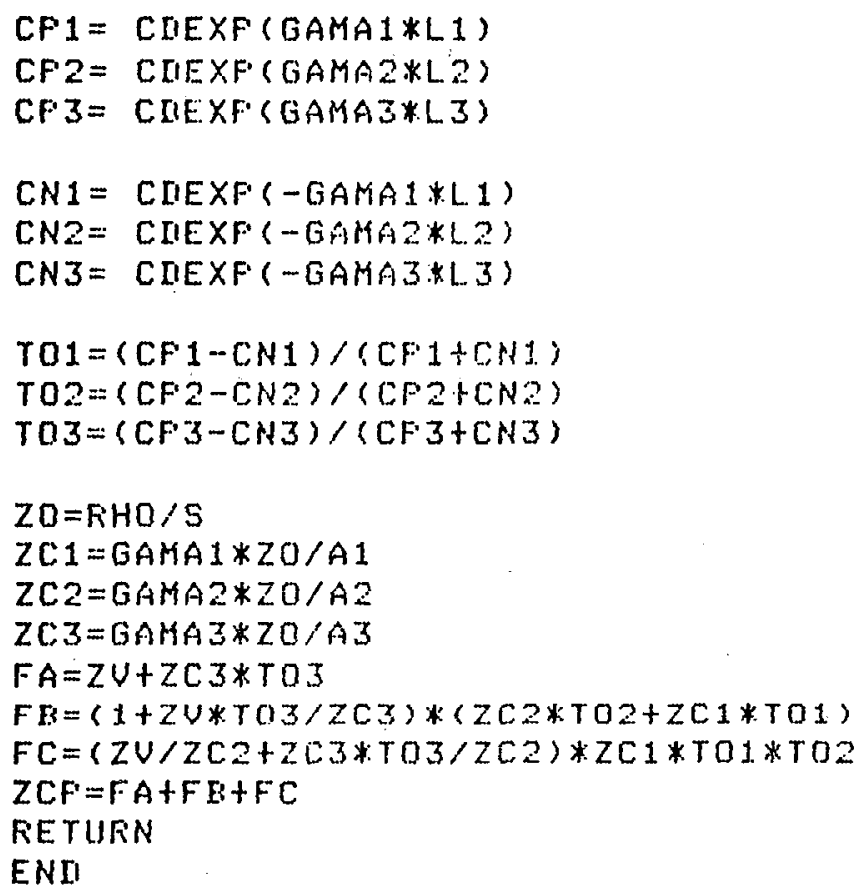


$E F S=C M F L X(I E L, I I E L)$

$s)_{S=S+E F S}$

NLOOF'=HLOOF'+1

CALL ZF $(S, Z C F)$

$Z F 2=Z C F$
$Z Z F F=Z F 2-Z F 1$

$Z Z F F 1=(1 E-10, \triangle E-10)$

IF (IIABS (IIFEAL (ZZFF)),LT, IE-10, ANI, IIABS(IIMAG(ZZFF))

1

DLTAS $=-E F S * Z F 1 / Z Z F F$

$S=S+I I L T A S$

SIGMA $=$ IFEAL (S)

FULS2 $=$ UIMAG $\{S\}$

IIFULS = IABS (FULS2-FUL)

CALL ZF $(S, Z C F)$

$A Z F=C D A E S(Z C F)$

WRITE $(1,1000)$ S,AZF

IF (IFULS, GT, I, G GO TO 9

IF (AZF,LT,FMIN) 60 TO 10

IF (NLOOF,EQ.5) DO TO 11

CALL ZF $(S, Z C F)$

$Z F 1=Z C P$

GO TO 5

$9 \quad S=C M F L X$ (SIGHA, FUL)

WRITE $(1,6000) \quad S$

CALL ZF $\left(S, Z C F^{*}\right)$

$\mathrm{ZF} 1=\mathrm{ZCF}$

GD TO 5

10 SOPT $=S$

AMFL $=-F \cup L S 2 /(2, * S I G M A)$

WRITE $(1,4000)$ SOFT, AZF, NLOOF ,AMFL

GO TO 12

11 CONTINUE

$E F S=E F S / 10$

$N L O O F=0$

GO TO 5

12 CONTINUE

C

1000 FOFHAT $\left(15 X, ' S=\left(', 2 F 12.5,{ }^{\prime}\right), 5 X, ' A Z F=,, F 12.5\right)$

2000 FOFMAT $15 X$, 'NO ZERO FOF ZF NEAF THIS UALUE OF OMEBA AFTEF 10 ITEFA 1 TIONS', 15X, 'AMFLIFICATION $=,, F 7.2,1 / 1)$

3000 FORMAT(//.5X,'OMEGA $=$, FB.5, $\left.1,5 \times, 16\left({ }^{\prime} *^{\prime}\right), 1\right)$

4O( FOFMAT (/5X,'SOFT = (', 2FJ2.5,')',5X,'AZFNUL=:, F12.5,10X,'ILQOF=',

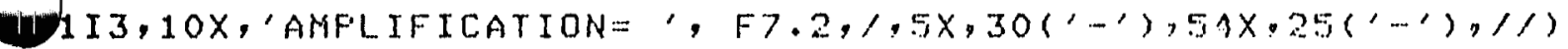

6000 FORMAT(//,15X, 'TOO FIG JUMF IN OMEGA',5X, 'HEW STAFT AT $5=(', 2 F 12$. $15, \cdot)(1 /)$ 
SUEROUTINE MSHAFE (IJ, SOFT, IIX,FFRIM)

IMFLICIT IIOUELE FRECISION $(A-H, L, O-Z)$

COMFLEX*16 GAMA1, GAMA2, GAMA3, SOFT, CF1, CF2, CF3, CN1, CH2, CN3 COMFLEX 16 ZC1,ZC2,ZC3,ZO,ZU,ZCF, HU, QU,ZX,HX,QX,CSIN,CCOS COMFLEX*16 CF, CN, HU13, QU3, HU2, QU2, HU1, QU1, ZC, GAMA COMPLEX*16 CCOS1, CSIN1, CCOS2, CSIM2, CCOS3, CSTN3 COMFLEX*16 RU, HIS

COMMON/GEOM/FI, R2, FI, L1, L2,LZ,A1,A2,A3,C, RHD, ZU COMMON/COMF1/GAMA1, GAMA2, GAMA3, CF1, CF2, CF3, CN1, CN2, CN3 COMMON/COMF $2 / Z O, Z C 1, Z C 2, Z C Z$

COMMON/FLOW/UJ,AJ

DIMENSION ZA $(600,6)$, HA $(600,6)$, RA $(600,6)$, FHASEZ $(600,6)$, 1 FHASEH $(600,6)$, FHASEQ $(600,6), X(600), Z \times(600)$

REAL*B L1,L2,L3,LL3

CHARACTEREB FZA

DATA FZA/'FZA . IIAT',

CHARACTEF* FHA

DATA FHAI'FHA , MAT',

CHAFIACTEFIEB FQA

DATA FRA/'FQA . DAT',

CHAFACTERT 11 FFHZ

DATA FFHZ,'FHASEZ. DAST',

CHARACTERE 11 FFIHH

MATA FFHH/ 'FHASEH , MAT',

CHARACTER 11 FFHQ

DATA FFHQ/ 'FHASEQ WAT'/

CALL ZF (SOFT,ZCF)

WR I TE $(1,1300)$

1300 FORMAT( $/ 1,15 X$, 'FRESSURE ANI FLOW COMFUTATION INSIIEE THE FIFE' $\left.\left.1, /, 15 \times, 45 i^{\prime} *^{\prime}\right), / /\right)$

WRITE $(1,1200)$ I J, SOFT, ZCF

1200 FORMAT $/ /, 15 X, '$ COMFUTATION FOF MSHAFE AT MOLE = "I2,'SOFT: $"$, $12 E 15.5,{ }^{\prime}, \prime, /, 40 X,{ }^{\prime}$ ZCP $\left.=\left({ }^{\prime}, 2 E 15,5,{ }^{\prime}\right)^{\prime}, / /\right)$

$C \cos 1=(C P 1+C N 1) / 2$.

$\operatorname{CSIN1}=(\operatorname{CF} 1-\operatorname{CN} 1) / 2$.

$\operatorname{CCOS} 2=(C F 2+C N 2) / 2$.

$\operatorname{CSIN} 2=(\operatorname{CF} 2-\operatorname{CN} 2) / 2$.

$\operatorname{CCOS} 3=(C F 3+\operatorname{CN} 3) / 2$.

CSINZ $=(C F 3-C N 3) / 2$.

* comfutation of hu1, Qui, hu2, Qu2, huz, QUz

$H U=P F R I M$ 
QU $=F \cdot F \cdot R I M / Z V$

HUZ $=H U * \operatorname{cocos} 3+Z \operatorname{Co} 3 * Q \cup * \csc N 3$

QU3 $=H U * \operatorname{CSIN} 3 / Z \operatorname{CB}+Q U * \operatorname{cc} \cos 3$

HU2 $=H U 3 * \operatorname{CCOS} 2+Z C 2 * Q U 3 * C S I N 2$

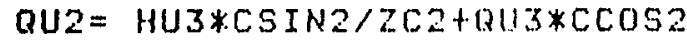

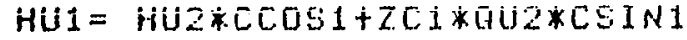

QU1 $=\operatorname{HU2} * \operatorname{CSIN1} / Z C 1+Q U 2 * \operatorname{CCOS} 1$

* initialization

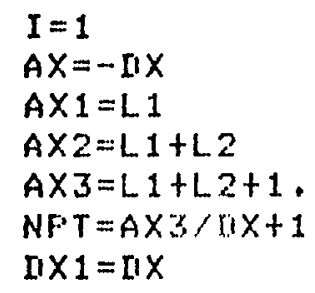

101

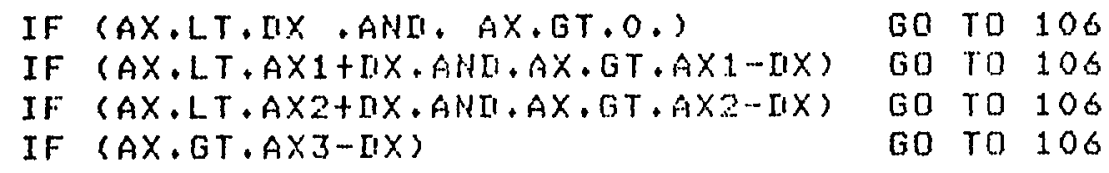

103 IF $(A X, G T \cdot(A X 3+N X))$ G0 T0 104 GAMA $=$ GAMA3

Z.C $=\mathrm{ZCS}$

$H U=H U$

$\mathrm{QU}=\mathrm{QU}$

$A L T=A \times 3$

\section{CONTINUE}

$C F=C N E X F($ GAMA* $(A L T-A X))$

$C N=C I E X P(-G A M A X(A L . T-A X))$

$\operatorname{CCOS}=(C F+C N) / 2$. 
$\operatorname{CSIN}=(C F-C N) / 2$

$H X=H U * \operatorname{coc} \theta+Z C * Q U * \operatorname{cs} I H$

$Q X=H U * \operatorname{csI} I / Z C+Q U * \operatorname{ccos}$

$Z X(I)=H X / Q X$

$Z A(I ; I J)=C \operatorname{PABS}(Z X(I))$

$\operatorname{PHASEZ}(I, I J)=A \operatorname{TANII}(\operatorname{IIMAG}(Z X(I)) / F E A L(Z X(I)))$

$H A(I, I J)=C$ IIAES $(H X)$

$\operatorname{PHASEH}(I, I J)=A T A N D(I I M A G(H X) / F E A L(H X))$

$R A(I, I J)=C[A B S(R X) * U] / A J$

PHASEQ $(I, I J)=A T A H I M(I I M A G(Q X) /$ REAL $(Q X))$

$$
\begin{array}{llll}
I=I+1 \\
I F(I, G T, N F T) & \text { GO TO } & 104 \\
\text { IF }(A X, L E, A X 3) & \text { GO TO } 101
\end{array}
$$

104 OFEN (UHIT =10, NAME='F'XHQ. IAT', STATUS= 'UNKNOWN')

WRITE $(10,1000)(X(I), I=1, N F T)$

CLOSE (UNIT $=10$ )

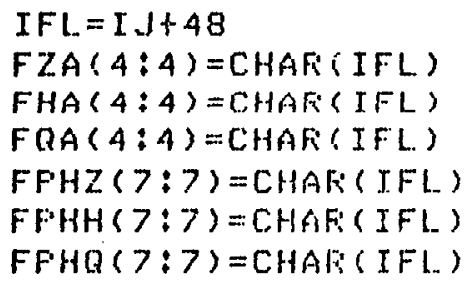




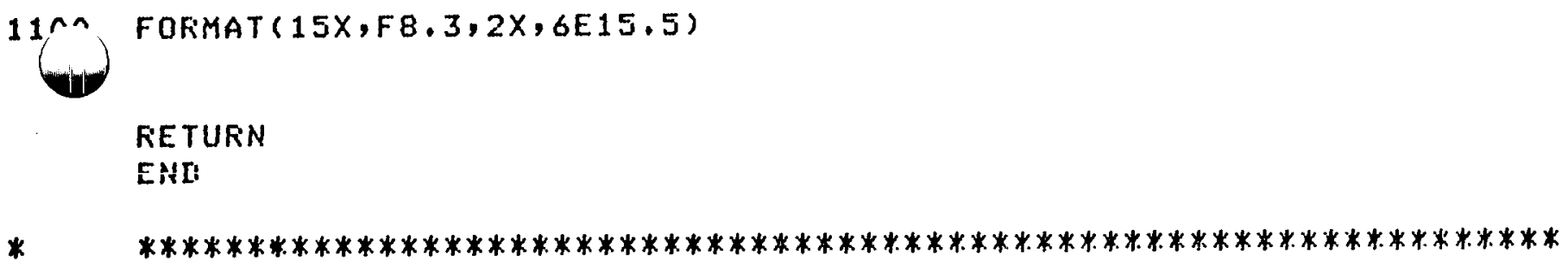


$-154-$

Bubble Ring Collapse Program 
Tracor Hydronautics

FROGRAM INEIN10

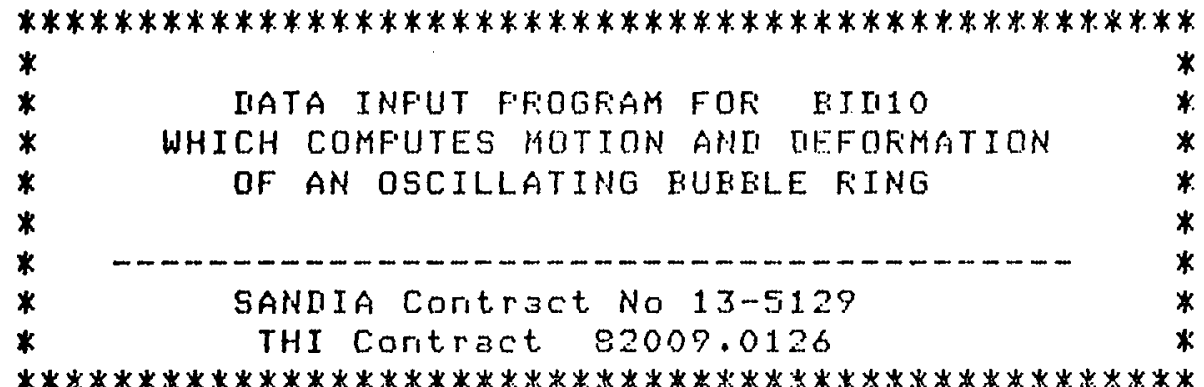

DIMENSION AUXI(10)

OPEN (UNIT = 6, NAME ='IHII110.LIS', STATUS='UNKHOWN')

9 TYFE 10

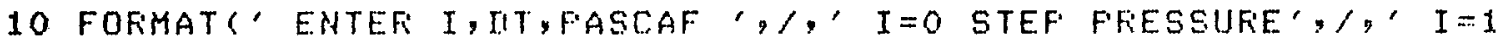

1. FOF FRESSUFE IIROF OF IUUFTION IIT', $/$, I 2 SINUS FFESSUFE',

1' $n T=$ LAFS OF TTHE IN FFESSUFE TIFOF OFTION ',

1. PRESSURE RECOUERY TO FASCAF = , 来)

REAI $(5, *, E R F=9)$ I, IT, FASCAF

80 TYFE 81

81 FOFMATく' ENTER AMFLITUIE ANI FFEQUENCY OF FFESSURE FUNCTION', /,

1 'FAMF, FFEQ: ', 束)

REAI $(5, *, E R F=80)$ FAMF, FFER

ENII IF

50 TYFE 51.

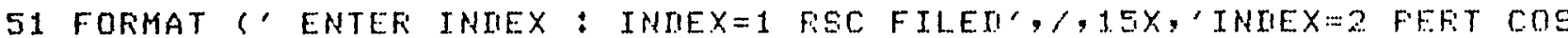

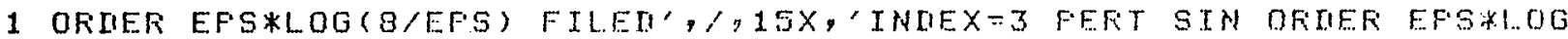

1 (8/EFS) FILEI', /,15X,'INDEX=4 FEFT COS OFIER EFS FTLEL', / ISX,'IHII

$1 E X=5$ FERT SIN OFIIER EFS FILED', \$)

REAI $(5, *, E F i=50)$ INIIEX

60 TYPE 61

61 FOFMAT(' ENYEF IOFT, IOFT = 1 NCAS FGAZ', ,', IOFT= O

1 NCAS CIFC, $\$$ )

REAI $(5, *, E F F=60)$ IOF.T

IF (IOFT,EQ+1) THEN

11 TYFE 12

12 FOFHAT(' ENTEF EFS,W,EK, CIFIC , CHAFACTERISTIC OF TORUS',

KIEAN $(5, *, E F R=11)$ EPS, W, EK, AUXIO

ELSE

65 TYF.E 66

66 FOFMAT(' ENTEF: EFS,W,EK,FGZO, CHAFACTEFISTIC, OF TOFUS', $\$$

FEAD (5;,$E R R=65)$ EPS,W,EKI, AU:IO

ENII IF

3 TYFE 14

14 FOFIAT (' ENTEF TINT, STEF OF TIME',\$)

FIEAII $(5 * *, E F I T=13)$ TINT

15 TYFE 16

16 FOFMAT(" ENTER NCAS, NUMBER OF CASES',\$)

READ $(5, *, E F R=15)$ NCAS 


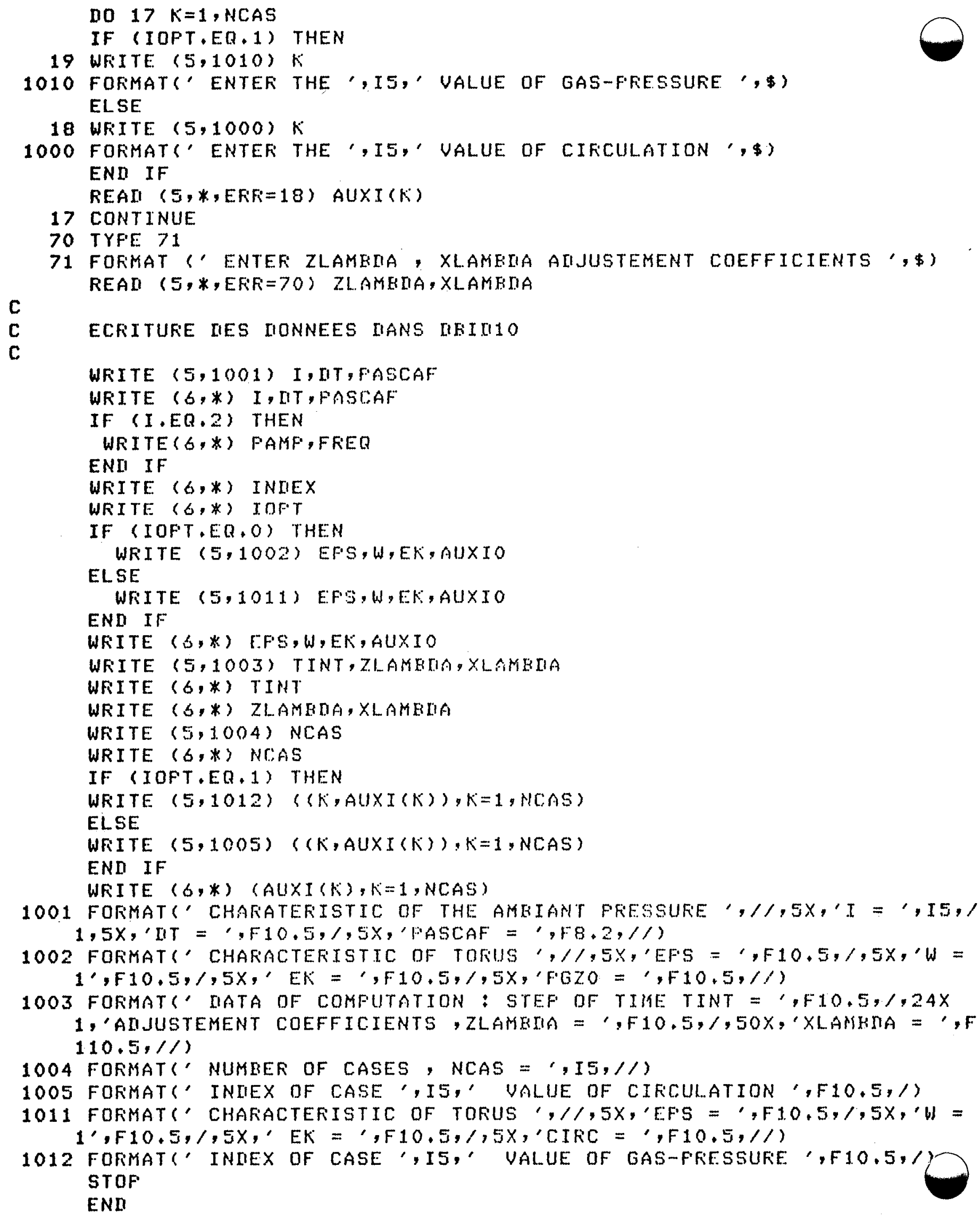


Tracor Hydronautics

PROGRAM RIM10

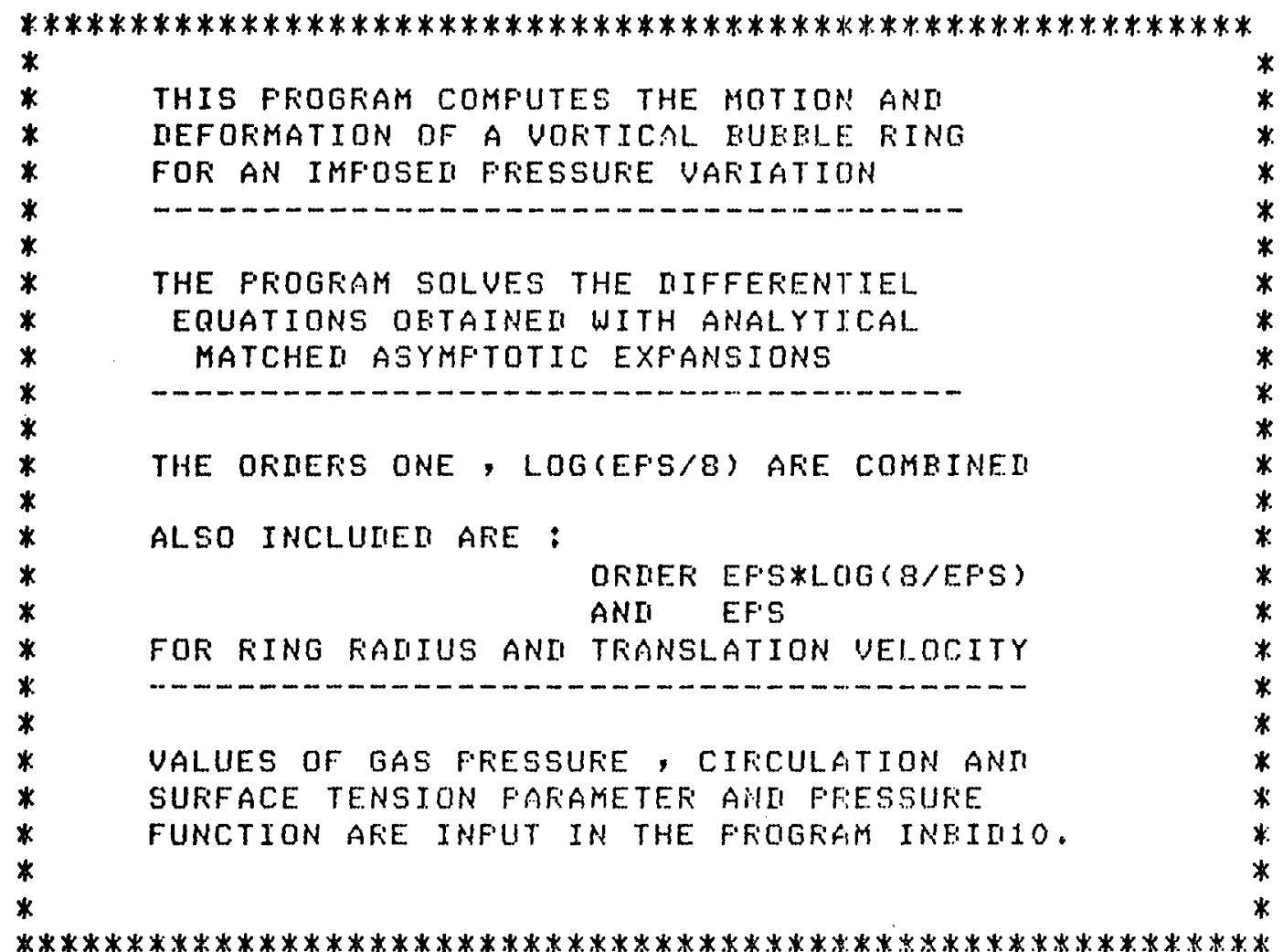

EXTERNAL FICSEC,FASCAL

DIMENSION AUXI(10), TIME (501), FESUL (501), XIES(5)

COMMON/FHIL/I, FAMF, ITT, FASCAF, FREQ

COMMON/IEFEN/EFS, F, W, EK, FGZO, CIRC, TAUB

COMMON/TESUF /WB

CREATION DF FILE IIATA

CHAFACTEFET, FFE

DATA FRE/'REE . DAT',

CHARACTEET 7 , FRT

DATA FRT/'RT . IIAT'/

DPEN (UNIT=G, NAME=' DBID10.LIS', STATUS='UNKNOUN')

OPEN (UNIT =2,NAME='FBII1O.LIS', STATUS='UNKNOWN')

(

DATA INFUT

READ $(6, *)$ I, DT, FASCAF

IF (I,EQ, 2) THEN

READ(G,*) FAMP, FREQ

END IF 


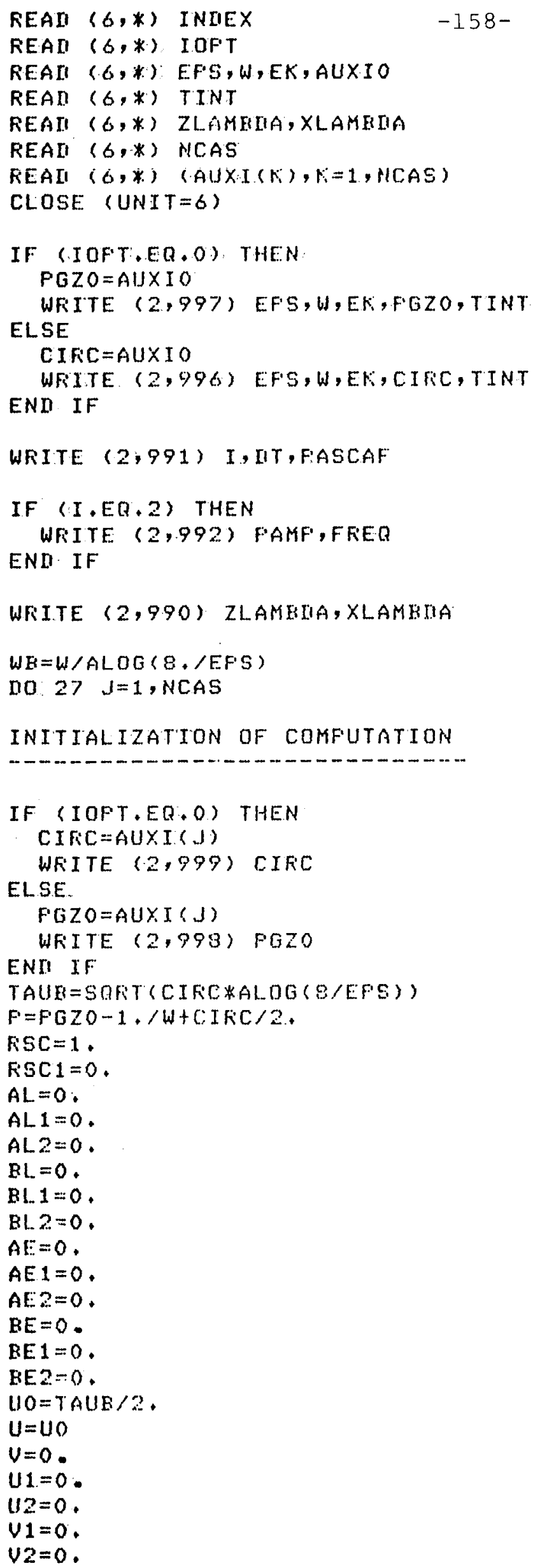


UEO $=-1$. *TAUE $/ 4,+1, / W E / T A U B / 2$.

UE $=$ UEO

$U E=0$.

UE $1=0$.

$\triangle E 2=0$.

VE $\hat{1}=0$.

VE2 $=0$.

$Z 1=E F S *(A L .06(8, / E F S) *(1,+1 . / W / T A U B / T A U E)-.5) * T A L L / 2$.

$Z 2=Z 1$

$Z=0$.

ROND1 $=0$.

$\operatorname{RON} 12=0$.

RONII=1.

$\mathrm{T}=0$,

$N N=1$

no $10 \quad M M=1,10001$

$M=M M-1$.

FSI $=F A S C A L(T)$

COMFUTATION OF IIEFOFMATIONS OF OFIEFS EFSWLOG ( $8 / E F$ S) AND EFS COMETNEI

$A L=A L+(3, * A L 1-A L 2) * T I N T / 2$.

$A E=A E+(3, * A E 1-A E 2) * T I N T / 2$.

$\mathrm{EL}=\mathrm{BL}+(3, * B L 1-\mathrm{EL} 2) * T \mathrm{~T} M T / 2$.

$B E=E E+(3, * B E 1-\mathrm{KE} 2) * T \mathrm{INT} / 2$.

$A L 2=A L 1$.

$A E 2=A E 1$

AL. $1=Z$ ZLAMEMA* $(U-U O)$

$A E 1=Z L A M B[I A *(U E-U E O)$

$B L 2=B L 1$

$\mathrm{BE} 2=\mathrm{BE} 1$

BLI $1=X L A M E M A * V$

BE $1=X$ LAMBIA

COMFUTATION OF RADIUS, RRONA, ANO MOTION IN THE $Z$ IIJFECTION

RONII=FONII+ (3 * *RONIII-FONIZ) *TINT $/ 2$,

RROND = (RONI1-1 ) /EFS/ALOG (.$/ E F S)$

$U \times 1=(1,-X$ LAMEIIA $) * U$

$U \times 2=(1,-X$ LAME[IA $) * U E$

RONII $=$ FIONIII

RONII $1=E F S *(A L O G(3, / E F S) * U \times 1+U \times 2)$

$U X=R O N D 1 / E F S / A L O G(O$. $/ E F S)$

$Z=Z+(3, * Z 1-Z 2) * T$ INT $/ 2$.

$U Z 1=(1,-Z L A M B I A) *(U \cdots U O)+U O$

$U Z Z=(1,-Z$ LAMEIA $) *(U E-U E O)+U E O$

$Z 2=Z 1$

$Z 1=E F S *(A L O G(8, / E F S) * U Z 1+U Z 2)$

$V Z=Z 1 / E F S / A L O G(\Omega, / E F S)$

OUTFUT FRINTOUT

IF $(M O N(M, 20), N E, 0)$ GO TO 2

WRITE $(2,1000) T, F S C, U, U, U E, V E, A L, B L, A E, B E$

$T I M E(N N)=T$

$X \operatorname{DES}(1)=\mathrm{RGC}$

$X \operatorname{IIES}(2)=A L$ 
$X \operatorname{XIES}(3)=E L$

$X \operatorname{IES}(4)=A E$

$X \operatorname{RES}(5)=\mathrm{HE}$

RESUL $(N N)=X$ RIES $($ INIIEX)

IFT $=R N$

Hin=

* COMfUtation of $U, U 1, U 2, U, U 1, V 2, U E 1, U E 1, U E 2, V E 1, V E 1, V E 2, F i S C, R: S C 1$

2 CALL SYSUIF1 (U,U1,U2,U,U1, U2, RSC, FSC1, FCSEC, TINT, T)

CALL SYSUIF2 (UE, UE1, UE2, VE, VE1, VE2, FSC, RISC1, FCSEC, TIHT, T)

CALL KUTTA(KSC, FSC1, TINT, FICSEC,T)

IF (RSC,LE. O.) G0 TO 11

$T=T+T I N T$

10 CONTINUE

11 IFL $=\rfloor+48$

FRE $(3: 3)=$ CHAF $(I F L)$

OFEN (UNIT $=10+1$, NAME=FFE, STATUS= 'LINKNOUN')

WRITE $(10+J, 1200$ ) (FESUL (INN), INN=1, TFT)

CLOSE (UNIT $=10+\mathrm{J})$

FRT $(3: 3)=$ CHAF: (IFL)

DFEN (UNIT $=10+J$, NAME =FRT, STATUS= 'UNKNOWN')

WRITE $(10+J, 1200)$ (TIME (INH), INN=1, TFT)

CLOSE (UNTT $=10+J)$

\section{CONTINUE}

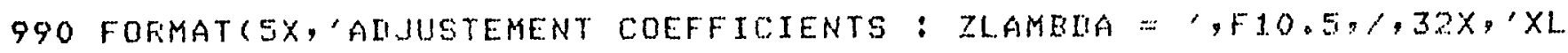
$1 M E I . A=(, F 10.5,1)$

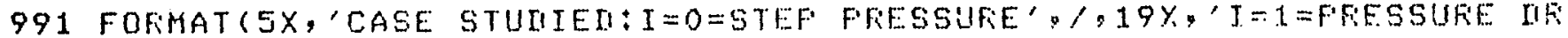
IFS THEN RECOUERS" $/, 19 X, ' T=2-F F E S$ SUEE VARTES AS SIN FUNCTION" $/$,

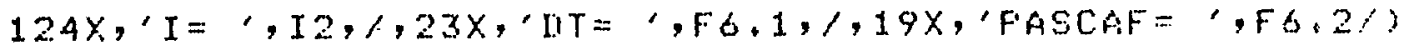

992 FORMAT(5X, 'AMFLITUUE OF THE FRESSURE: , F1.0.2, $1,5 X$,

1 'FFERUENCY OF THE FFESSUFIE = ',F $10,2, \prime)$

996 FORMAT (5X,'CHAFACTERISTIC OF TOFIUS : EFS $=$ 'F1.0.5, $1.30 X, ' W=$ $1 F 10.5, / 30 X, ' E K=', F 10.5,1,30 X, '$ CIFC $=, 9 F 10.5,1 / 5 X$, IIATA OF 1OMFUTATION : TINT $=, F 10.5,1)$

997 FOFMATS SX, 'CHARACTERISTIC OF TORUS : EFS = 'F10.5., $30 \mathrm{X}, ' \mathrm{~W}=$

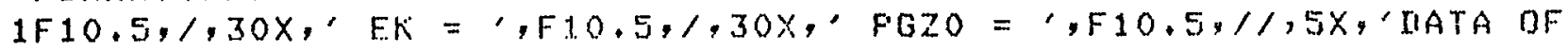
1OMFUTATION: TINT $=$, F10.5,/)

998 FORMAT $/, 10 X$, RESULTS FOF A GAS-FFESSURE FGZO = ,F10.5,1/,5X, T 1 IME', 6X,'RAIIUS RSC FEFT COS FEFT SIN FEFT COS FERT SIN 1 DEF COS DEF SIN DEF COS MEF SIN',/)

999 FORMAT $/, 10 X$, 'FESULTS FOF A CIFCULATION CIRC $=$,F10.5,/1,5X,'T 1 IME', $6 X$, 'RALIUS FSC FERT COS FERT SIN FERT COS FERT SIN 1 IIEF COS IIEF SIN DEF COS IIEF SIN, 1 )

1000 FORMAT $(2 X, F 10,5,2 X, F 10,5,2 X, F 10,5,2 X, F 10,5,2 X, F 10,5,2 X, F 10,5,2 X, F$ $10.5,2 X, F 10,5,2 X, F 10,4,2 X, F 10,4$ )

1200 FOFIMAT (SE15.6, $\left.1 . X,{ }^{\prime},{ }^{\prime}\right)$

STOF

ENII 
FUNCTION FICSEC $(X, X 1, T)$

****************************************************************** *

* function fielating the seconi ilefiluative of the * * radius to the fialius ani its fifist inefívative * *

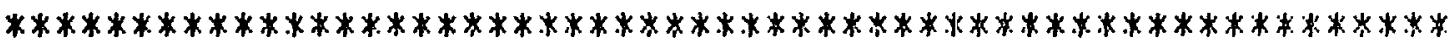

COMMON/FHIL/I, FAMF, IIT, FASCAF, FREQ

COKMON/BEKEN/EFS, F, U, EK,FGZO, CIRC, TAUB

RCSEC $=A L O G(B, / E F S) / A L O G(E F S / 8, * X) *(F A S C A L(T)+F *(1,-X * *(-2, * E K))$ $1+(1 . / X-X * *(-2, * E K)) / W-X 1 * X 1 *(A L O G(X * F F G, B)+, 5$,

$1 A L O G(B, / E F S)-C I F C *(1, / X / X-X * *(-2, * E K)) / 2,) / X$

RETURN

ENI 
* Tracor Hydronautics

FUNCTION FASCAL (TETA)

COMMON',FHILII, FAMF, ITT, FASCAF, FFER

****************************************************** $*$ * tiefinition of the ameient fressure function * * *******************************************************

$I=0$. STEF FUNCTION

$I=1$ - FRESSURE IIROF DF IUURATIDN DT RETURin to FFEsSURE value fiascaf

I=2 - SINUSOIXIAL FUNCTION FFEQUENCY FFEQ AMFLITUIE FAMF'

$K=I-1$

IF (K) $10,20,30$

10 F.ASCAL $=1$.

RETURN

20 IF (TETA-ITT) $1,21,21$

1 FASCAL $=-1$. RETUEN

21 FASCAL $=$ FASCAF RETUEN

30 FASCAL = (FAMF*COS (FFEQ $* T E T A)-1,) / 2$. FETURN

ENII 
* Tracor hyororieutics

SUEFIOUTINE SYSIIF $1(U, U 1, U 2, V, V 1, V 2, F, F 1, F C T, T I N T, T)$

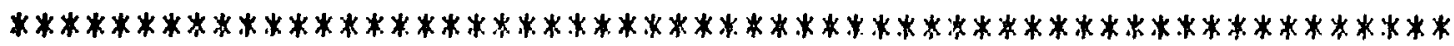
*

* this suefiqutire sol.ves the itffefientiel

* equations fofi the fiefitufirations of ofinefi * EFS*LOG(8/EFS) IN COSIME ANII SiNE

*

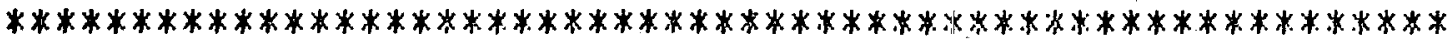

COMMON/BEFEH/EFS, F, W, EK, FGZO, CIFC, TAUE

$\mathrm{U}=\mathrm{U}+(3+* U 1-U 2) * \mathrm{~T} \mathrm{TNT} / 2$.

$U=U+(3, * U 1-U 2) * T I N T / 2$.

$U_{2}=U 1$

$v_{2}=v_{1}$

$U 1=(2, * T A U B *(F i 1-U / F)-2, * U * F i 1) / F$

$B S=-1$ * *TAUB*TAUR $/ R+(F * F C T(R, R 1, T)+2$ * *R $1 * R 1) * R$

$U_{1}=(B S+2$. W TAUB $* U / F-2$. *FI 1 WU $) / F$

RETURN

ENII 
SUEROUTINE SYSIIF2 (UE, UE1, UE2, VE, VE1, VE2, F, FI1, FCT, TINT, T)

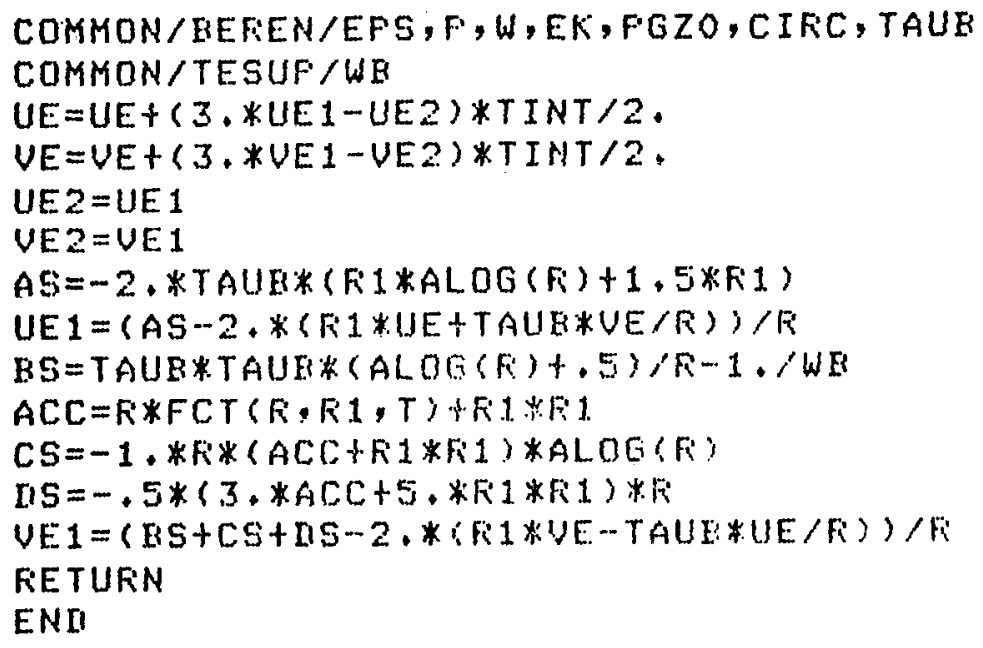


Tracor Hydronautics

SURROUTINE KUTTA(F,F1,H,FCT,T)
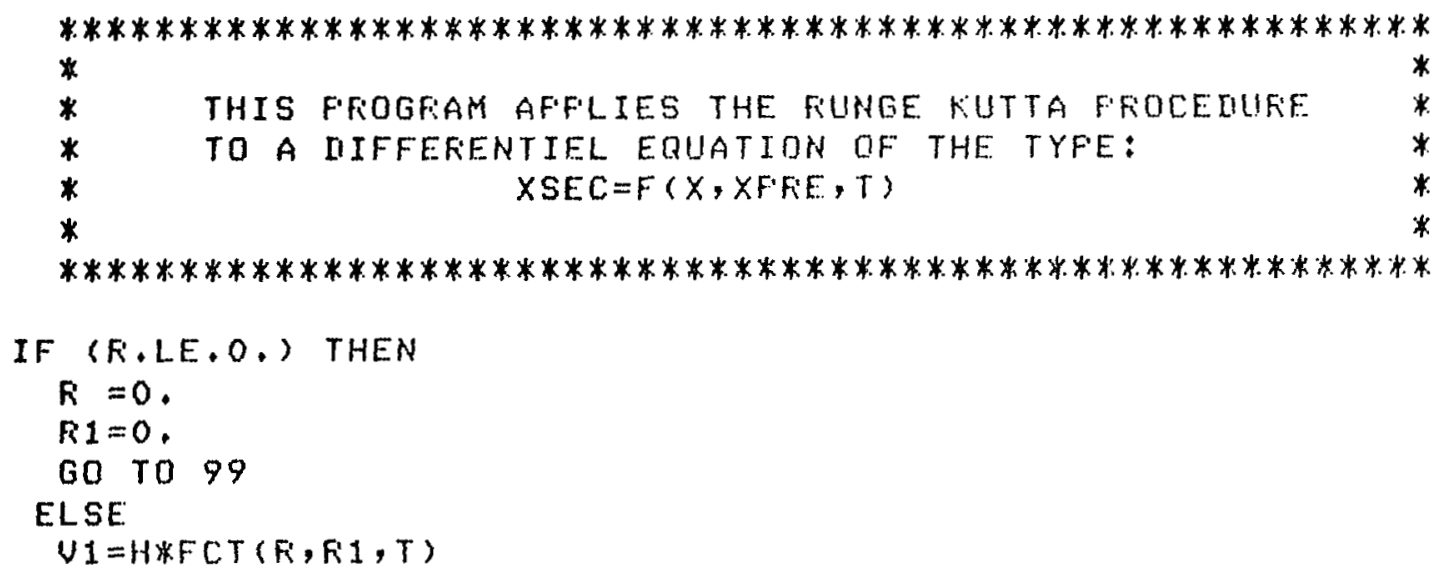

99 RETURN 
$-166-$

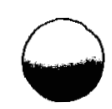

Jet Simulation Program 
FFOGFAM JETZN

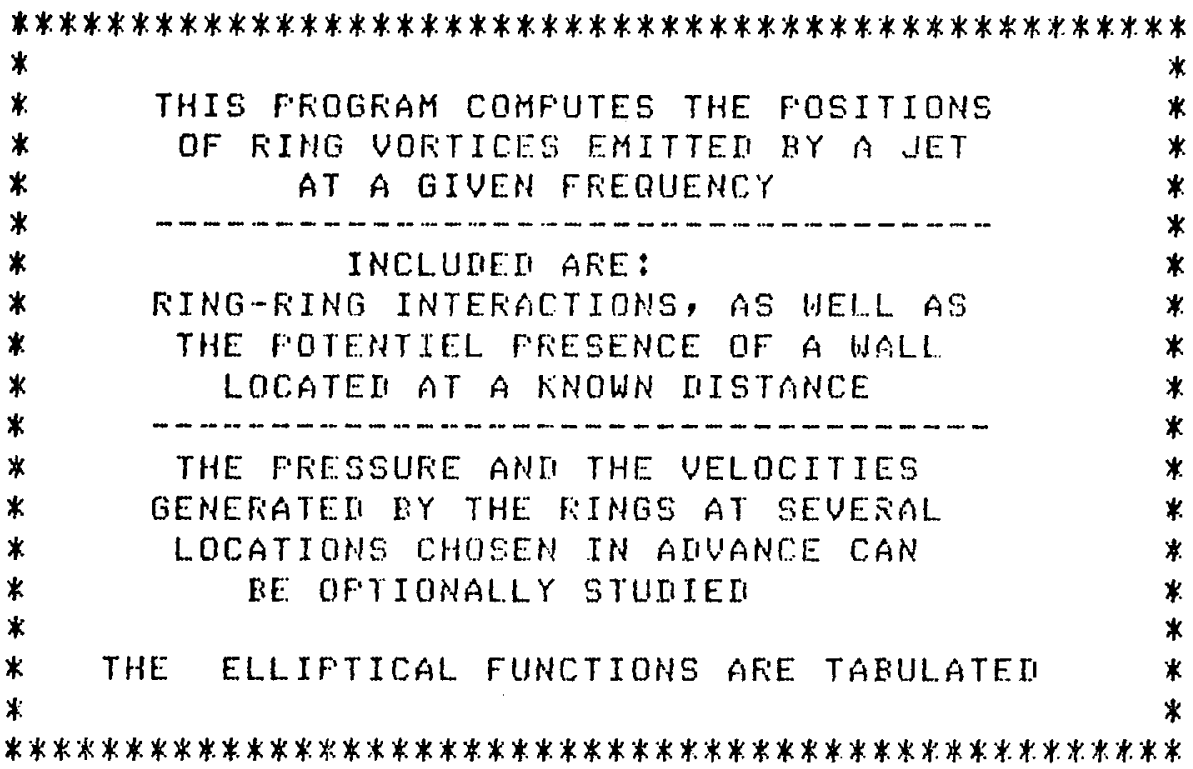

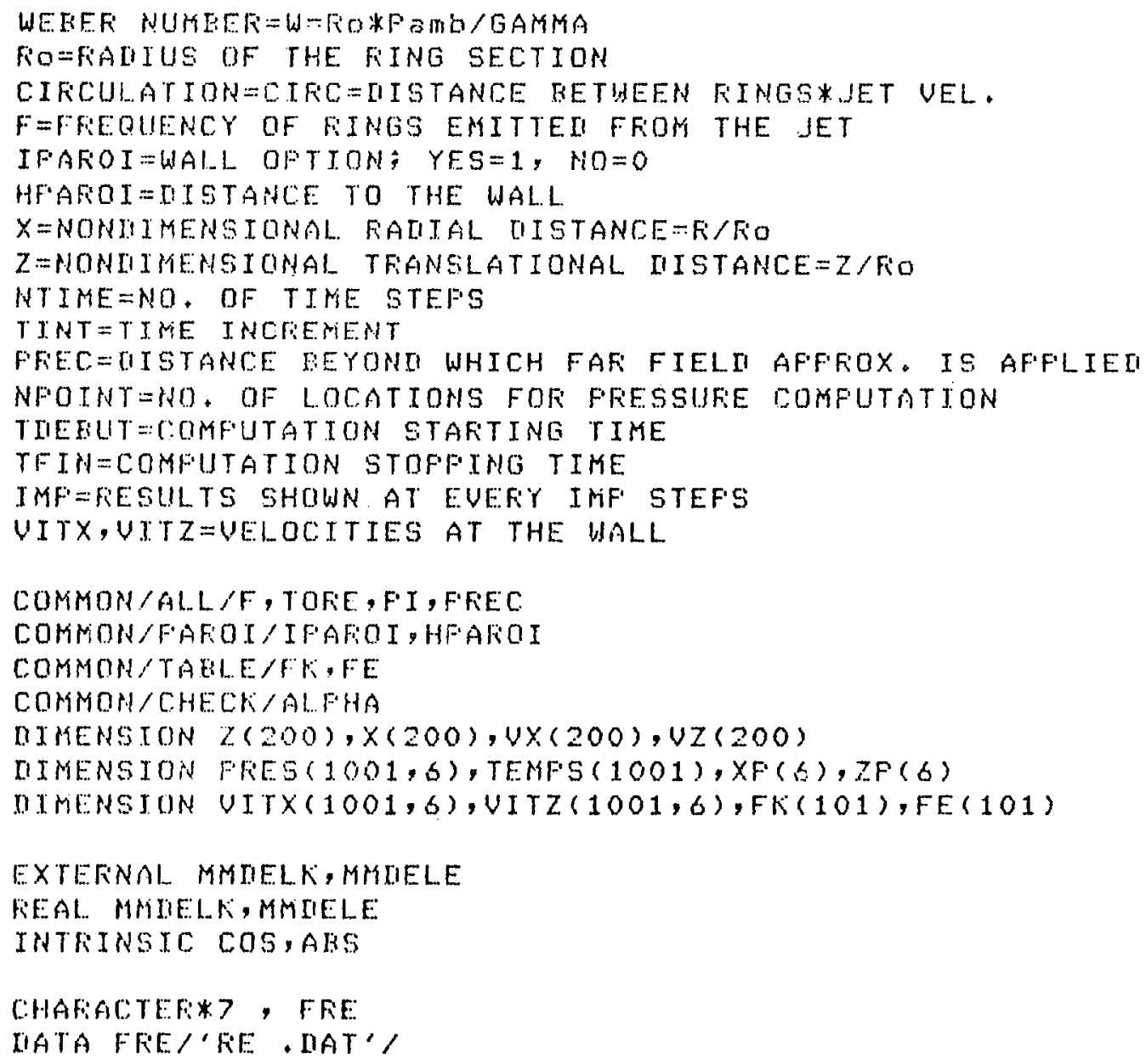




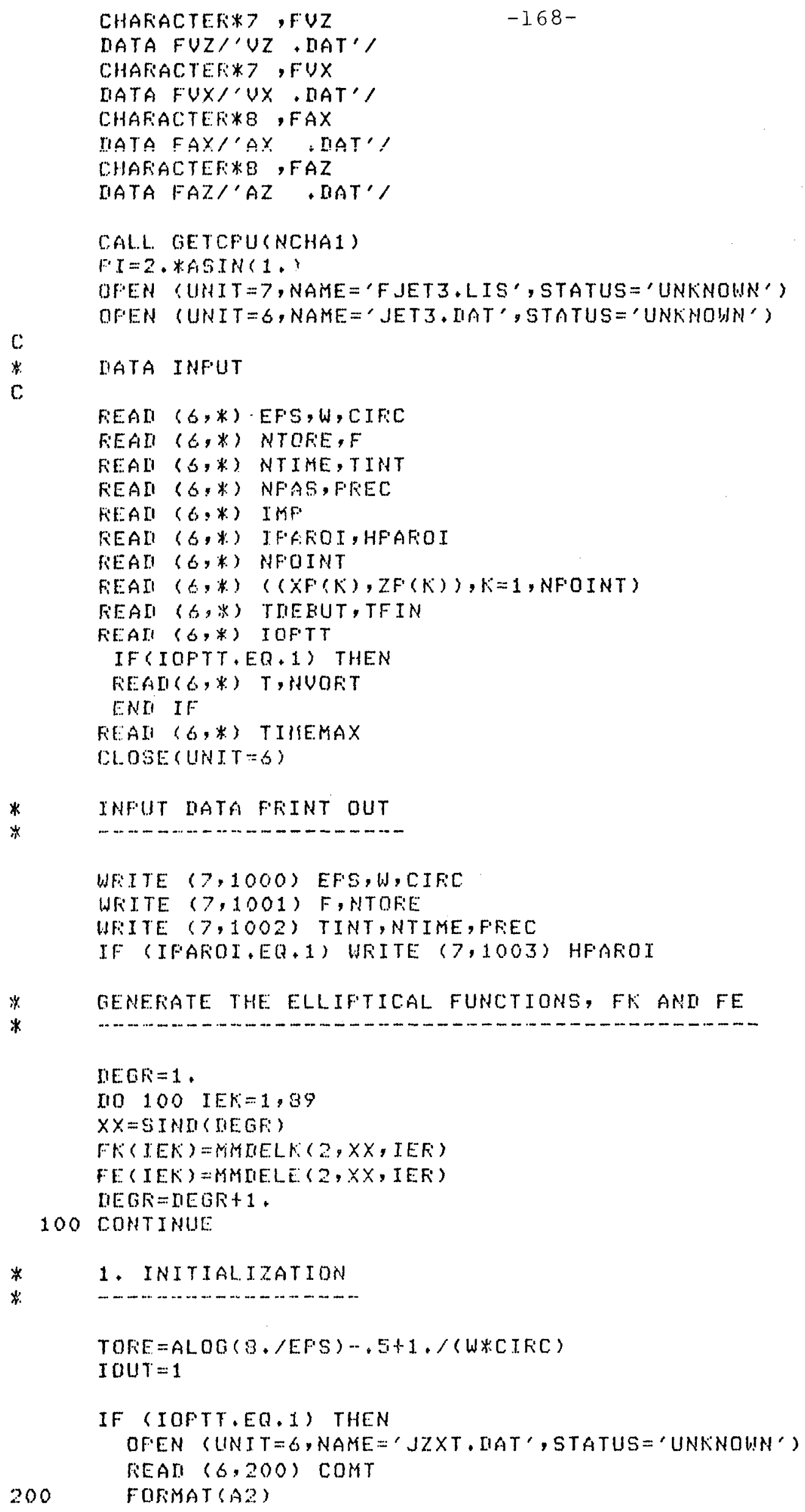




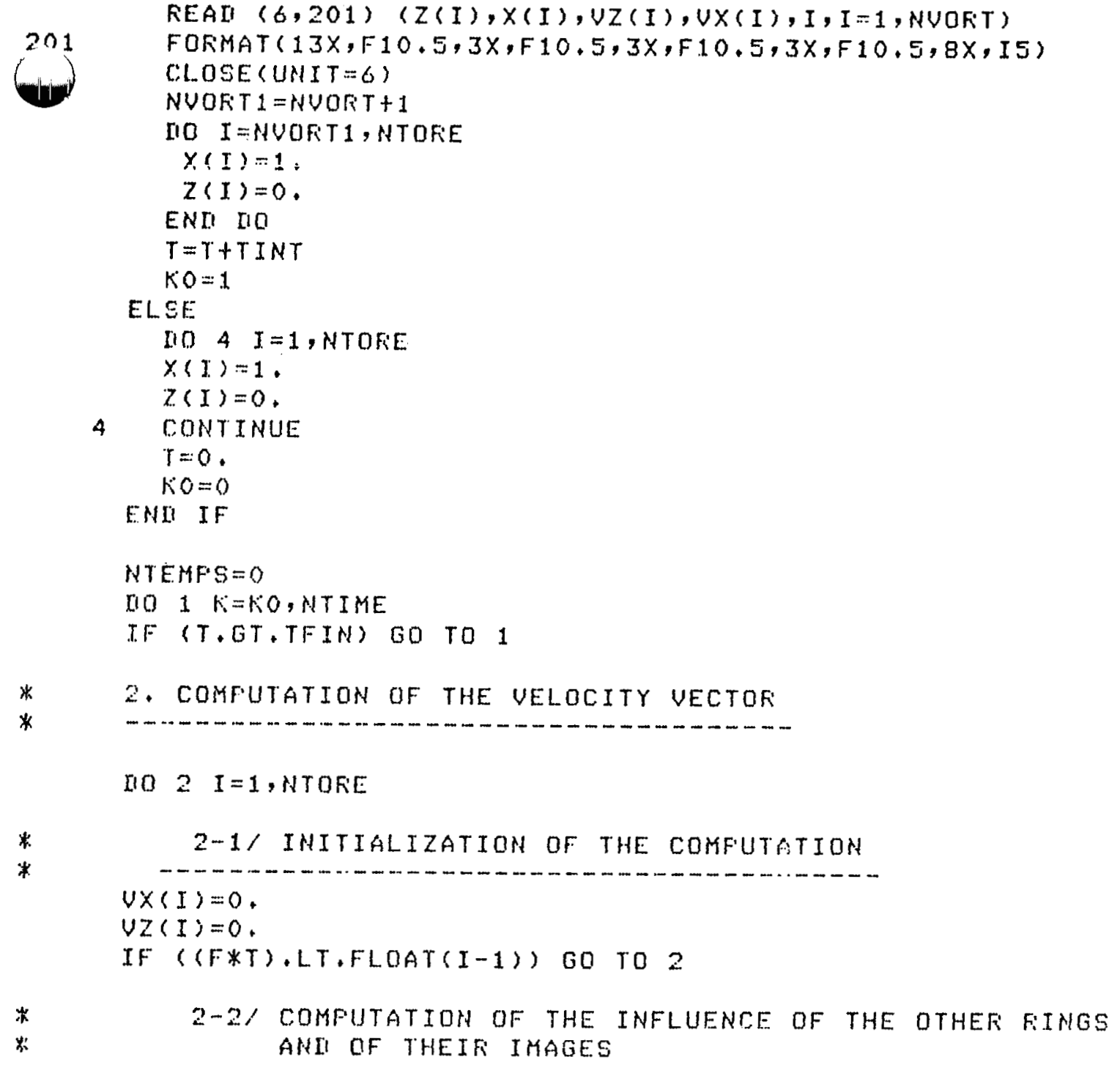
AHI OF THEIR IMAGES

IIO $3 J=1$, NTOKE

IF (J.EQ.I) GO TO 3

IF $\left\{F W^{*} T F L O A T(J-1)\right\rangle 3,5,5$

5 IF $((X(I), G T+20$.$) . OR. (X(J), G T \cdot 20)$.$) GO TO 22$

$U X(I)=U X(I)+U T N X(X(J), X(I), Z(I)-Z(J))$

$V Z(I)=V Z(I)+U I N Z(X(J): X(I), Z(I)-Z(J))$

60 TO 31

$22 \quad V \times(I)=0$.

$V Z(I)=0$.

31 IF (IFAFOI,EQ.0) GO TO 3

IF $((X(I), G T, 20),, 0 F+(X(J), G T, 20)$,$) GO TO 32$

$U X(I)=U X(I)+U I N X(X(J), X(I), 2$, *HFAFOI $-Z(I)-Z(J))$

$U Z(I)=U Z(I)-U I N Z\left(X(J), X(I), 2, * H F^{\prime} A F I I-Z(I)-Z(J)\right)$

60 TO 3

$32 \cup X(I)=0$.

$V Z(I)=0$.

3 CONTINUE

2-3/ COMFUTATION OF THE SELF INIUCEI VELOCITY

IF $((X(I), G T \cdot 20) .0 F,.(X(J), G T .20)$.$) GO TO 23$

$U Z(I)=U Z(I)+U \operatorname{IND} 1(X(I))$ 


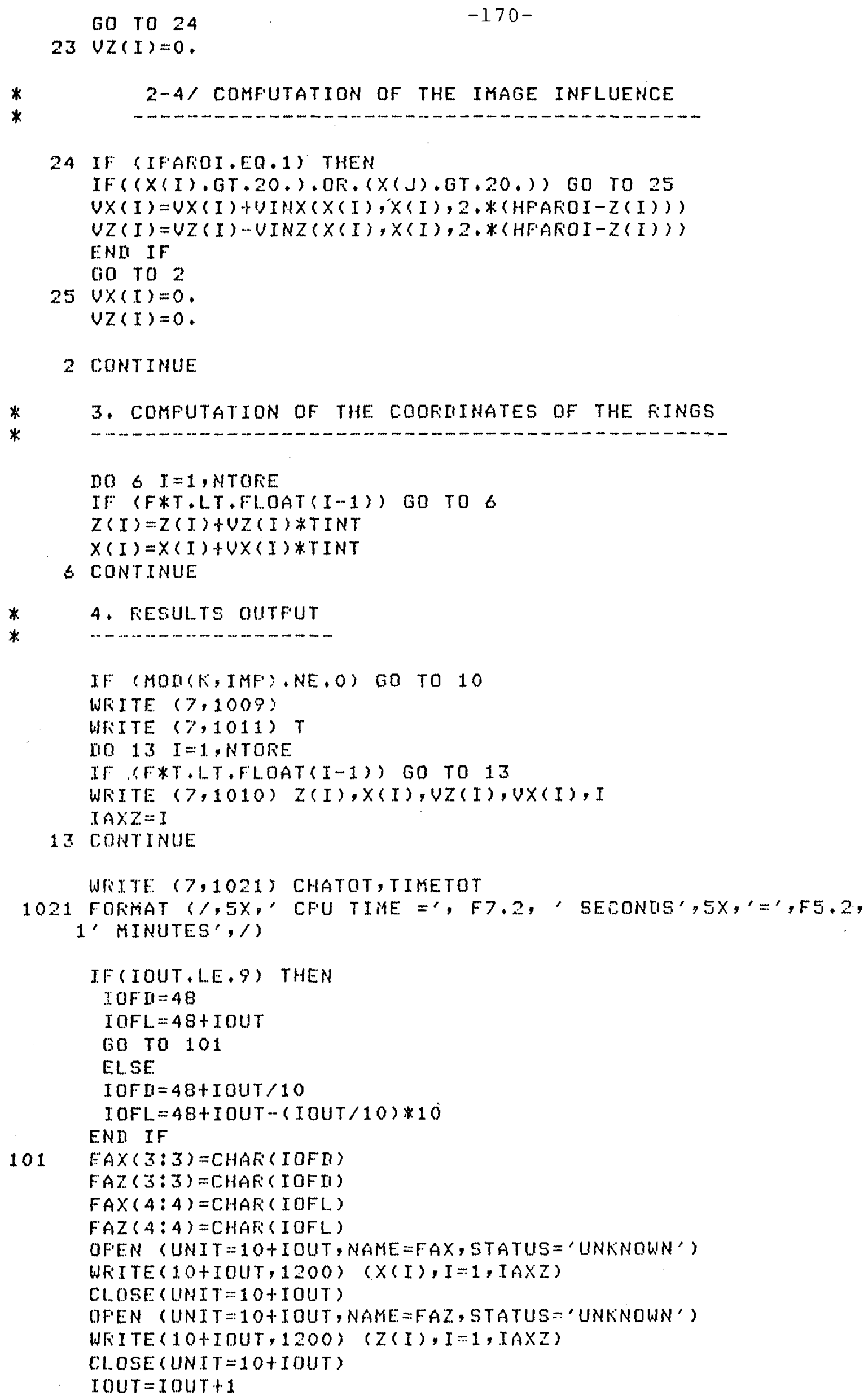


10 CONTINUE

IF (T.LT.TIEEUT) GO TO 18

IF $(M O I)(K, 20)$.NE. O) GO TO 18

* 5. pFesslife computation at fixen locations

*

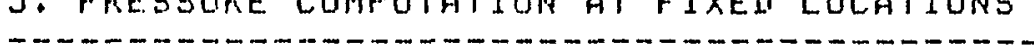

NTEMFS= NTEMFS +1

TEMFS (NTEMFS) $=T$

I. 14 IF: $=1$, NFOINT

5-1/ COMFUTATIOR OF [1-FHI / D-T

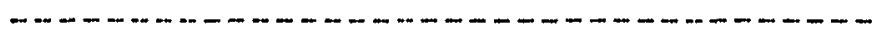

CALL FHIINST( $T, X, Z, U X, U Z, N T O R E, X F^{\circ}(I F), Z F$ (IF), IFHITOT)

5-2/ COMFUTATION OF GRAIR(FHI) $* 2$

CALL GRADFHI ( $T, X, Z, N T O F E, X F^{\prime}$ (IF), ZF(IF), UXFT, UZFT)

UITX (NTEMFS, IF) $=$ UXFT

UITZ(NTENFS, IF) =UZFT

FRES (NTEMFS, IF ) =IFHITOT-(UXFT**2, +UZFT**2,

14 CONTINUE

18 CONTINUE

$T=r+T r N r$

TF (MONULI, 1O),EQ.O) THEN

CALL GETCFU(NCHA2)

CHATOT $=$ (NCHA2-NCHA1)/100.

TIMETOT:- CHATOT/6O.

IF (TIMETOT , GT, TIMEMAX) GO TO 20

ENI IF

1 CONTINUE

20 CONTINUE

OFEN(URST T =10, NAME='TIME . IAT', STATUS='URKNOWN')

WRITE $(10,1200$ ) (TEMFS (I), I $=1$, NTEMPS)

CLOSE (UNIT $=10)$

DIO $7 I=1$, NF OINT

IF $L=I+48$

FliE: $(3: 3)=$ CHAF (IFL)

FUX $(3: 3)=$ CHAFi (IFL)

FUY $(3: 3)=$ CHAF $(I F L)$

OFEN(UINIT $=10+I$, NAME=FFE, STATUS='UNKNOWN')

WFI I TE $(10+T, 1200)$ (FFES(IT,I), IT=1,NTEMFS)

CL.OSE (UNIT $=10+\mathrm{I}$ )

OFEN(UNIT $=10+I$, NAME =FUX, STATUS= 'UNKNOWN')

WFITE(10+I, 1200) (UITX(IT,I), IT $=1$, NTEMFS)

CLOSE (UNIT $=10+I)$ 
OFEN(UNIT $=10+I$, NAME =FUZ, STATUS= 'UNKNOWN')

WRITE $(10+I, 1200)$ (UITZ(IT,I),IT=1,NTEMFS)

CLOSE (UNIT $=10+I)$

WRITE(7,1201) XF(I),ZF(I)

WFITE $(7,1.203)$

WFITE(7,1202) (TEMFS(IT),FFES(IT,I), VITX(IT,I), VITZ(IT,I),

1

$I T=1, N T E M F S$ )

7 CONTINUE

1000 FOFMAT $15 X$, , FING INTEFACTION',//, $5 X, '$ CHAFACTEFISTICS OF THE FI

INGS', $, 5 X,{ }^{\prime} E F S=, F 10.5, /, 5 X, ' W=,, F 10.5, /, 5 X, ' C I F C='$, $1 F 10.5)$

1001 FOFMAT $S X$, 'FFEQUENCY OF RIHGS EMISSIOH: $F=, F 10.5,1,5 X$,

1'NUMBER OF FIINGS: NTOFE $=$ 'I5,')

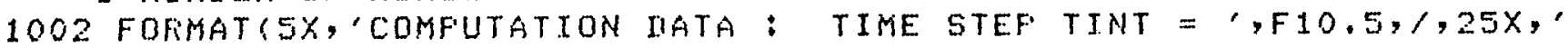

INUMHER OF TIME STEFS NTIME $=$, IS, $/ 25 X$, IIISTANCE FOF THE FAF

1FIELII AFFFIXXIMATION $=$,F10,5, 1 )

1003 FORMAT $25 X$, "IISTANCE FEOM THE WALL, Z/FIO = ,F $10.5,1)$

1009 FOFMATU/,' INSTANT $T$ COOFII. $Z$ COOFII, $X$ SFEEI $Z$

IFEEI $X$ FING NO',/)

1010 FORMAT $(13 X, F 10,5,3 X, F 10,5,3 X, F 10,5,3 X, F 10.5,8 X, 15)$

1011. FORMAT $(1,1 \times, F 10,5)$

1200 FOFMAT (5E15.6,1X,'\&')

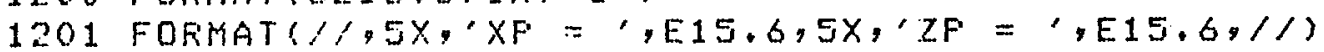

1202 FOFIMAT $(5 X, 4 E 15,6)$

1203 FOFMAT $11 X, ' T$ ', 10X,'FFES',11X,'UITX',11X,'UITZ', $)$

WFITE $(7,1020)$ CHATOT, TIMETOT

1020 FOFMAT (',', TOTAL CFU TIME $=\prime, F 7.2, '$ SECOMIS',5X,'=',F5.2, 1' MINUTES')

STOF

END 
DISTRYBUTION:

TII-4500-R66-UC-66C (507)

Anoco Production Company

Research Center

P.0. Box 591

Tulsa, Oklahoma 74102

Attn: Tom Warren

Department of Energy

Albuquerque Operations office

Albuquerque, NM 87185

Attn: Larry Diamond

Halliburton

Drawer 1431

Duncan, Oklahoma 73533

fiten: Dwight Smith

B. J. Livesay

129 Liverpoo1

Cardiff, California 92007

Los Alamos National Laboratory

Mail Stop 570

Los Alamos, New Mexico 87545

Attn: John C. Rowley

Harvey E. Mallory

P.0. Box 54696

Tulsa, Oklahoma 74155

NL Baroid Petroleum Services

City Centre Building, Suite $365 \mathrm{~W}$

6400 Uptown Boulevard, N.E.

Albuquerque, NM 87110

Attn: Gene Polk

NL Petroleum Services

P.O. Box 60087

Houston, Texas 77205

Attn: John E. Fontenot

Otis

Research and Development

P.0. Box 34380

Da1las, Texas 75234

Attn: William D. Rumbaugh

Phillips Petroleum Company

Geothermal Operations

655 East 4500 South

Salt Lake City, Utah 84107

Attn: Ton Turner

She11 Oil Company

Two Shell Plaza

P.0. Box 2099

Houston, Texas 77001

Attn: Ed Bingman

Superior 0i1

Eastern Bivision

P.0. Box 51108 OCS

Lafayette, Louisiana 70505

Attn: Ed Martin

Texas A\&M University

College Station, Texas 77843

Attn: Professor Melvin Friedman

Dept. of Geology

Tracor Hydronautics

7210 Pinde11 School Rd.

Laure1, MD 20707

Attn: Georges Chahine

Union Geothermal Division

Union Oil Company of California

Union Oil Center

Los Angeles, Caifornia 90017

Attn: De1 E. Pyle

U. S. Department of Energy (4)

Geothermal Hydropower

Technologies Division

Forrestal BIdg., CE 324

1000 Independence Ave., S.W.

Washington, DC 20585

Attn: J. Bresee

J. Rannels

R. Toms

D. Allen

Drilling Fluid Consultants 17726 S.W. Overlook Ln.

Lake Osewego, Oregon 97034

Attn: Tom Anderson 
DISTRIBITION (Continued)

$\begin{array}{ll}3141 & \text { L. J. Erickson (5) } \\ 3151 & \text { W. L. Garner (3) } \\ 6000 & \text { E. H. Beckner } \\ 6200 & \text { V. L. Dugan } \\ 6240 & \text { R. K. Traeger } \\ 6241 & \text { J. R. Kelsey (40) } \\ 6241 & \text { D. A. Glowka (10) } \\ 6246 & \text { B. Granoff } \\ 6247 & \text { P. J. Honmert } \\ 6250 & \text { B. W. Marsha11 } \\ 8214 & \text { M. A. Pound }\end{array}$

מT U.S. GOVERNMENT PRINTING OFFICE:1984-576-035 / 4392 